\title{
Catalysis-Enabled Access to Cryptic Geldanamycin Oxides
}

Margaret J. Hilton, Christopher Brackett, Brandon Q. Mercado, Brian S. J. Blagg, Scott Miller

Submitted date: 08/01/2020 Posted date: 09/01/2020

Licence: CC BY-NC-ND 4.0

Citation information: Hilton, Margaret J.; Brackett, Christopher; Mercado, Brandon Q.; Blagg, Brian S. J.;

Miller, Scott (2020): Catalysis-Enabled Access to Cryptic Geldanamycin Oxides. ChemRxiv. Preprint. https://doi.org/10.26434/chemrxiv.11550714.v1

Catalytic, selective modifications of natural products can be a fertile platform for unveiling not only new natural product analogs with altered biological activity, but also for revealing new reactivity and selectivity hierarchies for embedded functional groups in complex environments. Motivated by these intersecting aims, we report site and stereoselective oxidation reactions of geldanamycin facilitated by aspartyl-peptide catalysts. Through the isolation and characterization of four new geldanamycin oxides, we discovered a synergistic effect between lead peptide-based catalysts and geldanamycin, resulting in an unexpected reaction pathway. Curiously, it seems unlikely that our discoveries would not have been possible absent the outer sphere interactions intrinsic to both the catalyst and the natural product. The result is a set of new "meta" catalytic reactions that deliver both unknown and previously incompletely characterized geldanamycin analogs. Enabled by the catalytic, site-selective epoxidation of geldanamycin, biological assays were carried out to document the bioactivities of the new compounds.

File list (2)

(b)-Manuscript.pdf (1.08 MiB) view on ChemRxiv - download file

(c)-SI.pdf (2.66 MiB) view on ChemRxiv d download file 


\title{
Catalysis-Enabled Access to Cryptic Geldanamycin Oxides
}

\author{
Margaret J. Hilton, ${ }^{\dagger}$ Christopher Brackett, ${ }^{\dagger}$ Brandon Q. Mercado, ${ }^{\dagger}$ Brian S. J. Blagg, ${ }^{\ddagger}$ Scott J. Miller ${ }^{\dagger *}$ \\ 'Department of Chemistry, Yale University, New Haven, Connecticut 06520, United States \\ Department of Chemistry and Biochemistry, University of Notre Dame, Notre Dame, Indiana 46556, United States \\ Supporting Information Placeholder
}

\begin{abstract}
analogs with altered biological activity, but also for revealing new reactivity and selectivity hierarchies for embedded functional groups in complex environments. Motivated by these intersecting aims, we report site and stereoselective oxidation reactions of geldanamycin facilitated by aspartyl-peptide catalysts. Through the isolation and characterization of four new geldanamycin oxides, we discovered a synergistic effect between lead peptide-based catalysts and geldanamycin, resulting in an unexpected reaction pathway. Curiously, it seems unlikely that our discoveries would not have been possible absent the outer sphere interactions intrinsic to both the catalyst and the natural product. The result is a set of new "meta" catalytic reactions that deliver both unknown and previously incompletely characterized geldanamycin analogs. Enabled by the catalytic, site-selective epoxidation of geldanamycin, biological assays were carried out to document the bioactivities of the new compounds.
\end{abstract}

ABSTRACT: Catalytic, selective modifications of natural products can be a fertile platform for unveiling not only new natural product

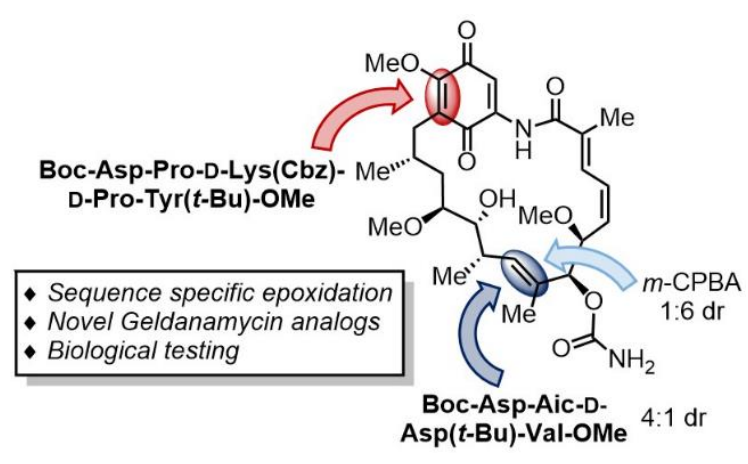

\section{INTRODUCTION}

Catalysis in chemistry and biochemistry is founded upon accelerating reaction rates and achieving control over some selectivity issue. ${ }^{1,2}$ Accordingly, the field of "asymmetric catalysis" continues to blossom as an ever more powerful way of producing stereochemically homogeneous building blocks with high efficiency. ${ }^{3}$ Directing chiral catalysts towards complex substrates, such as bioactive natural products, creates a further challenge in addition to enantioselectivity, involving the differentiation of functional groups within the same compound. ${ }^{4,5}$ If more than one copy of the same reactive functional group exists in the structure, then the issue of stereoselectivity is compounded by that of site selectivity, resulting in a demanding landscape of partitioned pathways that lead to many different products to traverse. As a pragmatic solution to these multifaceted selectivity challenges, the application of a diverse catalyst library to a given scaffold offers the opportunity to simultaneously assess (a) multiproduct reaction outcomes and (b) to achieve analogs that might not be available in a straightforward manner by either biosynthetic or chemical methods. In addition, and perhaps most alluringly, subjecting complex molecules to catalyst libraries also creates the opportunity to unveil unexpected reactivity, leading to compounds that might not be targeted at all based on canonical reactivity patterns. This paper discloses findings along all of these lines, wherein we have observed an unanticipated cooperativity between a complex substrate of interest and various catalysts. Accordingly, our study has provided a number of perhaps "cryptic" natural product analogs.

In terms of project design, we were curious about expanding the breadth of aspartic acid-based peptides in the context of natural product derivatization. In the field of asymmetric catalysis, for small molecule functionalization, we had previously demonstrated that Asp-containing peptides are efficient and selective catalysts for
Figure 1. Overview of Aspartyl-Peptide Catalyzed Reactions and Geldanamycin
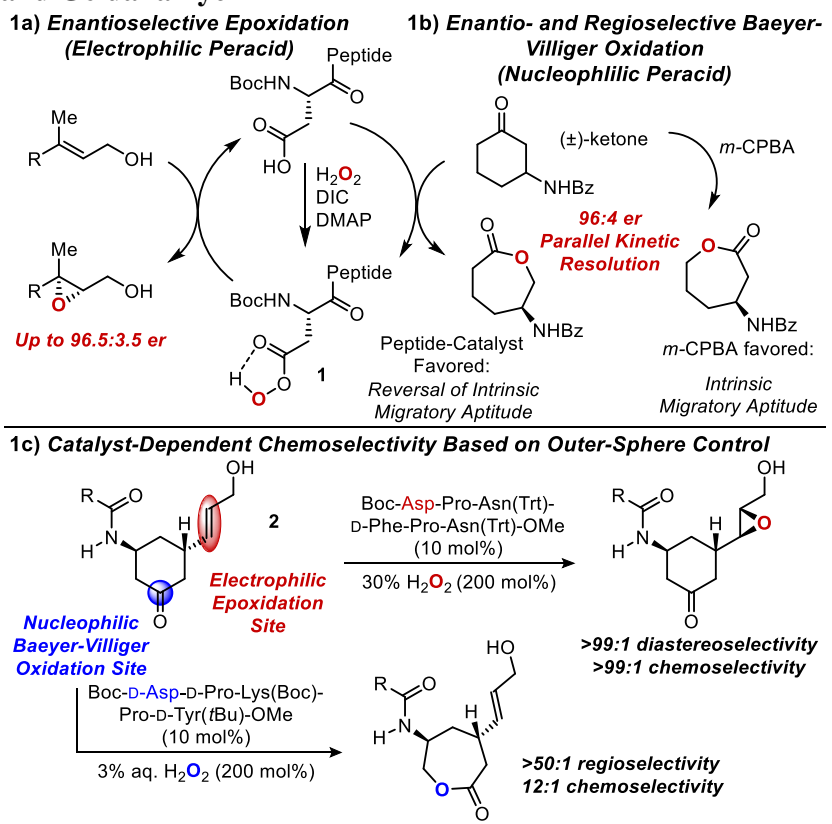

1d) Overview of Geldanamycin
- ansamycin family
promising anti-cancer activity
inhibits Hsp90
poor bioavailability, hepatotoxic
- soluble in organic solvents 
both enantioselective alkene epoxidation (Figure 1a), ${ }^{6,7}$ as well as Baeyer Villiger oxidation ${ }^{8,9}$ with then-unprecedented catalystcontrolled reversals of intrinsic migratory aptitude tendencies (Figure 1b). Mechanistically, the key has been a catalytic shuttle between the aspartic acid catalyst, and its reactive aspartyl peracid form (1), which transfers the $\mathrm{O}$-atom in each scenario. We had also shown that a predictive choice of catalyst (i.e., peptide-sequenceselected) could be employed to predetermine whether epoxidation or Baeyer-Villiger oxidation would occur, at least with a carefully designed, admittedly "rigged" substrate 2 (Figure 1c). ${ }^{9}$ Accordingly, a critical next step for the advancement of catalystdependent, site-selective diversification of complex natural products with the Asp-based catalyst paradigm is to demonstrate feasibility with genuinely complex bioactive structures. For this purpose, we chose to explore the catalyst-dependent diversification of geldanamycin using Asp-containing peptides (Figure 1d).

Geldanamycin exhibits profound biological activity, targeting Hsp90, ${ }^{10,11}$ a molecular chaperone responsible for folding, stabilization, and maturation of many client proteins, and has shown promise as an anti-cancer therapeutic. ${ }^{12-15}$ However, geldanamycin exhibits poor bioavailability and hepatotoxicity, ${ }^{16}$ stimulating the pursuit of new analogues to address these issues via total $^{17,18}$ and semi-synthetic methods. ${ }^{19-22}$ In fact, geldanamycin derivatives, such as 17-allylamino-17-demethoxygeldanamycin (17-AAG),${ }^{23}$ have reached clinical trials for the treatment of many types of cancer, including colorectal, breast, ovarian, lung, multiple myeloma, and leukemia. ${ }^{24}$ In addition, geldanamycin is a challenge for confronting chemoselectivity, as it contains two alkenyl regions for epoxidation - a diene and an isolated trisubstituted alkene, along with quinoid functionality replete with unsaturation. There- fore, multiple alkene sites may be subject to functionalization, along with the consideration of $\pi$-facial selectivity that could lead to various diastereomers from the epoxidation of each alkene site. Finally, practical considerations were also favorable, such as accessibility to the compound and solubility in a variety of reaction-compatible solvents.

\section{RESULTS AND DISCUSSION}

Our survey of aspartyl peptide-based catalysts produced interesting and unexpected results immediately. As is discussed in detail below, four principle mono-oxide products of geldanamycin are observed in these reactions, and their ratios may be extensively modulated with catalyst-dependent kinetic control. Two of the compounds resolve long-standing ambiguities regarding diastereomerism of the geldanamycin-derived epoxides, ${ }^{14,21}$ one of which is efficiently accessed for the first time based on a unique, peptide-based catalyst. Two other compounds, each also unveiled for the first time as a result of a specific aspartyl peptide-based catalyst, are the products of an unanticipated reaction pathway. Moreover, observation of these compounds emerges from an unanticipated cooperation between the complexity of the peptidebased chiral catalysts and the embedded functional group arrays within the macrocyclic molecular scaffold of geldanamycin. This symbiosis, between complex chiral catalysts and complex substrates, enables explicit biological testing of these new and fully characterized compounds, several of which represent structures that were outside of our original conception, grounded in either rational design or traditional scaffold diversification analysis.

Chemistry. Initiating our investigations, geldanamycin oxidation was tested using $m$-CPBA to establish inherent reactivity

Figure 2. Initial Peptide Evaluation ${ }^{a, b}$ and Structure Determination

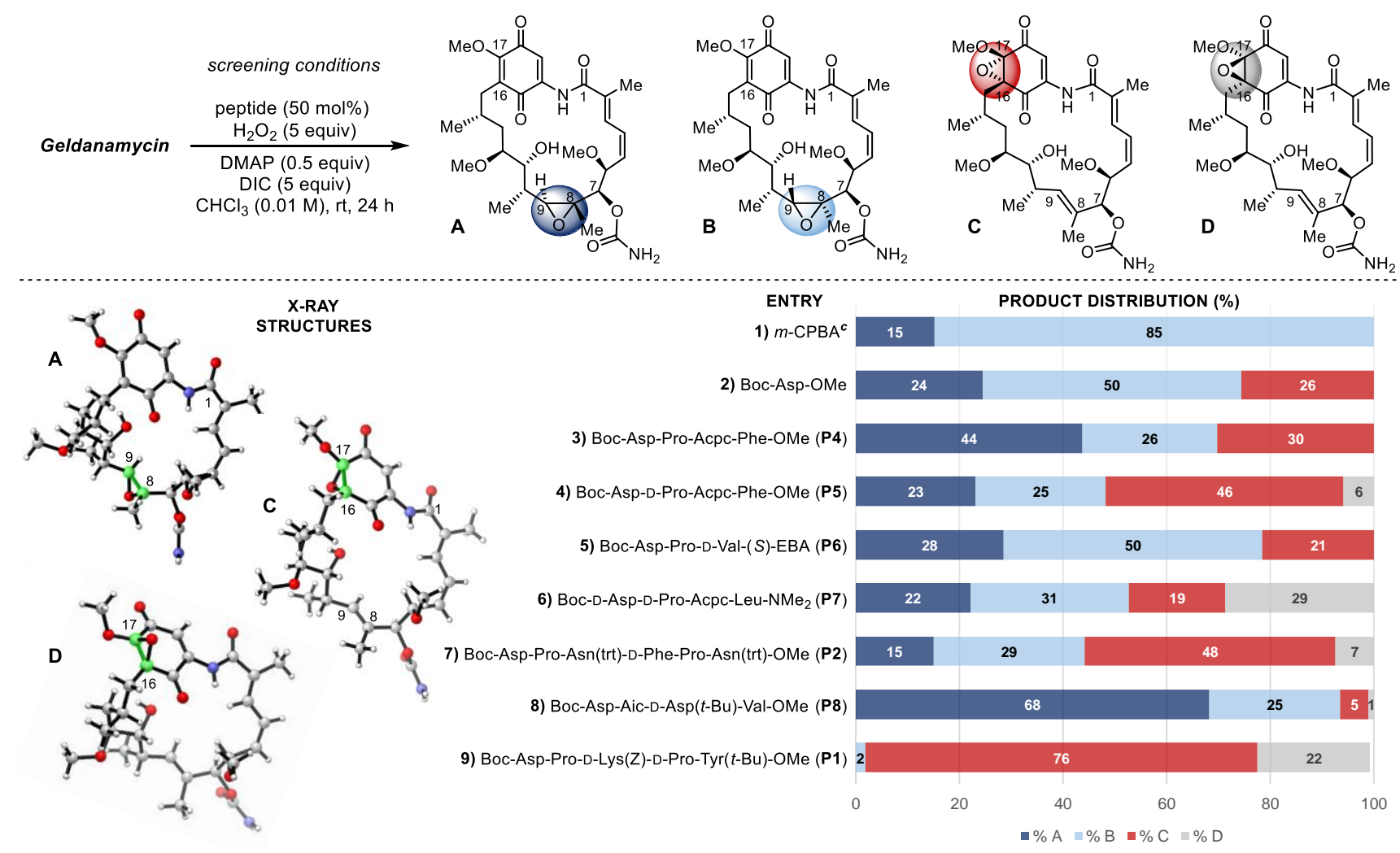

${ }^{a}$ Standard conditions: Geldanamycin $(2.8 \mathrm{mg}, 5 \mu \mathrm{mol}, 1$ equiv), peptide $(50 \mathrm{~mol} \%)$, 4-dimethylaminopyridine (DMAP, $50 \mathrm{~mol} \%)$, aqueous $\mathrm{H}_{2} \mathrm{O}_{2}(30 \mathrm{wt} \%$; 5.0 equiv), $\mathrm{N}, \mathrm{N}^{\prime}$-diisopropylcarbodiimide (DIC, 5.0 equiv), $\mathrm{CHCl}_{3}(0.01 \mathrm{M}), 22{ }^{\circ} \mathrm{C}, 24 \mathrm{~h}$. ${ }^{\mathrm{b}}$ Average from two trials. Determined using UPLC from crude reaction mixtures with an internal standard and calibration curve. Product distributions are corrected to total $100 \%$. See SI for yields. ${ }^{\circ}$ Conditions: Geldanamycin $\left(2.8 \mathrm{mg}, 5 \mu \mathrm{mol}, 1\right.$ equiv), $m$-chloroperoxybenzoic acid $\left(m\right.$-CPBA, 2 equiv), $\mathrm{CHCl}_{3}(0.01 \mathrm{M}), 22^{\circ} \mathrm{C}, 24 \mathrm{~h}$. (Abbreviations: Boc $=$ tertbutyloxycarbonyl; Aic = 2-aminoindane carboxyl; $\mathrm{Z}=\mathrm{Cbz}=$ carboxybenzyl; Acpc $=1$-aminocyclopropane carboxyl; EBA = ethylbenzylamine; trt = trityl) 
and selectivity, as these results are likely to be minimally biased by outer sphere noncovalent interactions between the reactive species and substrate, beyond the traditional Henbest paradigm. ${ }^{25}$ Reaction of geldanamycin with $m$-CPBA cleanly yielded two mono-oxidized products in a 15:85 ratio (A:B, Figure 2, entry 1), strongly favoring compound $\mathbf{B}$. Upon isolation and characterization by NMR spectroscopy, it was determined that $\mathbf{A}$ and $\mathbf{B}$ are diastereomeric epoxides, derived from reaction of the 8,9-trisubstituted alkene. These compounds have been reported previously, albeit in the absence of either a relative or absolute stereochemical assignment. ${ }^{14,21}$ Nor had either been produced with catalystdependent selectivity, to our knowledge. In our hands, we were delighted to achieve X-ray quality crystals of A. Analysis of the $\mathrm{X}$-ray crystal structure revealed the complete stereochemistry of the compound, displaying that epoxidation occurs from the "bottom" face, as drawn, to yield A, implying that the $m$-CPBAfavored product $\mathbf{B}$ is the result of epoxidation of the "top" alkene face.

The coin of the realm in studies of site-selective catalysis is the catalyst-dependent reversal of the intrinsic selectivity patterns observed with simple reagents and catalysts. Thus, we then transitioned to the exploration of Asp-based catalysts. Rather surprisingly, results divergent from those observed with $m$-CPBA surfaced immediately. Using the monomeric Boc-Asp-OMe, the $m$ CPBA-favored product $\mathbf{B}$ is still favored over $\mathbf{A}$, although to a lesser degree, and a new product $(\mathbf{C})$ is now observed (Figure 2, entry 2). Interestingly, $\mathbf{C}$ results from epoxidation of the quinone at the 16,17-position, a result also confirmed by X-ray crystallography analysis. Further evaluation of catalysts (vide infra) led to the observation of product $\mathbf{D}$, which also results from quinone epoxidation at the 16,17-position, yet from the alternate $\pi$ face in comparison to $\mathbf{C}$. This structure was also established by $\mathrm{X}$ ray crystallography analysis. In general, other minor products, such as from bis-oxidation or diene epoxidation, may be produced, yet low yields $(<5 \%)$ excluded these from our analysis. These observations alone struck us as quite noteworthy. The most preliminary survey of Asp-based catalysts rapidly delivered three alternative products relative to the major compound produced by $m$-CPBA. Two of these geldanamycin oxides resolved longstanding structural ambiguity $(\mathbf{A}$ and $\mathbf{B})$, and two $(\mathbf{C}$ and $\mathbf{D})$ proved to be new geldanamycin scaffolds, unknown prior to the present studies, to our knowledge. Overall, all four compounds provided new insight into reactivity trends of both geldanamycin and Aspcontaining peptide catalysts.

To assess the extent to which these catalyst-dependent product ratios could be varied, a library of 14 Asp-containing peptides was selected from previous projects and was then evaluated (Figure 2, select results shown; for full list, see SI). Low selectivities were measured for predictable $\beta$-turn-biased and/or $\beta$-hairpin-biased sequences $^{26-28}$ (entries 3-4), including two peptides with C-terminal amide caps (entries 5-6). A legacy catalyst for allylic alkene epoxidation ${ }^{7}$ slightly favors product $\mathbf{C}$ (entry $7, \mathbf{P 2}$ ). Both reactivity and selectivity for 8,9-epoxidation improved significantly when Boc-Asp-Aic-D-Asp(t-Bu)-Val-OMe (P8) was employed (entry 8). The formation of products $\mathbf{C}$ and $\mathbf{D}$ is largely suppressed with this more reactive peptide, resulting in a distribution of $68 \%, 25 \%, 5 \%$, and $1 \%$, respectively $(\mathbf{A}, \mathbf{B}, \mathbf{C}$, and $\mathbf{D}$, entry 8$)$. In contrast to the reaction using $m$-CPBA, which yielded $\mathbf{A}$ and $\mathbf{B}$ in a 1:5.7 dr, $\mathbf{P 8}$ provides a coveted reversal of diastereoselectivity, delivering a 2.7:1 dr (A:B). Finally, legacy catalyst P1, which was previously reported to promote Baeyer Villiger oxidation (Figure 1b), ${ }^{8}$ provides the most striking catalyst-dependent diversion from conventional reactivity, exhibiting 98\% selectivity for quinone epoxidation, affording products $\mathbf{C}$ and $\mathbf{D}$ in a 3.5:1 diastereomeric ratio (entry 9).

Thus, our initial examination of Asp-based peptides (Figure 2), yielded two catalysts that favor two distinct products, alternative to that favored by $m$-CPBA: P8, favoring 8,9-epoxide A; and P1, favoring 16,17-epoxide C. Moreover, the screening provides catalyst-dependent access to the unique epoxyquinones $\mathbf{C}$ and $\mathbf{D}$, which are not detected to an appreciable extent with $m$-CPBA. As these catalysts provide stark contrasts to the outcomes induced by $m$-CPBA, we wished to understand further the basis of these catalyst-controlled outcomes in hopes of some degree of further optimization.

To this end, several new catalysts were explored in the context of attempted optimization for product A (Figure 3). Relative to catalyst P8, exchanging Aic at the $i+1$ position for Acpc, D-Phe, or $\mathrm{D}-\mathrm{Val}$ resulted in similar product distributions (entries 1-3, respectively). However, $\mathbf{P 1 0}$ with Acpc in the $i+1$ position does provide a modest enhancement of $\mathbf{A}: \mathbf{B}$ ratio (3.1:1 dr), which could be further optimized (vide infra). On the other hand, if the $i+1$ residue is converted to an L-amino acid (Phe, Val, or Pro), the selectivity for A consecutively diminishes (entries 4-6, respectively). For example, with P15 (Pro at $i+1$ ), almost equal amounts of $\mathbf{A}, \mathbf{B}$, and $\mathbf{C}$ are observed (entry 6), as is seem with other peptides containing the sequence Asp-Pro (P2, P4, P6, Figure 2).

Figure 3. Point mutations of lead catalyst, A-Selective Catalyst, P10 ${ }^{a, b}$

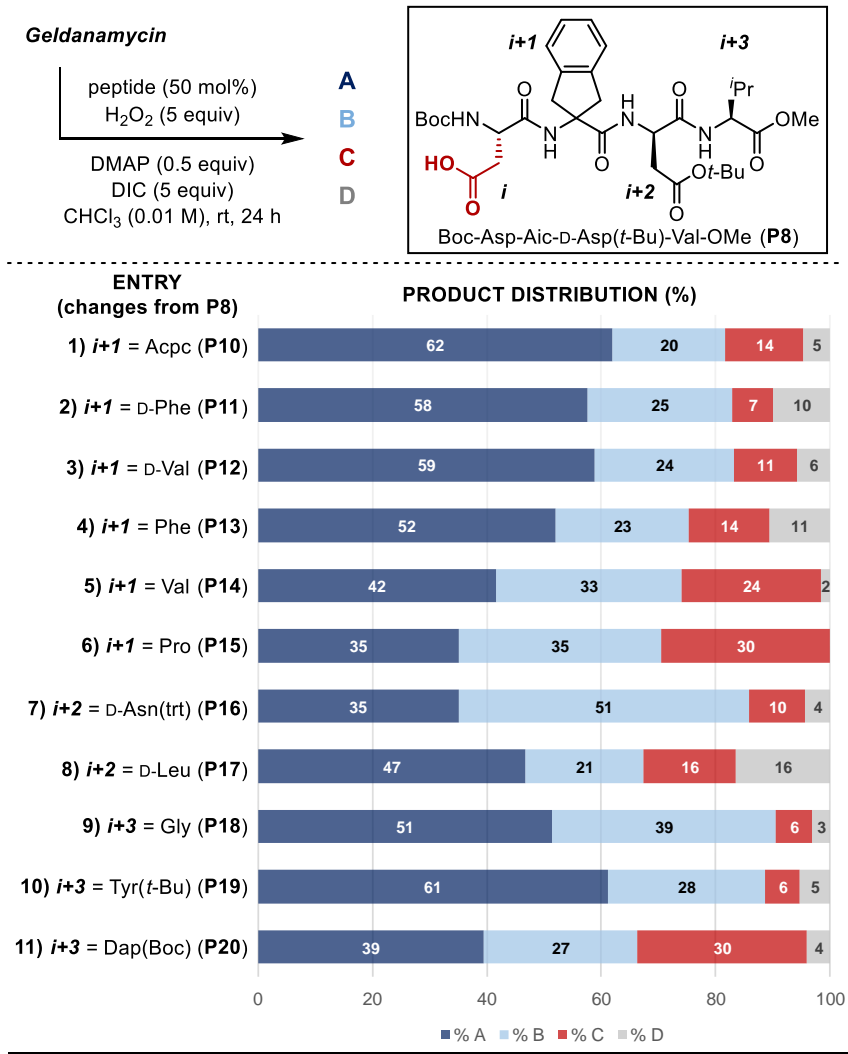

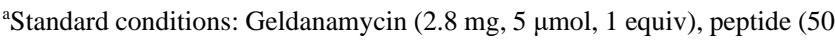
mol \%), 4-dimethylaminopyridine (DMAP, 50 mol \%), aqueous $\mathrm{H}_{2} \mathrm{O}_{2}(30$ wt \%; 5.0 equiv), $N, N^{\prime}$-diisopropylcarbodiimide (DIC, 5.0 equiv), $\mathrm{CHCl}_{3}$ $(0.01 \mathrm{M}), 22{ }^{\circ} \mathrm{C}, 24 \mathrm{~h} .{ }^{\mathrm{b}}$ Average from two trials. Determined using UPLC from crude reaction mixtures with an internal standard and calibration curve. Product distributions are corrected to total $100 \%$. (Abbreviations: Boc $=$ tert-butyloxycarbonyl; Acpc $=1$-aminocyclopropane carboxyl; Dap $=\mathrm{L}-2,3$-diaminopropionic acid)

As shown in Figure 3, additional changes at the $i+2$ position provided generally decreases in the selectivity for $\mathbf{A}$. When D$\mathrm{Asp}(t-\mathrm{Bu})$ is replaced with $\mathrm{D}$-Asn(trt) at $i+2$, thus adding a hydrogen-bond donor in place of an acceptor, selectivity for $\mathbf{A}$ decreases (entry 7). Intriguingly, when the possibility for 
hydrogen-bonding at this position is removed through insertion of a D-Leu residue, selectivity for $\mathbf{A}$ is restored to a small degree (entry 8). Finally, low steric bulk at $i+3$ (Gly, entry 9) causes diminished selectivity for product $\mathbf{A}$, yet a polar aromatic group ( $\operatorname{Tyr}(t-\mathrm{Bu})$, entry 10) did not significantly modulate the product distributions in comparison to $\mathbf{P 1 0}$. However, including a hydrogen-bond donor (Dap(Boc)) at $i+3$ produces a lower amount of $\mathbf{A}$, and a greater amount of $\mathbf{C}$ now emerges, affording almost equal yields of $\mathbf{A}, \mathbf{B}$, and $\mathbf{C}$ (entry 11). Overall, this series of peptide mutations revealed that most sequences, except those with additional hydrogen-bond donors, are likely accessing similar transition state ensembles, resulting in comparable energetic landscapes for the production of the major product, $\mathbf{A}$.

To gain additional structural information concerning both Aselective catalyst $\mathbf{P 1 0}$ and $\mathbf{C}$-selective P1, peptide truncation studies were performed. When A-selective catalyst $\mathbf{P 1 0}$ is shortened to the trimer Boc-Asp-Acpc-D-Asp(OMe)-OMe, 8-9\% selectivity is lost for the formation of product $\mathbf{A}$ (Figure $4 \mathrm{a}$, entry 2). However, the product ratios are well within the range observed for other tetrameric peptides, reinforcing the observation that the $i+3$ residue is not the most influential position in guiding selectivity profiles (e.g., P18 and P19 in Figure 3). The dimer, Boc-Asp-Acpc$\mathrm{OMe}$, reveals a much more significant perturbation on product selectivity, providing approximately equal amounts of $\mathbf{A}, \mathbf{B}$ and $\mathbf{C}$ (entry 3), suggesting that a $\beta$-turn-biased structure of $\mathbf{P 1 0}$ may be the source of the observed preference for $\mathbf{A}$ in the optimal tetrameric peptides. Indeed, the dimer results in similar product distributions in comparison to the monomer, Boc-Asp-OMe (entry 4). All of these data, including insight from the crystal structures,

\section{Figure 4. Mechanistic considerations for A-selective catalyst $\mathbf{P 1 0}^{a, b}$}
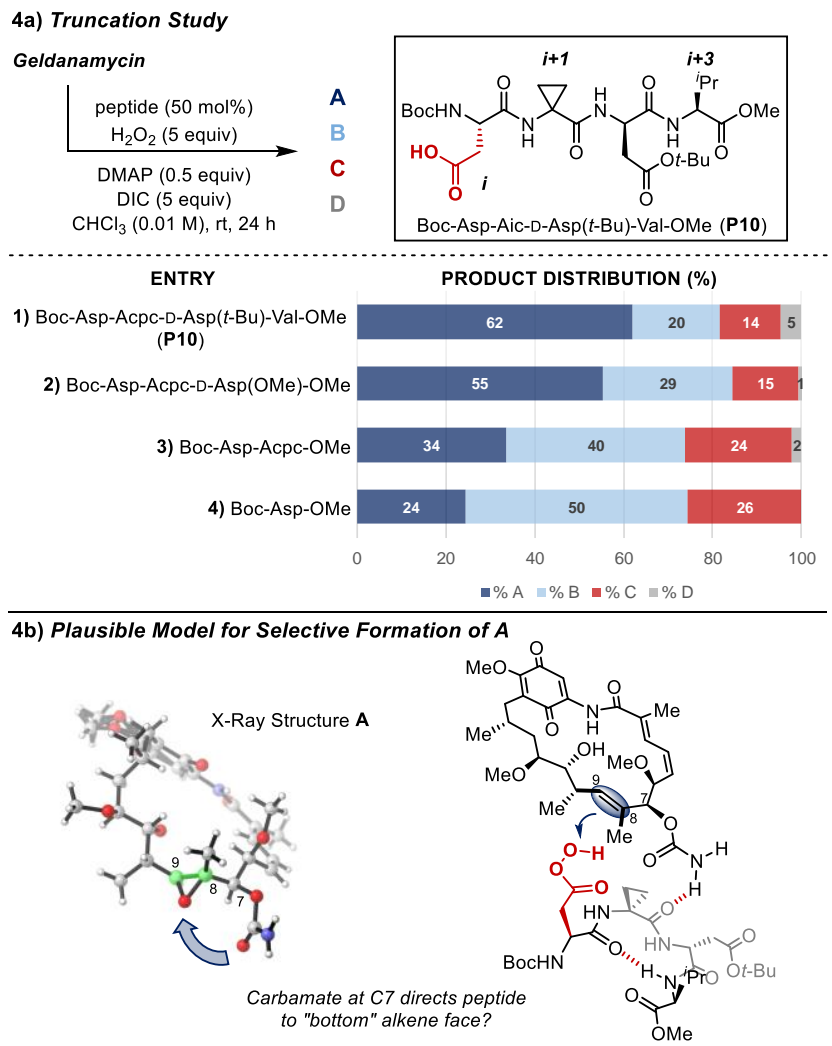

${ }^{\text {aStandard conditions: Geldanamycin }(2.8 \mathrm{mg}, 5 \mu \mathrm{mol}, 1 \text { equiv), peptide (50 }}$ mol \%), 4-dimethylaminopyridine (DMAP, $50 \mathrm{~mol} \%$ ), aqueous $\mathrm{H}_{2} \mathrm{O}_{2}(30$ wt \%; 5.0 equiv), $N, N^{\prime}$-diisopropylcarbodiimide (DIC, 5.0 equiv), $\mathrm{CHCl}_{3}$ $(0.01 \mathrm{M}), 22{ }^{\circ} \mathrm{C}, 24 \mathrm{~h} .{ }^{\mathrm{b}}$ Average from two trials. Determined using UPLC from crude reaction mixtures with an internal standard and calibration curve. Product distributions are corrected to total $100 \%$. See SI for yields. led us to consider a selectivity model, wherein the carbamate at $\mathrm{C} 7$ in geldanamycin could be engaged in a directing hydrogen-bond with the peptide backbone, guiding epoxidation to the "bottom" alkene face (Figure $4 b$ ).

The truncation study for $\mathbf{C}$-selective $\mathbf{P 1}$, which favors quinone epoxidation $(\mathbf{C}$ and $\mathbf{D})$, resulted in a much less drastic attenuation in product selectivity (Figure 5a). Surprisingly, each truncate resulted in similar product ratios to $\mathbf{P 1}$, all favoring the formation of $\mathbf{C}$ and D. Even the dimer Boc-Asp-Pro-OMe (entry 3) provides $\mathbf{C}$ and $\mathbf{D}$ in $69 \%$ and $27 \%$, respectively, in comparison to P1, yielding $\mathbf{C}$ and $\mathbf{D}$ in $76 \%$ and $22 \%$, respectively. A particularly remarkable comparison is that between two dimers, with only one residue changed: the dimer Boc-Asp-Acpc-OMe (Figure 4a, entry 3) favors 8,9-epoxidation overall $(74: 26=\mathbf{A}+\mathbf{B}: \mathbf{C}+\mathbf{D})$, while BocAsp-Pro-OMe remains selective for quinone oxidation $(4: 96=$ $\mathbf{A}+\mathbf{B}: \mathbf{C}+\mathbf{D}$ ). Thus, it appears that the first two residues of P1 (AspPro) are primarily responsible for directing the reaction towards the quinone epoxidation pathway.

Figure 5. Data-guided mechanistic speculation into quinone oxidation to form $\mathrm{C}$ and $\mathrm{D}^{a, b}$

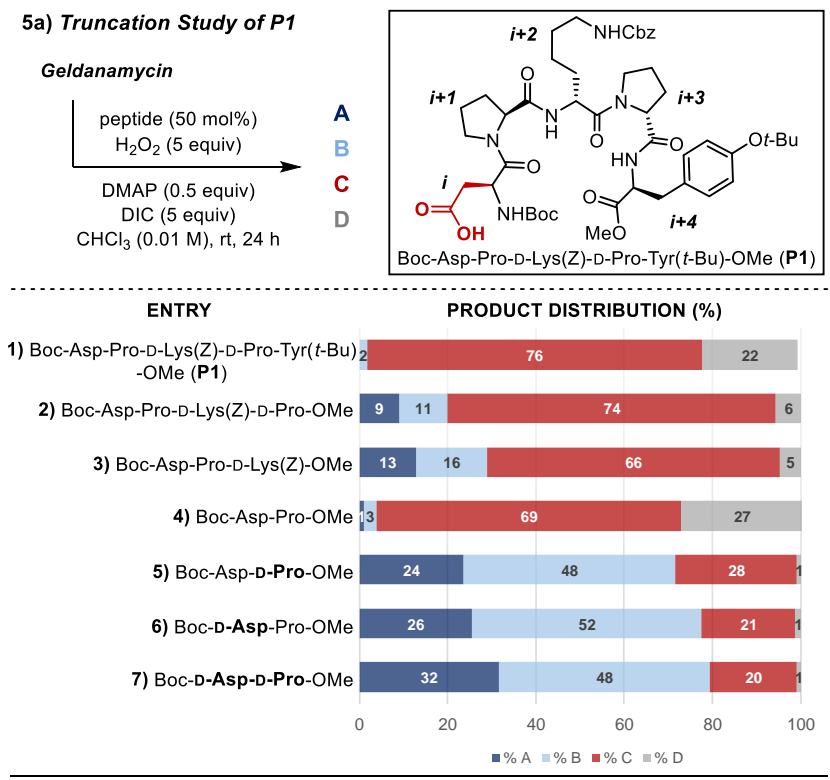

5b) Plausible Model for Selective Formation of C

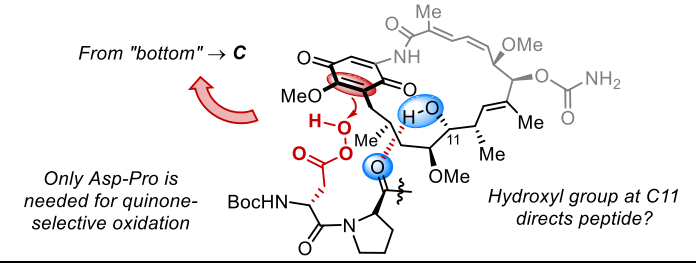

${ }^{\text {aStandard conditions: Geldanamycin }(2.8 \mathrm{mg}, 5 \mu \mathrm{mol}, 1 \text { equiv), peptide (50 }}$ mol \%), 4-dimethylaminopyridine (DMAP, 50 mol \%), aqueous $\mathrm{H}_{2} \mathrm{O}_{2}(30$ wt \%; 5.0 equiv), $N, N^{\prime}$-diisopropylcarbodiimide (DIC, 5.0 equiv), $\mathrm{CHCl}_{3}$ $(0.01 \mathrm{M}), 22{ }^{\circ} \mathrm{C}, 24 \mathrm{~h} .{ }^{\mathrm{b}}$ Average from two trials. Determined using UPLC from crude reaction mixtures with an internal standard and calibration curve. Product distributions are corrected to total $100 \%$. See SI for yields.

Notably, any perturbation to the L-Asp-L-Pro dipeptide stereochemistry supports this assertion (Figure 5a, entries 5-7). In each case, any other stereochemical dyad results in diversion of the reaction pathway towards 8,9-epoxidation (formation of $\mathbf{A}$ and $\mathbf{B}$ ). Furthermore, peptides containing the Asp-Pro sequence tend to provide a higher yield of products $\mathbf{C}$ and $\mathbf{D}$, but the overall product distribution is dependent on the remaining residues (e.g., P2, P4, P6, P15). The subtle yet complex influence of the peptide sequence on the reaction outcome reinforces the synergistic nature of attractive interactions between the substrate and catalyst. 
As quinone-selective catalyst $\mathbf{P} \mathbf{1}$ promotes a distinct reaction pathway, any selectivity model for the formation of $\mathbf{C}$ and $\mathbf{D}$ will differ substantially from that shown in Figure $4 \mathrm{~b}$. Thus, shown in Figure $5 \mathrm{~b}$ is an alternative scenario that may account for the strict requirement for L-Asp-L-Pro chirality, with further selectivity tuning by distal residues. Accordingly, it is possible that the peracid and Pro carbonyl (blue circle) are oriented in the same direction, ${ }^{29}$ such that interactions with the $\operatorname{Pro}(\mathrm{C}=\mathrm{O})$ are responsible for this selectivity. The hydroxyl group at $\mathrm{C} 11$, which is situated near the back of the quinone, as drawn, could be involved in directing the peptide via hydrogen-bonding with $\operatorname{Pro}(\mathrm{C}=\mathrm{O})$ towards product $\mathbf{C}$. Formation of the minor epoxyquinone $\mathbf{D}$ could then result from conformational inversion of the quinone $\pi$-face, or even be directed by hydrogen-bonding with the amide at $\mathrm{C} 1$.

Regarding the plausible selectivity models presented in Figures $4 \mathrm{~b}$ and $5 \mathrm{~b}$, it is absolutely essential to acknowledge that mechanistic rationalizations for selectivity outcomes with ratios at these levels must be realized with an abundance of mechanistic caveats. However, at the global level of these observed reversals of product selectivity, in situations in which multiple, nonstereoisomeric products are formed, we contend that the state of the art is limited and that these heuristic models may guide the field forward. Computational studies (and even empirical studies ${ }^{29,30}$ ) of problems of this complex nature are also a frontier-level, extensive endeavor, ${ }^{31-33}$ and ongoing efforts along these lines will no doubt add further insight.

As we did not anticipate formation of the epoxyquinones $\mathbf{C}$ and $\mathbf{D}$ from these studies, we wondered if their formation was a particular function of the catalysts, the secondary structure of geldanamycin and its epoxyquinone adducts, or a synergistic result of both features. Therefore, we examined the reactions of several model quinones (3-6; Figure 6) under analogous conditions. In all cases, no detectable reactions were observed using $m$-CPBA, and decomposition of the model quinones was detected under standard reaction conditions with $\mathbf{P 1}$, or when nucleophilic epoxidation conditions were tested (e.g., $t \mathrm{BuOOH}$, base). In contrast, compounds $\mathbf{C}$ and $\mathbf{D}$ seem to be quite stable when formed under conditions of catalytic P1. These realizations further bolster the symbiotic relationship between these catalysts and their substrates. These findings may well suggest that $\mathbf{P 1}$, originally discovered as a nucleophilic peracid and Baeyer-Villiger oxidation catalyst, ${ }^{8}$ may well be functioning once again in a peptide-tuned nucleophilic manner in the presence of the specific functionality-adorned geldanamycin scaffold. This assertion is supported by the fact that, subsequent to the discovery of $\mathbf{P 1}$, and based on its behavior, we also found the classical, stoichiometric nucleophilic epoxidation conditions ( $t \mathrm{BuOOH}$, base; see SI for details) allow the formation of $\mathbf{C}$ and $\mathbf{D}$ with yields and selectivities comparable to catalytic P1. Since these conditions fail to deliver efficient epoxidation of the model quinones further underscores the importance of the global functionality of the natural product, rather than simply its local structural environment.

\section{Figure 6. Evaluation of Model Quinones}
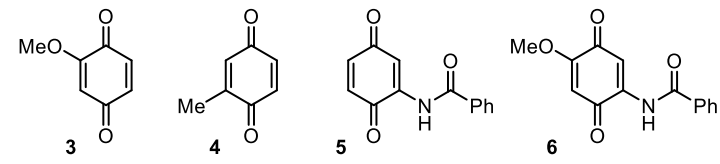

with $m$-CPBA, no reaction with P1, decomposition

Having identified the selective peptide catalysts P10 and P1 for geldanamycin oxidation, and in pursuit of ample quantities for characterization and biological evaluation, several optimized experiments were carried out (Scheme 1). Fortuitously, higher reactivity was observed as a function of higher reagent loadings/lower peptide catalyst loadings. With P10, $\mathbf{A}$ and $\mathbf{B}$ were favored in slightly higher ratios than at the microscale, resulting in a 3.9:1 dr of A:B (74:19:6:1 of A:B:C:D). Products A and $\mathbf{B}$ were isolated in $39 \%$ and $10 \%$ yields, respectively. With P1, higher conversion could be achieved through use of stoichiometric DMAP, resulting in an A:B:C:D ratio of 0:0:76:24, from which $\mathbf{C}$ and $\mathbf{D}$ were isolated in $32 \%$ and $14 \%$ yields, respectively. Taken together, a comparison of catalysts $\mathbf{P 1 0}$ to $\mathbf{P 1}$ on somewhat larger scale reveals that the $\mathrm{C} 8,9(\mathbf{A}+\mathbf{B})$ versus $\mathrm{C} 16,17 \quad(\mathbf{C}+\mathbf{D})$ functionalization ratios can swing from 93:7 to 0:100 as a function of the peptide-based catalyst, of course with attendant tuning of the diastereoselectivity in each series.

\section{Scheme 1. Isolation of geldanamycin analogues}

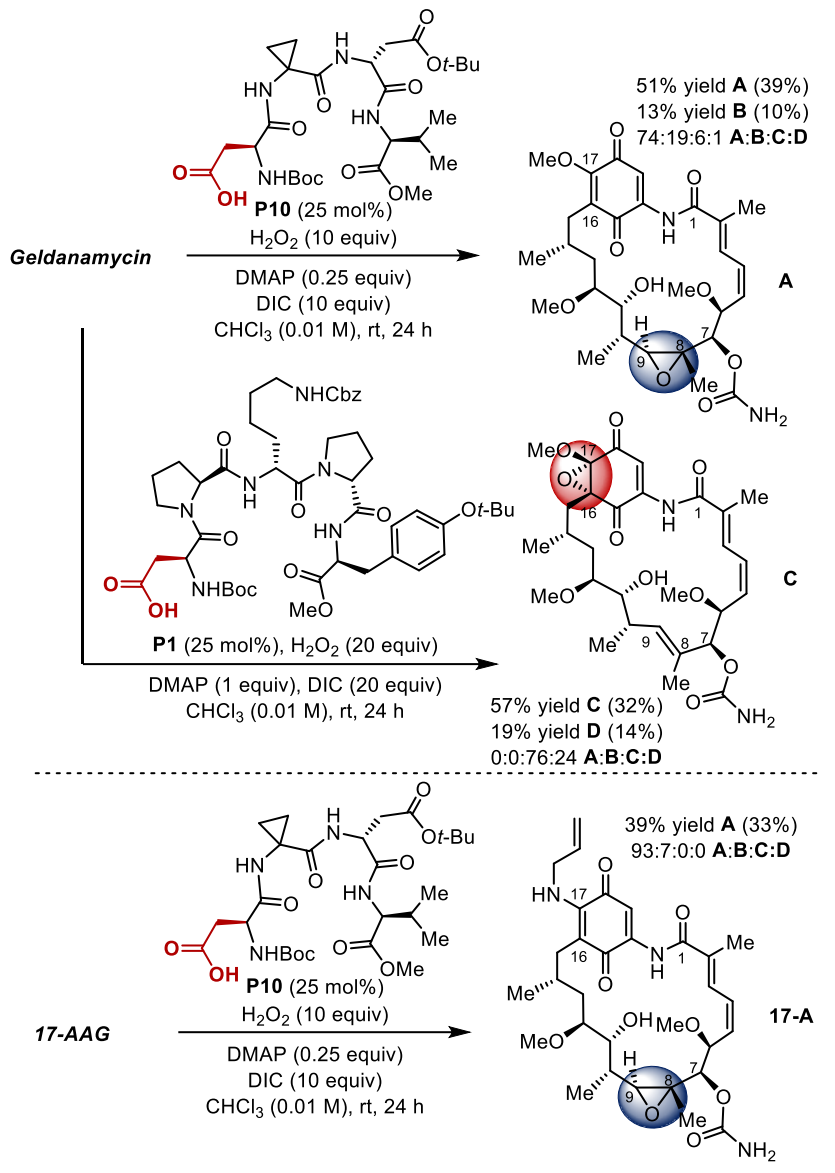

As 17-substituted geldanamycin derivatives have demonstrated improved bioactivity and bioavailability, ${ }^{23,34,35}$ we sought to test these optimal peptide sequences and reaction conditions with 17-AAG to access additional analogs. We predicted that P10 would likely lead to the same mode of epoxidation, as the local environment near the 8,9-position is unchanged. However, we were uncertain if adjusting the electronic nature of the quinone would disrupt or enhance quinone epoxidation with $\mathbf{P 1}$. With the scaled reaction conditions shown in Scheme 1, P10 indeed affords the expected product, in fact with elevated selectivity compared to geldanamycin, resulting in 17-A in $93 \%$ selectivity and $33 \%$ isolated yield. The same epoxide diastereomer as in $\mathbf{A}$ was confirmed by X-ray crystallography analysis (See SI). Yet, $m$-CPBA was less effective with 17-AAG than geldanamycin, providing only $30 \%$ conversion and favoring the same product as $\mathbf{P 1 0}$ in a 2:1 ratio (See SI). Finally, reaction of 17-AAG with P1 resulted in only $10 \%$ conversion of the starting material and detection of one new mono-oxidized product by UPLC-MS (See SI). While we have not definitively assigned the structure of this compound, the low reactivity of this modified 
quinone core in 17-AAG reflects the significant influence of the substituents on accessing quinone oxidation. No further optimization or isolation of this product was attempted.

Biology. Intrigued by these novel epoxy-geldanamycin and 17-AAG analogues, their biological activity was investigated. As previously mentioned, geldanamycin is a well characterized Hsp90 inhibitor and has shown promising anticancer activity. Therefore, the anti-proliferative activity manifested by these analogs was determined against two breast cancer cell lines, MCF-7 and SKBr3. Only one of the new compounds inhibited the growth of these cancer cells at $10 \mu \mathrm{M}$. Compound $\mathbf{B}$, which resulted from the reaction of geldanamycin and $m$-CPBA, retained the majority of the parent compound's anti-proliferative activity. In fact, compound $\mathbf{B}$ manifested an $\mathrm{EC}_{50}$ value of $338.1 \pm 160.2$ and $28.7 \pm 14.3 \mathrm{nM}$ against MCF-7 and SKBr-3 cell lines, respectively. This activity was similar (albeit less active) to geldanamycin's EC 50 values of 19.9 and $24 \mathrm{nM}$, respectively (NCI ID: 122750).

In addition to the anti-proliferative activity manifested by these compounds, the molecules were evaluated for direct binding to Hsp90 isoforms. The affinity of these epoxide analogues was determined for the individual isoforms of Hsp90 to establish whether any selectivity resulted from this modification. Fluorescence polarization assays have revealed geldanamycin to exhibit a high affinity towards three of the four isoforms (Hsp90 $\alpha$, Hsp903, Grp94), as compared to the mitochondrial localized isoform, TRAP1. ${ }^{15}$ Therefore, the affinity of these molecules for Hsp90 $\alpha$, Hsp90 $\beta$, and Grp94 was determined using a fluorescence polarization assay. The results are summarized in Table 1 .

Table 1. Epoxy Analogues Apparent Affinity Data

$$
\mathbf{K}_{\mathbf{D}}(\boldsymbol{\mu M})
$$

\begin{tabular}{|c|c|c|c|}
\hline CMPD & Hsp90a & Hsp90ß & Grp94 \\
\hline $\mathbf{A}$ & $3.41 \pm 0.60$ & $1.08 \pm 0.073$ & $2.55 \pm 0.46$ \\
\hline B & $0.083 \pm 0.029$ & $0.054 \pm 0.0001$ & $0.13 \pm 0.07$ \\
\hline $\mathbf{C}$ & $0.76 \pm 0.26$ & $0.395 \pm 0.053$ & $1.10 \pm 0.49$ \\
\hline D & $0.32 \pm 0.09$ & $0.24 \pm 0.04$ & $0.54 \pm 0.09$ \\
\hline 17-A & $>10$ & $>10$ & $>10$ \\
\hline 17-B & $0.90 \pm 0.38$ & $0.81 \pm 0.09$ & $1.13 \pm 0.02$ \\
\hline
\end{tabular}

Consistent with the anti-proliferative data, compound $\mathbf{B}$ displayed a high affinity for all three Hsp90 isoforms. Compared to diastereomer A, compound $\mathbf{B}$ manifested $\sim 20$ x's higher affinity. Intriguingly, compounds $\mathbf{C}$ and $\mathbf{D}$ exhibited no anti-proliferative activity, but retained sub-micromolar affinity towards Hsp90.

In order to validate that compound $\mathbf{B}$ manifests its antiproliferative activity via Hsp90 inhibition, the ability of compound B to induce client protein degradation was investigated. Client protein degradation occurs as a result of Hsp90 inhibition, because the protein substrate is unable to reach conformation maturation and instead, is degraded via the ubiquitin-proteasome pathway. Therefore, SKBr-3 cells were treated with increasing concentrations of compound $\mathbf{B}$ and select Hsp90-dependent client proteins were evaluated via Western-blot analysis (Figure 7). Consistent with Hsp90 inhibition, the EGF receptor and HER2 levels were reduced in a dose-dependent manner. In addition, treatment of cells with geldanamycin is known to induce the heat shock response, which results in the increased expression of Hsp70 and/or Hsp90. ${ }^{16}$ As shown in Figure 7, compound $\mathbf{B}$ induced a dose-dependent increase in the expression of Hsp70 and Hsp90, consistent with Hsp90 inhibition in the cellular environment.
Figure 7. Compound B Client Protein Degradation Assay

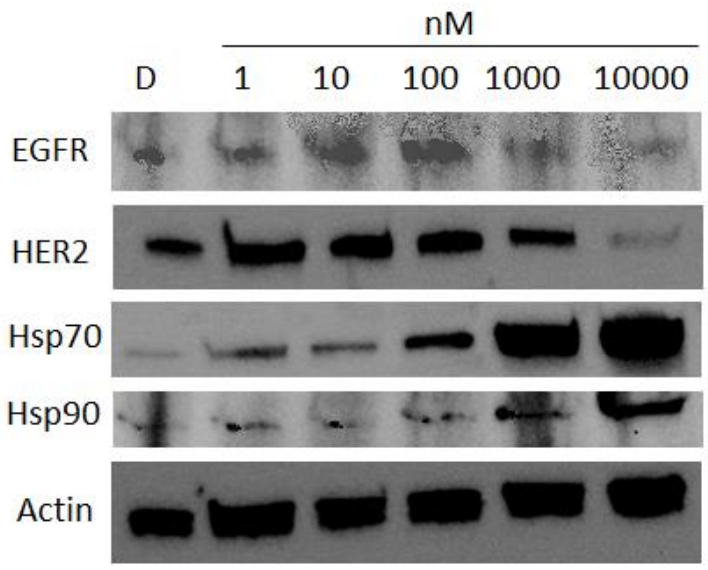

Historically, strategies to diversify geldanamycin have focused on the quinone moiety. However, these results suggest that it may be advantageous to develop analogues of geldanamycin through innovative chemical diversity methods such as that described herein, which provided access to analogues that were otherwise unobtainable.

\section{CONCLUSIONS}

These studies provide significant evidence that peptide modified catalytic functionalities can deliver dramatic reversals of stereo-, site- and functional group selectivity in complex molecular settings. Complementary to studies of site-selective natural product diversification in settings where explicit targeting strategies may be derived, for example the glycopeptide antibiotics, ${ }^{36-39}$ this report shows the generality of these ideas for application to both a new natural product scaffold (geldanamycin), and to a reaction class that might have seemed recalcitrant to the approach (functional group-selective epoxidation). Unique chemical discoveries emerged that highlight a potentially fertile intersection for discovery when the domains of complex catalysts and complex substrates are compelled to interact. Certainly in this case, the new products formed, and possibly the selective formation of previously unassigned geldanamycin oxides, would have been elusive absent the diversification of the geldanamycin scaffold with catalysts targeting outer sphere interactions.

Selective access to samples of $\mathbf{A}, \mathbf{B}, \mathbf{C}$ and $\mathbf{D}$ also delivered new information about the biological potential resident in these new derivatives, and present an enigma worthy of further study that the quinone analogs $\mathbf{C}$ and $\mathbf{D}$ maintain Hsp90 affinity, while losing anti-proliferative effects. ${ }^{40}$ Accordingly, to the extent that these chemical studies unveiled previously unknown, and resolved previously unassigned, geldanamycin analogs, perhaps these studies have also added incentive to search for additional presently cryptic catalyst-enabled structures, creating opportunities for resolving additional cryptic biological ambiguities.

\section{ASSOCIATED CONTENT}

Supporting Information.

The Supporting Information is available free of charge on the ACS Publications website at DOI:

\section{AUTHOR INFORMATION}

\section{Corresponding Author}

*E-mail: scott.miller@yale.edu

\section{ORCID}


Scott J. Miller: 0000-0001-7817-1318

Margaret J. Hilton: 0000-0003-1843-2322

Brian S. J. Blagg: 0000-0002-6200-3480

\section{Notes}

The authors declare no competing financial interest.

\section{ACKNOWLEDGMENT}

S.J.M. is grateful to the National Institute of General Medical Sciences (NIGMS) of the NIH R35 GM132092). M.J.H. would like to thank the NIGMS of the NIH for support (F32GM125119). We thank Dr. Yu Tang and Dr. Sheng-Ying Hsieh for sharing peptide catalysts (Yale). B.S.J.B. is grateful to the NIH for financial support (CA213566).

\section{REFERENCES}

(1) Wolfenden, R.; Snider, M. J. The Depth of Chemical Time and the Power of Enzymes as Catalysts. Acc. Chem. Res. 2001, 34, 938. (2) Benkovic, S. J.; Hammes-Schiffer, S. A Perspective on Enzyme Catalysis. Science 2003, 301, 1196.

(3) Trost, B. M. Asymmetric catalysis: An enabling science. Proc. Natl. Acad. Sci. U. S. A. 2004, 101, 5348.

(4) Shugrue, C. R.; Miller, S. J. Applications of Nonenzymatic Catalysts to the Alteration of Natural Products. Chem. Rev. 2017, 117, 11894.

(5) Robles, O.; Romo, D. Chemo- and site-selective derivatizations of natural products enabling biological studies. Nat. Prod. Rep. 2014, 31, 318.

(6) Peris, G.; Jakobsche, C. E.; Miller, S. J. Aspartate-Catalyzed Asymmetric Epoxidation Reactions. J. Am. Chem. Soc. 2007, 129, 8710.

(7) Lichtor, P. A.; Miller, S. J. Combinatorial evolution of site- and enantioselective catalysts for polyene epoxidation. Nat. Chem 2012, 4, 990

(8) Romney, D. K.; Colvin, S. M.; Miller, S. J. Catalyst Control over Regio- and Enantioselectivity in Baeyer-Villiger Oxidations of Functionalized Ketones. J. Am. Chem. Soc. 2014, 136, 14019.

(9) Alford, J. S.; Abascal, N. C.; Shugrue, C. R.; Colvin, S. M.; Romney, D. K.; Miller, S. J. Aspartyl Oxidation Catalysts That Dial In Functional Group Selectivity, along with Regio- and Stereoselectivity. ACS Cent. Sci. 2016, 2, 733.

(10) Whitesell, L.; Mimnaugh, E. G.; De Costa, B.; Myers, C. E.; Neckers, L. M. Inhibition of heat shock protein HSP90-pp60v-src heteroprotein complex formation by benzoquinone ansamycins: essential role for stress proteins in oncogenic transformation. Proc. Natl. Acad. Sci. U. S. A. 1994, 91, 8324.

(11) Prodromou, C.; Roe, S. M.; O'Brien, R.; Ladbury, J. E.; Piper, P. W.; Pearl, L. H. Identification and Structural Characterization of the ATP/ADP-Binding Site in the Hsp90 Molecular Chaperone. Cell 1997, 90, 65.

(12) Li, L.; Wang, L.; You, Q.-D.; Xu, X.-L. Heat Shock Protein 90 Inhibitors: An Update on Achievements, Challenges, and Future Directions. J. Med. Chem. 2019, DOI: 10.1021/acs.jmedchem.9b00940.

(13) Taldone, T.; Sun, W.; Chiosis, G. Discovery and development of heat shock protein 90 inhibitors. Bioorg. Med. Chem. 2009, 17, 2225.

(14) Franke, J.; Eichner, S.; Zeilinger, C.; Kirschning, A. Targeting heat-shock-protein 90 (Hsp90) by natural products: geldanamycin, a show case in cancer therapy. Nat. Prod. Rep. 2013, 30, 1299.

(15) Chaudhury, S.; Welch, T. R.; Blagg, B. S. J. Hsp90 as a Target for Drug Development. ChemMedChem 2006, 1, 1331.

(16) J. G. Supko, R. L. H., M. R. Grever, L. Malspeis. Preclinical pharmacologic evaluation of geldanamycin as an antitumor agent. Cancer Chemother. Pharmacol. 1995, 36, 305.

(17) For total syntheses of geldanamycin, see: a) Qin, H.-L.; Panek,
J. S. Total Synthesis of the Hsp90 Inhibitor Geldanamycin. Org. Lett. 2008, 10, 2477; b) Andrus, M. B.; Meredith, E. L.; Hicken, E. J.; Simmons, B. L.; Glancey, R. R.; Ma, W. Total Synthesis of (+)Geldanamycin and (-)-o-Quinogeldanamycin: Asymmetric Glycolate Aldol Reactions and Biological Evaluation. J. Org. Chem. 2003, 68, 8162.

(18) For a recent contribution describing total synthesis-based analog syntheses with an extensive bibliography, see: Jin, L.; Zhang, R.; Zhang, Z.; Bian, C.; Yu, X. Total synthesis based modification of benzoquinone ansamycin antibiotics: $\mathrm{C} 8$ diversification of C5-C15 fragments. Tet. Lett. 2019, 60, 547.

(19) For examples of biosynthetic analogs of geldanamycin, see: a) Patel, K.; Piagentini, M.; Rascher, A.; Tian, Z.-Q.; Buchanan, G. O.; Regentin, R.; Hu, Z.; Hutchinson, C. R.; McDaniel, R. Engineered Biosynthesis of Geldanamycin Analogs for Hsp90 Inhibition. Chem. Biol. 2004, 11, 1625; b) Buchanan, G. O.; Regentin, R.; Piagentini, M.; Rascher, A.; McDaniel, R.; Galazzo, J. L.; Licari, P. J. Production of 8-Demethylgeldanamycin and 4,5Epoxy-8-demethylgeldanamycin from a Recombinant Strain of Streptomyces hygroscopicus. J. Nat. Prod. 2005, 68, 607.

(20) Khandelwal, A.; Crowley, V. M.; Blagg, B. S. J. Natural Product Inspired N-Terminal Hsp90 Inhibitors: From Bench to Bedside? Med. Res. Rev. 2016, 36, 92.

(21) Kitson, R. R. A.; Moody, C. J. Learning from Nature: Advances in Geldanamycin- and Radicicol-Based Inhibitors of Hsp90. J. Org. Chem. 2013, 78, 5117.

(22) Yñigez-Gutierrez, A. E.; Bachmann, B. O. Fixing the Unfixable: The Art of Optimizing Natural Products for Human Medicine. J. Med. Chem. 2019, 62, 8412.

(23) Schulte, T. W.; Neckers, L. M. The benzoquinone ansamycin 17-allylamino-17-demethoxygeldanamycin binds to HSP90 and shares important biologic activities with geldanamycin. Cancer Chemother. Pharmacol. 1998, 42, 273.

(24) Biamonte, M. A.; Van de Water, R.; Arndt, J. W.; Scannevin, R. H.; Perret, D.; Lee, W.-C. Heat Shock Protein 90: Inhibitors in Clinical Trials. J. Med. Chem. 2010, 53, 3.

(25) Henbest, H. B.; Wilson, R. A. L. 376. Aspects of stereochemistry. Part I. Stereospecificity in formation of epoxides from cyclic allylic alcohols. J. Chem. Soc. 1957, 1958.

(26) Haque, T. S.; Little, J. C.; Gellman, S. H. Stereochemical Requirements for $\beta$-Hairpin Formation: Model Studies with FourResidue Peptides and Depsipeptides. J. Am. Chem. Soc. 1996, 118, 6975.

(27) Gunasekaran, K.; Gomathi, L.; Ramakrishnan, C.; Chandrasekhar, J.; Balaram, P. Conformational interconversions in peptide $\beta$-turns: analysis of turns in proteins and computational estimates of barriers. J. Mol. Biol. 1998, 284, 1505.

(28) Wilmot, C. M.; Thornton, J. M. Analysis and prediction of the different types of $\beta$-turn in proteins. J. Mol. Biol. 1988, 203, 221.

(29) Abascal, N. C.; Miller, S. J. Solution Structures and Molecular Associations of a Peptide-Based Catalyst for the Stereoselective Baeyer-Villiger Oxidation. Org. Lett. 2016, 18, 4646.

(30) Rigling, C.; Kisunzu, J. K.; Duschmalé, J.; Häussinger, D.; Wiesner, M.; Ebert, M.-O.; Wennemers, H. Conformational Properties of a Peptidic Catalyst: Insights from NMR Spectroscopic Studies. J. Am. Chem. Soc. 2018, 140, 10829.

(31) Yan, X. C.; Metrano, A. J.; Robertson, M. J.; Abascal, N. C.; Tirado-Rives, J.; Miller, S. J.; Jorgensen, W. L. Molecular Dynamics Simulations of a Conformationally Mobile PeptideBased Catalyst for Atroposelective Bromination. ACS Catal. 2018, $8,9968$.

(32) Thiel, W. Computational Catalysis - Past, Present, and Future. Angew. Chem. Int. Ed. 2014, 53, 8605.

(33) Lam, Y.-H.; Grayson, M. N.; Holland, M. C.; Simon, A.; Houk, K. N. Theory and Modeling of Asymmetric Catalytic Reactions. Acc. Chem. Res. 2016, 49, 750.

(34) Mellatyar, H.; Talaei, S.; Pilehvar-Soltanahmadi, Y.; 
Barzegar, A.; Akbarzadeh, A.; Shahabi, A.; Barekati-Mowahed, M.; Zarghami, N. Targeted cancer therapy through 17-DMAG as an Hsp90 inhibitor: Overview and current state of the art. Biomed. Pharmacother. 2018, 102, 608.

(35) Tian, Z.-Q.; Liu, Y.; Zhang, D.; Wang, Z.; Dong, S. D.; Carreras, C. W.; Zhou, Y.; Rastelli, G.; Santi, D. V.; Myles, D. C. Synthesis and biological activities of novel 17-aminogeldanamycin derivatives. Bioorg. Med. Chem. 2004, 12, 5317.

(36) Han, S.; Miller, S. J. Asymmetric Catalysis at a Distance: Catalytic, Site-Selective Phosphorylation of Teicoplanin. J. Am. Chem. Soc. 2013, 135, 12414.

(37) Pathak, T. P.; Miller, S. J. Site-Selective Bromination of Vancomycin. J. Am. Chem. Soc. 2012, 134, 6120.

(38) Fowler, B. S.; Laemmerhold, K. M.; Miller, S. J. Catalytic Site-Selective Thiocarbonylations and Deoxygenations of Vancomycin Reveal Hydroxyl-Dependent Conformational Effects. J. Am. Chem. Soc. 2012, 134, 9755.

(39) Pathak, T. P.; Miller, S. J. Chemical Tailoring of Teicoplanin with Site-Selective Reactions. J. Am. Chem. Soc. 2013, 135, 8415.

(40) Chiosis, G.; Neckers, L. Tumor Selectivity of Hsp90 Inhibitors: The Explanation Remains Elusive. ACS Chem. Biol. 2006, 1, 279. 


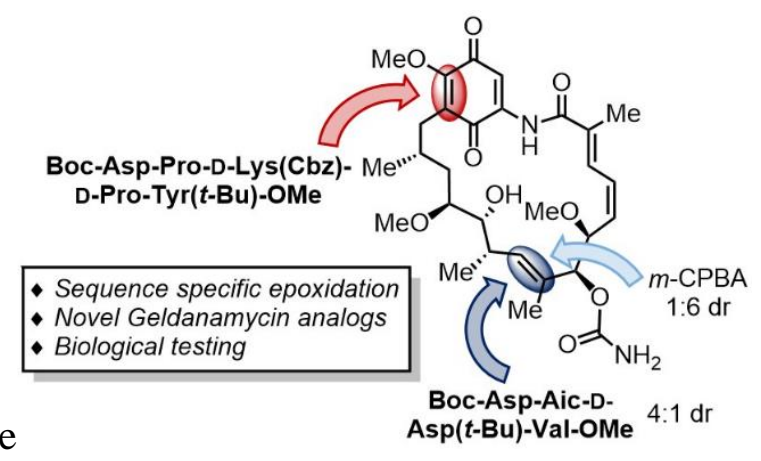

Insert Table of Contents artwork here

Boc-Asp-Aic-D-
Asp(t-Bu)-Val-OMe 


\section{Catalysis-Enabled Access to Cryptic Geldanamycin Oxides}

Margaret J. Hilton, ${ }^{\dagger}$ Christopher M. Brackett,,${ }^{\ddagger}$ Brandon Q. Mercado, ${ }^{\dagger}$ Brian S. J. Blagg, ${ }^{\ddagger}$ Scott J. Miller ${ }^{\dagger *}$

${ }^{\dagger}$ Department of Chemistry, Yale University, New Haven, Connecticut 06520, United States

¥Department of Chemistry and Biochemistry, University of Notre Dame, Notre Dame, Indiana 46556, United States

\section{Supporting Information}

\section{Table of Contents}

I. General Information 2

II. Peptide Synthesis 3

III. $\quad$ Peptide Screening Conditions and Results 8

IV. Large Scale Reaction Conditions 10

V. Liquid Chromatography Chromatograms 12

$\begin{array}{ll}\text { VI. Model Quinone Synthesis and Reactions } & 18\end{array}$

$\begin{array}{ll}\text { VII. } \quad \text { Biological Testing } & 19\end{array}$

$\begin{array}{ll}\text { VIII. NMR Spectra } & 20\end{array}$

IX. $\quad$ X-Ray Crystallography Data 26

$\begin{array}{ll}X . & \text { References } \\ & 105\end{array}$ 


\section{General Information}

All reactions were carried out under benchtop conditions, without exclusion of air or moisture, unless otherwise stated. Room temperature is considered $20-23^{\circ} \mathrm{C}$. All reagents were obtained from commercial suppliers and used as received, without further purification, unless otherwise stated. Geldanamycin and 17-AAG were purchased from Alfa Aesar and used as received. THF, $\mathrm{CH}_{2} \mathrm{Cl}_{2}$, DMF, and PhMe were dried over alumina and dispensed under argon from a Seca Solvent purification system by GlassContour. Triethylamine $\left(\mathrm{Et}_{3} \mathrm{~N}\right), \mathrm{N}, \mathrm{N}$ '-diisopropyl ethylamine (i-Pr ${ }_{2} \mathrm{NEt}$ ), and $\mathrm{N}, \mathrm{N}$-diisopropylamine $\left(\mathrm{i}-\mathrm{Pr}_{2} \mathrm{NH}\right)$ were distilled over $\mathrm{CaH}$ under a nitrogen atmosphere prior to use. Deionized water was used for reactions, extractions, and RP chromatography. HPLC grade solvents were used for all other chromatography.

Unless otherwise stated, all NMR data were acquired at ambient temperature. NMR solvents, were purchased from Cambridge Isotopes and used as received. NMR spectra were processed with MestReNova x64-12.0.1-20560 software using the baseline and phasing correction features. Multiplicities and coupling constants were calculated using the multiplet analysis feature with manual intervention as necessary. ${ }^{1} \mathrm{H}$ NMR spectra were obtained on Agilent $400 \mathrm{MHz}, 500 \mathrm{MHz}$ or $600 \mathrm{MHz}$ spectrometers. Proton chemical shifts $(\delta)$ are reported in ppm and referenced to residual solvent peaks for $\mathrm{CDCl}_{3}(\delta 7.26 \mathrm{ppm})$. Proton data are reported as chemical shift, (multiplicity [singlet (s), doublet (d), triplet (t), quartet $(q)$, pentet (p), heptet (hept), multiplet $(m)$, broad singlet (bs), doublet of doublets (dd), doublet of doublet of doublets (ddd), doublet of doublet of triplets (ddt), doublet of triplets (dt), doublet of triplet of triplets (dtt), etc. and apparent (app)] coupling constants [Hz], and integrations). ${ }^{13} \mathrm{C}$ NMR spectra were obtained on Agilent 500 (125 $\mathrm{MHz}) \mathrm{MHz}$ or 600 (150 MHz) MHz spectrometers with full proton decoupling. Carbon chemical shifts $(\delta)$ are reported in ppm and referenced to residual solvent peaks for $\mathrm{CDCl}_{3}(\delta 77.2 \mathrm{ppm})$. Analytical thin-layer chromatography (TLC) was performed using EMD Millipore silica gel 60 F254 precoated plates $(0.25 \mathrm{~mm}$ thickness) and developed plates were visualized using a UV lamp and/or potassium permanganate $\left(\mathrm{KMnO}_{4}\right)$ stain. TLC $\mathrm{R}_{f}$ values are reported.

Normal-phase flash-column chromatography was performed using an automated Biotage Isolera One flash purification system equipped with a 10, 25, or $50 \mathrm{~g}$ SNAP Ultra (HP Sphere, $25 \mu \mathrm{m}$ silica) cartridge. Reversed-phase flash-column chromatography was performed using an automated Biotage Isolera One flash purification system equipped with a 12, 30, 60 or $120 \mathrm{~g}$ SNAP C-18 (HS $50 \mu \mathrm{m}$ silica) or SNAP Ultra C-18 (HP Sphere, $25 \mu \mathrm{m}$ silica) cartridge. Preparative reverse phase HPLC was performed using a Shimadzu Prominence system equipped with LC20AP pumps, CBM-20A Communications BUS module, SPD-20A UV/vis detector, SIL-10AP autosampler, and FRC-10A fractions collector. Ultra-high performance liquid chromatographymass spectrometry (UPLC/MS) was performed on a Waters Acquity SQD2 instrument equipped with an CORTECS C18 column (1.6 $\mu \mathrm{m}$ particle size, $3.0 \times 50 \mathrm{~mm})$ and an Ultra BEH C-18 column (1.7 $\mu \mathrm{m}$ particle size, 2.1 x $50 \mathrm{~mm}$ ), a dual atmospheric pressure chemical ionization (API)/electrospray ionization (ESI) mass spectrometry detector, and a photodiode array detector. High-resolution mass spectrometry (HRMS) was conducted by the service of Chemical and Biophysical Instrumentation Center in the chemistry department at Yale University on a Waters Xevo Q-TOF high-resolution Mass Spectrometry using ESI. Infrared spectra were recorded on a Nicolet 6700 ATR/FT-ATR spectrometer, and $v_{\max }$ are partially reported in $\mathrm{cm}^{-1}$. Optical rotations were recorded on a Autopol VI Automatic Polarimeter at the sodium D-line (589 nm) using a Type

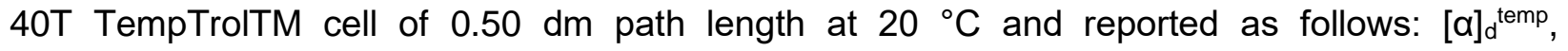
concentration ( $c$ in $\mathrm{g} / 100 \mathrm{~mL}$ ), and solvent. 


\section{Abbreviations:}

$A c=$ acetyl; Acpc = 1-aminocyclopropane-1-carboxylic acid; Aib = a-aminoisobutyric acid; Aic = 2aminoindane-2-carboxylic acid; Boc = tert-butoxycarbonyl; Bn = benzyl; $\mathrm{Bz}=$ benzoyl; $\mathrm{Cbz}=$ carboxybenzyl; Dap = 2,3-diaminoproprionic acid; DIC = N,N-diisopropylcarbodiimide; DMAP = 4-dimethylaminopyridine; $\quad \mathrm{DMF}=N, N$-dimethylformamide; $\quad \mathrm{EDC} \cdot \mathrm{HCl}=\mathrm{N}$-(3Dimethylaminopropyl)-Nethylcarbodiimide hydrochloride; ESI = electrospray ionization; EtOAc = ethyl acetate; Fmoc = 9-fluorenylmethoxycarbonyl; HATU = O-(7-azabenzotriazol-1-yl)- N,N,N,Ntetramethyluronium hexafluorophosphate; HBTU $=$ O-(Benzotriazol-1-yl)- $N, N, N, N-$ tetramethyluronium hexafluorophosphate; HCTU = 2-(6-chloro-1-H-benzotriazole-1-yl)-1,1,3,3tetramethylaminium hexafluorophosphate $\mathrm{HOBt}=1$-hydroxybenzotriazole; HPLC = highperformance liquid chromatography; HRMS = high-resolution mass spectrometry; IR = infrared; UPLC-MS = ultraperformance liquid chromatography mass spectrometry; $\mathrm{NMM}=4$ methylmorpholine; NMR = nuclear magnetic resonance; $\mathrm{RP}=$ reversed-phase; $\mathrm{rt}=$ room temperature; TFA = trifluoroacetic acid; THF = tetrahydrofuran; TLC = thin-layer chromatography; TOF $=$ time-of-flight.

\section{Peptide Synthesis}

1. General Procedure A: Solid Phase Peptide Synthesis

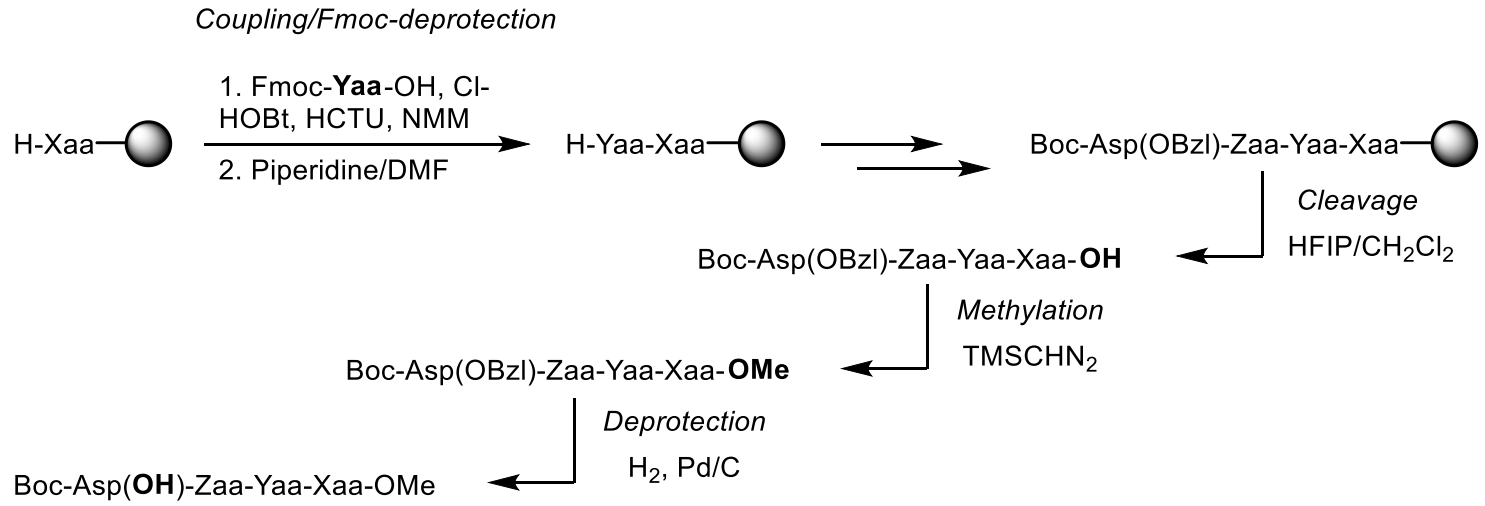

\section{Coupling and Fmoc-Deprotection}

First, 2-chlorotrityl resins ( $\mathrm{H}$-Xaa-2-ClTrt-Resin, 1 equiv) were swelled in $\mathrm{CH}_{2} \mathrm{Cl}_{2}$ for 20 min. Then, the appropriate amino acid monomers (Fmoc-Yaa-OH, 3 equiv) were coupled using $\mathrm{Cl}-\mathrm{HOBt}(3$ equiv), HCTU (3 equiv), and $N$-methylmorpholine (6 equiv) in $N$-methyl-2-pyrrolidone (NMP). The amino acid, coupling reagents, and base were premixed before adding to the solid support. Couplings generally proceeded for 3 to $5 \mathrm{~h}$. Deprotections were achieved with $20 \%(\mathrm{v} / \mathrm{v})$ piperidine in DMF for approximately 20 min. The solid phase was rinsed thoroughly with $\mathrm{CH}_{2} \mathrm{Cl}_{2}$ and DMF between each coupling and deprotection step. Coupling and deprotections steps proceeded until the desired $N$-terminal residue is reached (in most cases, Boc-Asp(OBzl)-OH, 3 equiv). 


\section{Cleavage}

The peptide was cleaved from the resin with $20 \%(\mathrm{v} / \mathrm{v})$ hexafluoroisopropanol (HFIP) in $\mathrm{CH}_{2} \mathrm{Cl}_{2}$ for about 30 min, causing the resin to turn a deep red color. The liquid was collected into a flask, and the resin was rinsed with $\mathrm{CH}_{2} \mathrm{Cl}_{2}$. This procedure was repeated to ensure complete cleavage. The crude material was purified using Biotage reversed phase chromatography, eluting with a gradient of $30 \% \mathrm{MeOH}$ in $\mathrm{H}_{2} \mathrm{O}$ to $100 \% \mathrm{MeOH}$. Purity was confirmed by UPLC/MS, and the peptide was carried forward upon concentration and drying.

\section{C-terminal Methylation}

The peptide was dissolved in $\mathrm{MeOH}(\sim 5 \mathrm{~mL}, \sim 0.6 \mathrm{M})$ and cooled in an ice bath. Dropwise addition of (trimethylsilyl)diazomethane solution ( $2 \mathrm{M}, \sim 4$ equiv) proceeded until the reaction mixture retained a light yellow color. The reaction was monitored by UPLC/MS to ensure complete conversion. The reaction was stirred at ambient temperature for $\sim 5 \mathrm{~min}$, after which excess (trimethylsilyl)diazomethane was quenched with $\mathrm{AcOH}$ (drops). The reaction mixture was concentrated under reduced pressure and carried forward to the next step.

\section{N-terminal Benzyl Deprotection}

The peptide was placed under an inert atmosphere, to which $\mathrm{Pd} / \mathrm{C}(10 \mathrm{~mol} \%)$ was added. $\mathrm{MeOH}$ $(\sim 5 \mathrm{~mL}, \sim 0.6 \mathrm{M})$ was added. The reaction flask was evacuated and refilled with $\mathrm{H}_{2}(3 \mathrm{x})$ and was subsequently allowed to stir under ambient temperature for $\sim 1 \mathrm{~h}$. The reaction mixture was filtered using a syringe filter and $\mathrm{MeOH}$ to remove $\mathrm{Pd} / \mathrm{C}$ and then concentrated under reduced pressure. The purity was checked by UPLC/MS. If needed, the peptide was purified using Biotage reversed phase chromatography, eluting with a gradient of $30 \% \mathrm{MeOH}$ in $\mathrm{H}_{2} \mathrm{O}$ to $100 \% \mathrm{MeOH}$.

\section{General Procedure B: Solution-Phase Peptide Synthesis}

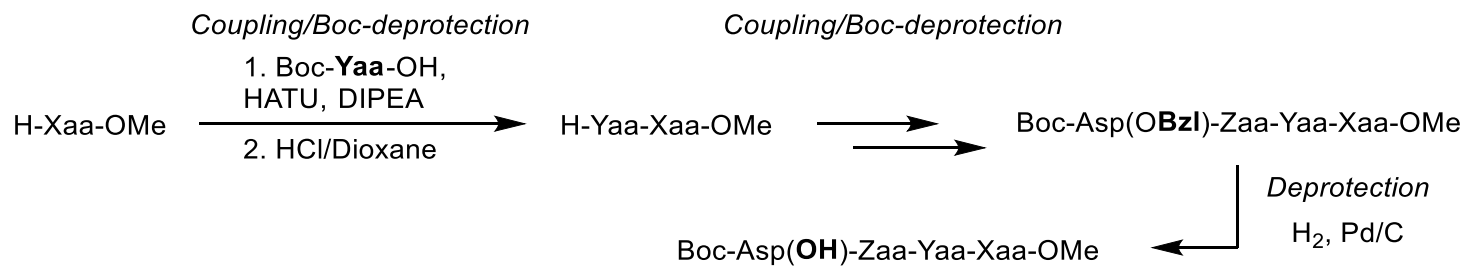

\section{Coupling}

Beginning with the desired $\mathrm{C}$-terminal amino acid methyl ester ( $\mathrm{H}$-Xaa-OMe, 1 equiv), peptide couplings proceeded in $\mathrm{CH}_{2} \mathrm{Cl}_{2}(1 \mathrm{M})$ using the appropriate Boc-protected amino acid (Boc-Yaa$\mathrm{OH}, 1.2$ equiv), HATU (1.2 equiv) and DIPEA (2.4 equiv). The reaction was allowed to stir at ambient temperature for $\sim 30 \mathrm{~min}$ to $1 \mathrm{~h}$. The reaction was monitored by UPLC/MS to ensure complete conversion. Upon completion, the reaction was washed using aqueous citric acid (10\% $\mathrm{w} / \mathrm{v}, 15 \mathrm{~mL})$, saturated $\mathrm{NaHCO}_{3}$ solution $(15 \mathrm{~mL})$, and brine $(15 \mathrm{~mL})$. The aqueous layers were extracted with $\mathrm{CH}_{2} \mathrm{Cl}_{2}(15 \mathrm{~mL})$. The combined organic layers were dried using $\mathrm{Na}_{2} \mathrm{SO}_{4}$ and concentrated under reduced pressure. Coupling and deprotection steps proceeded in sequence until the desired $N$-terminal residue is reached (in most cases, Boc-Asp(OBzl)-OH, 1.2 equiv). The peptide underwent benzyl deprotection using $\mathrm{Pd} / \mathrm{C}$ and $\mathrm{H}_{2}$ as described in the above procedure to reveal the free acid. 


\section{Boc-Deprotection}

Boc-protected amino acids were subjected to $4 \mathrm{M} \mathrm{HCl} /$ dioxane $(\sim 3 \mathrm{~mL}, \sim 1 \mathrm{M})$, stirring at ambient temperature for $\sim 1 \mathrm{~h}$. The reaction was monitored by UPLC/MS to ensure complete conversion. Upon concentration under reduced pressure, $\mathrm{CH}_{2} \mathrm{Cl}_{2}$ was added to the flask, which was concentrated under reduced pressure again to provide white solids, in most cases. Before carrying forward to the next coupling step, the peptide was dried under high vacuum for $\sim 1 \mathrm{~h}$.

\section{Peptide Characterization Data}

\section{Boc-Asp-Pro-D-Lys(Z)-D-Pro-Tyr(t-Bu)-OMe (P1)}

P1 was synthesized according to General Procedure A, and its spectroscopic data matched that in a previous report. ${ }^{1}$

\section{Boc-Asp-Pro-Asn(trt)-D-Phe-Pro-Asn(trt)-OMe (P2)}

P2 was synthesized according to General Procedure A, and its spectroscopic data matched that in a previous report. ${ }^{2}$

Peptides P4-P9, P20, and S1-S5 were synthesized according to General Procedure A, and their spectroscopic data matched that in a previous report. ${ }^{3}$

(S1: Boc-D-Asp-D-Pro-Acpc-Phe-OMe, S2: Boc-D-Asp-Pro-Acpc-Phe-OMe, S3: Boc-AspPro-D-Val-Leu-NH'Pr, S4: CBz-D-Asp-D-Hyp(Bn)-Val-(R)-MBA, S5: Boc-Asp-D-Ser(Bn)-D-ProDap(Boc)-OMe)

\section{Boc-Asp-Acpc-D-Asp(t-Bu)-Val-OMe (P10)}

P10 was synthesized according to General Procedure A using $\mathrm{H}-\mathrm{Val}-2-\mathrm{Cl}-T r t$ resin $(0.85 \mathrm{mmol})$, providing a white solid (371 $\mathrm{mg}, 73 \%$ yield); $\mathbf{R}_{\mathbf{f}} 0.19\left(5 \% \mathrm{MeOH}, \mathrm{CH}_{2} \mathrm{Cl}_{2}\right) ;[\boldsymbol{\alpha}]_{\mathrm{D}}{ }^{20}+19.5(c 0.50$, $\left.\mathrm{CHCl}_{3}\right) ;{ }^{1} \mathrm{H}$ NMR $\left(500 \mathrm{MHz}, \mathrm{CDCl}_{3}\right) \delta 7.64(\mathrm{~d}, J=8.9 \mathrm{~Hz}, 1 \mathrm{H}), 7.24(\mathrm{br} \mathrm{s}, 1 \mathrm{H}), 7.05$ (d, J = $7.5 \mathrm{~Hz}$, $1 \mathrm{H}), 5.70(\mathrm{~d}, J=9.6 \mathrm{~Hz}, 1 \mathrm{H}), 4.90(\mathrm{td}, J=8.2,5.7 \mathrm{~Hz}, 1 \mathrm{H}), 4.52(\mathrm{~s}, 1 \mathrm{H}), 4.20(\mathrm{t}, J=7.1 \mathrm{~Hz}, 1 \mathrm{H})$, $3.74(\mathrm{~s}, 3 \mathrm{H}), 3.22(\mathrm{~d}, J=17.5 \mathrm{~Hz}, 1 \mathrm{H}), 2.86(\mathrm{dd}, J=16.0,5.7 \mathrm{~Hz}, 1 \mathrm{H}), 2.69(\mathrm{dd}, J=17.6,4.2 \mathrm{~Hz}$, 2H), $2.23-2.08(\mathrm{~m}, J=6.8 \mathrm{~Hz}, 1 \mathrm{H}), 1.77-1.64(\mathrm{~m}, 1 \mathrm{H}), 1.53-1.48(\mathrm{~m}, 1 \mathrm{H}), 1.46(\mathrm{~s}, 9 \mathrm{H}), 1.42$ $(\mathrm{s}, 9 \mathrm{H}), 1.11-1.03(\mathrm{~m}, 2 \mathrm{H}), 0.97(\mathrm{~d}, J=6.8 \mathrm{~Hz}, 3 \mathrm{H}), 0.93(\mathrm{~d}, J=6.8 \mathrm{~Hz}, 3 \mathrm{H}) ;{ }^{13} \mathrm{C}$ NMR $(126$ $\left.\mathrm{MHz}_{,} \mathrm{CDCl}_{3}\right) \delta 174.8,173.8,173.1,171.31,171.25,170.8,155.6,81.6,81.2,58.7,52.4,51.1$, 50.3, 37.2, 36.7, 34.8, 30.3, 28.3, 28.0, 19.0, 18.4, 17.9, 16.8; IR (neat) 3320, 2977, 1718, 1660, 1523, 1368, 1276, 1251, 1211, 1152, 1045, 1025, 915, 848, 730, $647 \mathrm{~cm}^{-1}$; HRMS (ESI) m/z calc'd for $\mathrm{C}_{27} \mathrm{H}_{45} \mathrm{~N}_{4} \mathrm{O}_{11}\left[\mathrm{M}+\mathrm{H}^{+}\right]$601.3085, found 601.3102.

\section{Boc-Asp-D-Phe-D-Asp(t-Bu)-Val-OMe (P11)}

P11 was synthesized according to General Procedure A using H-Val-2-Cl-Trt resin $(0.29 \mathrm{mmol})$, providing a white solid (50 mg, 26\% yield); HRMS (ESI) m/z calc'd for $\mathrm{C}_{32} \mathrm{H}_{49} \mathrm{~N}_{4} \mathrm{O}_{11}\left[\mathrm{M}+\mathrm{H}^{+}\right]$ 665.3398 , found 665.3413.

\section{Boc-Asp-D-Val-D-Asp(t-Bu)-Val-OMe (P12)}

P12 was synthesized according to General Procedure A using H-Val-2-Cl-Trt resin $(0.29 \mathrm{mmol})$, providing a white solid (97 mg, 55\% yield); HRMS (ESI) m/z calc'd for $\mathrm{C}_{28} \mathrm{H}_{49} \mathrm{~N}_{4} \mathrm{O}_{11}\left[\mathrm{M}_{+} \mathrm{H}^{+}\right]$ 617.3398 , found 617.3398 .

Boc-Asp-Phe-D-Asp(t-Bu)-Val-OMe (P13) 
P13 was synthesized according to General Procedure A using H-Val-2-Cl-Trt resin $(0.29 \mathrm{mmol})$, providing a white solid (122 mg, 64\% yield); HRMS (ESI) m/z calc'd for $\mathrm{C}_{32} \mathrm{H}_{49} \mathrm{~N}_{4} \mathrm{O}_{11}\left[\mathrm{M}+\mathrm{H}^{+}\right]$ 665.3398 , found 665.3390 .

\section{Boc-Asp-Val-D-Asp(t-Bu)-Val-OMe (P14)}

P14 was synthesized according to General Procedure A using H-Val-2-Cl-Trt resin $(0.29 \mathrm{mmol})$, providing a white solid (96 mg, 55\% yield); HRMS (ESI) $\mathrm{m} / \mathrm{z}$ calc'd for $\mathrm{C}_{28} \mathrm{H}_{49} \mathrm{~N}_{4} \mathrm{O}_{11}\left[\mathrm{M}+\mathrm{H}^{+}\right]$ 617.3398 , found 617.3398 .

\section{Boc-Asp-Pro-D-Asp(t-Bu)-Val-OMe (P15)}

P15 was synthesized according to General Procedure A using H-Val-2-Cl-Trt resin $(0.29 \mathrm{mmol})$, providing a white solid (101 mg, 57\% yield) HRMS (ESI) m/z calc'd for $\mathrm{C}_{28} \mathrm{H}_{47} \mathrm{~N}_{4} \mathrm{O}_{11}\left[\mathrm{M}+\mathrm{H}^{+}\right]$ 615.3242 , found 615.3262 .

\section{Boc-Asp-Aic-D-Asn(trt)-Val-OMe (P16)}

P16 was synthesized according to General Procedure A using H-Val-2-Cl-Trt resin $(0.29 \mathrm{mmol})$, providing a white solid (87 mg, $35 \%$ yield) HRMS (ESI) m/z: $\left[\mathrm{M}+\mathrm{H}^{+}\right]$calcd for $\mathrm{C}_{48} \mathrm{H}_{56} \mathrm{~N}_{5} \mathrm{O}_{10}$ 862.4027, found 862.3950 .

\section{Boc-Asp-Aic-D-Leu-Val-OMe (P17)}

P17 was synthesized according to General Procedure A using $\mathrm{H}-\mathrm{Val}-2-\mathrm{Cl}-\mathrm{Trt}$ resin $(0.29 \mathrm{mmol})$, providing a white solid (96 mg, 54\% yield); HRMS (ESI) m/z: $\left[\mathrm{M}+\mathrm{H}^{+}\right]$calcd for $\mathrm{C}_{31} \mathrm{H}_{47} \mathrm{~N}_{4} \mathrm{O}_{9}$ 619.3343 , found 619.3330 .

\section{Boc-Asp-Aic-D-Asp(t-Bu)-Gly-OMe (P18)}

P18 was synthesized according to General Procedure A using H-Gly-2-Cl-Trt resin $(0.25 \mathrm{mmol})$, providing a white solid (31 mg, 19\% yield); HRMS (ESI) m/z calc'd for $\mathrm{C}_{30} \mathrm{H}_{43} \mathrm{~N}_{4} \mathrm{O}_{11}\left[\mathrm{M}+\mathrm{H}^{+}\right]$ 635.2928 , found 635.2927.

\section{Boc-Asp-Aic-D-Asp(t-Bu)-Tyr(t-Bu)-OMe (P19)}

P19 was synthesized according to General Procedure A using H-Val-2-Cl-Trt resin $(0.21 \mathrm{mmol})$, providing a white solid (18 mg, 7\% yield); HRMS (ESI) m/z calc'd for $\mathrm{C}_{41} \mathrm{H}_{57} \mathrm{~N}_{4} \mathrm{O}_{12}\left[\mathrm{M}+\mathrm{H}^{+}\right]$ 797.3973, found 797.3958 .

Boc-Asp-Acpc-D-Asp(OMe)-OMe was synthesized using General Procedure B starting from Boc-D-Asp(OH)-OMe (100 mg, $0.4 \mathrm{mmol}, 1$ equiv), which was first methylated using (trimethylsilyl)diazomethane as described in the methylation protocol in General Procedure A to provide Boc-D-Asp $(\mathrm{OMe})-\mathrm{OMe}$. Then, Boc-group deprotections and peptide couplings proceeded in sequence with Boc-Acpc-OH, followed by Boc-Asp(OBzl)-OH. Benzyl deprotection was performed as outlined in General Procedure A. Upon reversed-phase column purification, a white solid was obtained (111 mg, $60 \%$ yield); HRMS (ESI) m/z calc'd for $\mathrm{C}_{19} \mathrm{H}_{30} \mathrm{~N}_{3} \mathrm{O}_{10}\left[\mathrm{M}+\mathrm{H}^{+}\right]$ 460.1931 , found 460.1927 .

Boc-Asp-Acpc-OMe was synthesized using General Procedure B starting from Boc-Acpc-OH (100 mg, $0.5 \mathrm{mmol}, 1$ equiv), which was first methylated using (trimethylsilyl)diazomethane as 
described in the methylation protocol in General Procedure A to provide Boc-Acpc-OMe. Then, Boc-group deprotection and peptide coupling proceeded with Boc-Asp(OBzl)-OH. Benzyl deprotection was performed as outlined in General Procedure A. Upon reversed-phase column purification, a white semi-solid was obtained (42 mg, 25\% yield); HRMS (ESI) m/z calc'd for $\mathrm{C}_{14} \mathrm{H}_{23} \mathrm{~N}_{2} \mathrm{O}_{7}\left[\mathrm{M}+\mathrm{H}^{+}\right]$331.1505, found 331.1486.

Boc-Asp-Pro-D-Lys(Z)-D-Pro-OMe was synthesized using General Procedure B starting H-DPro-OMe.HCl. Then, Boc-group deprotections and peptide couplings proceeded in sequence with Boc-D-Lys(Z)-OH, Boc-Pro-OH, and Boc-Asp(OBzl)-OH. Benzyl deprotection was performed as outlined in General Procedure A. Upon reversed-phase column purification, a white semi-solid was obtained (203 mg, 29\% yield); HRMS (ESI) m/z calc'd for $\mathrm{C}_{34} \mathrm{H}_{50} \mathrm{~N}_{5} \mathrm{O}_{11}\left[\mathrm{M}_{+} \mathrm{H}^{+}\right]$704.3507, found 704.3506 .

Boc-Asp-Pro-D-Lys(Z)-OMe was synthesized using General Procedure B starting from H-DLys(Z)-OMe.HCl (331 mg, $1 \mathrm{mmol}, 1$ equiv). Then, Boc-group deprotections and peptide couplings proceeded in sequence with Boc-Pro-OH and Boc-Asp(OBzl)-OH. Benzyl deprotection was performed as outlined in General Procedure A. Upon reversed-phase column purification, a white semi-solid was obtained (45 mg, 7\% yield); $\mathbf{H R M S}$ (ESI) m/z calc'd for $\mathrm{C}_{29} \mathrm{H}_{43} \mathrm{~N}_{4} \mathrm{O}_{10}\left[\mathrm{M}+\mathrm{H}^{+}\right]$ 607.2979 , found 607.2968 .

Boc-Asp-Pro-OMe was synthesized using General Procedure B starting from $\mathrm{H}-\mathrm{Pro}-\mathrm{OMe} . \mathrm{HCl}$ (166 mg, $1 \mathrm{mmol}, 1$ equiv). Benzyl deprotection was performed as outlined in General Procedure A. Upon reversed-phase column purification, a white semi-solid was obtained (94 $\mathrm{mg}, 27 \%$ yield); HRMS (ESI) m/z calc'd for $\mathrm{C}_{15} \mathrm{H}_{25} \mathrm{~N}_{2} \mathrm{O}_{7}\left[\mathrm{M}+\mathrm{H}^{+}\right]$345.1662, found 345.1642.

Boc-Asp-D-Pro-OMe was synthesized using General Procedure B starting from H-D-ProOMe. $\mathrm{HCl}$ (99 mg, $0.6 \mathrm{mmol}, 1$ equiv). Benzyl deprotection was performed as outlined in General Procedure A. Upon reversed-phase column purification, a white semi-solid was obtained (108 mg, 63\% yield); HRMS (ESI) m/z calc'd for $\mathrm{C}_{15} \mathrm{H}_{25} \mathrm{~N}_{2} \mathrm{O}_{7}\left[\mathrm{M}+\mathrm{H}^{+}\right]$345.1662, found 345.1661.

Boc-D-Asp-Pro-OMe was synthesized using General Procedure B starting from H-Pro-OMe.HCl (99 mg, $0.6 \mathrm{mmol}, 1$ equiv). Benzyl deprotection was performed as outlined in General Procedure A. Upon reversed-phase column purification, a white semi-solid was obtained (88 $\mathrm{mg}$, $51 \%$ yield); HRMS (ESI) m/z calc'd for $\mathrm{C}_{15} \mathrm{H}_{25} \mathrm{~N}_{2} \mathrm{O}_{7}\left[\mathrm{M}+\mathrm{H}^{+}\right]$345.1662, found 345.1656.

Boc-D-Asp-D-Pro-OMe was synthesized using General Procedure B starting from H-D-ProOMe. $\mathrm{HCl}$ (99 mg, $0.6 \mathrm{mmol}, 1$ equiv). Benzyl deprotection was performed as outlined in General Procedure A. Upon reversed-phase column purification, a white semi-solid was obtained (75 mg, $44 \%$ yield); HRMS (ESI) m/z calc'd for $\mathrm{C}_{15} \mathrm{H}_{25} \mathrm{~N}_{2} \mathrm{O}_{7}\left[\mathrm{M}+\mathrm{H}^{+}\right] 345.1662$, found 345.1660. 


\section{Peptide Screening Conditions and Results}

\section{General Procedure C: Small Scale Peptide Screening}

Several reactions were performed simultaneously. The appropriate peptides (50 mol\%) were added to individual 1 dram vials equipped with stir bars. Standard solutions of i) geldanamycin ( $2.8 \mathrm{mg}$ or $5 \mu \mathrm{mol}$ per reaction, 1 equiv), including benzyl benzoate $(1.5 \mu \mathrm{L}$ per reaction) as an internal standard, and ii) DMAP (0.3 mg or $2.5 \mu \mathrm{mol}$ per reaction, $50 \mathrm{~mol} \%$ ) were prepared in $\mathrm{CHCl}_{3}$, so that the final volume totaled $0.5 \mathrm{~mL} \mathrm{CHCl} 3(10 \mathrm{mM})$ per reaction. The appropriate amount of each standard solution was added to the reaction vials, to which were added $\mathrm{H}_{2} \mathrm{O}_{2}$ ( $30 \%$ aq, $2.5 \mu \mathrm{L}, 25 \mu \mathrm{mol}, 5$ equiv), followed by DIC ( $3.9 \mu \mathrm{L}, 25 \mu \mathrm{mol}, 5$ equiv). The reactions were stirred vigorously at ambient temperature for $24 \mathrm{~h}$, after which they were filtered through a short plug of $\mathrm{SiO}_{2}$ and $\mathrm{Na}_{2} \mathrm{SO}_{4}$ with $10 \% \mathrm{MeOH} / \mathrm{CH}_{2} \mathrm{Cl}_{2}$ and concentrated under reduced pressure. The reactions were prepared for analysis by UPLC/MS.

\section{General Procedure D: Reactions with $m$-CPBA}

A standard solution of geldanamycin (2.8 $\mathrm{mg}$ or $5 \mu \mathrm{mol}$ per reaction, 1 equiv), including benzyl benzoate $\left(1.5 \mu \mathrm{L}\right.$ per reaction) as an internal standard, in $\mathrm{CH}_{2} \mathrm{Cl}_{2}$ was prepared so that the final reaction volume totaled $0.5 \mathrm{~mL} \mathrm{CH} \mathrm{Cl}_{2}(10 \mathrm{mM})$. To this solution in a vial equipped with stir bar was added $m$-CPBA ( 2 equiv). The reactions were stirred at ambient temperature for $24 \mathrm{~h}$, after which they were filtered through a short plug of $\mathrm{SiO}_{2}$ and $\mathrm{Na}_{2} \mathrm{SO}_{4}$ with $10 \% \mathrm{MeOH} / \mathrm{CH}_{2} \mathrm{Cl}_{2}$ and concentrated under reduced pressure. The reactions were prepared for analysis by UPLC/MS. 
Table S1. Results from Peptide Screening (Figures 1 and 2) ${ }^{\mathrm{a}}$

\begin{tabular}{|c|c|c|c|c|c|c|}
\hline Entry & Peptide & $\% A^{b, c}$ & $\% \mathrm{~B}^{\mathrm{b}, \mathrm{c}}$ & $\% \mathbf{C}^{b, c}$ & $\% D^{b, c}$ & $\%$ Yield $^{d}$ \\
\hline 1.2 & Boc-Asp-OMe & 7.0 & 14.3 & 7.3 & 0 & 28.6 \\
\hline 1.3 & P4 & 11.3 & 6.7 & 6.2 & 0 & 24.2 \\
\hline 1.4 & P5 & 10.8 & 11.7 & 19.0 & 2.5 & 43.9 \\
\hline 1.5 & P6 & 5.4 & 9.4 & 3.6 & 0.0 & 18.4 \\
\hline 1.6 & P7 & 6.1 & 8.4 & 4.4 & 6.9 & 25.8 \\
\hline 1.7 & P2 & 1.4 & 2.5 & 3.9 & 0.5 & 8.5 \\
\hline 1.8 & P8 & 59.1 & 22.2 & 4.8 & 1.0 & 87.1 \\
\hline 1.9 & P9 & 45.5 & 19.3 & 2.8 & 0.3 & 67.9 \\
\hline 1.10 & P1 & 0.3 & 0.6 & 26.6 & 7.5 & 35.0 \\
\hline 2.1 & P10 & 19.0 & 6.1 & 4.2 & 1.6 & 30.9 \\
\hline 2.2 & P11 & 25.3 & 11.2 & 3.1 & 4.5 & 44.1 \\
\hline 2.3 & P12 & 29.8 & 12.3 & 5.6 & 2.9 & 50.7 \\
\hline 2.4 & P13 & 23.4 & 10.5 & 6.3 & 4.7 & 45.0 \\
\hline 2.5 & P14 & 13.3 & 10.5 & 7.7 & 0.6 & 32.0 \\
\hline 2.6 & P15 & 6.7 & 6.8 & 5.7 & 0 & 19.2 \\
\hline 2.7 & P16 & 12.0 & 17.4 & 3.3 & 1.5 & 34.2 \\
\hline 2.8 & P17 & 14.2 & 6.3 & 4.9 & 4.9 & 30.3 \\
\hline 2.9 & P18 & 31.5 & 24.1 & 3.9 & 1.9 & 61.4 \\
\hline 2.10 & P19 & 41.3 & 18.6 & 4.2 & 3.6 & 67.7 \\
\hline 2.11 & P20 & 24.1 & 13.9 & 20.4 & 2.4 & 60.9 \\
\hline & S1 & 8.7 & 5.5 & 5.6 & 0 & 19.8 \\
\hline & S2 & 11.4 & 17.5 & 12.5 & 1.2 & 42.6 \\
\hline & S3 & 5.9 & 8.0 & 7.8 & 6.0 & 27.8 \\
\hline & S4 & 8.2 & 8.3 & 7.6 & 0.7 & 24.7 \\
\hline & S5 & 26.3 & 15.8 & 1.9 & 0.1 & 44.1 \\
\hline
\end{tabular}

${ }^{a}$ All data is an average of at least two trials using General Procedure C. ${ }^{b}$ Determined using UPLC with an internal standard and calibration curve. cProduct distributions shown in main text are adjusted to total $100 \%$. For example $\% A=A /(A+B+C+D) \times 100$. dSummed yields of $A+B+C+D$.

Table S2. Results from Peptide Truncations and Diastereomers (Figure 3) ${ }^{\mathrm{a}}$

\begin{tabular}{|c|c|c|c|c|c|c|}
\hline Entry & Peptide & $\% A^{b, c}$ & $\% B^{b, c}$ & $\% \mathbf{C}^{\mathbf{b}, \mathbf{c}}$ & $\% D^{b, c}$ & $\%$ Yield $^{d}$ \\
\hline 3.1 & $\begin{array}{l}\text { Boc-Asp-Acpc-D- } \\
\text { Asp(OMe)-OMe }\end{array}$ & 18.8 & 9.9 & 5.0 & 0.2 & 33.9 \\
\hline 3.2 & Boc-Asp-Acpc-OMe & 9.7 & 11.7 & 6.9 & 0.6 & 29.0 \\
\hline 3.3 & $\begin{array}{l}\text { Boc-Asp-Pro-D-Lys(Z)-D- } \\
\text { Pro-OMe }\end{array}$ & 0.6 & 0.7 & 4.8 & 0.4 & 6.4 \\
\hline 3.4 & $\begin{array}{c}\text { Boc-Asp-Pro-D-Lys(Z)- } \\
\text { OMe }\end{array}$ & 0.9 & 1.1 & 4.6 & 0.3 & 7.0 \\
\hline 3.5 & Boc-Asp-Pro-OMe & 0.3 & 1.5 & 36.0 & 14.3 & 52.0 \\
\hline 3.6 & Boc-Asp-D-Pro-OMe & 6.7 & 14.2 & 7.7 & 0.3 & 28.8 \\
\hline 3.7 & Boc-D-Asp-Pro-OMe & 7.3 & 14.5 & 5.9 & 0.3 & 28.1 \\
\hline 3.8 & Boc-D-Asp-D-Pro-OMe & 9.7 & 14.7 & 6.0 & 0.3 & 30.6 \\
\hline \multicolumn{7}{|c|}{ 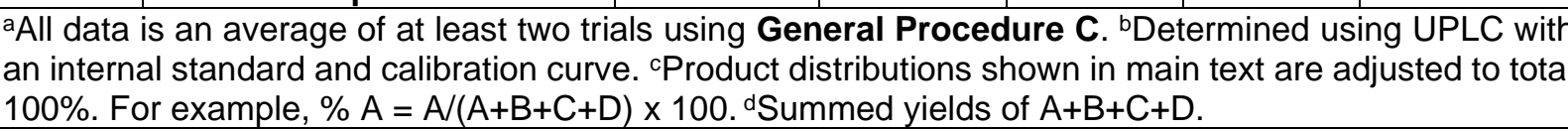 } \\
\hline
\end{tabular}




\section{Large Scale Reaction Conditions}

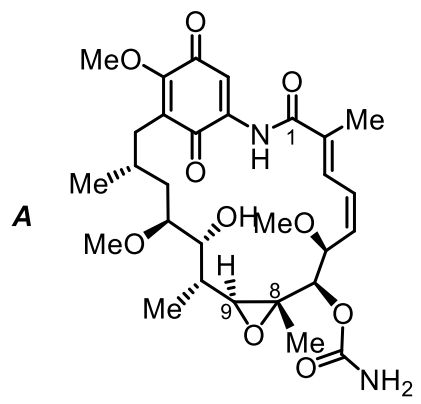

Geldanamycin (14 mg, $25 \mu \mathrm{mol}, 1.0$ equiv), Boc-Asp-Acpc- ${ }^{\mathrm{D}} \mathrm{Asp}(t-\mathrm{Bu})$ Val-OMe (3.8 mg, $6.3 \mu \mathrm{mol}, 25 \mathrm{~mol} \%$ ), and DMAP (0.80 mg, $6.3 \mu \mathrm{mol}$, $25 \mathrm{~mol} \%$ ) are added to a $10 \mathrm{~mL}$ round bottom flask, equipped with a stir bar. Chloroform (2.5 mL) is added to the flask, followed by $\mathrm{H}_{2} \mathrm{O}_{2}$ (30\% aq. soln, $25 \mu \mathrm{L}, 250 \mu \mathrm{mol}, 10$ equiv) and DIC (39 $\mu \mathrm{L}, 250 \mu \mathrm{mol}$, 10 equiv). The reaction is stirred vigorously for $24 \mathrm{~h}$ at ambient temperature. The reaction is filtered through a short plug of $\mathrm{Na}_{2} \mathrm{SO}_{4}$ and silica using $\mathrm{MeOH} / \mathrm{CH}_{2} \mathrm{Cl}_{2}(10 \% \mathrm{v} / \mathrm{v})$. An internal standard is added (benzyl benzoate), and the reaction is analyzed using UPLC. The reaction mixture is purified using reversed phase preparatory HPLC, followed by extraction with $\mathrm{CH}_{2} \mathrm{Cl}_{2}$, to provide $\mathbf{A}$ as a yellow solid (5.6 $\mathrm{mg}, 38 \%$ yield): $\mathbf{R}_{\boldsymbol{f}}$ $0.46\left(10 \% \mathrm{MeOH}\right.$ in $\left.\mathrm{CH}_{2} \mathrm{Cl}_{2}\right)$; $[\alpha]_{D}{ }^{20}+125.6$ (c $\left.0.20,10 \% \mathrm{MeOH} / \mathrm{CHCl}_{3}\right) ;{ }^{1} \mathbf{H}$ NMR $(500 \mathrm{MHz}$, $\left.\mathrm{CDCl}_{3}\right) \delta 8.84(\mathrm{~s}, 1 \mathrm{H}), 7.22(\mathrm{~s}, 1 \mathrm{H}), 6.94(\mathrm{~s}, 1 \mathrm{H}), 6.53(\mathrm{t}, J=11.4 \mathrm{~Hz}, 1 \mathrm{H}), 5.89-5.75(\mathrm{~m}, 1 \mathrm{H})$, $4.95(\mathrm{~s}, 1 \mathrm{H}), 4.77(\mathrm{br} \mathrm{s}, 2 \mathrm{H}) 4.53(\mathrm{~s}, 1 \mathrm{H}), 4.12(\mathrm{~s}, 3 \mathrm{H}), 3.81(\mathrm{dd}, J=7.5,2.2 \mathrm{~Hz}, 1 \mathrm{H}), 3.45-3.40$ $(\mathrm{m}, 1 \mathrm{H}), 3.40(\mathrm{~s}, 3 \mathrm{H}), 3.33(\mathrm{~s}, 3 \mathrm{H}), 2.96(\mathrm{br} \mathrm{s}, 1 \mathrm{H}), 2.51-2.37(\mathrm{~m}, 2 \mathrm{H}), 2.01(\mathrm{~s}, 3 \mathrm{H}), 1.90-1.81$ $(\mathrm{m}, 1 \mathrm{H}), 1.79-1.72(\mathrm{~m}, 1 \mathrm{H}), 1.70-1.64(\mathrm{~m}, 3 \mathrm{H}), 1.42(\mathrm{~s}, 3 \mathrm{H}), 1.30-1.22(\mathrm{~m}, 2 \mathrm{H}), 1.09(\mathrm{~d}, J=$ $6.8 \mathrm{~Hz}, 3 \mathrm{H}), 0.97(\mathrm{~d}, J=6.5 \mathrm{~Hz}, 3 \mathrm{H}) ;{ }^{13} \mathrm{C}$ NMR $\left(126 \mathrm{MHz}, 10 \% \mathrm{MeOD}-\mathrm{d}_{4} / \mathrm{CDCl}_{3}\right) \delta 184.3,184.2$, 169.0, 157.1, 156.8, 138.2, 135.3, 128.0, 127.6, 126.1, 111.2, 111.2, 81.2, 76.5, 70.8, 62.8, 61.8, 61.5, 57.1, 56.9, 34.0, 32.8, 31.7, 29.6, 29.2, 22.2, 14.4, 12.2, 10.3; IR (neat) 3435, 2924, 2853, 1739, 1708, 1697, 1653, 1612, 1514, 1458, 1378, 1359, 1201, 1107, 1064, $1043 \mathrm{~cm}^{-1}$; HRMS (ESI) $\mathrm{m} / \mathrm{z}$ calc'd for $\mathrm{C}_{29} \mathrm{H}_{41} \mathrm{~N}_{2} \mathrm{O}_{10}\left[\mathrm{M}+\mathrm{H}^{+}\right]$577.2761, found 577.2785.

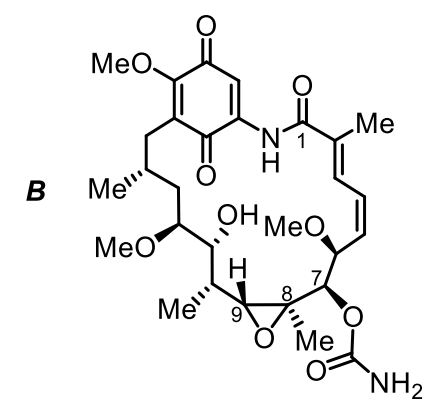

In the same reaction as above, $\mathbf{B}$ was isolated as a yellow semisolid (1.4 mg, $10 \%$ yield): $\mathbf{R}_{f} 0.25\left(5 \% \mathrm{MeOH}\right.$ in $\left.\mathrm{CH}_{2} \mathrm{Cl}_{2}\right) ;[\alpha]_{\mathrm{D}}{ }^{20}+33.3(c 0.04$, $\left.\mathrm{CHCl}_{3}\right) ;{ }^{1} \mathrm{H}$ NMR $\left(500 \mathrm{MHz}, \mathrm{CDCl}_{3}\right) \delta 8.70(\mathrm{~s}, 1 \mathrm{H}), 7.23(\mathrm{~s}, 1 \mathrm{H}), 6.97$ $(\mathrm{d}, J=11.4 \mathrm{~Hz}, 1 \mathrm{H}), 6.49$ (t, $J=11.3 \mathrm{~Hz}, 1 \mathrm{H}), 5.85(\mathrm{t}, J=10.0 \mathrm{~Hz}, 1 \mathrm{H})$, $4.86(\mathrm{br} \mathrm{s}, 2 \mathrm{H}), 4.65(\mathrm{~d}, J=8.9 \mathrm{~Hz}, 1 \mathrm{H}), 4.14-4.09(\mathrm{~m}, 1 \mathrm{H}), 4.09(\mathrm{~s}$, $3 \mathrm{H}), 4.01-3.95(\mathrm{~m}, 1 \mathrm{H}), 3.72-3.64(\mathrm{~m}, 1 \mathrm{H}), 3.37(\mathrm{~s}, 3 \mathrm{H}), 3.33(\mathrm{~s}$, $3 \mathrm{H}), 3.06(\mathrm{~d}, J=8.8 \mathrm{~Hz}, 1 \mathrm{H}), 2.62(\mathrm{~d}, J=12.5 \mathrm{~Hz}, 1 \mathrm{H}), 2.38-2.23(\mathrm{~m}$, $1 \mathrm{H}), 2.00(\mathrm{~s}, 3 \mathrm{H}), 1.72-1.47(\mathrm{~m}, 6 \mathrm{H}), 1.39(\mathrm{~s}, 3 \mathrm{H}), 1.03(\mathrm{~d}, J=6.8 \mathrm{~Hz}$, $3 \mathrm{H}), 0.91(\mathrm{~d}, J=6.5 \mathrm{~Hz}, 3 \mathrm{H}) ;{ }^{13} \mathrm{C}$ NMR $\left(151 \mathrm{MHz}, \mathrm{CDCl}_{3}\right) \delta 184.3$, 184.1, 168.7, 163.1, 156.7, 156.1, 138.5, 136.7, 135.2, 128.5, 127.5, 126.2, 116.4, 111.5, 81.7, 81.3, 75.8, 72.6, 61.5, 60.4, 57.1, 56.2, 36.5, 34.7, 29.7, 22.1, 12.5, 12.3, 11.3; IR (neat) 2953, 2925, 2854, 2181, 2160, 2154, 2044, 2027, 1723, 1652, 1605, 1501, 1376, 1193, 1135, $1072 \mathrm{~cm}^{-1}$; HRMS (ESI) m/z calc'd for $\mathrm{C}_{29} \mathrm{H}_{41} \mathrm{~N}_{2} \mathrm{O}_{10}\left[\mathrm{M}+\mathrm{H}^{+}\right]$577.2761, found 577.2740 .

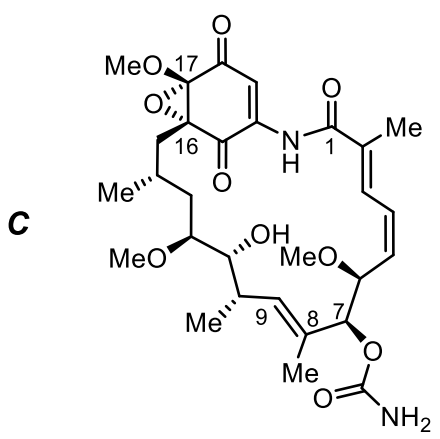

Geldanamycin (14 mg, $25 \mu \mathrm{mol}, 1.0$ equiv), Boc-Asp-Pro-'Lys(Z)DPro-Tyr(t-Bu)-OMe (3.8 mg, $6.3 \mu \mathrm{mol}, 25 \mathrm{~mol} \%)$, and DMAP (3.2 $\mathrm{mg}, 25 \mu \mathrm{mol}, 1$ equiv) are added to a $10 \mathrm{~mL}$ round bottom flask, equipped with a stir bar. Chloroform $(2.5 \mathrm{~mL})$ is added to the flask, followed by $\mathrm{H}_{2} \mathrm{O}_{2}$ ( $30 \%$ aq. soln, $50 \mu \mathrm{L}, 5 \mathrm{mmol}, 20$ equiv) and $\mathrm{DIC}$ (78 $\mu \mathrm{L}, 5 \mathrm{mmol}, 20$ equiv). The reaction is stirred vigorously for 24 $\mathrm{h}$ at ambient temperature. The reaction is filtered through a short plug of $\mathrm{Na}_{2} \mathrm{SO}_{4}$ and silica using $\mathrm{MeOH} / \mathrm{CH}_{2} \mathrm{Cl}_{2}(10 \% \mathrm{v} / \mathrm{v})$. An internal standard is added (benzyl benzoate), and the reaction is analyzed using UPLC. The reaction mixture is purified using reversed phase preparatory HPLC, followed by extraction with $\mathrm{CH}_{2} \mathrm{Cl}_{2}$, to provide $\mathbf{C}$ as an off-white solid (4.7 mg, 
$33 \%$ yield): $\mathbf{R}_{\boldsymbol{f}} 0.45\left(10 \% \mathrm{MeOH}\right.$ in $\left.\mathrm{CH}_{2} \mathrm{Cl}_{2}\right) ;[\boldsymbol{\alpha}]_{\mathrm{D}}{ }^{20}+143.3$ (c $\left.0.24,10 \% \mathrm{MeOH} / \mathrm{CHCl}_{3}\right) ;{ }^{1} \mathbf{H ~ N M R}$ $\left(500 \mathrm{MHz}, \mathrm{CDCl}_{3}\right) \delta 8.42(\mathrm{~s}, 1 \mathrm{H}), 7.51(\mathrm{~s}, 1 \mathrm{H}), 6.91(\mathrm{~d}, J=10.5 \mathrm{~Hz}, 1 \mathrm{H}), 6.54(\mathrm{t}, J=11.8 \mathrm{~Hz}, 1 \mathrm{H})$, $5.89(\mathrm{t}, J=10.2 \mathrm{~Hz} 1 \mathrm{H}), 5.69(\mathrm{~d}, J=9.5 \mathrm{~Hz}, 1 \mathrm{H}), 5.24(\mathrm{~s}, 1 \mathrm{H}), 4.73(\mathrm{br} \mathrm{s}, 2 \mathrm{H}), 4.33(\mathrm{~d}, J=8.6 \mathrm{~Hz}$, $1 \mathrm{H}), 3.78(\mathrm{~s}, 3 \mathrm{H}), 3.52(\mathrm{dd}, J=8.1,3.6 \mathrm{~Hz}, 1 \mathrm{H}), 3.41-3.38(\mathrm{~m}, 1 \mathrm{H}), 3.37(\mathrm{~s}, 3 \mathrm{H}), 3.32(\mathrm{~s}, 3 \mathrm{H})$, $2.76(\mathrm{tt}, \mathrm{J}=10.1,6.8 \mathrm{~Hz}, 1 \mathrm{H}), 2.37(\mathrm{dd}, \mathrm{J}=14.0,4.3 \mathrm{~Hz}, 1 \mathrm{H}), 2.01(\mathrm{~s}, 3 \mathrm{H}), 1.96-1.88(\mathrm{~m}, 1 \mathrm{H})$, $1.84-1.74(\mathrm{~m}, 4 \mathrm{H}), 1.77(\mathrm{~s}, 3 \mathrm{H}), 1.00(\mathrm{t}, \mathrm{J}=6.4 \mathrm{~Hz}, 6 \mathrm{H}) ;{ }^{13} \mathrm{C}$ NMR $(126 \mathrm{MHz}, 10 \% \mathrm{MeOD}-$ $\left.\mathrm{d}_{4} / \mathrm{CDCl}_{3}\right) \delta 190.5,189.2,168.9,156.8,139.6,136.7,134.4,132.8,132.1,128.2,126.2,114.1$, $84.1,81.4,80.9,80.7,73.2,67.3,57.4,57.2,56.7,34.8,33.2,32.8,25.7,23.0,13.3,13.2,12.4$; IR (neat) 3436, 2925, 2854, 1735, 1684, 1616, 1514, 1379, 1349, 1328, 1132, 1109, $1049 \mathrm{~cm}^{-1}$; HRMS (ESI) $\mathrm{m} / \mathrm{z}$ calc'd for $\mathrm{C}_{29} \mathrm{H}_{41} \mathrm{~N}_{2} \mathrm{O}_{10}\left[\mathrm{M}+\mathrm{H}^{+}\right]$577.2761, found 577.2736.

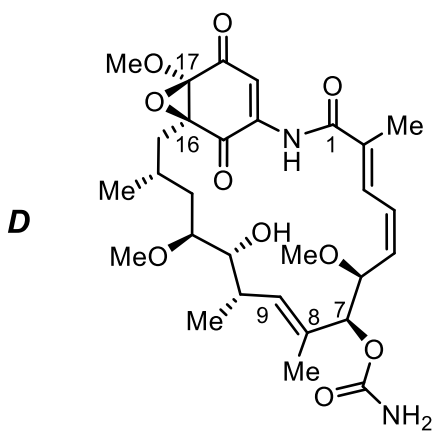

In the same reaction as above, $\mathbf{D}$ was isolated as an off-white solid $(2.0 \mathrm{mg}, 14 \%$ yield $): \mathbf{R}_{\boldsymbol{f}} 0.56\left(10 \% \mathrm{MeOH}\right.$ in $\left.\mathrm{CH}_{2} \mathrm{Cl}_{2}\right) ;[\alpha]_{\mathrm{D}}{ }^{20}+13.9(\mathrm{c}$ $\left.0.18, \mathrm{CHCl}_{3}\right) ;{ }^{1} \mathrm{H}$ NMR $\left(500 \mathrm{MHz}, \mathrm{CDCl}_{3}\right) \delta 8.41(\mathrm{~s}, 1 \mathrm{H}), 7.43(\mathrm{~s}, 1 \mathrm{H})$, $6.86(\mathrm{~d}, J=11.7 \mathrm{~Hz}, 1 \mathrm{H}), 6.52(\mathrm{t}, J=11.3 \mathrm{~Hz}, 1 \mathrm{H}), 5.95(\mathrm{t}, J=9.0 \mathrm{~Hz}$, $1 \mathrm{H}), 5.54(\mathrm{~d}, J=9.8 \mathrm{~Hz}, 1 \mathrm{H}), 5.01(\mathrm{~s}, 1 \mathrm{H}), 4.87(\mathrm{~s}, 2 \mathrm{H}), 4.30(\mathrm{~d}, \mathrm{~J}=$ $8.1 \mathrm{~Hz}, 1 \mathrm{H}), 3.78(\mathrm{~s}, 3 \mathrm{H}), 3.39(\mathrm{~s}, 3 \mathrm{H}), 3.34(\mathrm{~s}, 3 \mathrm{H}), 3.31(\mathrm{~d}, \mathrm{~J}=10.3$ $\mathrm{Hz}, 1 \mathrm{H}), 3.24(\mathrm{~d}, J=9.1 \mathrm{~Hz}, 1 \mathrm{H}), 2.90-2.76(\mathrm{~m}, 1 \mathrm{H}), 2.24-2.10(\mathrm{~m}$, $2 \mathrm{H}), 2.00(\mathrm{~s}, 3 \mathrm{H}), 1.90-1.82(\mathrm{~m}, 1 \mathrm{H}), 1.77(\mathrm{~s}, 3 \mathrm{H}), 1.71-1.62(\mathrm{~m}$, $2 \mathrm{H}), 1.36-1.28(\mathrm{~m}, 1 \mathrm{H}), 1.10(\mathrm{~d}, J=6.1 \mathrm{~Hz}, 3 \mathrm{H}), 0.84(\mathrm{~d}, J=6.9 \mathrm{~Hz}$, $3 \mathrm{H}) ;{ }^{13} \mathrm{C}$ NMR $\left(126 \mathrm{MHz}, \mathrm{CDCl}_{3}\right) \delta 190.4,188.7,168.2,156.0,139.3$, 138.4, 134.5, 134.0, 131.9, 127.8, 125.8, 113.8, 84.5, 82.6, 82.5, 80.5, 73.0, 67.1, 57.9, 57.2, 56.8, 33.0, 31.9, 31.7, 29.7, 24.3, 12.6, 12.4, 12.0; IR (neat); 3454, 2926, 2855, 1717, 1682, 1502, $1456,1376,1325,1198,1129,1097,1058 \mathrm{~cm}^{-1} ;$ HRMS (ESI) m/z calc'd for $\mathrm{C}_{29} \mathrm{H}_{41} \mathrm{~N}_{2} \mathrm{O}_{10}\left[\mathrm{M}_{+} \mathrm{H}^{+}\right]$ 577.2761, found 577.2770 .

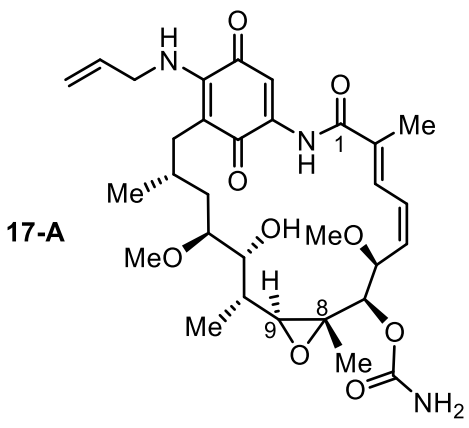

17-AAG (14.6 mg, $25 \mu \mathrm{mol}, 1.0$ equiv), Boc-Asp-Acpc- ${ }^{\mathrm{D}} \mathrm{Asp}(t-\mathrm{Bu})$ Val-OMe (3.8 mg, $6.3 \mu \mathrm{mol}, 25 \mathrm{~mol} \%$ ), and DMAP (0.80 mg, 6.3 umol, $25 \mathrm{~mol} \%$ ) are added to a $10 \mathrm{~mL}$ round bottom flask, equipped with a stir bar. Chloroform $(2.5 \mathrm{~mL})$ is added to the flask, followed by $\mathrm{H}_{2} \mathrm{O}_{2}$ (30\% aq. soln, $25 \mu \mathrm{L}, 250 \mu \mathrm{mol}, 10$ equiv) and DIC (39 $\mu \mathrm{L}, 250 \mu \mathrm{mol}, 10$ equiv). The reaction is stirred vigorously for $24 \mathrm{~h}$ at ambient temperature. The reaction is filtered through a short plug of $\mathrm{Na}_{2} \mathrm{SO}_{4}$ and silica using $\mathrm{MeOH} / \mathrm{CH}_{2} \mathrm{Cl}_{2}(10 \% \mathrm{v} / \mathrm{v})$. An internal standard is added (benzyl benzoate), and the reaction is analyzed using UPLC. The reaction mixture is purified using reversed phase preparatory HPLC, followed by extraction with $\mathrm{CH}_{2} \mathrm{Cl}_{2}$, to provide 17-A as a purple solid (4.9 mg, 32\% yield): $\mathbf{R}_{f} 0.43\left(5 \% \mathrm{MeOH} / \mathrm{CH}_{2} \mathrm{Cl}_{2}\right)$; [a] ${ }_{\mathrm{D}}{ }^{20}-73.2\left(c 0.06, \mathrm{CHCl}_{3}\right) ;{ }^{1} \mathrm{H}$ NMR (500 $\left.\mathrm{MHz}, \mathrm{CDCl}_{3}\right) \delta 9.32(\mathrm{~s}, 1 \mathrm{H}), 7.23(\mathrm{~s}, 1 \mathrm{H}), 6.98(\mathrm{br} \mathrm{d}, J=11.3 \mathrm{~Hz}, 1 \mathrm{H}), 6.58(\mathrm{t}, J=11.4 \mathrm{~Hz}, 1 \mathrm{H})$, $6.43(\mathrm{t}, J=6.0 \mathrm{~Hz}, 1 \mathrm{H}$ ), 5.92 (ddt, $J=16.0,10.5,5.3 \mathrm{~Hz}, 1 \mathrm{H}), 5.80$ (t, $J=9.9 \mathrm{~Hz}, 1 \mathrm{H}$ ), $5.37-5.21$ (m, 2H), $5.16-4.79$ (br s, 2H), $4.74(\mathrm{~s}, 1 \mathrm{H}), 4.49$ (br d, J=9.8 Hz, $1 \mathrm{H}), 4.15$ (t, J = 5.9 Hz, 2H), $3.76(\mathrm{dd}, J=7.8,2.6 \mathrm{~Hz}, 1 \mathrm{H}), 3.45(\mathrm{dt}, J=7.5,3.7 \mathrm{~Hz}, 1 \mathrm{H}), 3.34(\mathrm{~s}, 3 \mathrm{H}), 3.33(\mathrm{~s}, 3 \mathrm{H}) 3.07$ (s, $1 \mathrm{H}), 2.66(\mathrm{~d}, J=13.9 \mathrm{~Hz}, 1 \mathrm{H}), 2.33(\mathrm{dd}, J=14.0,10.2 \mathrm{~Hz}, 1 \mathrm{H}), 2.02(\mathrm{~s}, 3 \mathrm{H}), 1.90-1.76(\mathrm{~m}, 3 \mathrm{H})$, $1.70(\mathrm{t}, J=4.4 \mathrm{~Hz}, 2 \mathrm{H}), 1.45(\mathrm{~s}, 3 \mathrm{H}), 1.11(\mathrm{~d}, J=6.8 \mathrm{~Hz}, 3 \mathrm{H}), 0.99(\mathrm{~d}, J=6.6 \mathrm{~Hz}, 3 \mathrm{H}) ;{ }^{13} \mathrm{C}$ NMR (126 MHz, 10\% MeOD- $\mathrm{d}_{4} / \mathrm{CDCl}_{3}$ ) 183.9, 180.4, 168.8, 156.8, 145.0, 141.2, 134.3, 132.6, 127.8, 
$126.5,118.1,108.1,108.1,81.7,75.9,71.1,63.6,62.2,56.7,56.7,47.5,34.5,33.3,32.9,29.5$, 28.1, 27.7, 22.2, 14.2, 12.1, 10.4; IR (neat) 3425, 3362, 2918, 2850, 1737, 1707, 1694, 1587, 1500, 1377, 1106, 1063, $1041 \mathrm{~cm}^{-1}$; HRMS (ESI) m/z calc'd for $\mathrm{C}_{31} \mathrm{H}_{44} \mathrm{~N}_{3} \mathrm{O}_{9}\left[\mathrm{M}+\mathrm{H}^{+}\right] 602.3077$, found 602.3073 .

In the same reaction as above, 17-B was isolated as a purple solid ( $0.5 \mathrm{mg}, 3 \%$ yield): HRMS (ESI) $\mathrm{m} / \mathrm{z}$ calc'd for $\mathrm{C}_{31} \mathrm{H}_{44} \mathrm{~N}_{3} \mathrm{O}_{9}\left[\mathrm{M}+\mathrm{H}^{+}\right]$602.3077, found 602.3087 .

\section{Liquid Chromatography Chromatograms}

\section{UPLC chromatograms}

UPLC method: Gradient $10 \% \operatorname{MeCN}\left(0.1 \%\right.$ formic acid) in $\mathrm{H}_{2} \mathrm{O}(0.1 \%$ formic acid) to $98 \% \mathrm{MeCN}$ $\left(0.1 \%\right.$ formic acid) in $\mathrm{H}_{2} \mathrm{O}(0.1 \%$ formic acid $)$ over $4 \mathrm{~min}$ at $0.8 \mathrm{~mL} / \mathrm{min}$.

Column: CORTECS C18 (1.6 $\mu \mathrm{m}$ particle size, 3.0 x $50 \mathrm{~mm})$

All UPLC data was processed with MestReNova x64 using PDA total absorbance chromatograms and the default settings for "trace baseline correction". Manuel integration intervention was applied when necessary.

Crude reaction mixture using $\mathbf{P} 10$ :

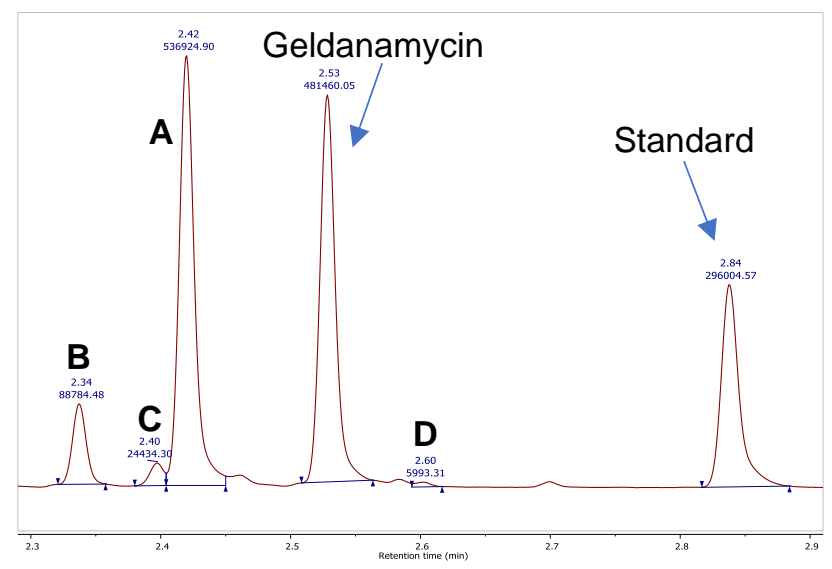



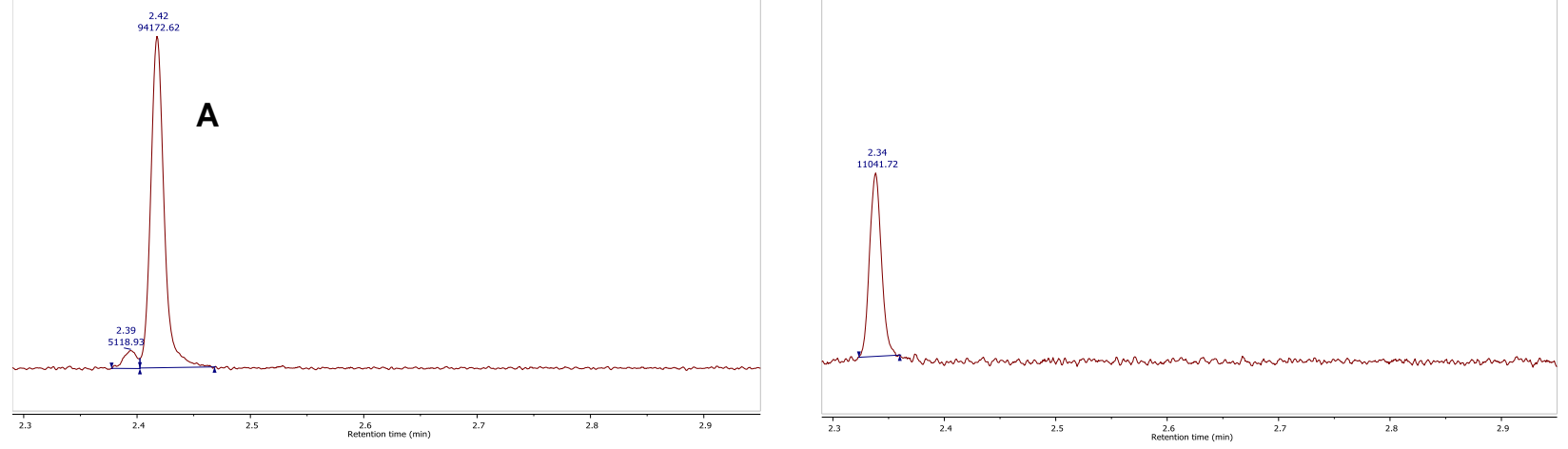

*Small amount of $\mathbf{B}$ remains upon column purification of $\mathbf{A}$. Compound $\mathbf{A}$ can be further purified through crystallization. Via vapor diffusion, $\mathbf{A}$ was dissolved in a minimal amount of $10 \% \mathrm{MeOH}$ in $\mathrm{CH}_{2} \mathrm{Cl}_{2}$ in a $1 \mathrm{dram}$ vial, and pentane was used as the anti-solvent in a $20 \mathrm{~mL}$ scintillation vial. The crystallization chamber was placed in a refrigerator at $4{ }^{\circ} \mathrm{C}$. Yellow needles form within several days. The crystal structure of $\mathbf{A}$ was obtained from a crystal formed using this procedure (see X-ray section below).

Crude reaction mixture using $\mathbf{P 1}$ :

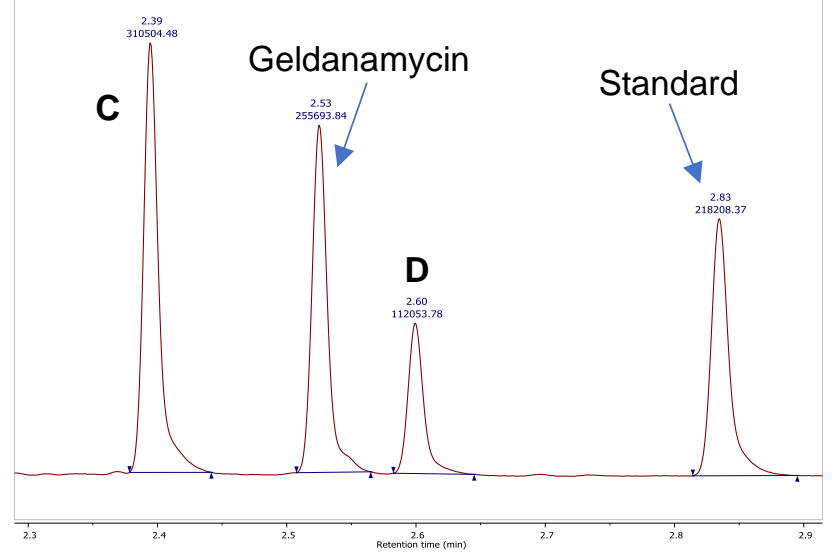

Purified C:

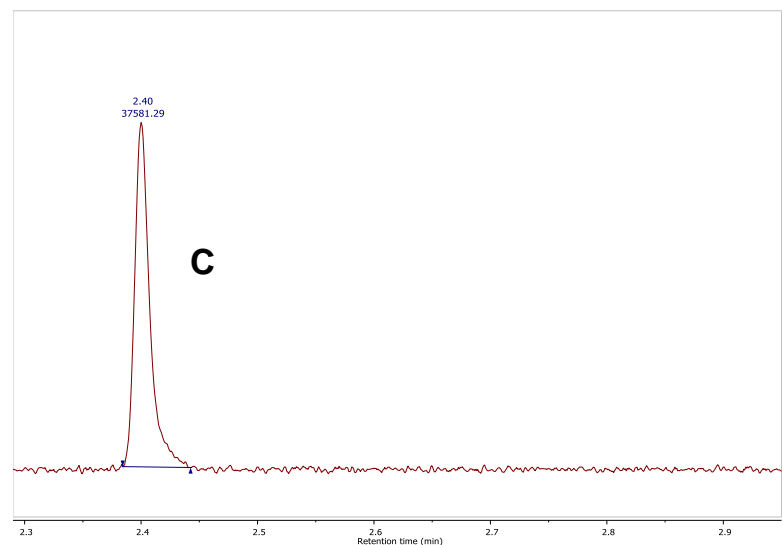

Purified D:

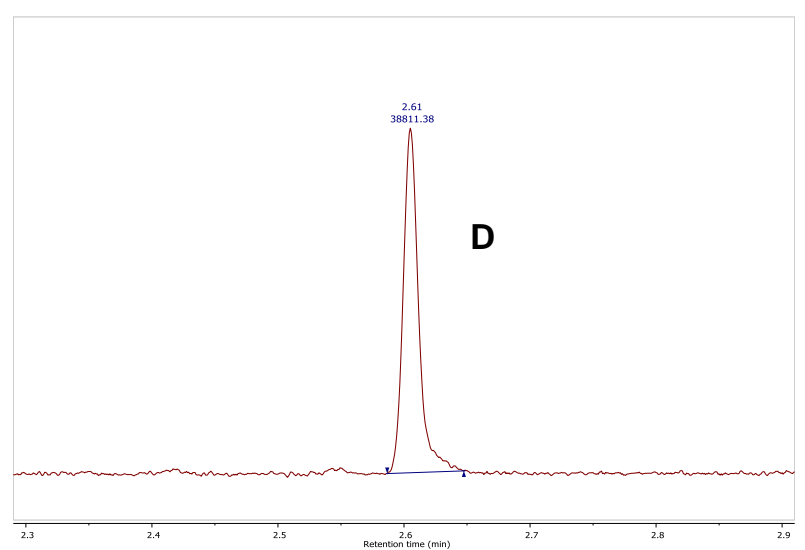


Crude reaction mixture using 17-AAG \& P10:

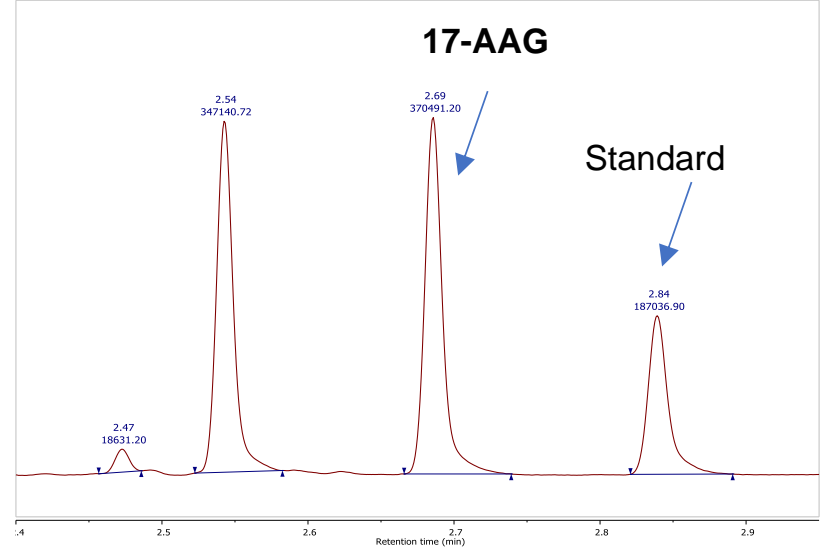

Purified major (17-A):

Purified minor (17-B):
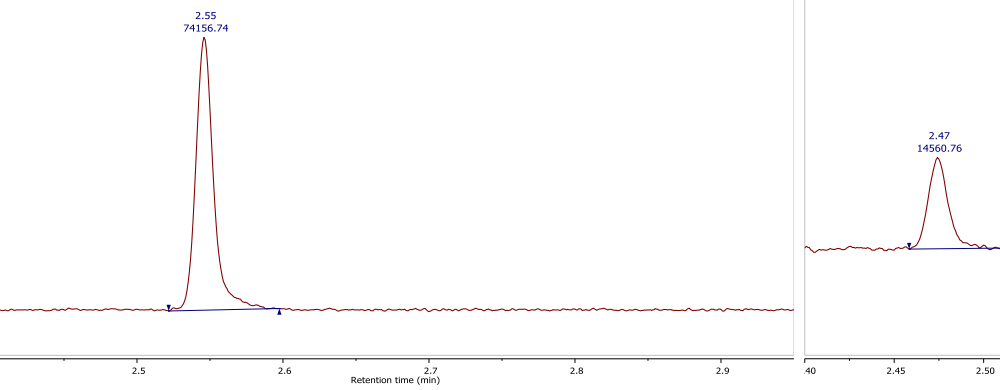

${ }_{2.9}^{1.9}$

Crude reaction mixture using $\mathbf{1 7 - A A G}$ and $\boldsymbol{m}$-CPBA ( $30 \%$ conversion):

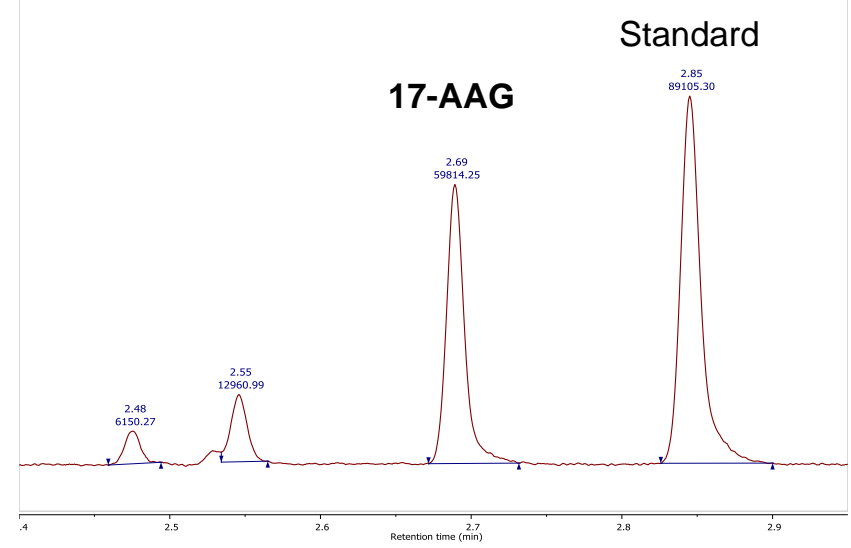


Crude reaction mixture using 17-AAG and P1 (10\% conversion):

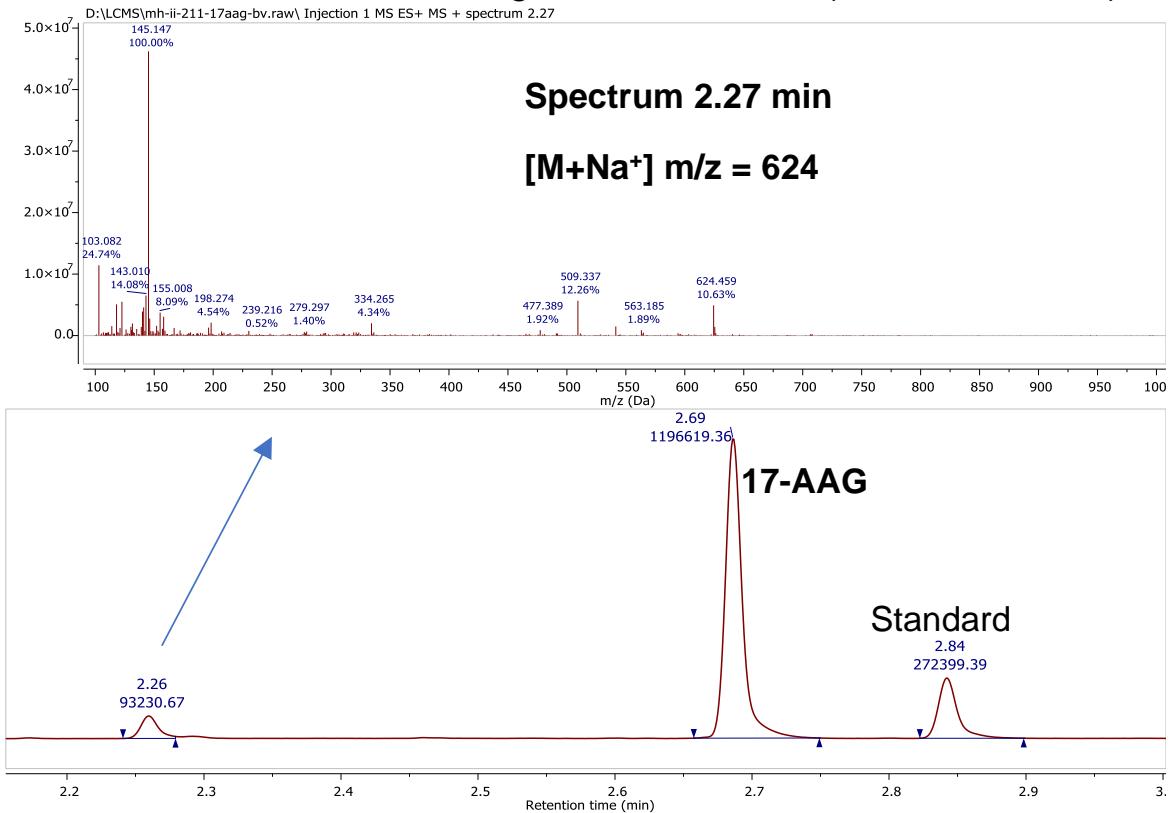




\section{Preparatory HPLC Purification Conditions}

Method: Gradient using MeCN (0.1\% formic acid) in $\mathrm{H}_{2} \mathrm{O}(0.1 \%$ formic acid) over 35 min at 15 $\mathrm{mL} / \mathrm{min}$ (see table below for gradients).

\begin{tabular}{|c|c|}
\hline Time (min) & $\%$ MeCN in $\mathrm{H}_{2} \mathrm{O}$ \\
\hline $0-4$ & $0-10$ \\
\hline $4-10$ & $10-30$ \\
\hline $10-25$ & $30-55$ \\
\hline $25-35$ & $55-95$ \\
\hline
\end{tabular}

Column: SymmetryPrep C8 (7 $\mu \mathrm{m}$ particle size, 19 × $300 \mathrm{~mm})$

\section{Example Chromatograms from Prep HPLC}
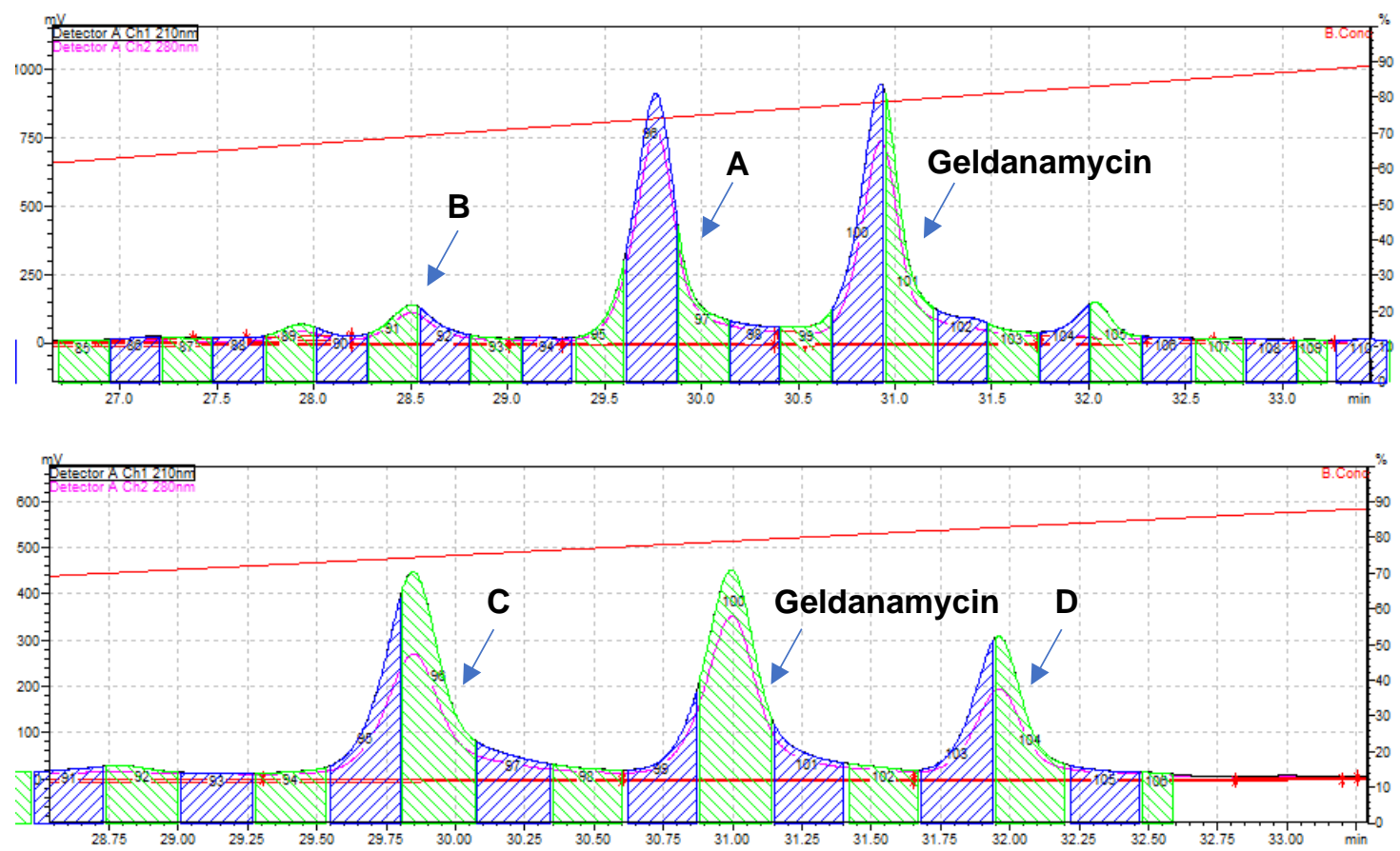
Structural Assignment of Product B - Comparison to Geldanamycin and Product A
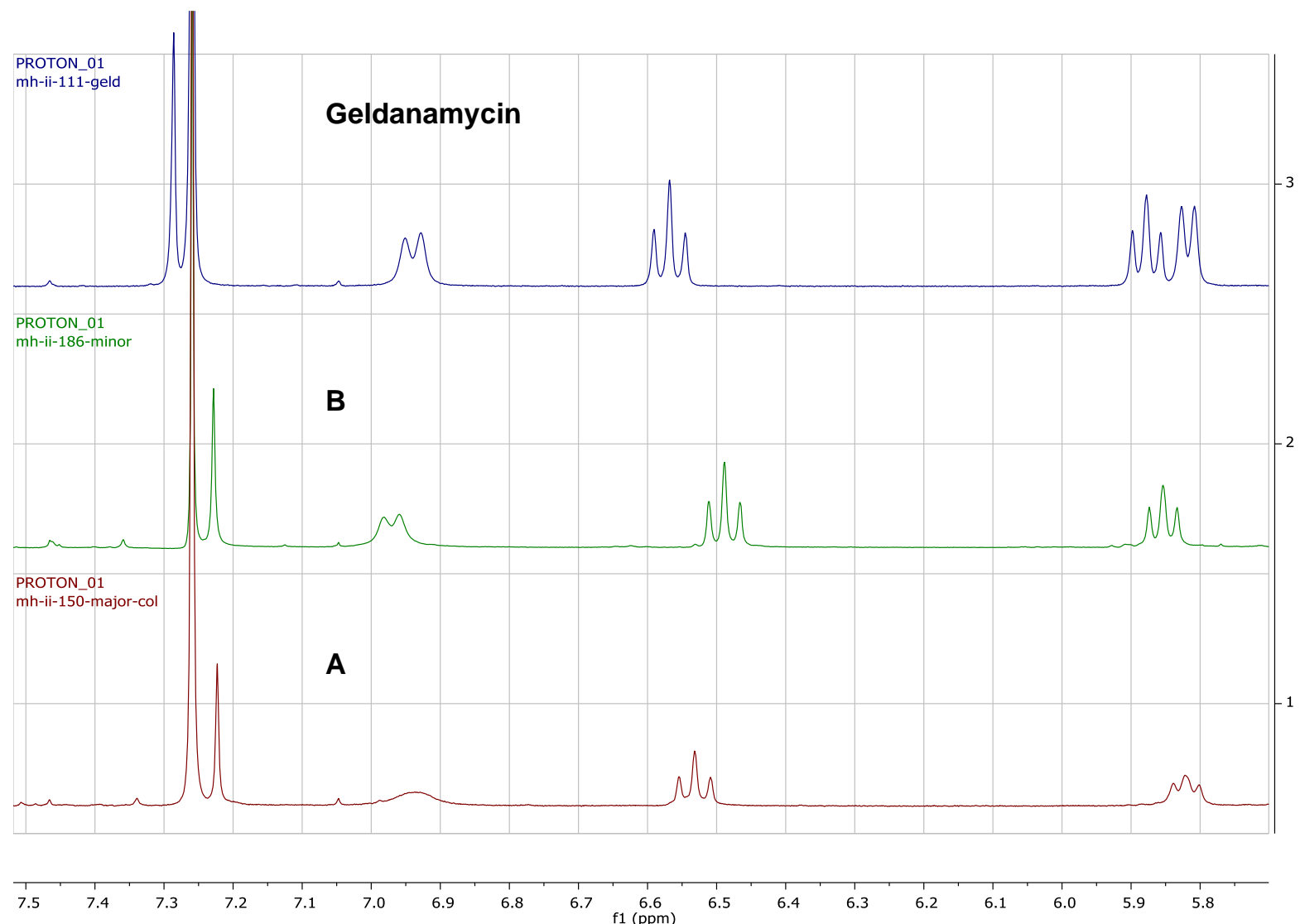

Product B has three alkenyl protons, which correlate to one another in a similar pattern as in $\mathbf{A}$ (see COSY spectra below). Thus, B was concluded to be an 8,9-epoxide yet opposite diastereomer to $\mathbf{A}$.
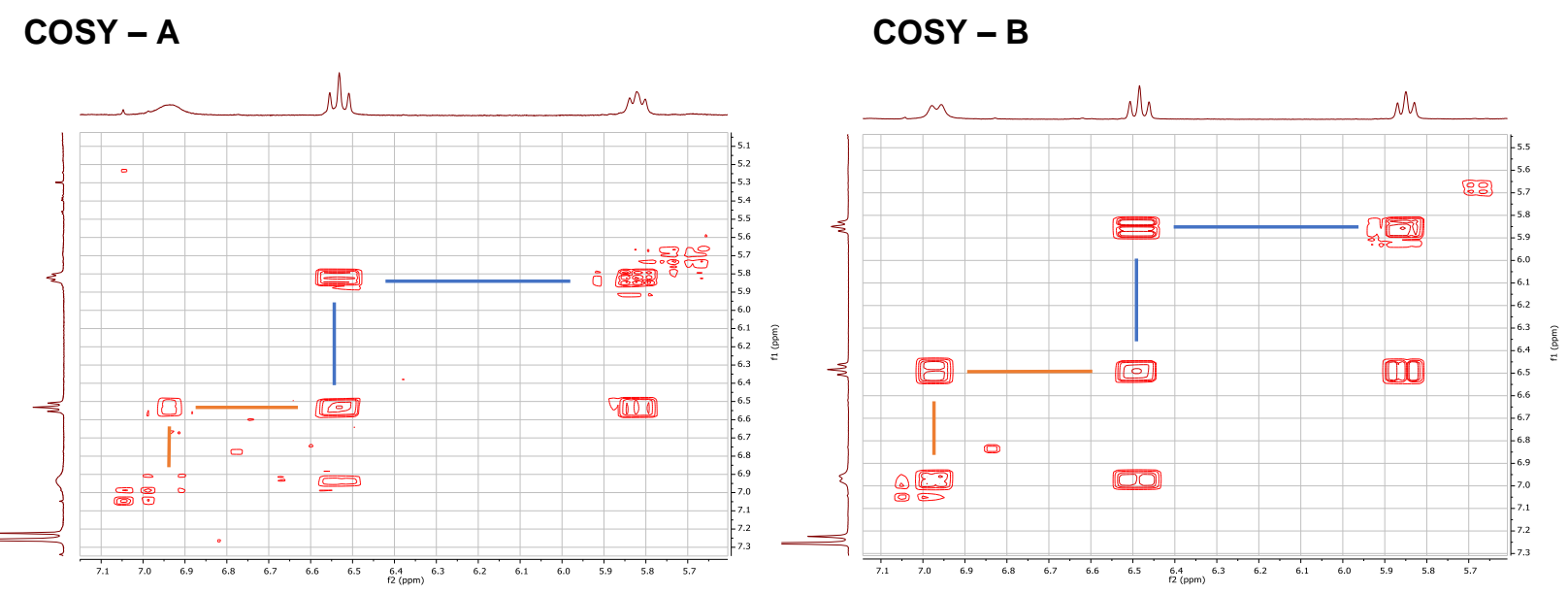


\section{Model Quinone Synthesis and Reactions}<smiles>COC1=CC(=O)C=CC1=O</smiles>

2-methoxy-1,4-benzoquinone was synthesized according to a reported procedure, and the spectra matched that of the previously reported. ${ }^{4}$<smiles>O=C1C=CC(=O)C(NC(=O)c2ccccc2)C1</smiles>

2-( $N$-benzamide)-1,4-benzoquinone was synthesized according to a reported procedure, and the spectra matched that of the previously reported. ${ }^{5}$<smiles>COC1CC(=O)C(NC(=O)c2ccccc2)CC1=O</smiles>

2-( $N$-benzamide)-5-methoxy-1,4-benzoquinone was synthesized according to a reported procedure. ${ }^{5}$

Using General Procedure C with P1 resulted in decomposition of each of the quinones. Using General Procedure D ( $m$-CPBA) resulted in no reaction of the quinones. General Procedure $\mathbf{E}$ (see below, with the respective quinone in place of geldanamycin) resulted in decomposition of each of the quinones.

\section{General Procedure E: Nucleophilic Epoxidation Reactions}

A standard solution of geldanamycin (2.8 $\mathrm{mg}$ or $5 \mu \mathrm{mol}$ per reaction, 1 equiv), including benzyl benzoate $\left(1.5 \mu \mathrm{L}\right.$ per reaction) as an internal standard, in $\mathrm{CH}_{2} \mathrm{Cl}_{2}$ was prepared so that the final

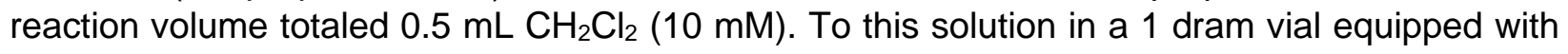
stir bar was added $t$-BuOOH ( $5 \mathrm{M}$ in decane, $1 \mu \mathrm{L}, 1$ equiv), followed by $\mathrm{DBU}$ ( 0.5 equiv, delivered from a standard solution in $\mathrm{CH}_{2} \mathrm{Cl}_{2}$ ). The reactions were stirred at ambient temperature for $24 \mathrm{~h}$, after which they were filtered through a short plug of $\mathrm{SiO}_{2}$ and $\mathrm{Na}_{2} \mathrm{SO}_{4}$ with $10 \% \mathrm{MeOH} / \mathrm{CH}_{2} \mathrm{Cl}_{2}$ and concentrated under reduced pressure. The reactions were prepared for analysis by UPLC/MS.

Using the above procedure, $77 \%$ conversion of geldanamycin afforded $61 \%$ C and $3 \%$ D. Compounds $\mathbf{A}$ and $\mathbf{B}$ were not observed. An additional product that is likely from hydrolysis of the 17-OMe group (m/z matches loss of OMe, plus $\mathrm{OH}$ ) was also observed in $12 \%$. 


\section{Biological Testing}

\section{Fluorescence Polarization}

Assay was performed in 96-well format in black, flat-bottom plates (Santa Cruz Biotechnology) with a final volume of $100 \mu \mathrm{L}$. Twenty-five microliters of assay buffer $(20 \mathrm{mM}$ HEPES, pH 7.3, $50 \mathrm{mM} \mathrm{KCl}, 5 \mathrm{mM} \mathrm{MgCl}, 20 \mathrm{mM} \mathrm{Na}_{2} \mathrm{MoO}_{4}, 2 \mathrm{mM}$ DTT, $0.1 \mathrm{mg} / \mathrm{mL} \mathrm{BGG}$, and $0.01 \% \mathrm{NP}-40$ ) containing $6 \mathrm{nM}$ FITC-GDA (fluorescent tracer, stock in DMSO and diluted in assay buffer) and $50 \mu \mathrm{L}$ of assay buffer containing $10 \mathrm{nM}$ of either Grp94, Hsp90a, or Hsp90ß were added to each well. Compounds were tested in triplicate wells (1\% DMSO final concentration). For each plate, wells containing buffer only (background), tracer in buffer only (low polarization control), and protein and tracer in buffer with $1 \%$ DMSO (high polarization control) were included. Plates were incubated at $4{ }^{\circ} \mathrm{C}$ with rocking for $24 \mathrm{~h}$. Polarization values (in $\mathrm{mP}$ units) were measured at $37^{\circ} \mathrm{C}$ with an excitation filter at $485 \mathrm{~nm}$ and an emission filter at $528 \mathrm{~nm}$. Polarization values were correlated to \% tracer bound and compound concentrations. The concentration at which the tracer was $50 \%$ displaced by the inhibitor was determined using Graphpad Prism.

\section{Anti-proliferative assay for MCF-7 and SkBr3 cells}

Cells were grown to confluence and seeded at 2000 cells/well $/ 0.1 \mathrm{~mL}$ in a 96 -well plate and placed back in the incubator for $24 \mathrm{~h}$. Compounds or vehicle were administered at the desired concentrations (1\% DMSO final concentration) and incubated for $72 \mathrm{~h}$. The percentage of viable cells was determined using the MTS/PMS cell proliferation kit (Promega) per the manufacturer's instructions. Cells treated with vehicle were normalized to $100 \%$ proliferation and values adjusted accordingly.

\section{Western blot for SkBr-3 cells}

SkBr-3 cells were seeded into $10 \mathrm{~cm}$ dishes and grown to $70 \%$ confluence. Compound (administered as a solution in DMSO) or vehicle was diluted into $5 \mathrm{~mL}$ fresh media (assay concentration of $1 \%$ DMSO) and added to the dishes, which were returned to the incubator for 24 h. Cells were harvested in cold PBS and lysed using MPER (Thermo Scientific) supplemented with protease and phosphatase inhibitors (Roche) per manufacturer's directions. Lysates were clarified at $14000 \mathrm{~g}$ for $15 \mathrm{~m}$ at $4{ }^{\circ} \mathrm{C}$. Protein concentrations were determined using the Pierce $\mathrm{BCA}$ protein assay kit per the manufacturer's instructions. Equal amounts of protein $(10 \mu \mathrm{g})$ were electrophoresed under reducing conditions (10\% tris-glycine gels), transferred to PVDF (Millipore ImmobilonP), and immunoblotted with the corresponding primary antibody. Membranes were incubated with an appropriate horseradish peroxidase-labeled secondary antibody, developed with a chemiluminescent substrate (Bio-Rad Clarity), and visualized with autoradiography film (Midsci). 
VIII. NMR Spectra
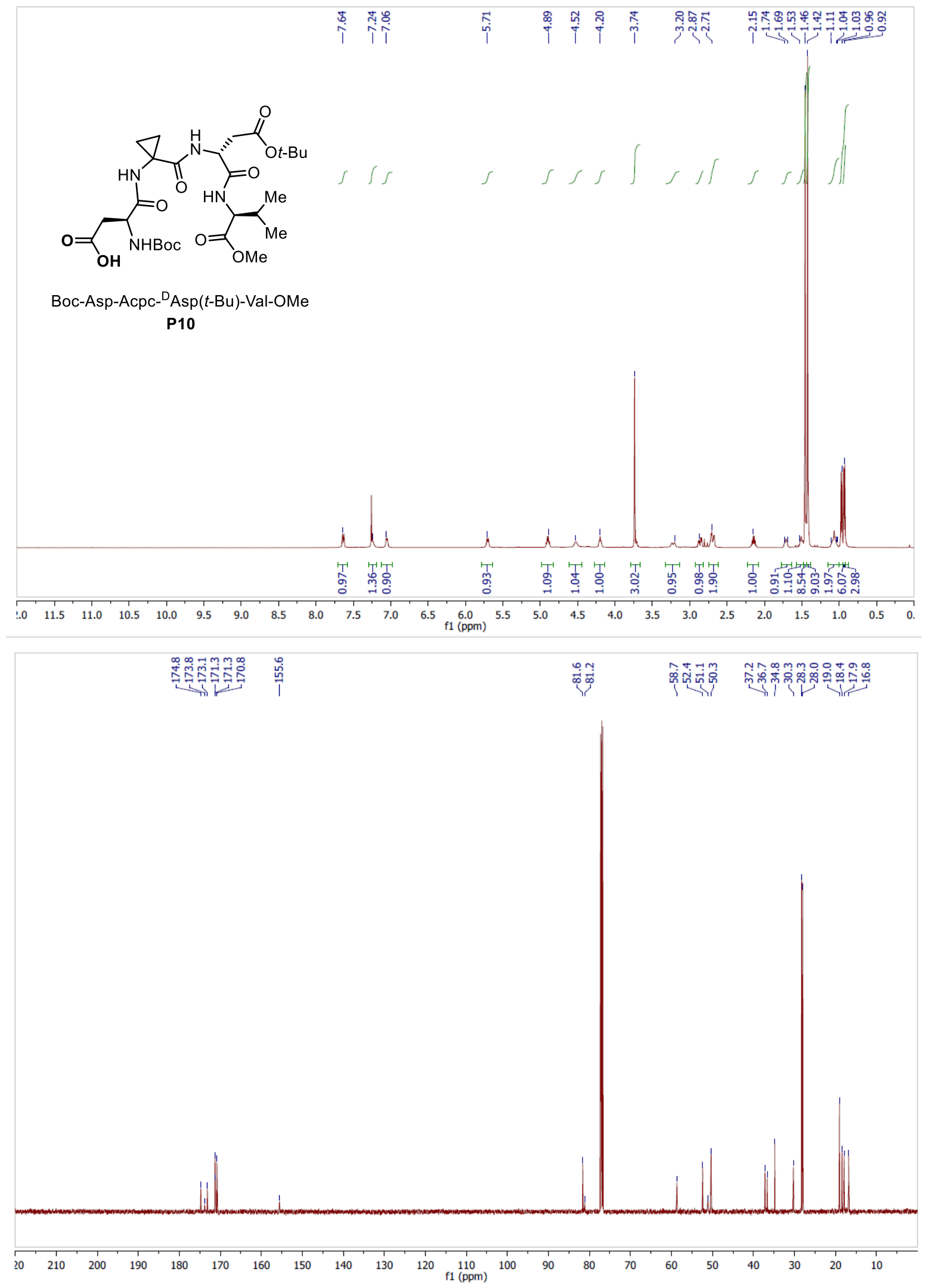

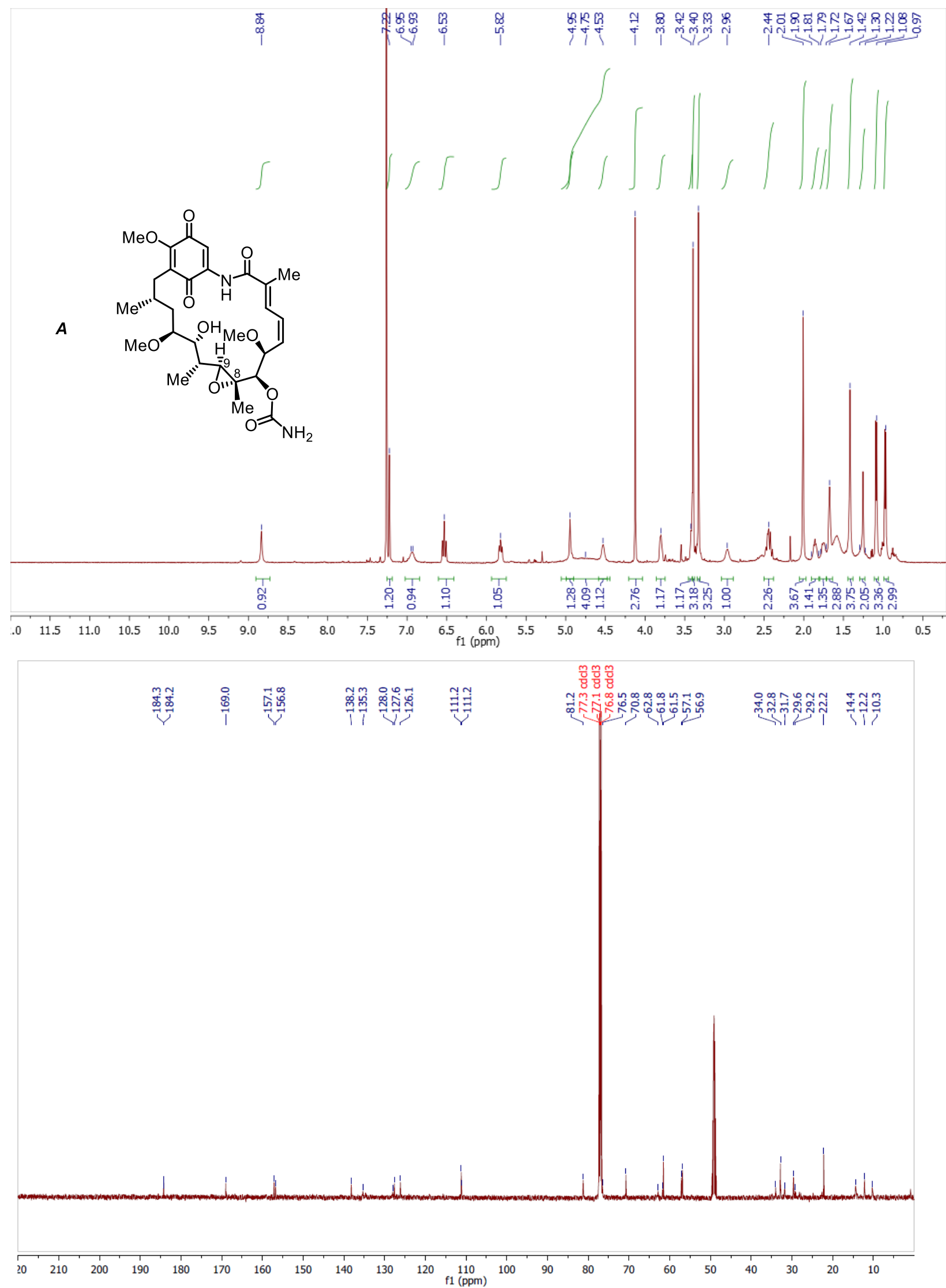


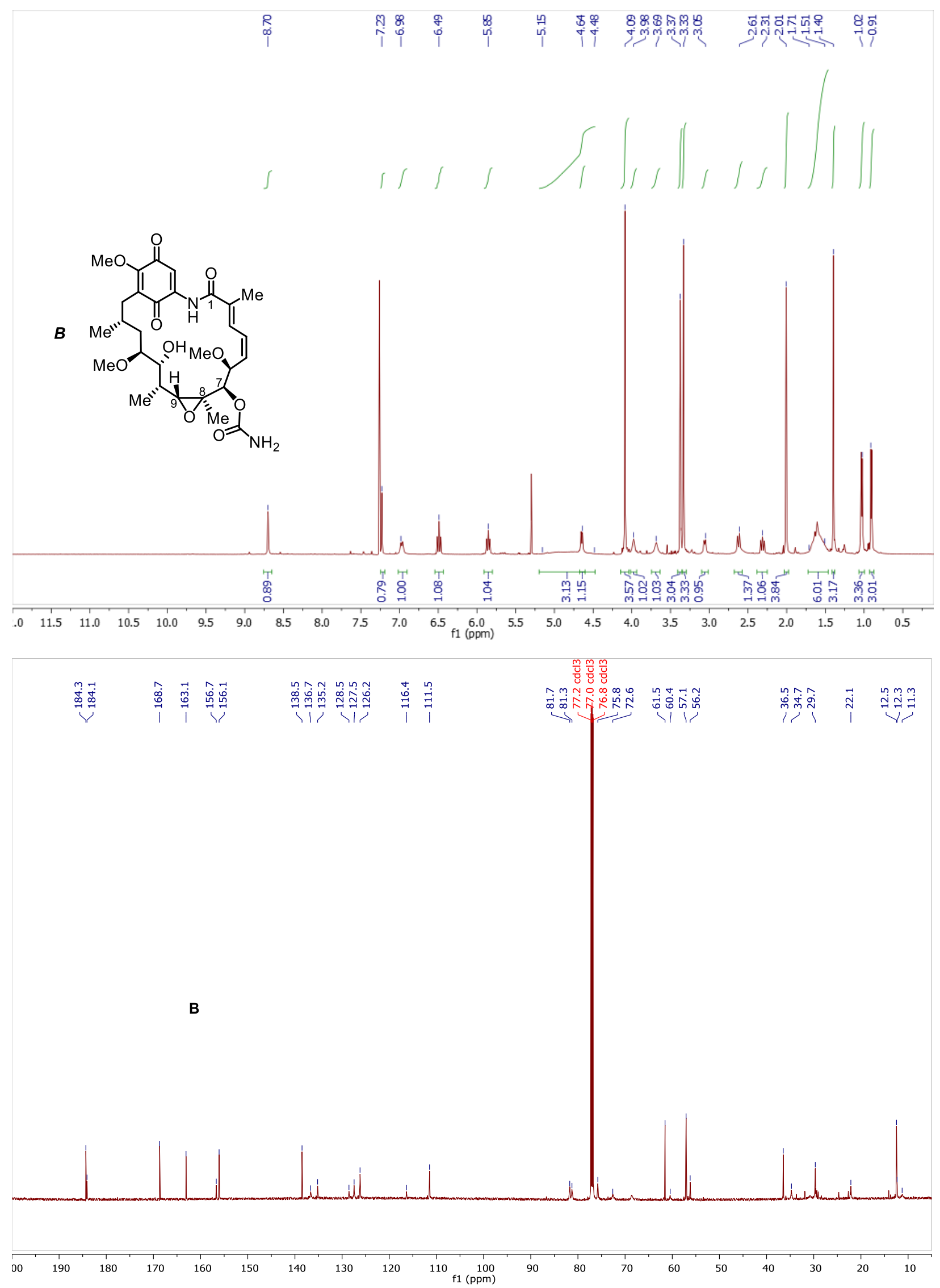



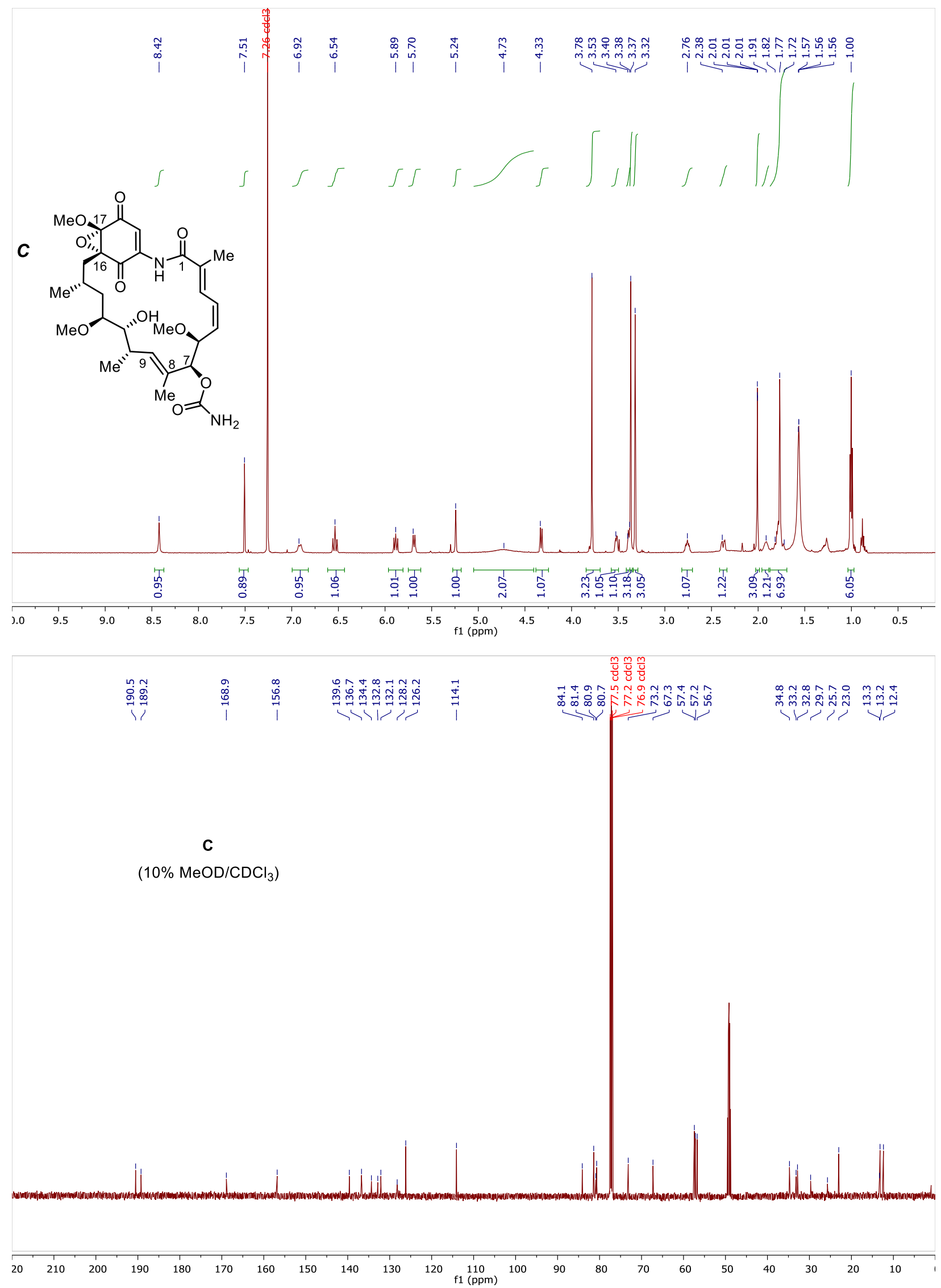

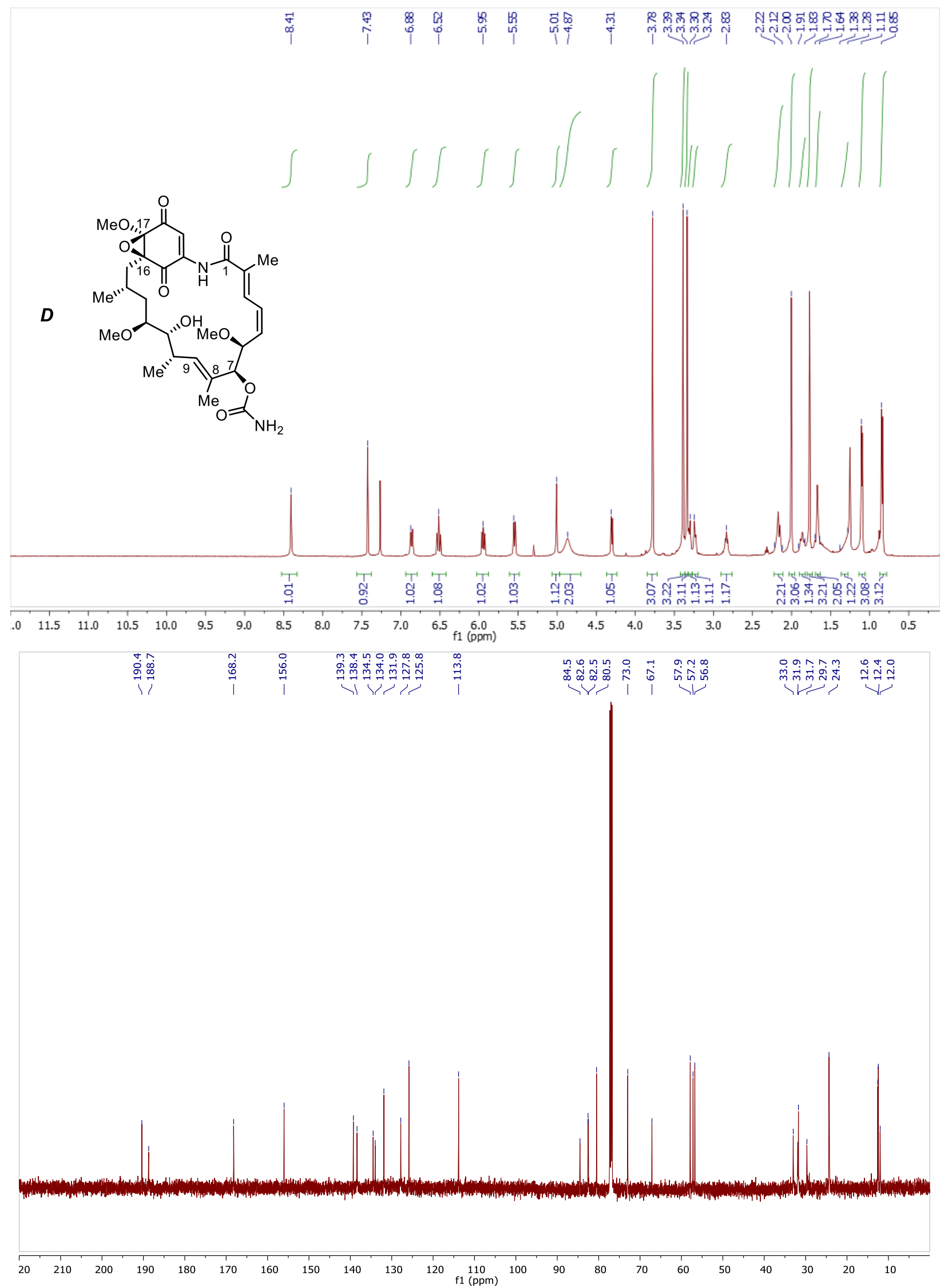


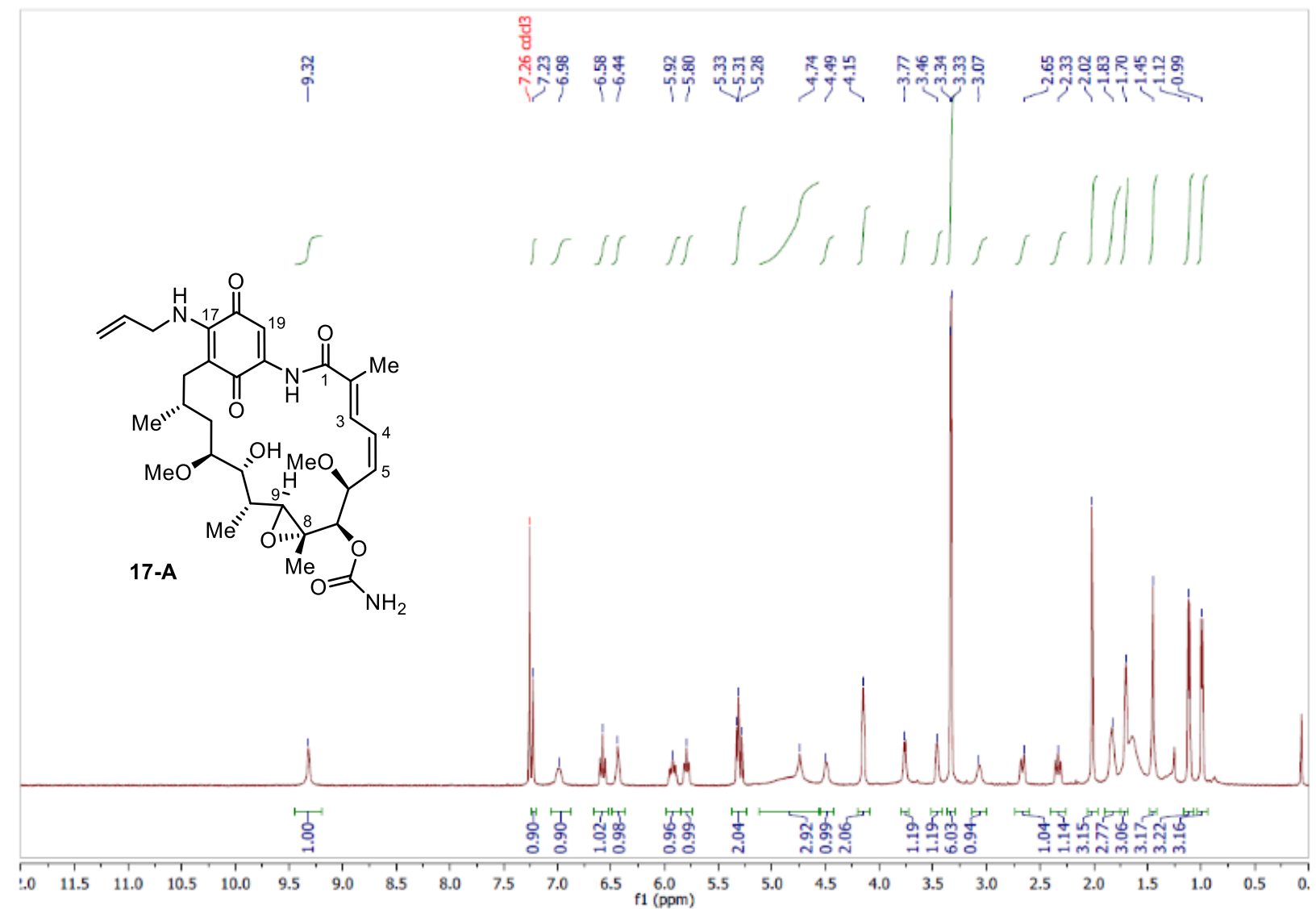

17-A

$\left(10 \% \mathrm{MeOD}-\mathrm{d}_{4} / \mathrm{CDCl}_{3}\right)$
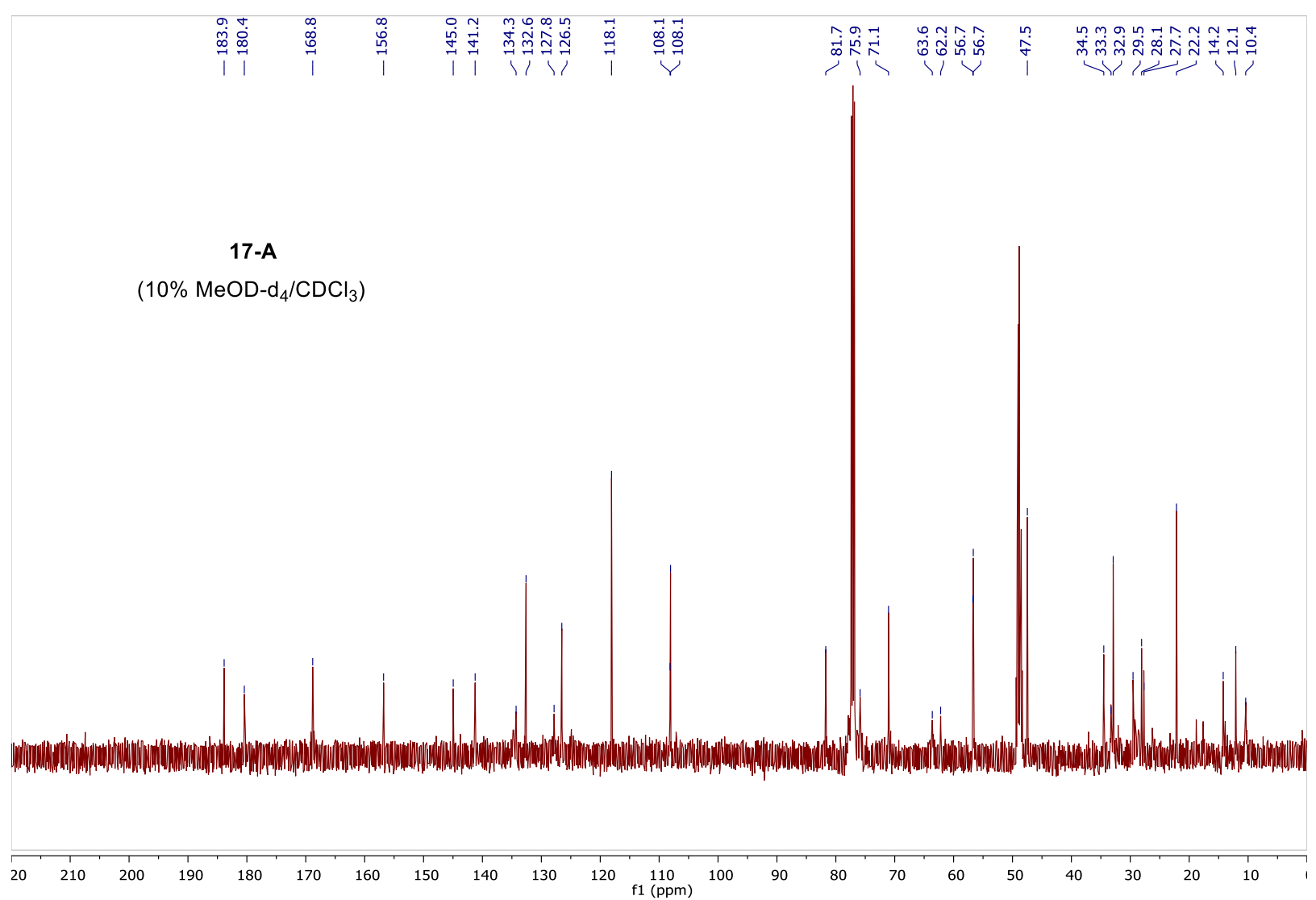


\section{X-Ray Crystallography Data}

Low-temperature diffraction data ( $\omega$-scans) were collected on a Rigaku MicroMax-007HF diffractometer coupled to a Saturn994+ CCD detector with $\mathrm{Cu} \mathrm{Ka}(\lambda=1.54178 \AA)$ for the structures A, C, D, and 17-A. All diffraction images were processed and scaled using Rigaku Oxford Diffraction software. ${ }^{6}$ The structure was solved with SHELXT and was refined against $F^{2}$ on all data by full-matrix least squares with SHELXL. ${ }^{7}$ All non-hydrogen atoms were refined anisotropically. Hydrogen atoms were included in the model at geometrically calculated positions and refined using a riding model. Unless stated otherwise, the isotropic displacement parameters of all hydrogen atoms were fixed to 1.2 times the $U$ value of the atoms to which they are linked (1.5 times for methyl groups).

The full numbering scheme of all compounds can be found in the full details of the X-ray structure determination (CIF), which is included as Supporting Information. CCDC numbers: 1964613 (Compound A), 1964602 (Compound C), 1964609 (Compound D), and 1964610 (Compound 17A). These data can be obtained free of charge from The Cambridge Crystallographic Data Center via www.ccdc.cam.ac.uk/data_request/cif. 
Table S3. Details of Crystal Structures

\begin{tabular}{|c|c|c|c|c|}
\hline Compound & $\mathbf{A}$ & $\mathbf{C}$ & D & 17-A \\
\hline Data Code & 007b-19042 & $007 a-19010$ & 007b-19137 & 007b-19118 \\
\hline CCDC Number & 1964613 & 1964602 & 1964609 & 1960610 \\
\hline $\begin{array}{c}\text { Empirical } \\
\text { Formula }\end{array}$ & $\mathrm{C}_{29} \mathrm{H}_{40} \mathrm{~N}_{2} \mathrm{O}_{10}$ & $\mathrm{C}_{29} \mathrm{H}_{40} \mathrm{~N}_{2} \mathrm{O}_{10}$ & $\mathrm{C}_{29} \mathrm{H}_{40} \mathrm{~N}_{2} \mathrm{O}_{10}$ & $\mathrm{C}_{31} \mathrm{H}_{43} \mathrm{~N}_{3} \mathrm{O}_{9}$ \\
\hline Temperature (K) & $93(2)$ & $93(2)$ & $93(2)$ & $93(2)$ \\
\hline Wavelength $(\AA)$ & 1.54184 & 1.54184 & 1.54184 & 1.54184 \\
\hline Crystal System & Monoclinic & Monoclinic & Orthorhombic & Monoclinic \\
\hline Space Group & $P 2_{1}$ & $P 2_{1}$ & $P 2{ }_{1} 2_{1}{ }_{1}$ & $P 2_{1}$ \\
\hline 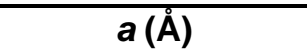 & 16.1463(3) & $12.5726(4)$ & $8.1886(2)$ & $15.2434(7)$ \\
\hline$b(\AA)$ & $7.84730(10)$ & $7.5849(2)$ & $18.7570(3)$ & $7.8592(2)$ \\
\hline$c(\AA)$ & $30.1019(5)$ & $16.0036(4)$ & $20.4466(4)$ & $15.5165(9)$ \\
\hline$\alpha\left({ }^{\circ}\right)$ & 90 & 90 & 90 & 90 \\
\hline$\beta\left({ }^{\circ}\right)$ & 103.866(2) & 99.298(3) & 90 & $105.045(5)$ \\
\hline$Y\left({ }^{\circ}\right)$ & 90 & 90 & 90 & 90 \\
\hline$V\left(\AA^{3}\right)$ & 3702.91(11) & $1506.08(7)$ & 3140.47 & $1795.17(15)$ \\
\hline$Z$ & 4 & 2 & 4 & 2 \\
\hline Density $\left(\mathrm{g} / \mathrm{cm}^{3}\right)$ & 1.034 & 1.272 & 1.220 & 1.113 \\
\hline $\begin{array}{c}\text { Absorption } \\
\text { coefficient }\left(\mathrm{mm}^{-1}\right)\end{array}$ & 0.650 & 0.799 & 0.767 & 0.676 \\
\hline Completeness & $99.9 \%$ & $99.9 \%$ & $99.5 \%$ & $99.5 \%$ \\
\hline $\begin{array}{c}\text { Data / restraints / } \\
\text { parameters }\end{array}$ & 12841 / 83 / 833 & 5277 / 1 / 378 & 5520 / 0 / 393 & 5277 / 1 / 378 \\
\hline$R 1, \mathrm{w} R 2(\mathrm{I}>2 \sigma(\mathrm{I}))$ & $0.0372,0.1054$ & $0.0596,0.1511$ & $0.0365,0.0941$ & $0.0910,0.2251$ \\
\hline$R 1, w R 2$ (all data) & $0.0383,0.1064$ & $0.0651,0.1558$ & $0.0410,0.0968$ & $0.1318,0.2609$ \\
\hline GOF & 1.056 & 1.064 & 1.037 & 1.048 \\
\hline $\begin{array}{l}\text { Absolute } \\
\text { structure } \\
\text { parameter }\end{array}$ & $1.25(6)$ & $0.05(10)$ & $-0.03(6)$ & $-0.5(3)$ \\
\hline $\begin{array}{c}\text { Largest Diff. } \\
\text { Peak, Hole }\left(\mathrm{e} / \AA^{3}\right)\end{array}$ & $0.228,-0.159$ & $0.321,-0.260$ & $0.168,-0.196$ & $0.886,-0.314$ \\
\hline
\end{tabular}




\section{Refinement details for $\mathbf{A}$ :}

The quinone and methoxy groups in both models are disordered over two positions. The site occupancies were freely refined for both models. All chemically similar bonds were restrained to have similar distances. The quinone groups were restrained to behave as ridged bodies. $\mathrm{C} 14$ and C14B are very close in proximity and required the minor occupancy atom, C14B, be constrained to the same atomic displacement parameter as the major, C14. The program SQUEEZE ${ }^{8}$ was used to compensate for the contribution of disordered solvents contained in voids within the crystal lattice from the diffraction intensities. This procedure was applied to the data file and the submitted model is based on the solvent removed data. Based on the total electron density found in the voids $\left(305 \mathrm{e} / \AA^{3}\right)$, it is likely that $\sim 2$ dichloromethane molecules are present in the unit cell. See "_platon_squeeze_details" in the .cif for more information.

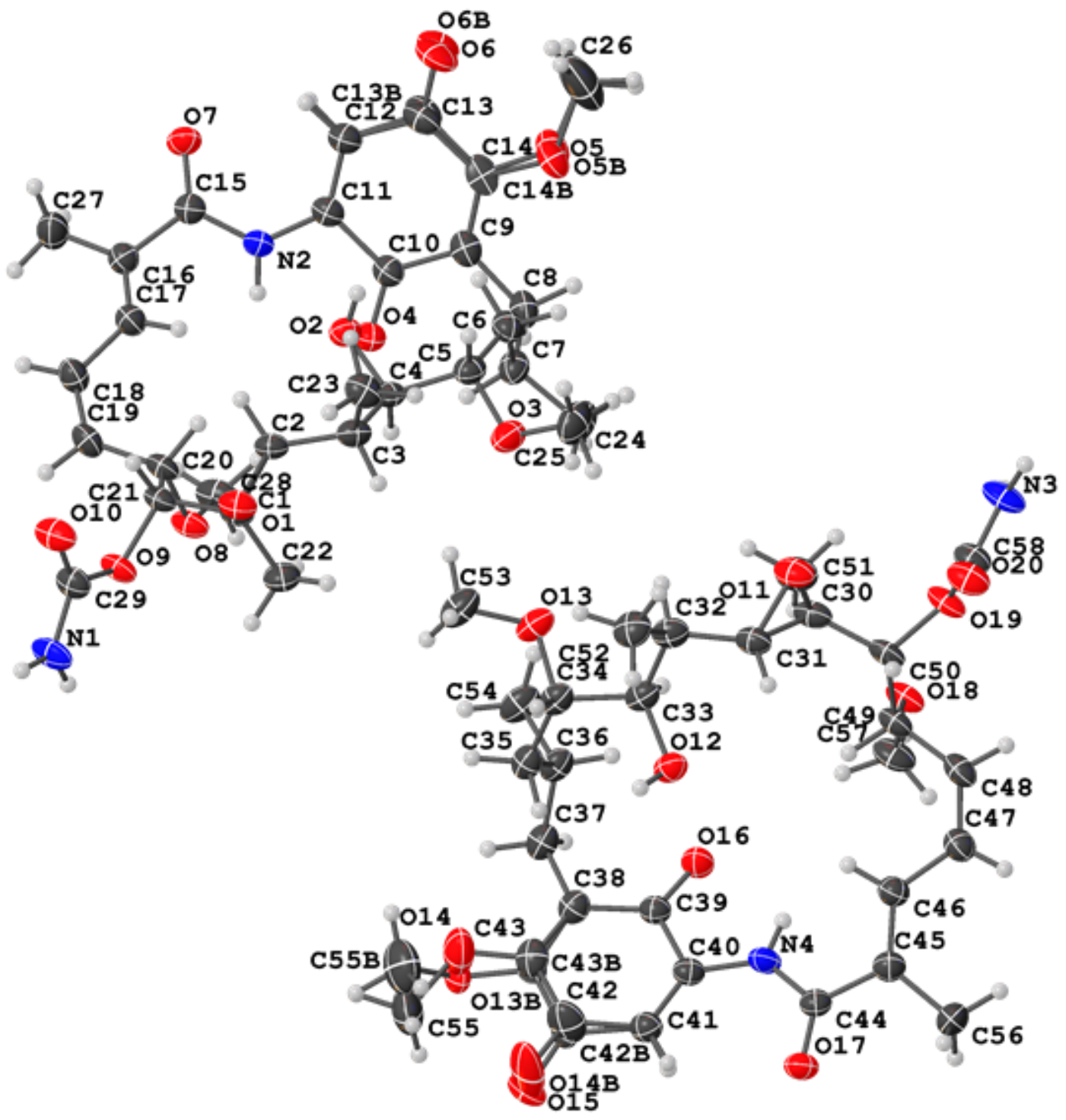

Figure S1. The complete numbering scheme of $\mathbf{A}$ with $50 \%$ thermal ellipsoid probability levels. The hydrogen atoms are shown as circles for clarity. 
Table S4. Atomic coordinates $\left(x 10^{4}\right)$ and equivalent isotropic displacement parameters $\left(\AA^{2} x\right.$ $10^{3}$ ) for $\mathbf{A}$. U(eq) is defined as one third of the trace of the orthogonalized Uij tensor.

\begin{tabular}{|c|c|c|c|c|}
\hline & $x$ & $y$ & $z$ & $\mathrm{U}(\mathrm{eq})$ \\
\hline $\mathrm{O}(1)$ & $10060(1)$ & $4525(2)$ & 7219(1) & $36(1)$ \\
\hline $\mathrm{O}(2)$ & 10906(1) & 2328(2) & $6103(1)$ & $33(1)$ \\
\hline $\mathrm{O}(3)$ & $12915(1)$ & $2450(3)$ & $6963(1)$ & $42(1)$ \\
\hline $\mathrm{O}(4)$ & $10765(1)$ & $-1894(3)$ & $5830(1)$ & $37(1)$ \\
\hline $\mathrm{O}(5)$ & $12857(8)$ & $-390(17)$ & $5070(5)$ & $43(2)$ \\
\hline $\mathrm{O}(5 \mathrm{~B})$ & $12791(8)$ & $-880(20)$ & $4967(5)$ & $50(2)$ \\
\hline $\mathrm{O}(6)$ & $11508(4)$ & $662(17)$ & $4360(3)$ & $54(2)$ \\
\hline $\mathrm{O}(6 \mathrm{~B})$ & $11425(5)$ & $-210(20)$ & $4235(3)$ & $59(3)$ \\
\hline $\mathrm{O}(7)$ & $8562(1)$ & $-415(3)$ & $4499(1)$ & $37(1)$ \\
\hline $\mathrm{O}(8)$ & $9007(1)$ & $-628(2)$ & $6876(1)$ & $34(1)$ \\
\hline $\mathrm{O}(9)$ & $8507(1)$ & 2138(2) & 7281(1) & $34(1)$ \\
\hline $\mathrm{O}(10)$ & $8017(1)$ & 4859(3) & $7207(1)$ & $44(1)$ \\
\hline$N(1)$ & $7931(2)$ & $3207(3)$ & $7815(1)$ & $43(1)$ \\
\hline$N(2)$ & $9476(1)$ & $-948(3)$ & $5187(1)$ & $33(1)$ \\
\hline$C(1)$ & 9883(2) & $2729(3)$ & $7126(1)$ & $30(1)$ \\
\hline$C(2)$ & $10312(2)$ & $3739(3)$ & $6834(1)$ & $29(1)$ \\
\hline$C(3)$ & $11254(2)$ & 3744(3) & $6855(1)$ & $33(1)$ \\
\hline$C(4)$ & $11482(2)$ & 2353(3) & $6547(1)$ & $30(1)$ \\
\hline$C(5)$ & $12407(2)$ & $2521(3)$ & $6506(1)$ & $34(1)$ \\
\hline$C(6)$ & $12668(2)$ & $1230(4)$ & $6183(1)$ & $36(1)$ \\
\hline$C(7)$ & $12596(2)$ & $-660(4)$ & $6295(1)$ & $36(1)$ \\
\hline$C(8)$ & $12537(2)$ & $-1775(4)$ & $5860(1)$ & $38(1)$ \\
\hline $\mathrm{C}(9)$ & $11854(2)$ & $-1268(3)$ & $5449(1)$ & $35(1)$ \\
\hline$C(10)$ & $10968(2)$ & $-1379(3)$ & $5484(1)$ & $32(1)$ \\
\hline$C(11)$ & $10259(2)$ & $-851(3)$ & $5080(1)$ & $31(1)$ \\
\hline$C(12)$ & $10431(2)$ & $-363(4)$ & $4684(1)$ & $36(1)$ \\
\hline$C(13)$ & $11328(7)$ & $-70(20)$ & $4680(4)$ & $39(3)$ \\
\hline$C(13 B)$ & $11297(7)$ & $-480(20)$ & $4613(4)$ & $39(3)$ \\
\hline$C(14)$ & $12037(9)$ & $-530(20)$ & $5077(4)$ & $29(2)$ \\
\hline$C(14 B)$ & 11993(9) & $-950(20)$ & $5023(4)$ & $29(2)$ \\
\hline$C(15)$ & $8685(2)$ & $-580(3)$ & $4914(1)$ & $31(1)$ \\
\hline
\end{tabular}




$\begin{array}{lrrrr}\mathrm{C}(16) & 7988(2) & -372(3) & 5158(1) & 32(1) \\ \mathrm{C}(17) & 8188(2) & 148(4) & 5593(1) & 34(1) \\ \mathrm{C}(18) & 7610(2) & 421(4) & 5889(1) & 38(1) \\ \mathrm{C}(19) & 7873(2) & 758(4) & 6338(1) & 36(1) \\ \mathrm{C}(20) & 8780(2) & 867(3) & 6605(1) & 30(1) \\ \mathrm{C}(21) & 8945(2) & 2412(3) & 6921(1) & 29(1) \\ \mathrm{C}(22) & 10347(2) & 1537(4) & 7489(1) & 40(1) \\ \mathrm{C}(23) & 11498(2) & 5550(4) & 6744(1) & 39(1) \\ \mathrm{C}(24) & 13741(2) & 3148(5) & 7018(1) & 54(1) \\ \mathrm{C}(25) & 13355(2) & -1278(4) & 6669(1) & 49(1) \\ \mathrm{C}(26) & 13113(3) & -279(9) & 4632(2) & 99(2) \\ \mathrm{C}(27) & 7106(2) & -695(5) & 4873(1) & 46(1) \\ \mathrm{C}(28) & 9150(2) & -2090(4) & 6618(1) & 45(1) \\ \mathrm{C}(29) & 8138(2) & 3532(3) & 7424(1) & 35(1) \\ \mathrm{O}(11) & 16086(1) & -376(2) & 7942(1) & 41(1) \\ \mathrm{O}(12) & 14670(1) & -2229(3) & 8861(1) & 36(1) \\ \mathrm{O}(13) & 12977(1) & -1993(3) & 7822(1) & 42(1) \\ \mathrm{O}(13 \mathrm{~B}) & 12059(6) & -5798(12) & 9622(4) & 35(2) \\ \mathrm{O}(14) & 12118(4) & -5109(16) & 9518(3) & 63(2) \\ \mathrm{O}(14 \mathrm{~B}) & 13058(8) & -5053(11) & 10472(4) & 39(2) \\ \mathrm{O}(15) & 13108(5) & -4390(20) & 10400(3) & 92(4) \\ \mathrm{O}(16) & 14652(1) & -6504(3) & 9151(1) & 36(1) \\ \mathrm{O}(17) & 16218(1) & -4988(2) & 10667(1) & 35(1) \\ \mathrm{O}(18) & 16981(1) & -5526(2) & 8454(1) & 36(1) \\ \mathrm{O}(19) & 17631(1) & -2855(2) & 8119(1) & 37(1) \\ \mathrm{O}(20) & 18133(1) & -136(3) & 8213(1) & 44(1) \\ \mathrm{N}(3) & 18293(2) & -1809(3) & 7617(1) & 45(1) \\ \mathrm{N}(4) & 15624(1) & -5557(3) & 9918(1) & 34(1) \\ \mathrm{C}(30) & 16166(2) & -2160(3) & 8087(1) & 35(1) \\ \mathrm{C}(31) & 15626(2) & -964(3) & 8272(1) & 34(1) \\ \mathrm{C}(32) & 14666(2) & -855(4) & 8128(1) & 37(1) \\ \mathrm{C}(33) & 14270(2) & -2204(3) & 8381(1) & 33(1) \\ \mathrm{C}(34) & -3307(3) & 8564(1) & 35(1) \\ \mathrm{C}(35) & -5185(3) & 8470(1) & 35(1) \\ \mathrm{C}(36) & -6351(4) & 8842(1) & 38(1) \\ \mathrm{C}(37) & & & & \end{array}$




\begin{tabular}{|c|c|c|c|c|}
\hline$C(38)$ & $13327(2)$ & $-5917(3)$ & $9322(1)$ & $35(1)$ \\
\hline$C(39)$ & $14257(2)$ & $-6018(3)$ & $9431(1)$ & $30(1)$ \\
\hline$C(40)$ & $14763(2)$ & $-5503(3)$ & $9897(1)$ & $29(1)$ \\
\hline$C(41)$ & $14373(2)$ & $-5051(3)$ & 10226(1) & $34(1)$ \\
\hline$C(42)$ & $13464(7)$ & $-4890(20)$ & $10107(4)$ & $54(5)$ \\
\hline $\mathrm{C}(42 \mathrm{~B})$ & $13422(8)$ & $-5210(30)$ & $10152(5)$ & $29(3)$ \\
\hline $\mathrm{C}(43)$ & $12959(7)$ & $-5269(19)$ & $9638(4)$ & $39(3)$ \\
\hline$C(43 B)$ & $12925(10)$ & $-5690(30)$ & $9678(5)$ & $26(3)$ \\
\hline $\mathrm{C}(44)$ & $16301(2)$ & $-5153(3)$ & $10276(1)$ & $30(1)$ \\
\hline$C(45)$ & $17138(2)$ & $-4929(3)$ & $10158(1)$ & $34(1)$ \\
\hline$C(46)$ & $17153(2)$ & $-4508(3)$ & $9727(1)$ & $34(1)$ \\
\hline$C(47)$ & $17894(2)$ & $-4250(4)$ & $9541(1)$ & $37(1)$ \\
\hline$C(48)$ & $17847(2)$ & $-4023(4)$ & $9094(1)$ & $37(1)$ \\
\hline$C(49)$ & $17050(2)$ & $-4007(3)$ & $8717(1)$ & $31(1)$ \\
\hline$C(50)$ & $17027(2)$ & $-2498(3)$ & $8395(1)$ & $33(1)$ \\
\hline$C(51)$ & $15806(2)$ & $-3419(4)$ & $7715(1)$ & $47(1)$ \\
\hline$C(52)$ & $14405(2)$ & 979(4) & $8203(1)$ & $44(1)$ \\
\hline$C(53)$ & 12162(2) & $-1234(4)$ & $7682(1)$ & $49(1)$ \\
\hline$C(54)$ & $12501(2)$ & $-5751(4)$ & $8001(1)$ & $44(1)$ \\
\hline$C(55)$ & $11608(4)$ & $-4883(16)$ & $9844(4)$ & $75(3)$ \\
\hline$C(55 B)$ & $11561(8)$ & $-4262(18)$ & $9611(8)$ & $71(5)$ \\
\hline$C(56)$ & $17908(2)$ & $-5120(5)$ & $10549(1)$ & $50(1)$ \\
\hline$C(57)$ & $16768(2)$ & $-6995(4)$ & $8684(1)$ & $44(1)$ \\
\hline$C(58)$ & $18030(2)$ & $-1451(3)$ & $7992(1)$ & $36(1)$ \\
\hline
\end{tabular}

Table S5. Bond lengths $[\AA]$ and angles $\left[^{\circ}\right]$ for $\mathbf{A}$.

\begin{tabular}{ll}
\hline $\mathrm{O}(1)-\mathrm{C}(1)$ & $1.452(3)$ \\
$\mathrm{O}(1)-\mathrm{C}(2)$ & $1.454(3)$ \\
$\mathrm{O}(2)-\mathrm{C}(4)$ & $1.433(3)$ \\
$\mathrm{O}(2)-\mathrm{H}(2)$ & 0.8400 \\
$\mathrm{O}(3)-\mathrm{C}(24)$ & $1.413(4)$ \\
$\mathrm{O}(3)-\mathrm{C}(5)$ & $1.425(3)$ \\
$\mathrm{O}(4)-\mathrm{C}(10)$ & $1.232(3)$
\end{tabular}




$\begin{array}{ll}\mathrm{O}(5)-\mathrm{C}(14) & 1.335(12) \\ \mathrm{O}(5)-\mathrm{C}(26) & 1.475(15) \\ \mathrm{O}(5 \mathrm{~B})-\mathrm{C}(26) & 1.327(16) \\ \mathrm{O}(5 \mathrm{~B})-\mathrm{C}(14 \mathrm{~B}) & 1.340(12) \\ \mathrm{O}(6)-\mathrm{C}(13) & 1.214(12) \\ \mathrm{O}(6 \mathrm{~B})-\mathrm{C}(13 \mathrm{~B}) & 1.225(11) \\ \mathrm{O}(7)-\mathrm{C}(15) & 1.222(3) \\ \mathrm{O}(8)-\mathrm{C}(20) & 1.425(3) \\ \mathrm{O}(8)-\mathrm{C}(28) & 1.434(3) \\ \mathrm{O}(9)-\mathrm{C}(29) & 1.364(3) \\ \mathrm{O}(9)-\mathrm{C}(21) & 1.444(3) \\ \mathrm{O}(10)-\mathrm{C}(29) & 1.220(3) \\ \mathrm{N}(1)-\mathrm{C}(29) & 1.323(3) \\ \mathrm{N}(1)-\mathrm{H}(1 \mathrm{~A}) & 0.8800 \\ \mathrm{~N}(1)-\mathrm{H}(1 \mathrm{~B}) & 0.8800 \\ \mathrm{~N}(2)-\mathrm{C}(15) & 1.373(3) \\ \mathrm{N}(2)-\mathrm{C}(11) & 1.379(3) \\ \mathrm{N}(2)-\mathrm{H}(2 \mathrm{~A}) & 0.8800 \\ \mathrm{C}(1)-\mathrm{C}(2) & 1.474(3) \\ \mathrm{C}(1)-\mathrm{C}(22) & 1.496(4) \\ \mathrm{C}(1)-\mathrm{C}(21) & 1.511(4) \\ \mathrm{C}(2)-\mathrm{C}(3) & 1.506(4) \\ \mathrm{C}(2)-\mathrm{H}(2 \mathrm{~B}) & 1.0000 \\ \mathrm{C}(3)-\mathrm{C}(23) & 1.529(4) \\ \mathrm{C}(3)-\mathrm{C}(4) & 1.533(4) \\ \mathrm{C}(3)-\mathrm{H}(3) & 1.0000 \\ \mathrm{C}(4)-\mathrm{C}(5) & 1.532(3) \\ \mathrm{C}(4)-\mathrm{H}(4 \mathrm{~A}) & 1.0000 \\ \mathrm{C}(5)-\mathrm{C}(6) & 1.532(4) \\ \mathrm{C}(5)-\mathrm{H}(5) & 1.0000 \\ \mathrm{C}(6)-\mathrm{C}(7) & 1.531(4) \\ \mathrm{C}(6)-\mathrm{H}(6 \mathrm{~A}) & 0.9900 \\ \mathrm{C}(6)-\mathrm{H}(6 \mathrm{~B}) & 0.9900 \\ \mathrm{C}(7)-\mathrm{C}(25) & 1.530(4) \\ \mathrm{C}(7)-\mathrm{C}(8) & \\ \mathrm{C}(7)-\mathrm{H}(7) & \\ & \\ & 1.0000 \\ & \end{array}$




\begin{tabular}{|c|c|}
\hline$C(8)-C(9)$ & $1.500(4)$ \\
\hline $\mathrm{C}(8)-\mathrm{H}(8 \mathrm{~A})$ & 0.9900 \\
\hline $\mathrm{C}(8)-\mathrm{H}(8 \mathrm{~B})$ & 0.9900 \\
\hline$C(9)-C(14)$ & $1.352(12)$ \\
\hline$C(9)-C(14 B)$ & $1.376(11)$ \\
\hline$C(9)-C(10)$ & $1.461(3)$ \\
\hline$C(10)-C(11)$ & $1.515(3)$ \\
\hline$C(11)-C(12)$ & $1.343(3)$ \\
\hline$C(12)-C(13 B)$ & $1.467(11)$ \\
\hline$C(12)-C(13)$ & $1.470(12)$ \\
\hline $\mathrm{C}(12)-\mathrm{H}(12 \mathrm{~B})$ & 0.9500 \\
\hline$C(12)-H(12 A)$ & 0.9500 \\
\hline$C(13)-C(14)$ & $1.490(17)$ \\
\hline$C(13 B)-C(14 B)$ & $1.503(16)$ \\
\hline$C(15)-C(16)$ & $1.494(3)$ \\
\hline$C(16)-C(17)$ & $1.337(4)$ \\
\hline$C(16)-C(27)$ & $1.496(4)$ \\
\hline$C(17)-C(18)$ & $1.450(3)$ \\
\hline $\mathrm{C}(17)-\mathrm{H}(17)$ & 0.9500 \\
\hline$C(18)-C(19)$ & $1.343(4)$ \\
\hline $\mathrm{C}(18)-\mathrm{H}(18)$ & 0.9500 \\
\hline$C(19)-C(20)$ & $1.495(4)$ \\
\hline $\mathrm{C}(19)-\mathrm{H}(19)$ & 0.9500 \\
\hline$C(20)-C(21)$ & $1.525(3)$ \\
\hline $\mathrm{C}(20)-\mathrm{H}(20)$ & 1.0000 \\
\hline $\mathrm{C}(21)-\mathrm{H}(21)$ & 1.0000 \\
\hline $\mathrm{C}(22)-\mathrm{H}(22 \mathrm{~A})$ & 0.9800 \\
\hline $\mathrm{C}(22)-\mathrm{H}(22 \mathrm{~B})$ & 0.9800 \\
\hline $\mathrm{C}(22)-\mathrm{H}(22 \mathrm{C})$ & 0.9800 \\
\hline $\mathrm{C}(23)-\mathrm{H}(23 \mathrm{~A})$ & 0.9800 \\
\hline $\mathrm{C}(23)-\mathrm{H}(23 \mathrm{~B})$ & 0.9800 \\
\hline $\mathrm{C}(23)-\mathrm{H}(23 \mathrm{C})$ & 0.9800 \\
\hline $\mathrm{C}(24)-\mathrm{H}(24 \mathrm{~A})$ & 0.9800 \\
\hline $\mathrm{C}(24)-\mathrm{H}(24 \mathrm{~B})$ & 0.9800 \\
\hline $\mathrm{C}(24)-\mathrm{H}(24 \mathrm{C})$ & 0.9800 \\
\hline$C(25)-H(25 A)$ & 0.9800 \\
\hline
\end{tabular}




\begin{tabular}{|c|c|}
\hline $\mathrm{C}(25)-\mathrm{H}(25 \mathrm{~B})$ & 0.9800 \\
\hline $\mathrm{C}(25)-\mathrm{H}(25 \mathrm{C})$ & 0.9800 \\
\hline $\mathrm{C}(26)-\mathrm{H}(26 \mathrm{~A})$ & 0.9800 \\
\hline $\mathrm{C}(26)-\mathrm{H}(26 \mathrm{~B})$ & 0.9800 \\
\hline $\mathrm{C}(26)-\mathrm{H}(26 \mathrm{C})$ & 0.9800 \\
\hline $\mathrm{C}(26)-\mathrm{H}(26 \mathrm{D})$ & 0.9800 \\
\hline $\mathrm{C}(26)-\mathrm{H}(26 \mathrm{E})$ & 0.9800 \\
\hline $\mathrm{C}(26)-\mathrm{H}(26 \mathrm{~F})$ & 0.9800 \\
\hline $\mathrm{C}(27)-\mathrm{H}(27 \mathrm{~A})$ & 0.9800 \\
\hline $\mathrm{C}(27)-\mathrm{H}(27 \mathrm{~B})$ & 0.9800 \\
\hline $\mathrm{C}(27)-\mathrm{H}(27 \mathrm{C})$ & 0.9800 \\
\hline $\mathrm{C}(28)-\mathrm{H}(28 \mathrm{~A})$ & 0.9800 \\
\hline $\mathrm{C}(28)-\mathrm{H}(28 \mathrm{~B})$ & 0.9800 \\
\hline $\mathrm{C}(28)-\mathrm{H}(28 \mathrm{C})$ & 0.9800 \\
\hline $\mathrm{O}(11)-\mathrm{C}(31)$ & $1.452(3)$ \\
\hline $\mathrm{O}(11)-\mathrm{C}(30)$ & $1.463(3)$ \\
\hline $\mathrm{O}(12)-\mathrm{C}(33)$ & $1.435(3)$ \\
\hline $\mathrm{O}(12)-\mathrm{H}(12)$ & 0.8400 \\
\hline $\mathrm{O}(13)-\mathrm{C}(53)$ & $1.414(4)$ \\
\hline $\mathrm{O}(13)-\mathrm{C}(34)$ & $1.435(3)$ \\
\hline $\mathrm{O}(13 \mathrm{~B})-\mathrm{C}(43 \mathrm{~B})$ & $1.369(15)$ \\
\hline $\mathrm{O}(13 \mathrm{~B})-\mathrm{C}(55 \mathrm{~B})$ & $1.445(14)$ \\
\hline $\mathrm{O}(14)-\mathrm{C}(43)$ & $1.324(11)$ \\
\hline $\mathrm{O}(14)-\mathrm{C}(55)$ & $1.437(11)$ \\
\hline $\mathrm{O}(14 \mathrm{~B})-\mathrm{C}(42 \mathrm{~B})$ & $1.248(14)$ \\
\hline $\mathrm{O}(15)-\mathrm{C}(42)$ & $1.229(10)$ \\
\hline $\mathrm{O}(16)-\mathrm{C}(39)$ & $1.232(3)$ \\
\hline $\mathrm{O}(17)-\mathrm{C}(44)$ & $1.222(3)$ \\
\hline $\mathrm{O}(18)-\mathrm{C}(49)$ & $1.421(3)$ \\
\hline $\mathrm{O}(18)-\mathrm{C}(57)$ & $1.428(3)$ \\
\hline $\mathrm{O}(19)-\mathrm{C}(58)$ & $1.375(3)$ \\
\hline $\mathrm{O}(19)-\mathrm{C}(50)$ & $1.451(3)$ \\
\hline $\mathrm{O}(20)-\mathrm{C}(58)$ & $1.217(3)$ \\
\hline$N(3)-C(58)$ & $1.329(3)$ \\
\hline $\mathrm{N}(3)-\mathrm{H}(3 \mathrm{~A})$ & 0.8800 \\
\hline $\mathrm{N}(3)-\mathrm{H}(3 \mathrm{~B})$ & 0.8800 \\
\hline
\end{tabular}




$\begin{array}{ll}\mathrm{N}(4)-\mathrm{C}(44) & 1.376(3) \\ \mathrm{N}(4)-\mathrm{C}(40) & 1.378(3) \\ \mathrm{N}(4)-\mathrm{H}(4) & 0.8800 \\ \mathrm{C}(30)-\mathrm{C}(31) & 1.478(3) \\ \mathrm{C}(30)-\mathrm{C}(50) & 1.498(4) \\ \mathrm{C}(30)-\mathrm{C}(51) & 1.501(4) \\ \mathrm{C}(31)-\mathrm{C}(32) & 1.509(4) \\ \mathrm{C}(31)-\mathrm{H}(31) & 1.0000 \\ \mathrm{C}(32)-\mathrm{C}(52) & 1.531(4) \\ \mathrm{C}(32)-\mathrm{C}(33) & 1.531(4) \\ \mathrm{C}(32)-\mathrm{H}(32) & 1.0000 \\ \mathrm{C}(33)-\mathrm{C}(34) & 1.534(4) \\ \mathrm{C}(33)-\mathrm{H}(33) & 1.0000 \\ \mathrm{C}(34)-\mathrm{C}(35) & 1.525(4) \\ \mathrm{C}(34)-\mathrm{H}(34) & 1.0000 \\ \mathrm{C}(35)-\mathrm{C}(36) & 1.533(4) \\ \mathrm{C}(35)-\mathrm{H}(35 \mathrm{~A}) & 0.9900 \\ \mathrm{C}(35)-\mathrm{H}(35 \mathrm{~B}) & 0.9900 \\ \mathrm{C}(36)-\mathrm{C}(37) & 1.537(4) \\ \mathrm{C}(36)-\mathrm{C}(54) & 1.542(3) \\ \mathrm{C}(36)-\mathrm{H}(36) & 1.0000 \\ \mathrm{C}(37)-\mathrm{C}(38) & 1.505(3) \\ \mathrm{C}(37)-\mathrm{H}(37 \mathrm{~A}) & 0.9900 \\ \mathrm{C}(37)-\mathrm{H}(37 \mathrm{~B}) & 0.9900 \\ \mathrm{C}(38)-\mathrm{C}(43) & 1.337(11) \\ \mathrm{C}(38)-\mathrm{C}(43 \mathrm{~B}) & 1.392(14) \\ \mathrm{C}(38)-\mathrm{C}(39) & 1.462(3) \\ \mathrm{C}(39)-\mathrm{C}(40) & 1.501(3) \\ \mathrm{C}(40)-\mathrm{C}(41) & 1.342(3) \\ \mathrm{C}(41)-\mathrm{C}(42) & 1.430(11) \\ \mathrm{C}(41)-\mathrm{C}(42 \mathrm{~B}) & 1.503(13) \\ \mathrm{C}(41)-\mathrm{H}(41 \mathrm{~A}) & 0.9500 \\ \mathrm{C}(41)-\mathrm{H}(41) & 0.9500 \\ \mathrm{C}(42)-\mathrm{C}(43) & 1.482(15) \\ \mathrm{C}(42 \mathrm{~B})-\mathrm{C}(43 \mathrm{~B}) & 1.508(19) \\ \mathrm{C}(44)-\mathrm{C}(45) & 1.489(3) \\ & \\ & \end{array}$




$\begin{array}{ll}\mathrm{C}(45)-\mathrm{C}(46) & 1.344(4) \\ \mathrm{C}(45)-\mathrm{C}(56) & 1.501(4) \\ \mathrm{C}(46)-\mathrm{C}(47) & 1.452(4) \\ \mathrm{C}(46)-\mathrm{H}(46) & 0.9500 \\ \mathrm{C}(47)-\mathrm{C}(48) & 1.341(4) \\ \mathrm{C}(47)-\mathrm{H}(47) & 0.9500 \\ \mathrm{C}(48)-\mathrm{C}(49) & 1.498(4) \\ \mathrm{C}(48)-\mathrm{H}(48) & 0.9500 \\ \mathrm{C}(49)-\mathrm{C}(50) & 1.526(3) \\ \mathrm{C}(49)-\mathrm{H}(49) & 1.0000 \\ \mathrm{C}(50)-\mathrm{H}(50) & 1.0000 \\ \mathrm{C}(51)-\mathrm{H}(51 \mathrm{~A}) & 0.9800 \\ \mathrm{C}(51)-\mathrm{H}(51 \mathrm{~B}) & 0.9800 \\ \mathrm{C}(51)-\mathrm{H}(51 \mathrm{C}) & 0.9800 \\ \mathrm{C}(52)-\mathrm{H}(52 \mathrm{~A}) & 0.9800 \\ \mathrm{C}(52)-\mathrm{H}(52 \mathrm{~B}) & 0.9800 \\ \mathrm{C}(52)-\mathrm{H}(52 \mathrm{C}) & 0.9800 \\ \mathrm{C}(53)-\mathrm{H}(53 \mathrm{~A}) & 0.9800 \\ \mathrm{C}(53)-\mathrm{H}(53 \mathrm{~B}) & 0.9800 \\ \mathrm{C}(53)-\mathrm{H}(53 \mathrm{C}) & 0.9800 \\ \mathrm{C}(54)-\mathrm{H}(54 \mathrm{~A}) & 0.9800 \\ \mathrm{C}(54)-\mathrm{H}(54 \mathrm{~B}) & 0.9800 \\ \mathrm{C}(54)-\mathrm{H}(54 \mathrm{C}) & 0.9800 \\ \mathrm{C}(55)-\mathrm{H}(55 \mathrm{~A}) & 0.9800 \\ \mathrm{C}(55)-\mathrm{H}(55 \mathrm{~B}) & 0.9800 \\ \mathrm{C}(55)-\mathrm{H}(55 \mathrm{C}) & 0.9800 \\ \mathrm{C}(55 \mathrm{~B})-\mathrm{H}(55 \mathrm{D}) & 0.9800 \\ \mathrm{C}(55 \mathrm{~B})-\mathrm{H}(55 \mathrm{E}) & 0.9800 \\ \mathrm{C}(55 \mathrm{~B})-\mathrm{H}(55 \mathrm{~F}) & 0.9800 \\ \mathrm{C}(56)-\mathrm{H}(56 \mathrm{~A}) & 0.9800 \\ \mathrm{C}(56)-\mathrm{H}(56 \mathrm{~B}) & 0.9800 \\ \mathrm{C}(56)-\mathrm{H}(56 \mathrm{C}) & 0.9800 \\ \mathrm{C}(57)-\mathrm{H}(57 \mathrm{~A}) & 0.9800 \\ \mathrm{C}(57)-\mathrm{H}(57 \mathrm{~B}) & 0.9800 \\ \mathrm{C}(57)-\mathrm{H}(57 \mathrm{C}) & 0.9800 \\ & \\ & \end{array}$




\begin{tabular}{ll}
$\mathrm{C}(1)-\mathrm{O}(1)-\mathrm{C}(2)$ & \multicolumn{1}{l}{$60.95(15)$} \\
$\mathrm{C}(4)-\mathrm{O}(2)-\mathrm{H}(2)$ & 109.5 \\
$\mathrm{C}(24)-\mathrm{O}(3)-\mathrm{C}(5)$ & $114.0(2)$ \\
$\mathrm{C}(14)-\mathrm{O}(5)-\mathrm{C}(26)$ & $120.7(11)$ \\
$\mathrm{C}(26)-\mathrm{O}(5 \mathrm{~B})-\mathrm{C}(14 \mathrm{~B})$ & $132.3(14)$ \\
$\mathrm{C}(20)-\mathrm{O}(8)-\mathrm{C}(28)$ & $113.71(18)$ \\
$\mathrm{C}(29)-\mathrm{O}(9)-\mathrm{C}(21)$ & $116.52(19)$ \\
$\mathrm{C}(29)-\mathrm{N}(1)-\mathrm{H}(1 \mathrm{~A})$ & 120.0 \\
$\mathrm{C}(29)-\mathrm{N}(1)-\mathrm{H}(1 \mathrm{~B})$ & 120.0 \\
$\mathrm{H}(1 \mathrm{~A})-\mathrm{N}(1)-\mathrm{H}(1 \mathrm{~B})$ & 120.0 \\
$\mathrm{C}(15)-\mathrm{N}(2)-\mathrm{C}(11)$ & $128.4(2)$ \\
$\mathrm{C}(15)-\mathrm{N}(2)-\mathrm{H}(2 \mathrm{~A})$ & 115.8 \\
$\mathrm{C}(11)-\mathrm{N}(2)-\mathrm{H}(2 \mathrm{~A})$ & 115.8 \\
$\mathrm{O}(1)-\mathrm{C}(1)-\mathrm{C}(2)$ & $59.60(15)$ \\
$\mathrm{O}(1)-\mathrm{C}(1)-\mathrm{C}(22)$ & $115.1(2)$ \\
$\mathrm{C}(2)-\mathrm{C}(1)-\mathrm{C}(22)$ & $123.1(2)$ \\
$\mathrm{O}(1)-\mathrm{C}(1)-\mathrm{C}(21)$ & $111.8(2)$ \\
$\mathrm{C}(2)-\mathrm{C}(1)-\mathrm{C}(21)$ & $114.5(2)$ \\
$\mathrm{C}(22)-\mathrm{C}(1)-\mathrm{C}(21)$ & $118.1(2)$ \\
$\mathrm{O}(1)-\mathrm{C}(2)-\mathrm{C}(1)$ & $59.45(15)$ \\
$\mathrm{O}(1)-\mathrm{C}(2)-\mathrm{C}(3)$ & $115.99(19)$ \\
$\mathrm{C}(1)-\mathrm{C}(2)-\mathrm{C}(3)$ & $126.2(2)$ \\
$\mathrm{O}(1)-\mathrm{C}(2)-\mathrm{H}(2 \mathrm{~B})$ & 114.4 \\
$\mathrm{C}(1)-\mathrm{C}(2)-\mathrm{H}(2 \mathrm{~B})$ & 114.4 \\
$\mathrm{C}(3)-\mathrm{C}(2)-\mathrm{H}(2 \mathrm{~B})$ & 114.4 \\
$\mathrm{C}(2)-\mathrm{C}(3)-\mathrm{C}(23)$ & $107.7(2)$ \\
$\mathrm{C}(2)-\mathrm{C}(3)-\mathrm{C}(4)$ & $111.1(2)$ \\
$\mathrm{C}(23)-\mathrm{C}(3)-\mathrm{C}(4)$ & $114.6(2)$ \\
$\mathrm{C}(2)-\mathrm{C}(3)-\mathrm{H}(3)$ & 107.7 \\
$\mathrm{C}(23)-\mathrm{C}(3)-\mathrm{H}(3)$ & 107.7 \\
$\mathrm{C}(4)-\mathrm{C}(3)-\mathrm{H}(3)$ & 107.7 \\
$\mathrm{O}(2)-\mathrm{C}(4)-\mathrm{C}(5)$ & $110.76(18)$ \\
$\mathrm{O}(2)-\mathrm{C}(4)-\mathrm{C}(3)$ & $112.1(2)$ \\
$\mathrm{C}(5)-\mathrm{C}(4)-\mathrm{C}(3)$ & $111.6(2)$ \\
$\mathrm{O}(2)-\mathrm{C}(4)-\mathrm{H}(4 \mathrm{~A})$ & 107.4 \\
$\mathrm{C}(5)-\mathrm{C}(4)-\mathrm{H}(4 \mathrm{~A})$ & 107.4 \\
& \\
&
\end{tabular}




$\begin{array}{ll}\mathrm{C}(3)-\mathrm{C}(4)-\mathrm{H}(4 \mathrm{~A}) & 107.4 \\ \mathrm{O}(3)-\mathrm{C}(5)-\mathrm{C}(4) & 105.46(19) \\ \mathrm{O}(3)-\mathrm{C}(5)-\mathrm{C}(6) & 113.9(2) \\ \mathrm{C}(4)-\mathrm{C}(5)-\mathrm{C}(6) & 114.6(2) \\ \mathrm{O}(3)-\mathrm{C}(5)-\mathrm{H}(5) & 107.5 \\ \mathrm{C}(4)-\mathrm{C}(5)-\mathrm{H}(5) & 107.5 \\ \mathrm{C}(6)-\mathrm{C}(5)-\mathrm{H}(5) & 107.5 \\ \mathrm{C}(7)-\mathrm{C}(6)-\mathrm{C}(5) & 116.9(2) \\ \mathrm{C}(7)-\mathrm{C}(6)-\mathrm{H}(6 \mathrm{~A}) & 108.1 \\ \mathrm{C}(5)-\mathrm{C}(6)-\mathrm{H}(6 \mathrm{~A}) & 108.1 \\ \mathrm{C}(7)-\mathrm{C}(6)-\mathrm{H}(6 \mathrm{~B}) & 108.1 \\ \mathrm{C}(5)-\mathrm{C}(6)-\mathrm{H}(6 \mathrm{~B}) & 108.1 \\ \mathrm{H}(6 \mathrm{~A})-\mathrm{C}(6)-\mathrm{H}(6 \mathrm{~B}) & 107.3 \\ \mathrm{C}(25)-\mathrm{C}(7)-\mathrm{C}(6) & 112.3(2) \\ \mathrm{C}(25)-\mathrm{C}(7)-\mathrm{C}(8) & 108.5(2) \\ \mathrm{C}(6)-\mathrm{C}(7)-\mathrm{C}(8) & 110.5(2) \\ \mathrm{C}(25)-\mathrm{C}(7)-\mathrm{H}(7) & 108.5 \\ \mathrm{C}(6)-\mathrm{C}(7)-\mathrm{H}(7) & 108.5 \\ \mathrm{C}(8)-\mathrm{C}(7)-\mathrm{H}(7) & 108.5 \\ \mathrm{C}(9)-\mathrm{C}(8)-\mathrm{C}(7) & 115.4(2) \\ \mathrm{C}(9)-\mathrm{C}(8)-\mathrm{H}(8 \mathrm{~A}) & 108.4 \\ \mathrm{C}(7)-\mathrm{C}(8)-\mathrm{H}(8 \mathrm{~A}) & 108.4 \\ \mathrm{C}(9)-\mathrm{C}(8)-\mathrm{H}(8 \mathrm{~B}) & 108.4 \\ \mathrm{C}(7)-\mathrm{C}(8)-\mathrm{H}(8 \mathrm{~B}) & 108.4 \\ \mathrm{H}(8 \mathrm{~A})-\mathrm{C}(8)-\mathrm{H}(8 \mathrm{~B}) & 107.5 \\ \mathrm{C}(14)-\mathrm{C}(9)-\mathrm{C}(10) & 119.7(6) \\ \mathrm{C}(14 \mathrm{~B})-\mathrm{C}(9)-\mathrm{C}(10) & 117.3(6) \\ \mathrm{C}(14)-\mathrm{C}(9)-\mathrm{C}(8) & 122.2(6) \\ \mathrm{C}(14 \mathrm{~B})-\mathrm{C}(9)-\mathrm{C}(8) & 124.4(6) \\ \mathrm{C}(10)-\mathrm{C}(9)-\mathrm{C}(8) & 117.6(2) \\ \mathrm{O}(4)-\mathrm{C}(10)-\mathrm{C}(9) & 122.9(2) \\ \mathrm{O}(4)-\mathrm{C}(10)-\mathrm{C}(11) & 117.7(2) \\ \mathrm{C}(9)-\mathrm{C}(10)-\mathrm{C}(11) & 119.4(2) \\ \mathrm{C}(12)-\mathrm{C}(11)-\mathrm{N}(2) & 128.3(2) \\ \mathrm{C}(12)-\mathrm{C}(11)-\mathrm{C}(10) & 120.8(2) \\ \mathrm{N}(2)-\mathrm{C}(11)-\mathrm{C}(10) & 110.9(2) \\ & \end{array}$




\begin{tabular}{|c|c|}
\hline$C(11)-C(12)-C(13 B)$ & $121.2(5)$ \\
\hline$C(11)-C(12)-C(13)$ & $117.8(5)$ \\
\hline$C(11)-C(12)-H(12 B)$ & 119.4 \\
\hline$C(13 B)-C(12)-H(12 B)$ & 119.4 \\
\hline $\mathrm{C}(11)-\mathrm{C}(12)-\mathrm{H}(12 \mathrm{~A})$ & 121.1 \\
\hline$C(13)-C(12)-H(12 A)$ & 121.1 \\
\hline $\mathrm{O}(6)-\mathrm{C}(13)-\mathrm{C}(12)$ & $120.4(9)$ \\
\hline $\mathrm{O}(6)-\mathrm{C}(13)-\mathrm{C}(14)$ & $118.0(10)$ \\
\hline$C(12)-C(13)-C(14)$ & $121.5(9)$ \\
\hline $\mathrm{O}(6 \mathrm{~B})-\mathrm{C}(13 \mathrm{~B})-\mathrm{C}(12)$ & $120.3(9)$ \\
\hline $\mathrm{O}(6 \mathrm{~B})-\mathrm{C}(13 \mathrm{~B})-\mathrm{C}(14 \mathrm{~B})$ & $123.2(10)$ \\
\hline$C(12)-C(13 B)-C(14 B)$ & 116.6(9) \\
\hline $\mathrm{O}(5)-\mathrm{C}(14)-\mathrm{C}(9)$ & $117.6(12)$ \\
\hline $\mathrm{O}(5)-\mathrm{C}(14)-\mathrm{C}(13)$ & $122.7(11)$ \\
\hline$C(9)-C(14)-C(13)$ & $119.5(11)$ \\
\hline$O(5 B)-C(14 B)-C(9)$ & $120.1(11)$ \\
\hline$O(5 B)-C(14 B)-C(13 B)$ & $116.0(11)$ \\
\hline$C(9)-C(14 B)-C(13 B)$ & $123.6(10)$ \\
\hline $\mathrm{O}(7)-\mathrm{C}(15)-\mathrm{N}(2)$ & $122.3(2)$ \\
\hline $\mathrm{O}(7)-\mathrm{C}(15)-\mathrm{C}(16)$ & $122.2(2)$ \\
\hline $\mathrm{N}(2)-\mathrm{C}(15)-\mathrm{C}(16)$ & $115.5(2)$ \\
\hline$C(17)-C(16)-C(15)$ & $118.8(2)$ \\
\hline$C(17)-C(16)-C(27)$ & $125.9(2)$ \\
\hline$C(15)-C(16)-C(27)$ & $115.3(2)$ \\
\hline$C(16)-C(17)-C(18)$ & $127.4(2)$ \\
\hline $\mathrm{C}(16)-\mathrm{C}(17)-\mathrm{H}(17)$ & 116.3 \\
\hline $\mathrm{C}(18)-\mathrm{C}(17)-\mathrm{H}(17)$ & 116.3 \\
\hline$C(19)-C(18)-C(17)$ & 123.6(2) \\
\hline $\mathrm{C}(19)-\mathrm{C}(18)-\mathrm{H}(18)$ & 118.2 \\
\hline $\mathrm{C}(17)-\mathrm{C}(18)-\mathrm{H}(18)$ & 118.2 \\
\hline$C(18)-C(19)-C(20)$ & $125.8(2)$ \\
\hline $\mathrm{C}(18)-\mathrm{C}(19)-\mathrm{H}(19)$ & 117.1 \\
\hline $\mathrm{C}(20)-\mathrm{C}(19)-\mathrm{H}(19)$ & 117.1 \\
\hline $\mathrm{O}(8)-\mathrm{C}(20)-\mathrm{C}(19)$ & $110.2(2)$ \\
\hline $\mathrm{O}(8)-\mathrm{C}(20)-\mathrm{C}(21)$ & $108.30(18)$ \\
\hline$C(19)-C(20)-C(21)$ & $112.5(2)$ \\
\hline
\end{tabular}




\begin{tabular}{|c|c|}
\hline $\mathrm{O}(8)-\mathrm{C}(20)-\mathrm{H}(20)$ & 108.6 \\
\hline $\mathrm{C}(19)-\mathrm{C}(20)-\mathrm{H}(20)$ & 108.6 \\
\hline $\mathrm{C}(21)-\mathrm{C}(20)-\mathrm{H}(20)$ & 108.6 \\
\hline $\mathrm{O}(9)-\mathrm{C}(21)-\mathrm{C}(1)$ & $110.05(19)$ \\
\hline $\mathrm{O}(9)-\mathrm{C}(21)-\mathrm{C}(20)$ & $107.84(19)$ \\
\hline$C(1)-C(21)-C(20)$ & $113.2(2)$ \\
\hline $\mathrm{O}(9)-\mathrm{C}(21)-\mathrm{H}(21)$ & 108.6 \\
\hline $\mathrm{C}(1)-\mathrm{C}(21)-\mathrm{H}(21)$ & 108.6 \\
\hline $\mathrm{C}(20)-\mathrm{C}(21)-\mathrm{H}(21)$ & 108.6 \\
\hline $\mathrm{C}(1)-\mathrm{C}(22)-\mathrm{H}(22 \mathrm{~A})$ & 109.5 \\
\hline $\mathrm{C}(1)-\mathrm{C}(22)-\mathrm{H}(22 \mathrm{~B})$ & 109.5 \\
\hline $\mathrm{H}(22 \mathrm{~A})-\mathrm{C}(22)-\mathrm{H}(22 \mathrm{~B})$ & 109.5 \\
\hline $\mathrm{C}(1)-\mathrm{C}(22)-\mathrm{H}(22 \mathrm{C})$ & 109.5 \\
\hline $\mathrm{H}(22 \mathrm{~A})-\mathrm{C}(22)-\mathrm{H}(22 \mathrm{C})$ & 109.5 \\
\hline $\mathrm{H}(22 \mathrm{~B})-\mathrm{C}(22)-\mathrm{H}(22 \mathrm{C})$ & 109.5 \\
\hline$C(3)-C(23)-H(23 A)$ & 109.5 \\
\hline $\mathrm{C}(3)-\mathrm{C}(23)-\mathrm{H}(23 \mathrm{~B})$ & 109.5 \\
\hline$H(23 A)-C(23)-H(23 B)$ & 109.5 \\
\hline $\mathrm{C}(3)-\mathrm{C}(23)-\mathrm{H}(23 \mathrm{C})$ & 109.5 \\
\hline$H(23 A)-C(23)-H(23 C)$ & 109.5 \\
\hline $\mathrm{H}(23 \mathrm{~B})-\mathrm{C}(23)-\mathrm{H}(23 \mathrm{C})$ & 109.5 \\
\hline $\mathrm{O}(3)-\mathrm{C}(24)-\mathrm{H}(24 \mathrm{~A})$ & 109.5 \\
\hline $\mathrm{O}(3)-\mathrm{C}(24)-\mathrm{H}(24 \mathrm{~B})$ & 109.5 \\
\hline $\mathrm{H}(24 \mathrm{~A})-\mathrm{C}(24)-\mathrm{H}(24 \mathrm{~B})$ & 109.5 \\
\hline $\mathrm{O}(3)-\mathrm{C}(24)-\mathrm{H}(24 \mathrm{C})$ & 109.5 \\
\hline$H(24 A)-C(24)-H(24 C)$ & 109.5 \\
\hline $\mathrm{H}(24 \mathrm{~B})-\mathrm{C}(24)-\mathrm{H}(24 \mathrm{C})$ & 109.5 \\
\hline$C(7)-C(25)-H(25 A)$ & 109.5 \\
\hline $\mathrm{C}(7)-\mathrm{C}(25)-\mathrm{H}(25 \mathrm{~B})$ & 109.5 \\
\hline$H(25 A)-C(25)-H(25 B)$ & 109.5 \\
\hline $\mathrm{C}(7)-\mathrm{C}(25)-\mathrm{H}(25 \mathrm{C})$ & 109.5 \\
\hline$H(25 A)-C(25)-H(25 C)$ & 109.5 \\
\hline$H(25 B)-C(25)-H(25 C)$ & 109.5 \\
\hline $\mathrm{O}(5)-\mathrm{C}(26)-\mathrm{H}(26 \mathrm{~A})$ & 109.5 \\
\hline $\mathrm{O}(5)-\mathrm{C}(26)-\mathrm{H}(26 \mathrm{~B})$ & 109.5 \\
\hline$H(26 A)-C(26)-H(26 B)$ & 109.5 \\
\hline
\end{tabular}




\begin{tabular}{|c|c|}
\hline $\mathrm{O}(5)-\mathrm{C}(26)-\mathrm{H}(26 \mathrm{C})$ & 109.5 \\
\hline$H(26 A)-C(26)-H(26 C)$ & 109.5 \\
\hline $\mathrm{H}(26 \mathrm{~B})-\mathrm{C}(26)-\mathrm{H}(26 \mathrm{C})$ & 109.5 \\
\hline $\mathrm{O}(5 \mathrm{~B})-\mathrm{C}(26)-\mathrm{H}(26 \mathrm{D})$ & 109.5 \\
\hline $\mathrm{O}(5 \mathrm{~B})-\mathrm{C}(26)-\mathrm{H}(26 \mathrm{E})$ & 109.5 \\
\hline$H(26 D)-C(26)-H(26 E)$ & 109.5 \\
\hline $\mathrm{O}(5 \mathrm{~B})-\mathrm{C}(26)-\mathrm{H}(26 \mathrm{~F})$ & 109.5 \\
\hline $\mathrm{H}(26 \mathrm{D})-\mathrm{C}(26)-\mathrm{H}(26 \mathrm{~F})$ & 109.5 \\
\hline $\mathrm{H}(26 \mathrm{E})-\mathrm{C}(26)-\mathrm{H}(26 \mathrm{~F})$ & 109.5 \\
\hline$C(16)-C(27)-H(27 A)$ & 109.5 \\
\hline$C(16)-C(27)-H(27 B)$ & 109.5 \\
\hline $\mathrm{H}(27 \mathrm{~A})-\mathrm{C}(27)-\mathrm{H}(27 \mathrm{~B})$ & 109.5 \\
\hline $\mathrm{C}(16)-\mathrm{C}(27)-\mathrm{H}(27 \mathrm{C})$ & 109.5 \\
\hline $\mathrm{H}(27 \mathrm{~A})-\mathrm{C}(27)-\mathrm{H}(27 \mathrm{C})$ & 109.5 \\
\hline $\mathrm{H}(27 \mathrm{~B})-\mathrm{C}(27)-\mathrm{H}(27 \mathrm{C})$ & 109.5 \\
\hline $\mathrm{O}(8)-\mathrm{C}(28)-\mathrm{H}(28 \mathrm{~A})$ & 109.5 \\
\hline $\mathrm{O}(8)-\mathrm{C}(28)-\mathrm{H}(28 \mathrm{~B})$ & 109.5 \\
\hline $\mathrm{H}(28 \mathrm{~A})-\mathrm{C}(28)-\mathrm{H}(28 \mathrm{~B})$ & 109.5 \\
\hline $\mathrm{O}(8)-\mathrm{C}(28)-\mathrm{H}(28 \mathrm{C})$ & 109.5 \\
\hline$H(28 A)-C(28)-H(28 C)$ & 109.5 \\
\hline$H(28 B)-C(28)-H(28 C)$ & 109.5 \\
\hline $\mathrm{O}(10)-\mathrm{C}(29)-\mathrm{N}(1)$ & $126.8(2)$ \\
\hline $\mathrm{O}(10)-\mathrm{C}(29)-\mathrm{O}(9)$ & $122.8(2)$ \\
\hline $\mathrm{N}(1)-\mathrm{C}(29)-\mathrm{O}(9)$ & $110.4(2)$ \\
\hline$C(31)-O(11)-C(30)$ & $60.93(16)$ \\
\hline $\mathrm{C}(33)-\mathrm{O}(12)-\mathrm{H}(12)$ & 109.5 \\
\hline$C(53)-O(13)-C(34)$ & $113.1(2)$ \\
\hline$C(43 B)-O(13 B)-C(55 B)$ & $119.7(13)$ \\
\hline $\mathrm{C}(43)-\mathrm{O}(14)-\mathrm{C}(55)$ & $122.8(8)$ \\
\hline $\mathrm{C}(49)-\mathrm{O}(18)-\mathrm{C}(57)$ & $113.76(18)$ \\
\hline $\mathrm{C}(58)-\mathrm{O}(19)-\mathrm{C}(50)$ & $115.30(19)$ \\
\hline $\mathrm{C}(58)-\mathrm{N}(3)-\mathrm{H}(3 \mathrm{~A})$ & 120.0 \\
\hline $\mathrm{C}(58)-\mathrm{N}(3)-\mathrm{H}(3 \mathrm{~B})$ & 120.0 \\
\hline$H(3 A)-N(3)-H(3 B)$ & 120.0 \\
\hline$C(44)-N(4)-C(40)$ & 129.2(2) \\
\hline $\mathrm{C}(44)-\mathrm{N}(4)-\mathrm{H}(4)$ & 115.4 \\
\hline
\end{tabular}




\begin{tabular}{ll}
$\mathrm{C}(40)-\mathrm{N}(4)-\mathrm{H}(4)$ & 115.4 \\
$\mathrm{O}(11)-\mathrm{C}(30)-\mathrm{C}(31)$ & \multicolumn{1}{|c}{$59.15(16)$} \\
$\mathrm{O}(11)-\mathrm{C}(30)-\mathrm{C}(50)$ & $110.9(2)$ \\
$\mathrm{C}(31)-\mathrm{C}(30)-\mathrm{C}(50)$ & $115.1(2)$ \\
$\mathrm{O}(11)-\mathrm{C}(30)-\mathrm{C}(51)$ & $114.6(2)$ \\
$\mathrm{C}(31)-\mathrm{C}(30)-\mathrm{C}(51)$ & $122.7(3)$ \\
$\mathrm{C}(50)-\mathrm{C}(30)-\mathrm{C}(51)$ & $118.7(2)$ \\
$\mathrm{O}(11)-\mathrm{C}(31)-\mathrm{C}(30)$ & $59.92(15)$ \\
$\mathrm{O}(11)-\mathrm{C}(31)-\mathrm{C}(32)$ & $116.3(2)$ \\
$\mathrm{C}(30)-\mathrm{C}(31)-\mathrm{C}(32)$ & $125.9(2)$ \\
$\mathrm{O}(11)-\mathrm{C}(31)-\mathrm{H}(31)$ & 114.4 \\
$\mathrm{C}(30)-\mathrm{C}(31)-\mathrm{H}(31)$ & 114.4 \\
$\mathrm{C}(32)-\mathrm{C}(31)-\mathrm{H}(31)$ & 114.4 \\
$\mathrm{C}(31)-\mathrm{C}(32)-\mathrm{C}(52)$ & $108.1(2)$ \\
$\mathrm{C}(31)-\mathrm{C}(32)-\mathrm{C}(33)$ & $109.9(2)$ \\
$\mathrm{C}(52)-\mathrm{C}(32)-\mathrm{C}(33)$ & $114.5(2)$ \\
$\mathrm{C}(31)-\mathrm{C}(32)-\mathrm{H}(32)$ & 108.1 \\
$\mathrm{C}(52)-\mathrm{C}(32)-\mathrm{H}(32)$ & 108.1 \\
$\mathrm{C}(33)-\mathrm{C}(32)-\mathrm{H}(32)$ & 108.1 \\
$\mathrm{O}(12)-\mathrm{C}(33)-\mathrm{C}(32)$ & $111.7(2)$ \\
$\mathrm{O}(12)-\mathrm{C}(33)-\mathrm{C}(34)$ & $109.81(19)$ \\
$\mathrm{C}(32)-\mathrm{C}(33)-\mathrm{C}(34)$ & $112.9(2)$ \\
$\mathrm{O}(12)-\mathrm{C}(33)-\mathrm{H}(33)$ & 107.4 \\
$\mathrm{C}(32)-\mathrm{C}(33)-\mathrm{H}(33)$ & 107.4 \\
$\mathrm{C}(34)-\mathrm{C}(33)-\mathrm{H}(33)$ & 107.4 \\
$\mathrm{O}(13)-\mathrm{C}(34)-\mathrm{C}(35)$ & $114.6(2)$ \\
$\mathrm{O}(13)-\mathrm{C}(34)-\mathrm{C}(33)$ & $104.6(2)$ \\
$\mathrm{C}(35)-\mathrm{C}(34)-\mathrm{C}(33)$ & $114.0(2)$ \\
$\mathrm{O}(13)-\mathrm{C}(34)-\mathrm{H}(34)$ & 107.8 \\
$\mathrm{C}(35)-\mathrm{C}(34)-\mathrm{H}(34)$ & 107.8 \\
$\mathrm{C}(33)-\mathrm{C}(34)-\mathrm{H}(34)$ & 107.8 \\
$\mathrm{C}(34)-\mathrm{C}(35)-\mathrm{C}(36)$ & $116.7(2)$ \\
$\mathrm{C}(34)-\mathrm{C}(35)-\mathrm{H}(35 \mathrm{~A})$ & 108.1 \\
$\mathrm{C}(36)-\mathrm{C}(35)-\mathrm{H}(35 \mathrm{~A})$ & 108.1 \\
$\mathrm{C}(34)-\mathrm{C}(35)-\mathrm{H}(35 \mathrm{~B})$ & 108.1 \\
$\mathrm{C}(36)-\mathrm{C}(35)-\mathrm{H}(35 \mathrm{~B})$ & 108.1 \\
& \\
&
\end{tabular}




$\begin{array}{ll}\mathrm{H}(35 \mathrm{~A})-\mathrm{C}(35)-\mathrm{H}(35 \mathrm{~B}) & 107.3 \\ \mathrm{C}(35)-\mathrm{C}(36)-\mathrm{C}(37) & 111.5(2) \\ \mathrm{C}(35)-\mathrm{C}(36)-\mathrm{C}(54) & 111.2(2) \\ \mathrm{C}(37)-\mathrm{C}(36)-\mathrm{C}(54) & 108.7(2) \\ \mathrm{C}(35)-\mathrm{C}(36)-\mathrm{H}(36) & 108.4 \\ \mathrm{C}(37)-\mathrm{C}(36)-\mathrm{H}(36) & 108.4 \\ \mathrm{C}(54)-\mathrm{C}(36)-\mathrm{H}(36) & 108.4 \\ \mathrm{C}(38)-\mathrm{C}(37)-\mathrm{C}(36) & 114.4(2) \\ \mathrm{C}(38)-\mathrm{C}(37)-\mathrm{H}(37 \mathrm{~A}) & 108.6 \\ \mathrm{C}(36)-\mathrm{C}(37)-\mathrm{H}(37 \mathrm{~A}) & 108.6 \\ \mathrm{C}(38)-\mathrm{C}(37)-\mathrm{H}(37 \mathrm{~B}) & 108.6 \\ \mathrm{C}(36)-\mathrm{C}(37)-\mathrm{H}(37 \mathrm{~B}) & 108.6 \\ \mathrm{H}(37 \mathrm{~A})-\mathrm{C}(37)-\mathrm{H}(37 \mathrm{~B}) & 107.6 \\ \mathrm{C}(43)-\mathrm{C}(38)-\mathrm{C}(39) & 118.0(5) \\ \mathrm{C}(43 \mathrm{~B})-\mathrm{C}(38)-\mathrm{C}(39) & 118.6(7) \\ \mathrm{C}(43)-\mathrm{C}(38)-\mathrm{C}(37) & 123.7(5) \\ \mathrm{C}(43 \mathrm{~B})-\mathrm{C}(38)-\mathrm{C}(37) & 122.8(7) \\ \mathrm{C}(39)-\mathrm{C}(38)-\mathrm{C}(37) & 117.9(2) \\ \mathrm{O}(16)-\mathrm{C}(39)-\mathrm{C}(38) & 122.4(2) \\ \mathrm{O}(16)-\mathrm{C}(39)-\mathrm{C}(40) & 118.0(2) \\ \mathrm{C}(38)-\mathrm{C}(39)-\mathrm{C}(40) & 119.6(2) \\ \mathrm{C}(41)-\mathrm{C}(40)-\mathrm{N}(4) & 128.5(2) \\ \mathrm{C}(41)-\mathrm{C}(40)-\mathrm{C}(39) & 121.0(2) \\ \mathrm{N}(4)-\mathrm{C}(40)-\mathrm{C}(39) & 110.48(19) \\ \mathrm{C}(40)-\mathrm{C}(41)-\mathrm{C}(42) & 118.2(4) \\ \mathrm{C}(40)-\mathrm{C}(41)-\mathrm{C}(42 \mathrm{~B}) & 120.8(6) \\ \mathrm{C}(40)-\mathrm{C}(41)-\mathrm{H}(41 \mathrm{~A}) & 119.6 \\ \mathrm{C}(42 \mathrm{~B})-\mathrm{C}(41)-\mathrm{H}(41 \mathrm{~A}) & 119.6 \\ \mathrm{C}(40)-\mathrm{C}(41)-\mathrm{H}(41) & 120.9 \\ \mathrm{C}(42)-\mathrm{C}(41)-\mathrm{H}(41) & 120.9 \\ \mathrm{O}(15)-\mathrm{C}(42)-\mathrm{C}(41) & 118.5(8) \\ \mathrm{O}(15)-\mathrm{C}(42)-\mathrm{C}(43) & 120.3(10) \\ \mathrm{C}(41)-\mathrm{C}(42)-\mathrm{C}(43) & 121.2(8) \\ \mathrm{O}(14 \mathrm{~B})-\mathrm{C}(42 \mathrm{~B})-\mathrm{C}(41) & 122.1(12) \\ \mathrm{O}(14 \mathrm{~B})-\mathrm{C}(42 \mathrm{~B})-\mathrm{C}(43 \mathrm{~B}) & 120.8(13) \\ & 117.0(11) \\ & \end{array}$




\begin{tabular}{|c|c|}
\hline $\mathrm{O}(14)-\mathrm{C}(43)-\mathrm{C}(38)$ & $116.3(9)$ \\
\hline$O(14)-C(43)-C(42)$ & $122.4(9)$ \\
\hline$C(38)-C(43)-C(42)$ & $121.2(9)$ \\
\hline $\mathrm{O}(13 \mathrm{~B})-\mathrm{C}(43 \mathrm{~B})-\mathrm{C}(38)$ & $123.2(12)$ \\
\hline $\mathrm{O}(13 \mathrm{~B})-\mathrm{C}(43 \mathrm{~B})-\mathrm{C}(42 \mathrm{~B})$ & $115.3(12)$ \\
\hline$C(38)-C(43 B)-C(42 B)$ & $121.4(12)$ \\
\hline $\mathrm{O}(17)-\mathrm{C}(44)-\mathrm{N}(4)$ & $121.9(2)$ \\
\hline $\mathrm{O}(17)-\mathrm{C}(44)-\mathrm{C}(45)$ & $122.2(2)$ \\
\hline $\mathrm{N}(4)-\mathrm{C}(44)-\mathrm{C}(45)$ & $115.8(2)$ \\
\hline$C(46)-C(45)-C(44)$ & 119.1(2) \\
\hline$C(46)-C(45)-C(56)$ & $125.4(2)$ \\
\hline$C(44)-C(45)-C(56)$ & $115.4(2)$ \\
\hline$C(45)-C(46)-C(47)$ & $127.8(2)$ \\
\hline $\mathrm{C}(45)-\mathrm{C}(46)-\mathrm{H}(46)$ & 116.1 \\
\hline $\mathrm{C}(47)-\mathrm{C}(46)-\mathrm{H}(46)$ & 116.1 \\
\hline$C(48)-C(47)-C(46)$ & $123.5(3)$ \\
\hline $\mathrm{C}(48)-\mathrm{C}(47)-\mathrm{H}(47)$ & 118.3 \\
\hline $\mathrm{C}(46)-\mathrm{C}(47)-\mathrm{H}(47)$ & 118.3 \\
\hline$C(47)-C(48)-C(49)$ & $126.3(2)$ \\
\hline $\mathrm{C}(47)-\mathrm{C}(48)-\mathrm{H}(48)$ & 116.8 \\
\hline $\mathrm{C}(49)-\mathrm{C}(48)-\mathrm{H}(48)$ & 116.8 \\
\hline $\mathrm{O}(18)-\mathrm{C}(49)-\mathrm{C}(48)$ & $110.7(2)$ \\
\hline $\mathrm{O}(18)-\mathrm{C}(49)-\mathrm{C}(50)$ & $108.03(18)$ \\
\hline$C(48)-C(49)-C(50)$ & $111.9(2)$ \\
\hline $\mathrm{O}(18)-\mathrm{C}(49)-\mathrm{H}(49)$ & 108.7 \\
\hline $\mathrm{C}(48)-\mathrm{C}(49)-\mathrm{H}(49)$ & 108.7 \\
\hline $\mathrm{C}(50)-\mathrm{C}(49)-\mathrm{H}(49)$ & 108.7 \\
\hline$O(19)-C(50)-C(30)$ & $109.4(2)$ \\
\hline $\mathrm{O}(19)-\mathrm{C}(50)-\mathrm{C}(49)$ & $107.29(19)$ \\
\hline$C(30)-C(50)-C(49)$ & $114.1(2)$ \\
\hline $\mathrm{O}(19)-\mathrm{C}(50)-\mathrm{H}(50)$ & 108.6 \\
\hline $\mathrm{C}(30)-\mathrm{C}(50)-\mathrm{H}(50)$ & 108.6 \\
\hline $\mathrm{C}(49)-\mathrm{C}(50)-\mathrm{H}(50)$ & 108.6 \\
\hline$C(30)-C(51)-H(51 A)$ & 109.5 \\
\hline$C(30)-C(51)-H(51 B)$ & 109.5 \\
\hline$H(51 A)-C(51)-H(51 B)$ & 109.5 \\
\hline
\end{tabular}




$\begin{array}{ll}\mathrm{C}(30)-\mathrm{C}(51)-\mathrm{H}(51 \mathrm{C}) & 109.5 \\ \mathrm{H}(51 \mathrm{~A})-\mathrm{C}(51)-\mathrm{H}(51 \mathrm{C}) & 109.5 \\ \mathrm{H}(51 \mathrm{~B})-\mathrm{C}(51)-\mathrm{H}(51 \mathrm{C}) & 109.5 \\ \mathrm{C}(32)-\mathrm{C}(52)-\mathrm{H}(52 \mathrm{~A}) & 109.5 \\ \mathrm{C}(32)-\mathrm{C}(52)-\mathrm{H}(52 \mathrm{~B}) & 109.5 \\ \mathrm{H}(52 \mathrm{~A})-\mathrm{C}(52)-\mathrm{H}(52 \mathrm{~B}) & 109.5 \\ \mathrm{C}(32)-\mathrm{C}(52)-\mathrm{H}(52 \mathrm{C}) & 109.5 \\ \mathrm{H}(52 \mathrm{~A})-\mathrm{C}(52)-\mathrm{H}(52 \mathrm{C}) & 109.5 \\ \mathrm{H}(52 \mathrm{~B})-\mathrm{C}(52)-\mathrm{H}(52 \mathrm{C}) & 109.5 \\ \mathrm{O}(13)-\mathrm{C}(53)-\mathrm{H}(53 \mathrm{~A}) & 109.5 \\ \mathrm{O}(13)-\mathrm{C}(53)-\mathrm{H}(53 \mathrm{~B}) & 109.5 \\ \mathrm{H}(53 \mathrm{~A})-\mathrm{C}(53)-\mathrm{H}(53 \mathrm{~B}) & 109.5 \\ \mathrm{O}(13)-\mathrm{C}(53)-\mathrm{H}(53 \mathrm{C}) & 109.5 \\ \mathrm{H}(53 \mathrm{~A})-\mathrm{C}(53)-\mathrm{H}(53 \mathrm{C}) & 109.5 \\ \mathrm{H}(53 \mathrm{~B})-\mathrm{C}(53)-\mathrm{H}(53 \mathrm{C}) & 109.5 \\ \mathrm{C}(36)-\mathrm{C}(54)-\mathrm{H}(54 \mathrm{~A}) & 109.5 \\ \mathrm{C}(36)-\mathrm{C}(54)-\mathrm{H}(54 \mathrm{~B}) & 109.5 \\ \mathrm{H}(54 \mathrm{~A})-\mathrm{C}(54)-\mathrm{H}(54 \mathrm{~B}) & 109.5 \\ \mathrm{C}(36)-\mathrm{C}(54)-\mathrm{H}(54 \mathrm{C}) & 109.5 \\ \mathrm{H}(54 \mathrm{~A})-\mathrm{C}(54)-\mathrm{H}(54 \mathrm{C}) & 109.5 \\ \mathrm{H}(54 \mathrm{~B})-\mathrm{C}(54)-\mathrm{H}(54 \mathrm{C}) & 109.5 \\ \mathrm{O}(14)-\mathrm{C}(55)-\mathrm{H}(55 \mathrm{~A}) & 109.5 \\ \mathrm{O}(14)-\mathrm{C}(55)-\mathrm{H}(55 \mathrm{~B}) & 109.5 \\ \mathrm{H}(55 \mathrm{~A})-\mathrm{C}(55)-\mathrm{H}(55 \mathrm{~B}) & 109.5 \\ \mathrm{O}(14)-\mathrm{C}(55)-\mathrm{H}(55 \mathrm{C}) & 109.5 \\ \mathrm{H}(55 \mathrm{~A})-\mathrm{C}(55)-\mathrm{H}(55 \mathrm{C}) & 109.5 \\ \mathrm{H}(55 \mathrm{~B})-\mathrm{C}(55)-\mathrm{H}(55 \mathrm{C}) & 109.5 \\ \mathrm{O}(13 \mathrm{~B})-\mathrm{C}(55 \mathrm{~B})-\mathrm{H}(55 \mathrm{D}) & 109.5 \\ \mathrm{O}(13 \mathrm{~B})-\mathrm{C}(55 \mathrm{~B})-\mathrm{H}(55 \mathrm{E}) & 109.5 \\ \mathrm{H}(55 \mathrm{D})-\mathrm{C}(55 \mathrm{~B})-\mathrm{H}(55 \mathrm{E}) & 109.5 \\ \mathrm{O}(13 \mathrm{~B})-\mathrm{C}(55 \mathrm{~B})-\mathrm{H}(55 \mathrm{~F}) & 109.5 \\ \mathrm{H}(55 \mathrm{D})-\mathrm{C}(55 \mathrm{~B})-\mathrm{H}(55 \mathrm{~F}) & 109.5 \\ \mathrm{H}(55 \mathrm{E})-\mathrm{C}(55 \mathrm{~B})-\mathrm{H}(55 \mathrm{~F}) & 109.5 \\ \mathrm{C}(45)-\mathrm{C}(56)-\mathrm{H}(56 \mathrm{~A}) & 109.5 \\ \mathrm{C}(45)-\mathrm{C}(56)-\mathrm{H}(56 \mathrm{~B}) & 109.5 \\ \mathrm{H}(56 \mathrm{~A})-\mathrm{C}(56)-\mathrm{H}(56 \mathrm{~B}) & 109.5 \\ & \end{array}$




$\begin{array}{ll}\mathrm{C}(45)-\mathrm{C}(56)-\mathrm{H}(56 \mathrm{C}) & 109.5 \\ \mathrm{H}(56 \mathrm{~A})-\mathrm{C}(56)-\mathrm{H}(56 \mathrm{C}) & 109.5 \\ \mathrm{H}(56 \mathrm{~B})-\mathrm{C}(56)-\mathrm{H}(56 \mathrm{C}) & 109.5 \\ \mathrm{O}(18)-\mathrm{C}(57)-\mathrm{H}(57 \mathrm{~A}) & 109.5 \\ \mathrm{O}(18)-\mathrm{C}(57)-\mathrm{H}(57 \mathrm{~B}) & 109.5 \\ \mathrm{H}(57 \mathrm{~A})-\mathrm{C}(57)-\mathrm{H}(57 \mathrm{~B}) & 109.5 \\ \mathrm{O}(18)-\mathrm{C}(57)-\mathrm{H}(57 \mathrm{C}) & 109.5 \\ \mathrm{H}(57 \mathrm{~A})-\mathrm{C}(57)-\mathrm{H}(57 \mathrm{C}) & 109.5 \\ \mathrm{H}(57 \mathrm{~B})-\mathrm{C}(57)-\mathrm{H}(57 \mathrm{C}) & 109.5 \\ \mathrm{O}(20)-\mathrm{C}(58)-\mathrm{N}(3) & 127.8(2) \\ \mathrm{O}(20)-\mathrm{C}(58)-\mathrm{O}(19) & 122.7(2) \\ \mathrm{N}(3)-\mathrm{C}(58)-\mathrm{O}(19) & 109.5(2)\end{array}$

Symmetry transformations used to generate equivalent atoms:

Table S6. Anisotropic displacement parameters $\left(\AA^{2} \times 10^{3}\right)$ for $\mathbf{A}$. The anisotropic displacement factor exponent takes the form: $-2 p^{2}\left[h^{2} a^{* 2} U^{11}+\ldots+2 h k a^{*} b^{*} U^{12}\right]$

\begin{tabular}{lcccccc}
\hline & $U^{11}$ & $U^{22}$ & $U^{33}$ & $U^{23}$ & $U^{13}$ & $U^{12}$ \\
\hline $\mathrm{O}(1)$ & $53(1)$ & $26(1)$ & $31(1)$ & $-3(1)$ & $16(1)$ & $-1(1)$ \\
$\mathrm{O}(2)$ & $35(1)$ & $36(1)$ & $27(1)$ & $-1(1)$ & $5(1)$ & $-3(1)$ \\
$\mathrm{O}(3)$ & $38(1)$ & $47(1)$ & $36(1)$ & $8(1)$ & $2(1)$ & $-5(1)$ \\
$\mathrm{O}(4)$ & $37(1)$ & $40(1)$ & $34(1)$ & $7(1)$ & $12(1)$ & $2(1)$ \\
$\mathrm{O}(5)$ & $36(3)$ & $45(6)$ & $54(6)$ & $0(3)$ & $23(3)$ & $-6(3)$ \\
$\mathrm{O}(5 \mathrm{~B})$ & $37(3)$ & $61(8)$ & $56(6)$ & $0(4)$ & $19(3)$ & $-6(4)$ \\
$\mathrm{O}(6)$ & $62(3)$ & $58(5)$ & $52(4)$ & $13(4)$ & $34(3)$ & $-7(3)$ \\
$\mathrm{O}(6 \mathrm{~B})$ & $62(3)$ & $79(7)$ & $45(3)$ & $14(4)$ & $29(2)$ & $0(4)$ \\
$\mathrm{O}(7)$ & $43(1)$ & $44(1)$ & $25(1)$ & $-2(1)$ & $7(1)$ & $6(1)$ \\
$\mathrm{O}(8)$ & $52(1)$ & $24(1)$ & $32(1)$ & $1(1)$ & $22(1)$ & $3(1)$ \\
$\mathrm{O}(9)$ & $48(1)$ & $26(1)$ & $35(1)$ & $1(1)$ & $26(1)$ & $4(1)$ \\
$\mathrm{O}(10)$ & $66(1)$ & $29(1)$ & $45(1)$ & $5(1)$ & $28(1)$ & $13(1)$ \\
$\mathrm{N}(1)$ & $65(2)$ & $31(1)$ & $45(1)$ & $2(1)$ & $36(1)$ & $13(1)$ \\
$\mathrm{N}(2)$ & $34(1)$ & $42(1)$ & $24(1)$ & $1(1)$ & $10(1)$ & $3(1)$ \\
$\mathrm{C}(1)$ & $43(1)$ & $23(1)$ & $27(1)$ & $1(1)$ & $13(1)$ & $2(1)$ \\
& & & & 46 & &
\end{tabular}




\begin{tabular}{|c|c|c|c|c|c|c|}
\hline$C(2)$ & $41(1)$ & $23(1)$ & $24(1)$ & $1(1)$ & $10(1)$ & $1(1)$ \\
\hline$C(3)$ & $40(1)$ & $31(1)$ & $26(1)$ & $0(1)$ & $7(1)$ & $-5(1)$ \\
\hline$C(4)$ & $34(1)$ & $30(1)$ & $25(1)$ & $5(1)$ & $7(1)$ & $-3(1)$ \\
\hline$C(5)$ & $36(1)$ & $33(1)$ & $32(1)$ & $6(1)$ & $6(1)$ & $-2(1)$ \\
\hline$C(6)$ & $34(1)$ & $34(2)$ & $41(1)$ & $7(1)$ & $14(1)$ & $0(1)$ \\
\hline$C(7)$ & $32(1)$ & $32(1)$ & $44(1)$ & $7(1)$ & $7(1)$ & $2(1)$ \\
\hline$C(8)$ & $30(1)$ & $34(2)$ & $54(2)$ & $2(1)$ & $14(1)$ & 2(1) \\
\hline$C(9)$ & $35(1)$ & $27(1)$ & $46(1)$ & $-3(1)$ & $14(1)$ & $-1(1)$ \\
\hline$C(10)$ & $36(1)$ & $25(1)$ & $36(1)$ & $0(1)$ & $13(1)$ & 2(1) \\
\hline$C(11)$ & $38(1)$ & $25(1)$ & $31(1)$ & $-3(1)$ & $12(1)$ & $-1(1)$ \\
\hline$C(12)$ & $41(1)$ & $36(2)$ & $34(1)$ & $2(1)$ & $13(1)$ & $0(1)$ \\
\hline$C(13)$ & $53(4)$ & $30(8)$ & $41(4)$ & $1(5)$ & $26(3)$ & 2(3) \\
\hline$C(13 B)$ & $47(4)$ & $30(8)$ & $47(5)$ & $0(5)$ & $23(3)$ & $-5(3)$ \\
\hline$C(14)$ & $36(2)$ & $6(6)$ & $49(3)$ & $-11(3)$ & $18(2)$ & $-8(3)$ \\
\hline$C(14 B)$ & $36(2)$ & $6(6)$ & $49(3)$ & $-11(3)$ & $18(2)$ & $-8(3)$ \\
\hline$C(15)$ & $35(1)$ & $27(1)$ & $32(1)$ & $-1(1)$ & $9(1)$ & $1(1)$ \\
\hline$C(16)$ & $33(1)$ & $30(1)$ & $34(1)$ & $-1(1)$ & $9(1)$ & $1(1)$ \\
\hline$C(17)$ & $33(1)$ & $36(1)$ & $35(1)$ & $0(1)$ & $12(1)$ & $0(1)$ \\
\hline$C(18)$ & $33(1)$ & $42(2)$ & $41(1)$ & $-4(1)$ & $15(1)$ & $1(1)$ \\
\hline$C(19)$ & $35(1)$ & $35(1)$ & $42(1)$ & $-4(1)$ & $21(1)$ & $0(1)$ \\
\hline C(20) & $39(1)$ & $26(1)$ & $29(1)$ & $0(1)$ & $20(1)$ & $1(1)$ \\
\hline$C(21)$ & $40(1)$ & $24(1)$ & $28(1)$ & $4(1)$ & $18(1)$ & $3(1)$ \\
\hline C(22) & $48(2)$ & $37(2)$ & $35(1)$ & 11(1) & $10(1)$ & $1(1)$ \\
\hline$C(23)$ & $45(1)$ & $31(1)$ & $43(1)$ & $-3(1)$ & $14(1)$ & $-9(1)$ \\
\hline C(24) & $37(1)$ & $58(2)$ & $59(2)$ & 2(2) & $-3(1)$ & $-7(1)$ \\
\hline$C(25)$ & $36(1)$ & $42(2)$ & $61(2)$ & $12(1)$ & $-2(1)$ & $-1(1)$ \\
\hline$C(26)$ & $68(2)$ & $145(5)$ & $99(3)$ & $-38(4)$ & $51(2)$ & $-24(3)$ \\
\hline $\mathrm{C}(27)$ & $35(1)$ & 62(2) & $41(1)$ & $-9(1)$ & $8(1)$ & 1(1) \\
\hline$C(28)$ & $71(2)$ & $28(1)$ & $43(1)$ & $-1(1)$ & $27(1)$ & $8(1)$ \\
\hline C(29) & $44(1)$ & $27(1)$ & $36(1)$ & $-4(1)$ & $18(1)$ & $4(1)$ \\
\hline $\mathrm{O}(11)$ & $61(1)$ & $29(1)$ & $38(1)$ & $8(1)$ & $23(1)$ & $5(1)$ \\
\hline $\mathrm{O}(12)$ & $41(1)$ & $38(1)$ & $28(1)$ & $-2(1)$ & $5(1)$ & $6(1)$ \\
\hline $\mathrm{O}(13)$ & $50(1)$ & $38(1)$ & $31(1)$ & $-2(1)$ & $-3(1)$ & $8(1)$ \\
\hline $\mathrm{O}(13 \mathrm{~B})$ & $28(3)$ & $37(5)$ & $41(4)$ & $6(3)$ & $12(2)$ & $10(3)$ \\
\hline $\mathrm{O}(14)$ & $37(2)$ & $87(6)$ & $63(4)$ & $-15(4)$ & $9(2)$ & $15(3)$ \\
\hline \multirow[t]{2}{*}{$\mathrm{O}(14 \mathrm{~B})$} & $52(4)$ & $33(4)$ & $43(3)$ & $1(3)$ & $29(3)$ & $-6(3)$ \\
\hline & & & & 47 & & \\
\hline
\end{tabular}




$\begin{array}{lcccccc}\mathrm{O}(15) & 44(3) & 160(10) & 75(5) & -65(6) & 20(3) & 13(5) \\ \mathrm{O}(16) & 37(1) & 42(1) & 30(1) & -4(1) & 9(1) & 0(1) \\ \mathrm{O}(17) & 39(1) & 40(1) & 26(1) & 1(1) & 9(1) & -4(1) \\ \mathrm{O}(18) & 54(1) & 23(1) & 40(1) & 0(1) & 30(1) & -1(1) \\ \mathrm{O}(19) & 54(1) & 24(1) & 45(1) & 2(1) & 35(1) & -1(1) \\ \mathrm{O}(20) & 64(1) & 30(1) & 46(1) & 0(1) & 27(1) & -7(1) \\ \mathrm{N}(3) & 65(2) & 33(1) & 47(1) & 3(1) & 37(1) & -9(1) \\ \mathrm{N}(4) & 37(1) & 45(1) & 23(1) & -2(1) & 11(1) & -4(1) \\ \mathrm{C}(30) & 55(2) & 26(1) & 31(1) & 2(1) & 20(1) & 4(1) \\ \mathrm{C}(31) & 48(1) & 28(1) & 30(1) & 1(1) & 16(1) & 4(1) \\ \mathrm{C}(32) & 50(1) & 31(1) & 29(1) & -4(1) & 9(1) & 3(1) \\ \mathrm{C}(33) & 43(1) & 27(1) & 27(1) & -5(1) & 5(1) & 6(1) \\ \mathrm{C}(34) & 41(1) & 30(1) & 30(1) & -6(1) & 3(1) & 5(1) \\ \mathrm{C}(35) & 35(1) & 31(1) & 36(1) & -6(1) & 4(1) & 6(1) \\ \mathrm{C}(36) & 38(1) & 30(1) & 32(1) & -7(1) & -1(1) & 3(1) \\ \mathrm{C}(37) & 35(1) & 34(1) & 40(1) & -2(1) & 2(1) & 2(1) \\ \mathrm{C}(38) & 36(1) & 30(1) & 37(1) & -2(1) & 6(1) & 3(1) \\ \mathrm{C}(39) & 36(1) & 27(1) & 28(1) & 1(1) & 9(1) & 1(1) \\ \mathrm{C}(40) & 35(1) & 26(1) & 28(1) & 1(1) & 9(1) & -4(1) \\ \mathrm{C}(41) & 38(1) & 33(1) & 31(1) & -1(1) & 10(1) & 4(1) \\ \mathrm{C}(42) & 55(5) & 61(10) & 51(5) & -24(6) & 21(4) & -3(4) \\ \mathrm{C}(42 \mathrm{~B}) & 28(5) & 27(6) & 36(5) & 7(4) & 13(4) & 9(4) \\ \mathrm{C}(43) & 39(4) & 26(7) & 53(5) & 1(4) & 10(3) & 4(3) \\ \mathrm{C}(43 \mathrm{~B}) & 31(5) & 13(8) & 34(5) & 10(4) & 12(3) & 0(4) \\ \mathrm{C}(44) & 35(1) & 26(1) & 28(1) & 3(1) & 8(1) & -1(1) \\ \mathrm{C}(45) & 37(1) & 32(1) & 31(1) & -1(1) & 8(1) & -1(1) \\ \mathrm{C}(46) & 35(1) & 35(1) & 33(1) & 2(1) & 11(1) & 0(1) \\ \mathrm{C}(47) & 36(1) & 36(2) & 42(1) & 2(1) & 14(1) & 0(1) \\ \mathrm{C}(48) & 40(1) & 31(1) & 47(1) & 3(1) & 25(1) & 0(1) \\ \mathrm{C}(49) & 42(1) & 24(1) & 35(1) & 0(1) & 23(1) & 0(1) \\ \mathrm{C}(50) & 49(1) & 24(1) & 33(1) & -2(1) & 26(1) & 0(1) \\ \mathrm{C}(51) & 66(2) & 41(2) & 36(1) & -11(1) & 17(1) & 5(1) \\ \mathrm{C}(52) & 52(2) & 31(2) & 47(2) & 5(1) & 10(1) & 7(1) \\ \mathrm{C}(53) & 53(2) & 38(2) & 45(2) & -2(1) & -7(1) & 9(1) \\ \mathrm{C}(54) & 55(2) & 33(2) & 37(1) & -8(1) & -6(1) & 3(1) \\ \mathrm{C}(55) & 37(3) & 100(7) & 91(6) & 21(5) & 24(3) & 10(3) \\ & & & & 48 & & \\ & & & & & & \\ & & & \end{array}$




\begin{tabular}{lllllll}
$\mathrm{C}(55 \mathrm{~B})$ & $56(6)$ & $58(8)$ & $105(12)$ & $-6(7)$ & $29(7)$ & $16(5)$ \\
$\mathrm{C}(56)$ & $35(1)$ & $77(2)$ & $37(1)$ & $12(2)$ & $5(1)$ & $6(1)$ \\
$\mathrm{C}(57)$ & $64(2)$ & $30(1)$ & $48(2)$ & $3(1)$ & $32(1)$ & $-8(1)$ \\
$\mathrm{C}(58)$ & $50(2)$ & $26(1)$ & $40(1)$ & $4(1)$ & $22(1)$ & $-2(1)$ \\
\hline
\end{tabular}

Table S7. Hydrogen coordinates $\left(\times 10^{4}\right)$ and isotropic displacement parameters $\left(\AA^{2} \times 10^{3}\right)$ for $\mathbf{A}$.

\begin{tabular}{lrrrl}
\hline & $x$ & $y$ & $z$ & $U(e q)$ \\
\hline$H(2)$ & 11073 & 3022 & 5931 & 49 \\
$H(1 A)$ & 7679 & 3995 & 7944 & 52 \\
$H(1 B)$ & 8046 & 2204 & 7946 & 52 \\
$H(2 A)$ & 9484 & -1291 & 5466 & 40 \\
$H(2 B)$ & 9941 & 4042 & 6528 & 35 \\
$H(3)$ & 11563 & 3485 & 7177 & 39 \\
$H(4 A)$ & 11430 & 1230 & 6695 & 36 \\
$H(5)$ & 12474 & 3685 & 6384 & 41 \\
$H(6 A)$ & 12313 & 1435 & 5870 & 43 \\
$H(6 B)$ & 13267 & 1464 & 6176 & 43 \\
$H(7)$ & 12064 & -828 & 6404 & 44 \\
$H(8 A)$ & 13094 & -1733 & 5777 & 46 \\
$H(8 B)$ & 12437 & -2972 & 5937 & 46 \\
$H(12 B)$ & 9984 & 68 & 4446 & 44 \\
$H(12 A)$ & 9988 & -216 & 4415 & 44 \\
$H(17)$ & 8775 & 363 & 5724 & 41 \\
$H(18)$ & 7014 & 359 & 5757 & 45 \\
$H(19)$ & 7444 & 944 & 6500 & 43 \\
$H(20)$ & 9155 & 950 & 6385 & 35 \\
$H(21)$ & 8697 & 3441 & 6743 & 35 \\
$H(22 A)$ & 10923 & 1974 & 7618 & 60 \\
$H(22 B)$ & 10384 & 410 & 7355 & 60 \\
$H(22 C)$ & 10038 & 1447 & 7731 & 60 \\
$H(23 A)$ & 11318 & 5741 & 6413 & 58
\end{tabular}




\begin{tabular}{|c|c|c|c|c|}
\hline $\mathrm{H}(23 \mathrm{~B})$ & 12117 & 5691 & 6846 & 58 \\
\hline$H(23 C)$ & 11215 & 6377 & 6902 & 58 \\
\hline$H(24 A)$ & 14081 & 2405 & 6869 & 81 \\
\hline $\mathrm{H}(24 \mathrm{~B})$ & 14016 & 3240 & 7344 & 81 \\
\hline $\mathrm{H}(24 \mathrm{C})$ & 13697 & 4281 & 6877 & 81 \\
\hline$H(25 A)$ & 13886 & -1045 & 6577 & 73 \\
\hline $\mathrm{H}(25 \mathrm{~B})$ & 13303 & -2506 & 6714 & 73 \\
\hline$H(25 C)$ & 13362 & -678 & 6955 & 73 \\
\hline $\mathrm{H}(26 \mathrm{~A})$ & 13735 & -180 & 4692 & 148 \\
\hline $\mathrm{H}(26 \mathrm{~B})$ & 12926 & -1308 & 4451 & 148 \\
\hline$H(26 C)$ & 12847 & 723 & 4462 & 148 \\
\hline$H(26 D)$ & 13732 & -448 & 4710 & 148 \\
\hline $\mathrm{H}(26 \mathrm{E})$ & 12858 & -886 & 4348 & 148 \\
\hline$H(26 F)$ & 12985 & 940 & 4591 & 148 \\
\hline $\mathrm{H}(27 \mathrm{~A})$ & 6942 & 223 & 4649 & 69 \\
\hline $\mathrm{H}(27 \mathrm{~B})$ & 6705 & -732 & 5071 & 69 \\
\hline $\mathrm{H}(27 \mathrm{C})$ & 7094 & -1786 & 4714 & 69 \\
\hline $\mathrm{H}(28 \mathrm{~A})$ & 8625 & -2363 & 6388 & 67 \\
\hline $\mathrm{H}(28 \mathrm{~B})$ & 9313 & -3062 & 6825 & 67 \\
\hline $\mathrm{H}(28 \mathrm{C})$ & 9608 & -1844 & 6465 & 67 \\
\hline $\mathrm{H}(12)$ & 14404 & -1579 & 9000 & 54 \\
\hline$H(3 A)$ & 18579 & -1044 & 7501 & 53 \\
\hline $\mathrm{H}(3 \mathrm{~B})$ & 18181 & -2810 & 7485 & 53 \\
\hline $\mathrm{H}(4)$ & 15761 & -5898 & 9666 & 41 \\
\hline$H(31)$ & 15878 & -586 & 8594 & 41 \\
\hline $\mathrm{H}(32)$ & 14485 & -1105 & 7793 & 44 \\
\hline $\mathrm{H}(33)$ & 14371 & -3340 & 8253 & 40 \\
\hline $\mathrm{H}(34)$ & 13195 & -838 & 8426 & 41 \\
\hline$H(35 A)$ & 12269 & -3106 & 8486 & 42 \\
\hline $\mathrm{H}(35 \mathrm{~B})$ & 13104 & -3100 & 8896 & 42 \\
\hline $\mathrm{H}(36)$ & 13663 & -5330 & 8470 & 42 \\
\hline $\mathrm{H}(37 \mathrm{~A})$ & 12228 & -6291 & 8824 & 45 \\
\hline $\mathrm{H}(37 \mathrm{~B})$ & 12982 & -7541 & 8775 & 45 \\
\hline $\mathrm{H}(41 \mathrm{~A})$ & 14702 & -4625 & 10509 & 41 \\
\hline $\mathrm{H}(41)$ & 14692 & -4844 & 10530 & 41 \\
\hline $\mathrm{H}(46)$ & 16613 & -4361 & 9519 & 41 \\
\hline
\end{tabular}




\begin{tabular}{|c|c|c|c|c|}
\hline $\mathrm{H}(47)$ & 18442 & -4242 & 9747 & 44 \\
\hline $\mathrm{H}(48)$ & 18370 & -3857 & 9008 & 44 \\
\hline $\mathrm{H}(49)$ & 16547 & -3929 & 8856 & 37 \\
\hline$H(50)$ & 17219 & -1455 & 8582 & 39 \\
\hline$H(51 A)$ & 15263 & -2990 & 7530 & 71 \\
\hline $\mathrm{H}(51 \mathrm{~B})$ & 15712 & -4512 & 7853 & 71 \\
\hline$H(51 C)$ & 16209 & -3576 & 7521 & 71 \\
\hline$H(52 A)$ & 13803 & 1146 & 8050 & 66 \\
\hline $\mathrm{H}(52 \mathrm{~B})$ & 14757 & 1771 & 8076 & 66 \\
\hline $\mathrm{H}(52 \mathrm{C})$ & 14489 & 1192 & 8531 & 66 \\
\hline$H(53 A)$ & 12005 & -1138 & 7347 & 73 \\
\hline$H(53 B)$ & 12175 & -96 & 7818 & 73 \\
\hline$H(53 C)$ & 11741 & -1941 & 7783 & 73 \\
\hline$H(54 A)$ & 12579 & -6975 & 7961 & 67 \\
\hline$H(54 B)$ & 12678 & -5121 & 7758 & 67 \\
\hline$H(54 C)$ & 11899 & -5516 & 7985 & 67 \\
\hline$H(55 A)$ & 11005 & -4799 & 9683 & 112 \\
\hline$H(55 B)$ & 11783 & -3837 & 10020 & 112 \\
\hline $\mathrm{H}(55 \mathrm{C})$ & 11688 & -5860 & 10053 & 112 \\
\hline$H(55 D)$ & 11432 & -3781 & 9301 & 107 \\
\hline$H(55 E)$ & 11885 & -3431 & 9827 & 107 \\
\hline$H(55 F)$ & 11027 & -4532 & 9696 & 107 \\
\hline$H(56 A)$ & 17933 & -4167 & 10762 & 76 \\
\hline$H(56 B)$ & 18424 & -5124 & 10432 & 76 \\
\hline $\mathrm{H}(56 \mathrm{C})$ & 17870 & -6195 & 10709 & 76 \\
\hline $\mathrm{H}(57 \mathrm{~A})$ & 16759 & -8002 & 8491 & 66 \\
\hline$H(57 B)$ & 16204 & -6838 & 8746 & 66 \\
\hline$H(57 C)$ & 17194 & -7153 & 8974 & 66 \\
\hline
\end{tabular}

Table S8. Torsion angles $\left[^{\circ}\right]$ for $\mathbf{A}$.

\begin{tabular}{lr}
\hline$C(2)-O(1)-C(1)-C(22)$ & $115.1(2)$ \\
$C(2)-O(1)-C(1)-C(21)$ & $-106.5(2)$ \\
$C(1)-O(1)-C(2)-C(3)$ & $-118.4(2)$
\end{tabular}




\begin{tabular}{|c|c|}
\hline$C(22)-C(1)-C(2)-O(1)$ & $-101.8(3)$ \\
\hline$C(21)-C(1)-C(2)-O(1)$ & $101.9(2)$ \\
\hline$O(1)-C(1)-C(2)-C(3)$ & $101.4(3)$ \\
\hline$C(22)-C(1)-C(2)-C(3)$ & $-0.4(4)$ \\
\hline$C(21)-C(1)-C(2)-C(3)$ & $-156.7(2)$ \\
\hline$O(1)-C(2)-C(3)-C(23)$ & $-75.3(3)$ \\
\hline$C(1)-C(2)-C(3)-C(23)$ & $-145.2(2)$ \\
\hline $\mathrm{O}(1)-\mathrm{C}(2)-\mathrm{C}(3)-\mathrm{C}(4)$ & $158.4(2)$ \\
\hline$C(1)-C(2)-C(3)-C(4)$ & $88.5(3)$ \\
\hline $\mathrm{C}(2)-\mathrm{C}(3)-\mathrm{C}(4)-\mathrm{O}(2)$ & $46.3(3)$ \\
\hline$C(23)-C(3)-C(4)-O(2)$ & $-76.1(3)$ \\
\hline$C(2)-C(3)-C(4)-C(5)$ & $171.1(2)$ \\
\hline$C(23)-C(3)-C(4)-C(5)$ & $48.8(3)$ \\
\hline$C(24)-O(3)-C(5)-C(4)$ & $-159.6(2)$ \\
\hline $\mathrm{C}(24)-\mathrm{O}(3)-\mathrm{C}(5)-\mathrm{C}(6)$ & $74.0(3)$ \\
\hline $\mathrm{O}(2)-\mathrm{C}(4)-\mathrm{C}(5)-\mathrm{O}(3)$ & $-177.4(2)$ \\
\hline$C(3)-C(4)-C(5)-O(3)$ & $57.0(3)$ \\
\hline$O(2)-C(4)-C(5)-C(6)$ & $-51.3(3)$ \\
\hline$C(3)-C(4)-C(5)-C(6)$ & $-176.9(2)$ \\
\hline $\mathrm{O}(3)-\mathrm{C}(5)-\mathrm{C}(6)-\mathrm{C}(7)$ & $62.4(3)$ \\
\hline$C(4)-C(5)-C(6)-C(7)$ & $-59.1(3)$ \\
\hline$C(5)-C(6)-C(7)-C(25)$ & $-80.5(3)$ \\
\hline$C(5)-C(6)-C(7)-C(8)$ & $158.1(2)$ \\
\hline$C(25)-C(7)-C(8)-C(9)$ & $-176.5(2)$ \\
\hline$C(6)-C(7)-C(8)-C(9)$ & $-52.9(3)$ \\
\hline$C(7)-C(8)-C(9)-C(14)$ & $108.8(9)$ \\
\hline$C(7)-C(8)-C(9)-C(14 B)$ & $126.8(9)$ \\
\hline$C(7)-C(8)-C(9)-C(10)$ & $-63.2(3)$ \\
\hline$C(14)-C(9)-C(10)-O(4)$ & $-173.5(8)$ \\
\hline$C(14 B)-C(9)-C(10)-O(4)$ & $169.4(8)$ \\
\hline$C(8)-C(9)-C(10)-O(4)$ & $-1.3(4)$ \\
\hline$C(14)-C(9)-C(10)-C(11)$ & $6.8(9)$ \\
\hline$C(14 B)-C(9)-C(10)-C(11)$ & $-10.3(8)$ \\
\hline$C(8)-C(9)-C(10)-C(11)$ & $179.0(2)$ \\
\hline$C(15)-N(2)-C(11)-C(12)$ & $0.4(5)$ \\
\hline$C(15)-N(2)-C(11)-C(10)$ & $-179.9(2)$ \\
\hline
\end{tabular}




\begin{tabular}{|c|c|}
\hline $\mathrm{O}(4)-\mathrm{C}(10)-\mathrm{C}(11)-\mathrm{C}(12)$ & $-176.5(3)$ \\
\hline$C(9)-C(10)-C(11)-C(12)$ & $3.3(4)$ \\
\hline $\mathrm{O}(4)-\mathrm{C}(10)-\mathrm{C}(11)-\mathrm{N}(2)$ & $3.8(3)$ \\
\hline$C(9)-C(10)-C(11)-N(2)$ & $-176.4(2)$ \\
\hline$N(2)-C(11)-C(12)-C(13 B)$ & $-174.3(9)$ \\
\hline$C(10)-C(11)-C(12)-C(13 B)$ & $6.1(9)$ \\
\hline$N(2)-C(11)-C(12)-C(13)$ & $169.0(9)$ \\
\hline$C(10)-C(11)-C(12)-C(13)$ & $-10.7(9)$ \\
\hline$C(11)-C(12)-C(13)-O(6)$ & $-166.8(11)$ \\
\hline$C(11)-C(12)-C(13)-C(14)$ & $9.1(17)$ \\
\hline$C(11)-C(12)-C(13 B)-O(6 B)$ & $172.0(11)$ \\
\hline$C(11)-C(12)-C(13 B)-C(14 B)$ & $-8.0(16)$ \\
\hline $\mathrm{C}(26)-\mathrm{O}(5)-\mathrm{C}(14)-\mathrm{C}(9)$ & $155.1(13)$ \\
\hline $\mathrm{C}(26)-\mathrm{O}(5)-\mathrm{C}(14)-\mathrm{C}(13)$ & $-19.4(19)$ \\
\hline $\mathrm{C}(10)-\mathrm{C}(9)-\mathrm{C}(14)-\mathrm{O}(5)$ & $177.0(8)$ \\
\hline $\mathrm{C}(8)-\mathrm{C}(9)-\mathrm{C}(14)-\mathrm{O}(5)$ & $5.1(15)$ \\
\hline$C(10)-C(9)-C(14)-C(13)$ & $-8.3(16)$ \\
\hline$C(8)-C(9)-C(14)-C(13)$ & $179.9(9)$ \\
\hline $\mathrm{O}(6)-\mathrm{C}(13)-\mathrm{C}(14)-\mathrm{O}(5)$ & $-9(2)$ \\
\hline$C(12)-C(13)-C(14)-O(5)$ & $175.1(12)$ \\
\hline $\mathrm{O}(6)-\mathrm{C}(13)-\mathrm{C}(14)-\mathrm{C}(9)$ & $176.7(12)$ \\
\hline$C(12)-C(13)-C(14)-C(9)$ & $1(2)$ \\
\hline $\mathrm{C}(26)-\mathrm{O}(5 \mathrm{~B})-\mathrm{C}(14 \mathrm{~B})-\mathrm{C}(9)$ & $-164.4(15)$ \\
\hline $\mathrm{C}(26)-\mathrm{O}(5 \mathrm{~B})-\mathrm{C}(14 \mathrm{~B})-\mathrm{C}(13 \mathrm{~B})$ & $9(2)$ \\
\hline $\mathrm{C}(10)-\mathrm{C}(9)-\mathrm{C}(14 \mathrm{~B})-\mathrm{O}(5 \mathrm{~B})$ & $-178.1(9)$ \\
\hline $\mathrm{C}(8)-\mathrm{C}(9)-\mathrm{C}(14 \mathrm{~B})-\mathrm{O}(5 \mathrm{~B})$ & $-8.1(15)$ \\
\hline$C(10)-C(9)-C(14 B)-C(13 B)$ & $8.4(16)$ \\
\hline $\mathrm{C}(8)-\mathrm{C}(9)-\mathrm{C}(14 \mathrm{~B})-\mathrm{C}(13 \mathrm{~B})$ & $178.4(10)$ \\
\hline $\mathrm{O}(6 \mathrm{~B})-\mathrm{C}(13 \mathrm{~B})-\mathrm{C}(14 \mathrm{~B})-\mathrm{O}(5 \mathrm{~B})$ & $7(2)$ \\
\hline$C(12)-C(13 B)-C(14 B)-O(5 B)$ & $-173.2(11)$ \\
\hline $\mathrm{O}(6 \mathrm{~B})-\mathrm{C}(13 \mathrm{~B})-\mathrm{C}(14 \mathrm{~B})-\mathrm{C}(9)$ & $-179.6(12)$ \\
\hline$C(12)-C(13 B)-C(14 B)-C(9)$ & $0.5(19)$ \\
\hline $\mathrm{C}(11)-\mathrm{N}(2)-\mathrm{C}(15)-\mathrm{O}(7)$ & $14.5(4)$ \\
\hline$C(11)-N(2)-C(15)-C(16)$ & $-164.7(2)$ \\
\hline $\mathrm{O}(7)-\mathrm{C}(15)-\mathrm{C}(16)-\mathrm{C}(17)$ & $-151.9(3)$ \\
\hline$N(2)-C(15)-C(16)-C(17)$ & $27.3(4)$ \\
\hline
\end{tabular}




\begin{tabular}{|c|c|}
\hline $\mathrm{O}(7)-\mathrm{C}(15)-\mathrm{C}(16)-\mathrm{C}(27)$ & $25.1(4)$ \\
\hline$N(2)-C(15)-C(16)-C(27)$ & $-155.6(3)$ \\
\hline$C(15)-C(16)-C(17)-C(18)$ & $-179.8(3)$ \\
\hline$C(27)-C(16)-C(17)-C(18)$ & $3.5(5)$ \\
\hline$C(16)-C(17)-C(18)-C(19)$ & $172.1(3)$ \\
\hline$C(17)-C(18)-C(19)-C(20)$ & $-1.4(5)$ \\
\hline $\mathrm{C}(28)-\mathrm{O}(8)-\mathrm{C}(20)-\mathrm{C}(19)$ & $75.8(3)$ \\
\hline$C(28)-O(8)-C(20)-C(21)$ & $-160.8(2)$ \\
\hline $\mathrm{C}(18)-\mathrm{C}(19)-\mathrm{C}(20)-\mathrm{O}(8)$ & $-104.3(3)$ \\
\hline$C(18)-C(19)-C(20)-C(21)$ & $134.7(3)$ \\
\hline $\mathrm{C}(29)-\mathrm{O}(9)-\mathrm{C}(21)-\mathrm{C}(1)$ & $93.9(2)$ \\
\hline$C(29)-O(9)-C(21)-C(20)$ & $-142.2(2)$ \\
\hline $\mathrm{O}(1)-\mathrm{C}(1)-\mathrm{C}(21)-\mathrm{O}(9)$ & $-88.8(2)$ \\
\hline$C(2)-C(1)-C(21)-O(9)$ & $-154.2(2)$ \\
\hline$C(22)-C(1)-C(21)-O(9)$ & $48.2(3)$ \\
\hline$O(1)-C(1)-C(21)-C(20)$ & $150.47(19)$ \\
\hline$C(2)-C(1)-C(21)-C(20)$ & $85.1(3)$ \\
\hline$C(22)-C(1)-C(21)-C(20)$ & $-72.5(3)$ \\
\hline $\mathrm{O}(8)-\mathrm{C}(20)-\mathrm{C}(21)-\mathrm{O}(9)$ & $-53.1(2)$ \\
\hline$C(19)-C(20)-C(21)-O(9)$ & $68.9(2)$ \\
\hline $\mathrm{O}(8)-\mathrm{C}(20)-\mathrm{C}(21)-\mathrm{C}(1)$ & $68.8(2)$ \\
\hline$C(19)-C(20)-C(21)-C(1)$ & $-169.1(2)$ \\
\hline $\mathrm{C}(21)-\mathrm{O}(9)-\mathrm{C}(29)-\mathrm{O}(10)$ & $15.1(4)$ \\
\hline $\mathrm{C}(21)-\mathrm{O}(9)-\mathrm{C}(29)-\mathrm{N}(1)$ & $-165.9(2)$ \\
\hline$C(31)-O(11)-C(30)-C(50)$ & $-107.5(2)$ \\
\hline $\mathrm{C}(31)-\mathrm{O}(11)-\mathrm{C}(30)-\mathrm{C}(51)$ & $114.8(3)$ \\
\hline $\mathrm{C}(30)-\mathrm{O}(11)-\mathrm{C}(31)-\mathrm{C}(32)$ & $-118.0(3)$ \\
\hline$C(50)-C(30)-C(31)-O(11)$ & $100.4(2)$ \\
\hline $\mathrm{C}(51)-\mathrm{C}(30)-\mathrm{C}(31)-\mathrm{O}(11)$ & $-101.0(3)$ \\
\hline$O(11)-C(30)-C(31)-C(32)$ & 102.3(3) \\
\hline$C(50)-C(30)-C(31)-C(32)$ & $-157.3(2)$ \\
\hline$C(51)-C(30)-C(31)-C(32)$ & $1.3(4)$ \\
\hline $\mathrm{O}(11)-\mathrm{C}(31)-\mathrm{C}(32)-\mathrm{C}(52)$ & $-81.1(3)$ \\
\hline$C(30)-C(31)-C(32)-C(52)$ & $-151.7(2)$ \\
\hline $\mathrm{O}(11)-\mathrm{C}(31)-\mathrm{C}(32)-\mathrm{C}(33)$ & 153.3(2) \\
\hline$C(30)-C(31)-C(32)-C(33)$ & $82.7(3)$ \\
\hline
\end{tabular}


$\mathrm{C}(31)-\mathrm{C}(32)-\mathrm{C}(33)-\mathrm{O}(12)$

$\mathrm{C}(52)-\mathrm{C}(32)-\mathrm{C}(33)-\mathrm{O}(12)$

$\mathrm{C}(31)-\mathrm{C}(32)-\mathrm{C}(33)-\mathrm{C}(34)$

$\mathrm{C}(52)-\mathrm{C}(32)-\mathrm{C}(33)-\mathrm{C}(34)$

$\mathrm{C}(53)-\mathrm{O}(13)-\mathrm{C}(34)-\mathrm{C}(35)$

$\mathrm{C}(53)-\mathrm{O}(13)-\mathrm{C}(34)-\mathrm{C}(33)$

$\mathrm{O}(12)-\mathrm{C}(33)-\mathrm{C}(34)-\mathrm{O}(13)$

$\mathrm{C}(32)-\mathrm{C}(33)-\mathrm{C}(34)-\mathrm{O}(13)$

$\mathrm{O}(12)-\mathrm{C}(33)-\mathrm{C}(34)-\mathrm{C}(35)$

$\mathrm{C}(32)-\mathrm{C}(33)-\mathrm{C}(34)-\mathrm{C}(35)$

$\mathrm{O}(13)-\mathrm{C}(34)-\mathrm{C}(35)-\mathrm{C}(36)$

$\mathrm{C}(33)-\mathrm{C}(34)-\mathrm{C}(35)-\mathrm{C}(36)$

$\mathrm{C}(34)-\mathrm{C}(35)-\mathrm{C}(36)-\mathrm{C}(37)$

$\mathrm{C}(34)-\mathrm{C}(35)-\mathrm{C}(36)-\mathrm{C}(54)$

$\mathrm{C}(35)-\mathrm{C}(36)-\mathrm{C}(37)-\mathrm{C}(38)$

$\mathrm{C}(54)-\mathrm{C}(36)-\mathrm{C}(37)-\mathrm{C}(38)$

$\mathrm{C}(36)-\mathrm{C}(37)-\mathrm{C}(38)-\mathrm{C}(43)$

$\mathrm{C}(36)-\mathrm{C}(37)-\mathrm{C}(38)-\mathrm{C}(43 \mathrm{~B})$

$\mathrm{C}(36)-\mathrm{C}(37)-\mathrm{C}(38)-\mathrm{C}(39)$

$\mathrm{C}(43)-\mathrm{C}(38)-\mathrm{C}(39)-\mathrm{O}(16)$

C(43B)-C(38)-C(39)-O(16)

$\mathrm{C}(37)-\mathrm{C}(38)-\mathrm{C}(39)-\mathrm{O}(16)$

$\mathrm{C}(43)-\mathrm{C}(38)-\mathrm{C}(39)-\mathrm{C}(40)$

$\mathrm{C}(43 \mathrm{~B})-\mathrm{C}(38)-\mathrm{C}(39)-\mathrm{C}(40)$

$\mathrm{C}(37)-\mathrm{C}(38)-\mathrm{C}(39)-\mathrm{C}(40)$

$\mathrm{C}(44)-\mathrm{N}(4)-\mathrm{C}(40)-\mathrm{C}(41)$

C(44)-N(4)-C(40)-C(39)

$\mathrm{O}(16)-\mathrm{C}(39)-\mathrm{C}(40)-\mathrm{C}(41)$

$\mathrm{C}(38)-\mathrm{C}(39)-\mathrm{C}(40)-\mathrm{C}(41)$

$\mathrm{O}(16)-\mathrm{C}(39)-\mathrm{C}(40)-\mathrm{N}(4)$

C(38)-C(39)-C(40)-N(4)

$\mathrm{N}(4)-\mathrm{C}(40)-\mathrm{C}(41)-\mathrm{C}(42)$

$\mathrm{C}(39)-\mathrm{C}(40)-\mathrm{C}(41)-\mathrm{C}(42)$

$\mathrm{N}(4)-\mathrm{C}(40)-\mathrm{C}(41)-\mathrm{C}(42 \mathrm{~B})$

$\mathrm{C}(39)-\mathrm{C}(40)-\mathrm{C}(41)-\mathrm{C}(42 \mathrm{~B})$

$\mathrm{C}(40)-\mathrm{C}(41)-\mathrm{C}(42)-\mathrm{O}(15)$
46.6(3)

$-75.2(3)$

170.9(2)

49.1(3)

75.3(3)

$-159.2(2)$

$-179.7(2)$

$54.9(3)$

$-53.8(3)$

$-179.2(2)$

65.3(3)

$-55.1(3)$

162.7(2)

$-75.7(3)$

$-55.8(3)$

$-178.8(2)$

$110.8(8)$

128.5(11)

$-61.6(3)$

$-175.7(8)$

167.4(11)

$-2.9(4)$

$3.8(8)$

$-13.1(11)$

176.6(2)

$-0.5(5)$

178.6(2)

$-176.3(2)$

4.2(4)

4.5(3)

$-175.0(2)$

172.3(10)

$-6.8(10)$

$-174.9(12)$

6.0(13)

$-176.1(12)$ 
$\mathrm{C}(40)-\mathrm{C}(41)-\mathrm{C}(42)-\mathrm{C}(43)$

$\mathrm{C}(40)-\mathrm{C}(41)-\mathrm{C}(42 \mathrm{~B})-\mathrm{O}(14 \mathrm{~B})$

$\mathrm{C}(40)-\mathrm{C}(41)-\mathrm{C}(42 \mathrm{~B})-\mathrm{C}(43 \mathrm{~B})$

$\mathrm{C}(55)-\mathrm{O}(14)-\mathrm{C}(43)-\mathrm{C}(38)$

$\mathrm{C}(55)-\mathrm{O}(14)-\mathrm{C}(43)-\mathrm{C}(42)$

$\mathrm{C}(39)-\mathrm{C}(38)-\mathrm{C}(43)-\mathrm{O}(14)$

$\mathrm{C}(37)-\mathrm{C}(38)-\mathrm{C}(43)-\mathrm{O}(14)$

$\mathrm{C}(39)-\mathrm{C}(38)-\mathrm{C}(43)-\mathrm{C}(42)$

$\mathrm{C}(37)-\mathrm{C}(38)-\mathrm{C}(43)-\mathrm{C}(42)$

$\mathrm{O}(15)-\mathrm{C}(42)-\mathrm{C}(43)-\mathrm{O}(14)$

$\mathrm{C}(41)-\mathrm{C}(42)-\mathrm{C}(43)-\mathrm{O}(14)$

$\mathrm{O}(15)-\mathrm{C}(42)-\mathrm{C}(43)-\mathrm{C}(38)$

$\mathrm{C}(41)-\mathrm{C}(42)-\mathrm{C}(43)-\mathrm{C}(38)$

$\mathrm{C}(55 \mathrm{~B})-\mathrm{O}(13 \mathrm{~B})-\mathrm{C}(43 \mathrm{~B})-\mathrm{C}(38)$

C(55B)-O(13B)-C(43B)-C(42B)

$\mathrm{C}(39)-\mathrm{C}(38)-\mathrm{C}(43 \mathrm{~B})-\mathrm{O}(13 \mathrm{~B})$

$\mathrm{C}(37)-\mathrm{C}(38)-\mathrm{C}(43 \mathrm{~B})-\mathrm{O}(13 \mathrm{~B})$

$\mathrm{C}(39)-\mathrm{C}(38)-\mathrm{C}(43 \mathrm{~B})-\mathrm{C}(42 \mathrm{~B})$

$\mathrm{C}(37)-\mathrm{C}(38)-\mathrm{C}(43 \mathrm{~B})-\mathrm{C}(42 \mathrm{~B})$

$\mathrm{O}(14 \mathrm{~B})-\mathrm{C}(42 \mathrm{~B})-\mathrm{C}(43 \mathrm{~B})-\mathrm{O}(13 \mathrm{~B})$

$\mathrm{C}(41)-\mathrm{C}(42 \mathrm{~B})-\mathrm{C}(43 \mathrm{~B})-\mathrm{O}(13 \mathrm{~B})$

$\mathrm{O}(14 \mathrm{~B})-\mathrm{C}(42 \mathrm{~B})-\mathrm{C}(43 \mathrm{~B})-\mathrm{C}(38)$

$\mathrm{C}(41)-\mathrm{C}(42 \mathrm{~B})-\mathrm{C}(43 \mathrm{~B})-\mathrm{C}(38)$

$\mathrm{C}(40)-\mathrm{N}(4)-\mathrm{C}(44)-\mathrm{O}(17)$

$\mathrm{C}(40)-\mathrm{N}(4)-\mathrm{C}(44)-\mathrm{C}(45)$

$\mathrm{O}(17)-\mathrm{C}(44)-\mathrm{C}(45)-\mathrm{C}(46)$

$\mathrm{N}(4)-\mathrm{C}(44)-\mathrm{C}(45)-\mathrm{C}(46)$

$\mathrm{O}(17)-\mathrm{C}(44)-\mathrm{C}(45)-\mathrm{C}(56)$

$\mathrm{N}(4)-\mathrm{C}(44)-\mathrm{C}(45)-\mathrm{C}(56)$

$\mathrm{C}(44)-\mathrm{C}(45)-\mathrm{C}(46)-\mathrm{C}(47)$

$\mathrm{C}(56)-\mathrm{C}(45)-\mathrm{C}(46)-\mathrm{C}(47)$

$\mathrm{C}(45)-\mathrm{C}(46)-\mathrm{C}(47)-\mathrm{C}(48)$

$\mathrm{C}(46)-\mathrm{C}(47)-\mathrm{C}(48)-\mathrm{C}(49)$

$\mathrm{C}(57)-\mathrm{O}(18)-\mathrm{C}(49)-\mathrm{C}(48)$

$\mathrm{C}(57)-\mathrm{O}(18)-\mathrm{C}(49)-\mathrm{C}(50)$

$\mathrm{C}(47)-\mathrm{C}(48)-\mathrm{C}(49)-\mathrm{O}(18)$
2.2(19)

168.7(16)

$-7(2)$

162.7(15)

$-13(2)$

176.3(8)

$3.9(14)$

$-8.4(16)$

179.2(10)

$-1(2)$

$-179.1(12)$

$-175.8(13)$

6(2)

$-104(2)$

$73(2)$

$-171.1(13)$

$-1(2)$

12(2)

$-178.5(15)$

5(3)

$-179.3(16)$

$-177.9(19)$

$-2(3)$

14.6(4)

$-165.3(3)$

$-155.8(3)$

24.1(4)

21.3(4)

$-158.8(3)$

$-179.2(3)$

3.9(5)

172.0(3)

$-0.5(5)$

72.8(3)

$-164.3(2)$

$-106.7(3)$ 
$\mathrm{C}(47)-\mathrm{C}(48)-\mathrm{C}(49)-\mathrm{C}(50)$

$\mathrm{C}(58)-\mathrm{O}(19)-\mathrm{C}(50)-\mathrm{C}(30)$

$\mathrm{C}(58)-\mathrm{O}(19)-\mathrm{C}(50)-\mathrm{C}(49)$

$\mathrm{O}(11)-\mathrm{C}(30)-\mathrm{C}(50)-\mathrm{O}(19)$

$\mathrm{C}(31)-\mathrm{C}(30)-\mathrm{C}(50)-\mathrm{O}(19)$

$\mathrm{C}(51)-\mathrm{C}(30)-\mathrm{C}(50)-\mathrm{O}(19)$

$\mathrm{O}(11)-\mathrm{C}(30)-\mathrm{C}(50)-\mathrm{C}(49)$

$\mathrm{C}(31)-\mathrm{C}(30)-\mathrm{C}(50)-\mathrm{C}(49)$

$\mathrm{C}(51)-\mathrm{C}(30)-\mathrm{C}(50)-\mathrm{C}(49)$

$\mathrm{O}(18)-\mathrm{C}(49)-\mathrm{C}(50)-\mathrm{O}(19)$

$\mathrm{C}(48)-\mathrm{C}(49)-\mathrm{C}(50)-\mathrm{O}(19)$

$\mathrm{O}(18)-\mathrm{C}(49)-\mathrm{C}(50)-\mathrm{C}(30)$

$\mathrm{C}(48)-\mathrm{C}(49)-\mathrm{C}(50)-\mathrm{C}(30)$

$\mathrm{C}(50)-\mathrm{O}(19)-\mathrm{C}(58)-\mathrm{O}(20)$

$\mathrm{C}(50)-\mathrm{O}(19)-\mathrm{C}(58)-\mathrm{N}(3)$
132.7(3)

88.0(3)

$-147.7(2)$

$-84.7(2)$

$-149.4(2)$

51.1(3)

155.14(19)

90.4(3)

$-69.1(3)$

$-48.7(3)$

$73.4(3)$

$72.6(2)$

$-165.3(2)$

26.2(4)

$-156.2(2)$

Symmetry transformations used to generate equivalent atoms: 
Refinement details for $\mathbf{C}$

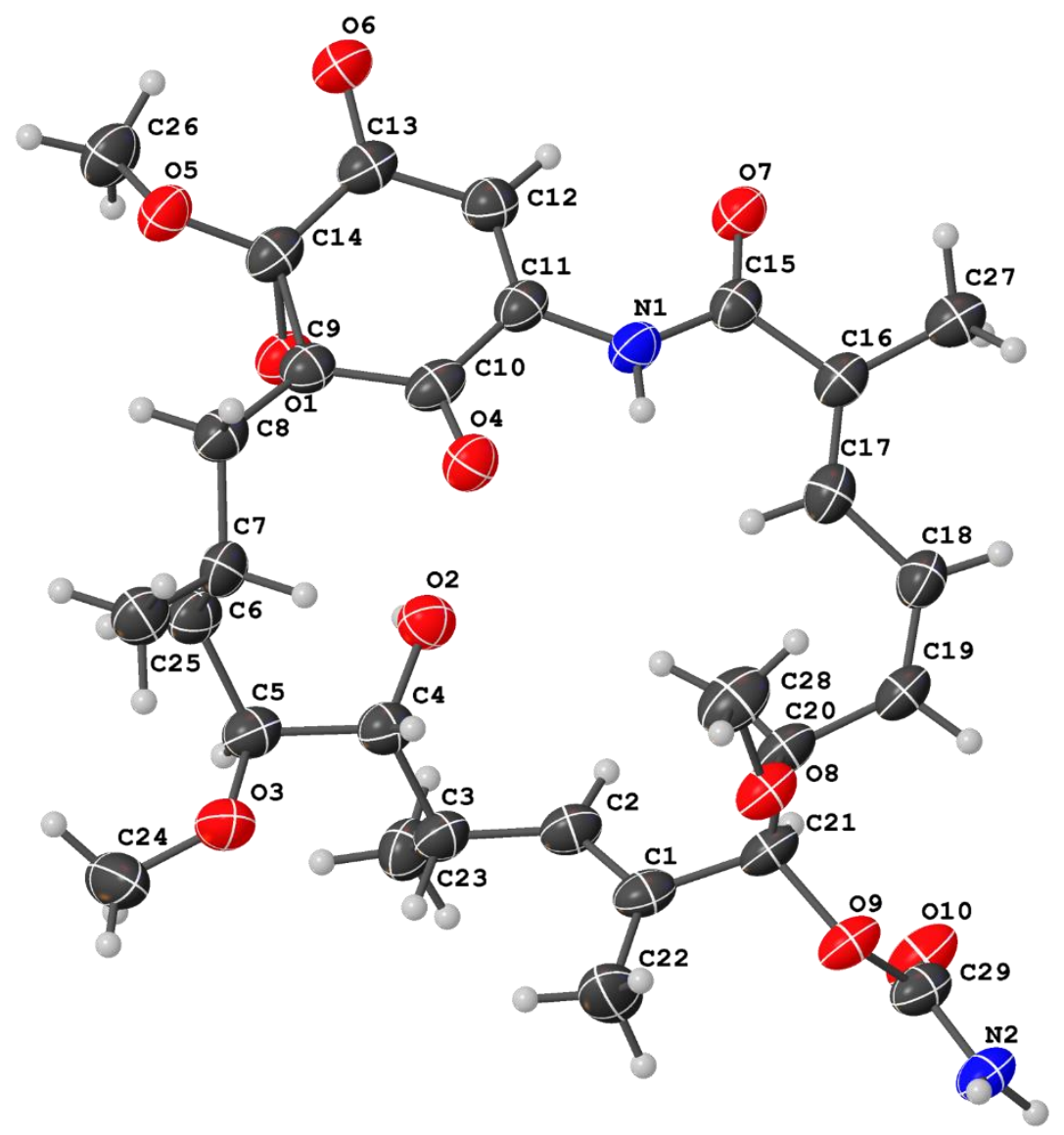

Figure S2. The complete numbering scheme of $\mathbf{C}$ with $50 \%$ thermal ellipsoid probability levels. The hydrogen atoms are shown as circles for clarity. 
Table S9. Atomic coordinates $\left(x 1^{4}\right)$ and equivalent isotropic displacement parameters $\left(\AA^{2} x\right.$ $10^{3}$ ) for $\mathbf{C} . \mathrm{U}(\mathrm{eq})$ is defined as one third of the trace of the orthogonalized Uij tensor.

\begin{tabular}{|c|c|c|c|c|}
\hline & $x$ & $y$ & $z$ & $\mathrm{U}(\mathrm{eq})$ \\
\hline $\mathrm{O}(1)$ & 2600(3) & $5687(5)$ & $5232(2)$ & $43(1)$ \\
\hline $\mathrm{O}(2)$ & $4290(3)$ & $4232(5)$ & 7004(2) & $45(1)$ \\
\hline $\mathrm{O}(3)$ & 2787(3) & $4933(6)$ & $8708(2)$ & $57(1)$ \\
\hline $\mathrm{O}(4)$ & 4495(3) & $8288(5)$ & $6476(2)$ & $44(1)$ \\
\hline $\mathrm{O}(5)$ & $1306(3)$ & $7646(5)$ & $4465(2)$ & $45(1)$ \\
\hline $\mathrm{O}(6)$ & $2686(3)$ & $7459(6)$ & $3284(2)$ & $55(1)$ \\
\hline $\mathrm{O}(7)$ & 6524(3) & $6744(5)$ & $4256(2)$ & $43(1)$ \\
\hline $\mathrm{O}(8)$ & 7696(3) & $7336(5)$ & $8817(2)$ & $46(1)$ \\
\hline O(9) & $8648(3)$ & $4284(5)$ & $9449(2)$ & $44(1)$ \\
\hline $\mathrm{O}(10)$ & 9292(3) & $1643(5)$ & 9089(3) & $58(1)$ \\
\hline $\mathrm{N}(1)$ & 5794(3) & $7421(6)$ & 5419(3) & $40(1)$ \\
\hline$N(2)$ & $10084(4)$ & $3163(6)$ & $10255(3)$ & $46(1)$ \\
\hline$C(1)$ & 6699(5) & $3884(7)$ & 9016(3) & $42(1)$ \\
\hline$C(2)$ & $5918(4)$ & $3243(7)$ & $8419(3)$ & $43(1)$ \\
\hline$C(3)$ & $4746(4)$ & $3005(7)$ & $8442(3)$ & $43(1)$ \\
\hline$C(4)$ & $4090(4)$ & $4361(7)$ & $7848(3)$ & $42(1)$ \\
\hline$C(5)$ & $2875(4)$ & $4310(8)$ & 7886(3) & $44(1)$ \\
\hline$C(6)$ & 2181(4) & $5339(7)$ & $7177(3)$ & $43(1)$ \\
\hline$C(7)$ & $2404(4)$ & $7331(8)$ & 7154(3) & $43(1)$ \\
\hline$C(8)$ & $2175(4)$ & $8073(7)$ & $6248(3)$ & $42(1)$ \\
\hline $\mathrm{C}(9)$ & $2847(4)$ & $7437(7)$ & $5612(3)$ & $40(1)$ \\
\hline$C(10)$ & $4045(4)$ & $7748(6)$ & $5786(3)$ & $40(1)$ \\
\hline$C(11)$ & $4694(4)$ & $7406(7)$ & $5107(3)$ & $39(1)$ \\
\hline$C(12)$ & $4247(4)$ & $7196(7)$ & 4291(3) & $44(1)$ \\
\hline$C(13)$ & $3071(4)$ & $7297(7)$ & 4033(3) & $44(1)$ \\
\hline$C(14)$ & $2367(4)$ & $7211(7)$ & $4697(3)$ & $41(1)$ \\
\hline$C(15)$ & $6643(4)$ & $7036(7)$ & $5018(3)$ & $38(1)$ \\
\hline$C(16)$ & 7722(4) & $6923(6)$ & $5565(3)$ & $38(1)$ \\
\hline$C(17)$ & $7791(4)$ & $6621(7)$ & $6390(4)$ & $44(1)$ \\
\hline$C(18)$ & $8761(4)$ & $6419(8)$ & 7009(3) & $44(1)$ \\
\hline$C(19)$ & $8764(4)$ & $6125(7)$ & $7835(3)$ & $44(1)$ \\
\hline
\end{tabular}




$\begin{array}{lllll}\mathrm{C}(20) & 7785(4) & 5905(7) & 8249(3) & 43(1) \\ \mathrm{C}(21) & 7771(4) & 4183(7) & 8728(3) & 42(1) \\ \mathrm{C}(22) & 6557(5) & 4424(11) & 9884(4) & 62(2) \\ \mathrm{C}(23) & 4421(5) & 1084(8) & 8212(4) & 49(1) \\ \mathrm{C}(24) & 1887(6) & 4314(12) & 9042(4) & 72(2) \\ \mathrm{C}(25) & 1736(5) & 8360(8) & 7703(4) & 49(1) \\ \mathrm{C}(26) & 701(5) & 6520(9) & 3828(4) & 53(2) \\ \mathrm{C}(27) & 8665(4) & 7019(7) & 5107(3) & 45(1) \\ \mathrm{C}(28) & 7277(6) & 8898(8) & 8392(4) & 60(2) \\ \mathrm{C}(29) & 9353(5) & 2907(7) & 9560(3) & 45(1)\end{array}$

Table S10. Bond lengths $[\AA]$ and angles $\left[{ }^{\circ}\right]$ for $\mathbf{C}$.

\begin{tabular}{ll}
\hline $\mathrm{O}(1)-\mathrm{C}(14)$ & $1.440(6)$ \\
$\mathrm{O}(1)-\mathrm{C}(9)$ & $1.471(6)$ \\
$\mathrm{O}(2)-\mathrm{C}(4)$ & $1.416(6)$ \\
$\mathrm{O}(2)-\mathrm{H}(2)$ & 0.8400 \\
$\mathrm{O}(3)-\mathrm{C}(24)$ & $1.408(8)$ \\
$\mathrm{O}(3)-\mathrm{C}(5)$ & $1.418(7)$ \\
$\mathrm{O}(4)-\mathrm{C}(10)$ & $1.227(6)$ \\
$\mathrm{O}(5)-\mathrm{C}(14)$ & $1.366(6)$ \\
$\mathrm{O}(5)-\mathrm{C}(26)$ & $1.449(7)$ \\
$\mathrm{O}(6)-\mathrm{C}(13)$ & $1.224(6)$ \\
$\mathrm{O}(7)-\mathrm{C}(15)$ & $1.223(6)$ \\
$\mathrm{O}(8)-\mathrm{C}(28)$ & $1.424(7)$ \\
$\mathrm{O}(8)-\mathrm{C}(20)$ & $1.432(6)$ \\
$\mathrm{O}(9)-\mathrm{C}(29)$ & $1.363(7)$ \\
$\mathrm{O}(9)-\mathrm{C}(21)$ & $1.463(6)$ \\
$\mathrm{O}(10)-\mathrm{C}(29)$ & $1.214(7)$ \\
$\mathrm{N}(1)-\mathrm{C}(15)$ & $1.363(7)$ \\
$\mathrm{N}(1)-\mathrm{C}(11)$ & $1.392(6)$ \\
$\mathrm{N}(1)-\mathrm{H}(1)$ & 0.8800 \\
$\mathrm{~N}(2)-\mathrm{C}(29)$ & $1.338(7)$ \\
$\mathrm{N}(2)-\mathrm{H}(2 \mathrm{~A})$ & 0.8800 \\
&
\end{tabular}




\begin{tabular}{|c|c|}
\hline $\mathrm{N}(2)-\mathrm{H}(2 \mathrm{~B})$ & 0.8800 \\
\hline$C(1)-C(2)$ & $1.346(7)$ \\
\hline$C(1)-C(22)$ & $1.487(8)$ \\
\hline$C(1)-C(21)$ & $1.510(8)$ \\
\hline$C(2)-C(3)$ & $1.491(8)$ \\
\hline $\mathrm{C}(2)-\mathrm{H}(2 \mathrm{C})$ & 0.9500 \\
\hline$C(3)-C(23)$ & $1.542(8)$ \\
\hline$C(3)-C(4)$ & $1.545(7)$ \\
\hline $\mathrm{C}(3)-\mathrm{H}(3)$ & 1.0000 \\
\hline$C(4)-C(5)$ & $1.540(8)$ \\
\hline $\mathrm{C}(4)-\mathrm{H}(4)$ & 1.0000 \\
\hline$C(5)-C(6)$ & $1.529(8)$ \\
\hline $\mathrm{C}(5)-\mathrm{H}(5)$ & 1.0000 \\
\hline$C(6)-C(7)$ & $1.538(8)$ \\
\hline $\mathrm{C}(6)-\mathrm{H}(6 \mathrm{~A})$ & 0.9900 \\
\hline $\mathrm{C}(6)-\mathrm{H}(6 \mathrm{~B})$ & 0.9900 \\
\hline$C(7)-C(25)$ & $1.524(8)$ \\
\hline$C(7)-C(8)$ & $1.539(7)$ \\
\hline $\mathrm{C}(7)-\mathrm{H}(7)$ & 1.0000 \\
\hline$C(8)-C(9)$ & $1.504(7)$ \\
\hline $\mathrm{C}(8)-\mathrm{H}(8 \mathrm{~A})$ & 0.9900 \\
\hline $\mathrm{C}(8)-\mathrm{H}(8 \mathrm{~B})$ & 0.9900 \\
\hline$C(9)-C(14)$ & $1.501(7)$ \\
\hline$C(9)-C(10)$ & $1.505(7)$ \\
\hline$C(10)-C(11)$ & $1.484(8)$ \\
\hline$C(11)-C(12)$ & $1.346(7)$ \\
\hline$C(12)-C(13)$ & $1.472(7)$ \\
\hline $\mathrm{C}(12)-\mathrm{H}(12)$ & 0.9500 \\
\hline$C(13)-C(14)$ & $1.490(8)$ \\
\hline$C(15)-C(16)$ & $1.493(7)$ \\
\hline$C(16)-C(17)$ & $1.329(7)$ \\
\hline$C(16)-C(27)$ & $1.494(8)$ \\
\hline$C(17)-C(18)$ & $1.450(7)$ \\
\hline $\mathrm{C}(17)-\mathrm{H}(17)$ & 0.9500 \\
\hline$C(18)-C(19)$ & $1.339(7)$ \\
\hline $\mathrm{C}(18)-\mathrm{H}(18)$ & 0.9500 \\
\hline
\end{tabular}




$\begin{array}{ll}\mathrm{C}(19)-\mathrm{C}(20) & 1.499(8) \\ \mathrm{C}(19)-\mathrm{H}(19) & 0.9500 \\ \mathrm{C}(20)-\mathrm{C}(21) & 1.516(8) \\ \mathrm{C}(20)-\mathrm{H}(20) & 1.0000 \\ \mathrm{C}(21)-\mathrm{H}(21) & 1.0000 \\ \mathrm{C}(22)-\mathrm{H}(22 \mathrm{~A}) & 0.9800 \\ \mathrm{C}(22)-\mathrm{H}(22 \mathrm{~B}) & 0.9800 \\ \mathrm{C}(22)-\mathrm{H}(22 \mathrm{C}) & 0.9800 \\ \mathrm{C}(23)-\mathrm{H}(23 \mathrm{~A}) & 0.9800 \\ \mathrm{C}(23)-\mathrm{H}(23 \mathrm{~B}) & 0.9800 \\ \mathrm{C}(23)-\mathrm{H}(23 \mathrm{C}) & 0.9800 \\ \mathrm{C}(24)-\mathrm{H}(24 \mathrm{~A}) & 0.9800 \\ \mathrm{C}(24)-\mathrm{H}(24 \mathrm{~B}) & 0.9800 \\ \mathrm{C}(24)-\mathrm{H}(24 \mathrm{C}) & 0.9800 \\ \mathrm{C}(25)-\mathrm{H}(25 \mathrm{~A}) & 0.9800 \\ \mathrm{C}(25)-\mathrm{H}(25 \mathrm{~B}) & 0.9800 \\ \mathrm{C}(25)-\mathrm{H}(25 \mathrm{C}) & 0.9800 \\ \mathrm{C}(26)-\mathrm{H}(26 \mathrm{~A}) & 0.9800 \\ \mathrm{C}(26)-\mathrm{H}(26 \mathrm{~B}) & 0.9800 \\ \mathrm{C}(26)-\mathrm{H}(26 \mathrm{C}) & 0.9800 \\ \mathrm{C}(27)-\mathrm{H}(27 \mathrm{~A}) & 0.9800 \\ \mathrm{C}(27)-\mathrm{H}(27 \mathrm{~B}) & 0.9800 \\ \mathrm{C}(27)-\mathrm{H}(27 \mathrm{C}) & 0.9800 \\ \mathrm{C}(28)-\mathrm{H}(28 \mathrm{~A}) & 0.9800 \\ \mathrm{C}(28)-\mathrm{H}(28 \mathrm{~B}) & 0.9800 \\ \mathrm{C}(28)-\mathrm{H}(28 \mathrm{C}) & 0.9800 \\ \mathrm{C}(14)-\mathrm{O}(1)-\mathrm{C}(9) & 62.0(3) \\ \mathrm{C}(4)-\mathrm{O}(2)-\mathrm{H}(2) & 109.5 \\ \mathrm{C}(24)-\mathrm{O}(3)-\mathrm{C}(5) & 115.4(5) \\ \mathrm{C}(14)-\mathrm{O}(5)-\mathrm{C}(26) & 115.3(4) \\ \mathrm{C}(28)-\mathrm{O}(8)-\mathrm{C}(20) & 112.8(4) \\ \mathrm{C}(29)-\mathrm{O}(9)-\mathrm{C}(21) & 117.0(4) \\ \mathrm{C}(15)-\mathrm{N}(1)-\mathrm{C}(11) & 129.6(4) \\ \mathrm{C}(15)-\mathrm{N}(1)-\mathrm{H}(1) & 115.2 \\ \mathrm{C}(11)-\mathrm{N}(1)-\mathrm{H}(1) & 115.2 \\ & \end{array}$




$\begin{array}{ll}\mathrm{C}(29)-\mathrm{N}(2)-\mathrm{H}(2 \mathrm{~A}) & 120.0 \\ \mathrm{C}(29)-\mathrm{N}(2)-\mathrm{H}(2 \mathrm{~B}) & 120.0 \\ \mathrm{H}(2 \mathrm{~A})-\mathrm{N}(2)-\mathrm{H}(2 \mathrm{~B}) & 120.0 \\ \mathrm{C}(2)-\mathrm{C}(1)-\mathrm{C}(22) & 125.4(6) \\ \mathrm{C}(2)-\mathrm{C}(1)-\mathrm{C}(21) & 115.0(5) \\ \mathrm{C}(22)-\mathrm{C}(1)-\mathrm{C}(21) & 119.4(5) \\ \mathrm{C}(1)-\mathrm{C}(2)-\mathrm{C}(3) & 129.6(5) \\ \mathrm{C}(1)-\mathrm{C}(2)-\mathrm{H}(2 \mathrm{C}) & 115.2 \\ \mathrm{C}(3)-\mathrm{C}(2)-\mathrm{H}(2 \mathrm{C}) & 115.2 \\ \mathrm{C}(2)-\mathrm{C}(3)-\mathrm{C}(23) & 109.5(5) \\ \mathrm{C}(2)-\mathrm{C}(3)-\mathrm{C}(4) & 109.6(4) \\ \mathrm{C}(23)-\mathrm{C}(3)-\mathrm{C}(4) & 112.8(4) \\ \mathrm{C}(2)-\mathrm{C}(3)-\mathrm{H}(3) & 108.3 \\ \mathrm{C}(23)-\mathrm{C}(3)-\mathrm{H}(3) & 108.3 \\ \mathrm{C}(4)-\mathrm{C}(3)-\mathrm{H}(3) & 108.3 \\ \mathrm{O}(2)-\mathrm{C}(4)-\mathrm{C}(5) & 111.5(4) \\ \mathrm{O}(2)-\mathrm{C}(4)-\mathrm{C}(3) & 112.4(4) \\ \mathrm{C}(5)-\mathrm{C}(4)-\mathrm{C}(3) & 113.2(4) \\ \mathrm{O}(2)-\mathrm{C}(4)-\mathrm{H}(4) & 106.4 \\ \mathrm{C}(5)-\mathrm{C}(4)-\mathrm{H}(4) & 106.4 \\ \mathrm{C}(3)-\mathrm{C}(4)-\mathrm{H}(4) & 106.4 \\ \mathrm{O}(3)-\mathrm{C}(5)-\mathrm{C}(6) & 113.4(5) \\ \mathrm{O}(3)-\mathrm{C}(5)-\mathrm{C}(4) & 104.8(4) \\ \mathrm{C}(6)-\mathrm{C}(5)-\mathrm{C}(4) & 114.1(4) \\ \mathrm{O}(3)-\mathrm{C}(5)-\mathrm{H}(5) & 108.1 \\ \mathrm{C}(6)-\mathrm{C}(5)-\mathrm{H}(5) & 108.1 \\ \mathrm{C}(4)-\mathrm{C}(5)-\mathrm{H}(5) & 108.1 \\ \mathrm{C}(5)-\mathrm{C}(6)-\mathrm{C}(7) & 115.7(4) \\ \mathrm{C}(5)-\mathrm{C}(6)-\mathrm{H}(6 \mathrm{~A}) & 108.3 \\ \mathrm{C}(7)-\mathrm{C}(6)-\mathrm{H}(6 \mathrm{~A}) & 108.3 \\ \mathrm{C}(5)-\mathrm{C}(6)-\mathrm{H}(6 \mathrm{~B}) & 108.3 \\ \mathrm{C}(7)-\mathrm{C}(6)-\mathrm{H}(6 \mathrm{~B}) & 108.3 \\ \mathrm{H}(6 \mathrm{~A})-\mathrm{C}(6)-\mathrm{H}(6 \mathrm{~B}) & 107.4 \\ \mathrm{C}(25)-\mathrm{C}(7)-\mathrm{C}(6) & 111.7(5) \\ \mathrm{C}(25)-\mathrm{C}(7)-\mathrm{C}(8) & 108.6(5) \\ \mathrm{C}(6)-\mathrm{C}(7)-\mathrm{C}(8) & 112.0(4) \\ & \end{array}$




$\begin{array}{ll}\mathrm{C}(25)-\mathrm{C}(7)-\mathrm{H}(7) & 108.1 \\ \mathrm{C}(6)-\mathrm{C}(7)-\mathrm{H}(7) & 108.1 \\ \mathrm{C}(8)-\mathrm{C}(7)-\mathrm{H}(7) & 108.1 \\ \mathrm{C}(9)-\mathrm{C}(8)-\mathrm{C}(7) & 118.7(5) \\ \mathrm{C}(9)-\mathrm{C}(8)-\mathrm{H}(8 \mathrm{~A}) & 107.6 \\ \mathrm{C}(7)-\mathrm{C}(8)-\mathrm{H}(8 \mathrm{~A}) & 107.6 \\ \mathrm{C}(9)-\mathrm{C}(8)-\mathrm{H}(8 \mathrm{~B}) & 107.6 \\ \mathrm{C}(7)-\mathrm{C}(8)-\mathrm{H}(8 \mathrm{~B}) & 107.6 \\ \mathrm{H}(8 \mathrm{~A})-\mathrm{C}(8)-\mathrm{H}(8 \mathrm{~B}) & 107.1 \\ \mathrm{O}(1)-\mathrm{C}(9)-\mathrm{C}(14) & 58.0(3) \\ \mathrm{O}(1)-\mathrm{C}(9)-\mathrm{C}(8) & 117.7(4) \\ \mathrm{C}(14)-\mathrm{C}(9)-\mathrm{C}(8) & 121.0(5) \\ \mathrm{O}(1)-\mathrm{C}(9)-\mathrm{C}(10) & 110.8(4) \\ \mathrm{C}(14)-\mathrm{C}(9)-\mathrm{C}(10) & 115.6(4) \\ \mathrm{C}(8)-\mathrm{C}(9)-\mathrm{C}(10) & 118.6(4) \\ \mathrm{O}(4)-\mathrm{C}(10)-\mathrm{C}(11) & 119.2(5) \\ \mathrm{O}(4)-\mathrm{C}(10)-\mathrm{C}(9) & 121.5(5) \\ \mathrm{C}(11)-\mathrm{C}(10)-\mathrm{C}(9) & 119.3(4) \\ \mathrm{C}(12)-\mathrm{C}(11)-\mathrm{N}(1) & 125.7(5) \\ \mathrm{C}(12)-\mathrm{C}(11)-\mathrm{C}(10) & 122.6(5) \\ \mathrm{N}(1)-\mathrm{C}(11)-\mathrm{C}(10) & 111.6(4) \\ \mathrm{C}(11)-\mathrm{C}(12)-\mathrm{C}(13) & 120.6(5) \\ \mathrm{C}(11)-\mathrm{C}(12)-\mathrm{H}(12) & 119.7 \\ \mathrm{C}(13)-\mathrm{C}(12)-\mathrm{H}(12) & 119.7 \\ \mathrm{O}(6)-\mathrm{C}(13)-\mathrm{C}(12) & 120.0(5) \\ \mathrm{O}(6)-\mathrm{C}(13)-\mathrm{C}(14) & 121.1(5) \\ \mathrm{C}(12)-\mathrm{C}(13)-\mathrm{C}(14) & 118.9(4) \\ \mathrm{O}(5)-\mathrm{C}(14)-\mathrm{O}(1) & 116.8(4) \\ \mathrm{O}(5)-\mathrm{C}(14)-\mathrm{C}(13) & 117.5(4) \\ \mathrm{O}(1)-\mathrm{C}(14)-\mathrm{C}(13) & 111.8(4) \\ \mathrm{O}(5)-\mathrm{C}(14)-\mathrm{C}(9) & 117.3(4) \\ \mathrm{O}(1)-\mathrm{C}(14)-\mathrm{C}(9) & 60.0(3) \\ \mathrm{C}(13)-\mathrm{C}(14)-\mathrm{C}(9) & 119.9(4) \\ \mathrm{O}(7)-\mathrm{C}(15)-\mathrm{N}(1) & 121.9(4) \\ \mathrm{O}(7)-\mathrm{C}(15)-\mathrm{C}(16) & 121.8(5) \\ \mathrm{N}(1)-\mathrm{C}(15)-\mathrm{C}(16) & 116.3(4) \\ & \end{array}$




\begin{tabular}{|c|c|}
\hline$C(17)-C(16)-C(15)$ & $120.0(5)$ \\
\hline$C(17)-C(16)-C(27)$ & $124.5(5)$ \\
\hline$C(15)-C(16)-C(27)$ & $115.3(4)$ \\
\hline$C(16)-C(17)-C(18)$ & $127.6(5)$ \\
\hline $\mathrm{C}(16)-\mathrm{C}(17)-\mathrm{H}(17)$ & 116.2 \\
\hline $\mathrm{C}(18)-\mathrm{C}(17)-\mathrm{H}(17)$ & 116.2 \\
\hline$C(19)-C(18)-C(17)$ & $124.1(5)$ \\
\hline $\mathrm{C}(19)-\mathrm{C}(18)-\mathrm{H}(18)$ & 118.0 \\
\hline $\mathrm{C}(17)-\mathrm{C}(18)-\mathrm{H}(18)$ & 118.0 \\
\hline$C(18)-C(19)-C(20)$ & $125.7(5)$ \\
\hline $\mathrm{C}(18)-\mathrm{C}(19)-\mathrm{H}(19)$ & 117.2 \\
\hline $\mathrm{C}(20)-\mathrm{C}(19)-\mathrm{H}(19)$ & 117.2 \\
\hline $\mathrm{O}(8)-\mathrm{C}(20)-\mathrm{C}(19)$ & $110.6(4)$ \\
\hline $\mathrm{O}(8)-\mathrm{C}(20)-\mathrm{C}(21)$ & $108.8(4)$ \\
\hline$C(19)-C(20)-C(21)$ & $113.3(4)$ \\
\hline $\mathrm{O}(8)-\mathrm{C}(20)-\mathrm{H}(20)$ & 108.0 \\
\hline $\mathrm{C}(19)-\mathrm{C}(20)-\mathrm{H}(20)$ & 108.0 \\
\hline $\mathrm{C}(21)-\mathrm{C}(20)-\mathrm{H}(20)$ & 108.0 \\
\hline $\mathrm{O}(9)-\mathrm{C}(21)-\mathrm{C}(1)$ & $111.3(4)$ \\
\hline $\mathrm{O}(9)-\mathrm{C}(21)-\mathrm{C}(20)$ & $106.6(4)$ \\
\hline$C(1)-C(21)-C(20)$ & $111.5(4)$ \\
\hline $\mathrm{O}(9)-\mathrm{C}(21)-\mathrm{H}(21)$ & 109.1 \\
\hline $\mathrm{C}(1)-\mathrm{C}(21)-\mathrm{H}(21)$ & 109.1 \\
\hline $\mathrm{C}(20)-\mathrm{C}(21)-\mathrm{H}(21)$ & 109.1 \\
\hline $\mathrm{C}(1)-\mathrm{C}(22)-\mathrm{H}(22 \mathrm{~A})$ & 109.5 \\
\hline $\mathrm{C}(1)-\mathrm{C}(22)-\mathrm{H}(22 \mathrm{~B})$ & 109.5 \\
\hline $\mathrm{H}(22 \mathrm{~A})-\mathrm{C}(22)-\mathrm{H}(22 \mathrm{~B})$ & 109.5 \\
\hline$C(1)-C(22)-H(22 C)$ & 109.5 \\
\hline$H(22 A)-C(22)-H(22 C)$ & 109.5 \\
\hline $\mathrm{H}(22 \mathrm{~B})-\mathrm{C}(22)-\mathrm{H}(22 \mathrm{C})$ & 109.5 \\
\hline$C(3)-C(23)-H(23 A)$ & 109.5 \\
\hline $\mathrm{C}(3)-\mathrm{C}(23)-\mathrm{H}(23 \mathrm{~B})$ & 109.5 \\
\hline $\mathrm{H}(23 \mathrm{~A})-\mathrm{C}(23)-\mathrm{H}(23 \mathrm{~B})$ & 109.5 \\
\hline$C(3)-C(23)-H(23 C)$ & 109.5 \\
\hline$H(23 A)-C(23)-H(23 C)$ & 109.5 \\
\hline$H(23 B)-C(23)-H(23 C)$ & 109.5 \\
\hline
\end{tabular}




\begin{tabular}{ll}
$\mathrm{O}(3)-\mathrm{C}(24)-\mathrm{H}(24 \mathrm{~A})$ & 109.5 \\
$\mathrm{O}(3)-\mathrm{C}(24)-\mathrm{H}(24 \mathrm{~B})$ & 109.5 \\
$\mathrm{H}(24 \mathrm{~A})-\mathrm{C}(24)-\mathrm{H}(24 \mathrm{~B})$ & 109.5 \\
$\mathrm{O}(3)-\mathrm{C}(24)-\mathrm{H}(24 \mathrm{C})$ & 109.5 \\
$\mathrm{H}(24 \mathrm{~A})-\mathrm{C}(24)-\mathrm{H}(24 \mathrm{C})$ & 109.5 \\
$\mathrm{H}(24 \mathrm{~B})-\mathrm{C}(24)-\mathrm{H}(24 \mathrm{C})$ & 109.5 \\
$\mathrm{C}(7)-\mathrm{C}(25)-\mathrm{H}(25 \mathrm{~A})$ & 109.5 \\
$\mathrm{C}(7)-\mathrm{C}(25)-\mathrm{H}(25 \mathrm{~B})$ & 109.5 \\
$\mathrm{H}(25 \mathrm{~A})-\mathrm{C}(25)-\mathrm{H}(25 \mathrm{~B})$ & 109.5 \\
$\mathrm{C}(7)-\mathrm{C}(25)-\mathrm{H}(25 \mathrm{C})$ & 109.5 \\
$\mathrm{H}(25 \mathrm{~A})-\mathrm{C}(25)-\mathrm{H}(25 \mathrm{C})$ & 109.5 \\
$\mathrm{H}(25 \mathrm{~B})-\mathrm{C}(25)-\mathrm{H}(25 \mathrm{C})$ & 109.5 \\
$\mathrm{O}(5)-\mathrm{C}(26)-\mathrm{H}(26 \mathrm{~A})$ & 109.5 \\
$\mathrm{O}(5)-\mathrm{C}(26)-\mathrm{H}(26 \mathrm{~B})$ & 109.5 \\
$\mathrm{H}(26 \mathrm{~A})-\mathrm{C}(26)-\mathrm{H}(26 \mathrm{~B})$ & 109.5 \\
$\mathrm{O}(5)-\mathrm{C}(26)-\mathrm{H}(26 \mathrm{C})$ & 109.5 \\
$\mathrm{H}(26 \mathrm{~A})-\mathrm{C}(26)-\mathrm{H}(26 \mathrm{C})$ & 109.5 \\
$\mathrm{H}(26 \mathrm{~B})-\mathrm{C}(26)-\mathrm{H}(26 \mathrm{C})$ & 109.5 \\
$\mathrm{C}(16)-\mathrm{C}(27)-\mathrm{H}(27 \mathrm{~A})$ & 109.5 \\
$\mathrm{C}(16)-\mathrm{C}(27)-\mathrm{H}(27 \mathrm{~B})$ & 109.5 \\
$\mathrm{H}(27 \mathrm{~A})-\mathrm{C}(27)-\mathrm{H}(27 \mathrm{~B})$ & 109.5 \\
$\mathrm{C}(16)-\mathrm{C}(27)-\mathrm{H}(27 \mathrm{C})$ & 109.5 \\
$\mathrm{H}(27 \mathrm{~A})-\mathrm{C}(27)-\mathrm{H}(27 \mathrm{C})$ & 109.5 \\
$\mathrm{H}(27 \mathrm{~B})-\mathrm{C}(27)-\mathrm{H}(27 \mathrm{C})$ & 109.5 \\
$\mathrm{O}(8)-\mathrm{C}(28)-\mathrm{H}(28 \mathrm{~A})$ & 109.5 \\
$\mathrm{O}(8)-\mathrm{C}(28)-\mathrm{H}(28 \mathrm{~B})$ & 109.5 \\
$\mathrm{H}(28 \mathrm{~A})-\mathrm{C}(28)-\mathrm{H}(28 \mathrm{~B})$ & 109.5 \\
$\mathrm{O}(8)-\mathrm{C}(28)-\mathrm{H}(28 \mathrm{C})$ & 109.5 \\
$\mathrm{H}(28 \mathrm{~A})-\mathrm{C}(28)-\mathrm{H}(28 \mathrm{C})$ & 109.5 \\
$\mathrm{H}(28 \mathrm{~B})-\mathrm{C}(28)-\mathrm{H}(28 \mathrm{C})$ & 109.5 \\
$\mathrm{O}(10)-\mathrm{C}(29)-\mathrm{N}(2)$ & $126.6(5)$ \\
$\mathrm{O}(10)-\mathrm{C}(29)-\mathrm{O}(9)$ & $123.3(5)$ \\
$\mathrm{N}(2)-\mathrm{C}(29)-\mathrm{O}(9)$ & $110.0(5)$ \\
& \\
\hline
\end{tabular}

Symmetry transformations used to generate equivalent atoms: 
Table S11. Anisotropic displacement parameters $\left(\AA^{2} \times 10^{3}\right)$ for $\mathbf{C}$. The anisotropic displacement factor exponent takes the form: $-2 p^{2}\left[h^{2} a^{* 2} U^{11}+\ldots+2 h k a^{*} b^{*} U^{12}\right]$

\begin{tabular}{|c|c|c|c|c|c|c|}
\hline & $u^{11}$ & $u^{22}$ & U33 & $u^{23}$ & $U^{13}$ & $U^{12}$ \\
\hline $\mathrm{O}(1)$ & $46(2)$ & $37(2)$ & $42(2)$ & $0(2)$ & $-2(2)$ & $-2(2)$ \\
\hline $\mathrm{O}(2)$ & $47(2)$ & $47(2)$ & $41(2)$ & $-1(2)$ & $5(2)$ & $-2(2)$ \\
\hline $\mathrm{O}(3)$ & $51(2)$ & $83(3)$ & $37(2)$ & $5(2)$ & $7(2)$ & $8(2)$ \\
\hline $\mathrm{O}(4)$ & $42(2)$ & $46(2)$ & $44(2)$ & $-4(2)$ & $1(2)$ & $-1(2)$ \\
\hline $\mathrm{O}(5)$ & $38(2)$ & $52(2)$ & $43(2)$ & $-1(2)$ & $-3(2)$ & $0(2)$ \\
\hline $\mathrm{O}(6)$ & $47(2)$ & $73(3)$ & $42(2)$ & $7(2)$ & $-4(2)$ & $-2(2)$ \\
\hline $\mathrm{O}(7)$ & $39(2)$ & $47(2)$ & $39(2)$ & $-2(2)$ & $-1(2)$ & $5(2)$ \\
\hline $\mathrm{O}(8)$ & $47(2)$ & $37(2)$ & $48(2)$ & $0(2)$ & $-8(2)$ & $2(2)$ \\
\hline $\mathrm{O}(9)$ & $47(2)$ & $36(2)$ & $42(2)$ & $0(2)$ & $-13(2)$ & $2(2)$ \\
\hline$O(10)$ & $62(3)$ & $44(2)$ & $60(2)$ & $-5(2)$ & $-18(2)$ & $10(2)$ \\
\hline$N(1)$ & $36(2)$ & $46(2)$ & $36(2)$ & $0(2)$ & $-3(2)$ & $-2(2)$ \\
\hline$N(2)$ & $44(3)$ & $43(2)$ & $46(3)$ & $7(2)$ & $-9(2)$ & $2(2)$ \\
\hline$C(1)$ & $52(3)$ & $33(3)$ & $38(3)$ & $6(2)$ & $-5(2)$ & $2(2)$ \\
\hline$C(2)$ & $51(3)$ & $41(3)$ & $35(3)$ & $-2(2)$ & $-2(2)$ & $1(3)$ \\
\hline$C(3)$ & $43(3)$ & $46(3)$ & $36(3)$ & $1(2)$ & $-2(2)$ & $-1(2)$ \\
\hline$C(4)$ & $45(3)$ & $38(3)$ & $41(3)$ & $1(2)$ & $2(2)$ & $-2(2)$ \\
\hline$C(5)$ & $47(3)$ & $41(3)$ & $41(3)$ & $3(2)$ & $-1(2)$ & $-3(2)$ \\
\hline$C(6)$ & $41(3)$ & $45(3)$ & $42(3)$ & $0(2)$ & $-3(2)$ & $-7(2)$ \\
\hline$C(7)$ & $31(3)$ & $53(3)$ & $43(3)$ & $-1(3)$ & $-1(2)$ & $-6(2)$ \\
\hline$C(8)$ & $41(3)$ & $40(3)$ & $42(3)$ & $1(2)$ & $0(2)$ & $-1(2)$ \\
\hline$C(9)$ & $43(3)$ & $35(3)$ & $40(3)$ & $5(2)$ & $3(2)$ & $0(2)$ \\
\hline$C(10)$ & $47(3)$ & $27(2)$ & $42(3)$ & $3(2)$ & $-6(2)$ & $3(2)$ \\
\hline$C(11)$ & $39(3)$ & 33(3) & $43(3)$ & $6(2)$ & $-1(2)$ & $2(2)$ \\
\hline$C(12)$ & $45(3)$ & $42(3)$ & $43(3)$ & 2(2) & $5(2)$ & $0(2)$ \\
\hline$C(13)$ & $48(3)$ & $36(3)$ & $42(3)$ & $1(2)$ & $-4(2)$ & $-1(2)$ \\
\hline$C(14)$ & $43(3)$ & $32(3)$ & $45(3)$ & $-1(2)$ & $-6(2)$ & $-1(2)$ \\
\hline$C(15)$ & $37(3)$ & $31(3)$ & $45(3)$ & $4(2)$ & $-1(2)$ & $0(2)$ \\
\hline$C(16)$ & $39(3)$ & $31(3)$ & $43(3)$ & $-3(2)$ & $-2(2)$ & $2(2)$ \\
\hline$C(17)$ & $36(3)$ & $41(3)$ & $52(3)$ & $-1(2)$ & $1(2)$ & $3(2)$ \\
\hline$C(18)$ & $34(3)$ & $45(3)$ & $51(3)$ & $3(2)$ & $0(2)$ & $3(2)$ \\
\hline$C(19)$ & $38(3)$ & $44(3)$ & $44(3)$ & $1(2)$ & $-8(2)$ & $5(2)$ \\
\hline
\end{tabular}




$\begin{array}{llllllc}\mathrm{C}(20) & 45(3) & 39(3) & 39(3) & -2(2) & -11(2) & 3(2) \\ \mathrm{C}(21) & 42(3) & 41(3) & 36(3) & 0(2) & -10(2) & 5(2) \\ \mathrm{C}(22) & 58(4) & 88(5) & 40(3) & -7(3) & 3(3) & -15(4) \\ \mathrm{C}(23) & 42(3) & 46(3) & 58(3) & 2(3) & 1(3) & -3(3) \\ \mathrm{C}(24) & 61(4) & 102(6) & 55(4) & 24(4) & 22(3) & 24(4) \\ \mathrm{C}(25) & 49(3) & 53(3) & 45(3) & -4(3) & 4(2) & -4(3) \\ \mathrm{C}(26) & 46(3) & 58(4) & 51(3) & -8(3) & -6(3) & -4(3) \\ \mathrm{C}(27) & 49(3) & 40(3) & 43(3) & 0(2) & -3(2) & 1(2) \\ \mathrm{C}(28) & 66(4) & 37(3) & 71(4) & 3(3) & -11(3) & 7(3) \\ \mathrm{C}(29) & 48(3) & 38(3) & 45(3) & 7(2) & -7(2) & -5(2)\end{array}$

Table S12. Hydrogen coordinates ( x 104) and isotropic displacement parameters $\left(\AA^{2} \times 10^{3}\right)$ for $\mathbf{C}$.

\begin{tabular}{|c|c|c|c|c|}
\hline & $x$ & $y$ & $z$ & $\mathrm{U}(\mathrm{eq})$ \\
\hline $\mathrm{H}(2)$ & 4068 & 3253 & 6800 & 68 \\
\hline$H(1)$ & 5967 & 7724 & 5954 & 48 \\
\hline$H(2 A)$ & 10586 & 2366 & 10410 & 55 \\
\hline $\mathrm{H}(2 \mathrm{~B})$ & 10063 & 4127 & 10557 & 55 \\
\hline $\mathrm{H}(2 \mathrm{C})$ & 6147 & 2889 & 7907 & 52 \\
\hline $\mathrm{H}(3)$ & 4617 & 3236 & 9033 & 51 \\
\hline $\mathrm{H}(4)$ & 4351 & 5552 & 8057 & 50 \\
\hline $\mathrm{H}(5)$ & 2636 & 3051 & 7846 & 53 \\
\hline$H(6 A)$ & 1414 & 5168 & 7231 & 52 \\
\hline $\mathrm{H}(6 \mathrm{~B})$ & 2287 & 4825 & 6628 & 52 \\
\hline $\mathrm{H}(7)$ & 3183 & 7530 & 7382 & 52 \\
\hline$H(8 A)$ & 1411 & 7820 & 6017 & 50 \\
\hline $\mathrm{H}(8 \mathrm{~B})$ & 2250 & 9371 & 6286 & 50 \\
\hline $\mathrm{H}(12)$ & 4695 & 6980 & 3877 & 52 \\
\hline $\mathrm{H}(17)$ & 7127 & 6526 & 6598 & 53 \\
\hline $\mathrm{H}(18)$ & 9436 & 6500 & 6817 & 53 \\
\hline$H(19)$ & 9446 & 6048 & 8188 & 52 \\
\hline
\end{tabular}




\begin{tabular}{|c|c|c|c|c|}
\hline $\mathrm{H}(20)$ & 7136 & 5930 & 7796 & 52 \\
\hline$H(21)$ & 7915 & 3189 & 8352 & 50 \\
\hline$H(22 A)$ & 5790 & 4381 & 9933 & 94 \\
\hline $\mathrm{H}(22 \mathrm{~B})$ & 6827 & 5627 & 9994 & 94 \\
\hline $\mathrm{H}(22 \mathrm{C})$ & 6959 & 3618 & 10298 & 94 \\
\hline $\mathrm{H}(23 \mathrm{~A})$ & 4498 & 855 & 7622 & 74 \\
\hline $\mathrm{H}(23 \mathrm{~B})$ & 3669 & 896 & 8284 & 74 \\
\hline $\mathrm{H}(23 \mathrm{C})$ & 4888 & 280 & 8585 & 74 \\
\hline $\mathrm{H}(24 \mathrm{~A})$ & 1224 & 4718 & 8684 & 107 \\
\hline $\mathrm{H}(24 \mathrm{~B})$ & 1911 & 4772 & 9617 & 107 \\
\hline $\mathrm{H}(24 \mathrm{C})$ & 1897 & 3023 & 9056 & 107 \\
\hline $\mathrm{H}(25 \mathrm{~A})$ & 968 & 8196 & 7486 & 74 \\
\hline $\mathrm{H}(25 \mathrm{~B})$ & 1916 & 9616 & 7689 & 74 \\
\hline $\mathrm{H}(25 \mathrm{C})$ & 1895 & 7931 & 8287 & 74 \\
\hline$H(26 A)$ & 971 & 6668 & 3291 & 80 \\
\hline$H(26 B)$ & -63 & 6843 & 3751 & 80 \\
\hline $\mathrm{H}(26 \mathrm{C})$ & 787 & 5287 & 4010 & 80 \\
\hline$H(27 A)$ & 8913 & 5823 & 5007 & 68 \\
\hline $\mathrm{H}(27 \mathrm{~B})$ & 9250 & 7678 & 5449 & 68 \\
\hline $\mathrm{H}(27 \mathrm{C})$ & 8451 & 7617 & 4563 & 68 \\
\hline $\mathrm{H}(28 \mathrm{~A})$ & 7757 & 9281 & 8002 & 91 \\
\hline $\mathrm{H}(28 \mathrm{~B})$ & 7229 & 9828 & 8809 & 91 \\
\hline $\mathrm{H}(28 \mathrm{C})$ & 6559 & 8659 & 8074 & 91 \\
\hline
\end{tabular}

Table S13. Torsion angles $\left[^{\circ}\right]$ for $\mathbf{C}$.

\begin{tabular}{lc}
\hline$C(22)-C(1)-C(2)-C(3)$ & $3.8(10)$ \\
$C(21)-C(1)-C(2)-C(3)$ & $-171.4(5)$ \\
$C(1)-C(2)-C(3)-C(23)$ & $-126.6(6)$ \\
$C(1)-C(2)-C(3)-C(4)$ & $109.1(6)$ \\
$C(2)-C(3)-C(4)-O(2)$ & $57.2(6)$ \\
$C(23)-C(3)-C(4)-O(2)$ & $-65.0(6)$ \\
$C(2)-C(3)-C(4)-C(5)$ & $-175.4(4)$ \\
$C(23)-C(3)-C(4)-C(5)$ & $62.3(6)$
\end{tabular}




\begin{tabular}{|c|c|}
\hline $\mathrm{C}(24)-\mathrm{O}(3)-\mathrm{C}(5)-\mathrm{C}(6)$ & $82.0(6)$ \\
\hline$C(24)-O(3)-C(5)-C(4)$ & $-152.9(5)$ \\
\hline $\mathrm{O}(2)-\mathrm{C}(4)-\mathrm{C}(5)-\mathrm{O}(3)$ & $-164.3(4)$ \\
\hline$C(3)-C(4)-C(5)-O(3)$ & $67.9(6)$ \\
\hline $\mathrm{O}(2)-\mathrm{C}(4)-\mathrm{C}(5)-\mathrm{C}(6)$ & $-39.6(6)$ \\
\hline$C(3)-C(4)-C(5)-C(6)$ & $-167.5(4)$ \\
\hline$O(3)-C(5)-C(6)-C(7)$ & $58.2(6)$ \\
\hline$C(4)-C(5)-C(6)-C(7)$ & $-61.7(7)$ \\
\hline$C(5)-C(6)-C(7)-C(25)$ & $-88.2(6)$ \\
\hline$C(5)-C(6)-C(7)-C(8)$ & $149.7(5)$ \\
\hline$C(25)-C(7)-C(8)-C(9)$ & $172.0(5)$ \\
\hline$C(6)-C(7)-C(8)-C(9)$ & $-64.2(6)$ \\
\hline$C(14)-O(1)-C(9)-C(8)$ & $111.0(5)$ \\
\hline$C(14)-O(1)-C(9)-C(10)$ & $-107.9(4)$ \\
\hline $\mathrm{C}(7)-\mathrm{C}(8)-\mathrm{C}(9)-\mathrm{O}(1)$ & $77.4(6)$ \\
\hline$C(7)-C(8)-C(9)-C(14)$ & $144.9(5)$ \\
\hline$C(7)-C(8)-C(9)-C(10)$ & $-60.7(7)$ \\
\hline $\mathrm{O}(1)-\mathrm{C}(9)-\mathrm{C}(10)-\mathrm{O}(4)$ & $-131.0(5)$ \\
\hline$C(14)-C(9)-C(10)-O(4)$ & $165.6(5)$ \\
\hline$C(8)-C(9)-C(10)-O(4)$ & $9.7(7)$ \\
\hline$O(1)-C(9)-C(10)-C(11)$ & $49.5(6)$ \\
\hline$C(14)-C(9)-C(10)-C(11)$ & $-14.0(7)$ \\
\hline$C(8)-C(9)-C(10)-C(11)$ & $-169.8(5)$ \\
\hline$C(15)-N(1)-C(11)-C(12)$ & $-8.5(9)$ \\
\hline$C(15)-N(1)-C(11)-C(10)$ & $174.4(5)$ \\
\hline$O(4)-C(10)-C(11)-C(12)$ & $-164.9(5)$ \\
\hline$C(9)-C(10)-C(11)-C(12)$ & $14.7(7)$ \\
\hline $\mathrm{O}(4)-\mathrm{C}(10)-\mathrm{C}(11)-\mathrm{N}(1)$ & $12.3(7)$ \\
\hline$C(9)-C(10)-C(11)-N(1)$ & $-168.2(4)$ \\
\hline$N(1)-C(11)-C(12)-C(13)$ & $-176.8(5)$ \\
\hline$C(10)-C(11)-C(12)-C(13)$ & $0.0(8)$ \\
\hline$C(11)-C(12)-C(13)-O(6)$ & $165.4(5)$ \\
\hline$C(11)-C(12)-C(13)-C(14)$ & $-14.2(8)$ \\
\hline$C(26)-O(5)-C(14)-O(1)$ & $-74.7(6)$ \\
\hline$C(26)-O(5)-C(14)-C(13)$ & $62.6(6)$ \\
\hline $\mathrm{C}(26)-\mathrm{O}(5)-\mathrm{C}(14)-\mathrm{C}(9)$ & $-143.0(5)$ \\
\hline
\end{tabular}




\begin{tabular}{|c|c|}
\hline $\mathrm{C}(9)-\mathrm{O}(1)-\mathrm{C}(14)-\mathrm{O}(5)$ & $-107.5(5)$ \\
\hline$C(9)-O(1)-C(14)-C(13)$ & $112.9(5)$ \\
\hline $\mathrm{O}(6)-\mathrm{C}(13)-\mathrm{C}(14)-\mathrm{O}(5)$ & $-12.0(8)$ \\
\hline$C(12)-C(13)-C(14)-O(5)$ & $167.6(5)$ \\
\hline$O(6)-C(13)-C(14)-O(1)$ & $127.3(5)$ \\
\hline$C(12)-C(13)-C(14)-O(1)$ & $-53.1(6)$ \\
\hline$O(6)-C(13)-C(14)-C(9)$ & $-165.8(5)$ \\
\hline$C(12)-C(13)-C(14)-C(9)$ & $13.8(7)$ \\
\hline $\mathrm{O}(1)-\mathrm{C}(9)-\mathrm{C}(14)-\mathrm{O}(5)$ & $106.8(5)$ \\
\hline$C(8)-C(9)-C(14)-O(5)$ & $1.5(7)$ \\
\hline$C(10)-C(9)-C(14)-O(5)$ & $-153.7(4)$ \\
\hline$C(8)-C(9)-C(14)-O(1)$ & $-105.3(5)$ \\
\hline$C(10)-C(9)-C(14)-O(1)$ & $99.5(5)$ \\
\hline$O(1)-C(9)-C(14)-C(13)$ & $-99.4(5)$ \\
\hline$C(8)-C(9)-C(14)-C(13)$ & $155.4(5)$ \\
\hline$C(10)-C(9)-C(14)-C(13)$ & $0.2(7)$ \\
\hline $\mathrm{C}(11)-\mathrm{N}(1)-\mathrm{C}(15)-\mathrm{O}(7)$ & $6.4(9)$ \\
\hline$C(11)-N(1)-C(15)-C(16)$ & $-171.4(5)$ \\
\hline$O(7)-C(15)-C(16)-C(17)$ & $-156.0(5)$ \\
\hline$N(1)-C(15)-C(16)-C(17)$ & $21.8(7)$ \\
\hline$O(7)-C(15)-C(16)-C(27)$ & $19.0(7)$ \\
\hline$N(1)-C(15)-C(16)-C(27)$ & $-163.2(4)$ \\
\hline$C(15)-C(16)-C(17)-C(18)$ & $178.0(5)$ \\
\hline$C(27)-C(16)-C(17)-C(18)$ & $3.5(9)$ \\
\hline$C(16)-C(17)-C(18)-C(19)$ & $179.6(6)$ \\
\hline$C(17)-C(18)-C(19)-C(20)$ & $1.9(9)$ \\
\hline$C(28)-O(8)-C(20)-C(19)$ & $78.4(6)$ \\
\hline$C(28)-O(8)-C(20)-C(21)$ & $-156.5(5)$ \\
\hline$C(18)-C(19)-C(20)-O(8)$ & $-113.9(6)$ \\
\hline$C(18)-C(19)-C(20)-C(21)$ & $123.6(6)$ \\
\hline$C(29)-O(9)-C(21)-C(1)$ & 109.3(5) \\
\hline$C(29)-O(9)-C(21)-C(20)$ & $-128.9(5)$ \\
\hline$C(2)-C(1)-C(21)-O(9)$ & $-160.4(5)$ \\
\hline$C(22)-C(1)-C(21)-O(9)$ & 24.1(7) \\
\hline$C(2)-C(1)-C(21)-C(20)$ & $80.7(6)$ \\
\hline$C(22)-C(1)-C(21)-C(20)$ & $-94.8(6)$ \\
\hline
\end{tabular}


$\mathrm{O}(8)-\mathrm{C}(20)-\mathrm{C}(21)-\mathrm{O}(9)$

$\mathrm{C}(19)-\mathrm{C}(20)-\mathrm{C}(21)-\mathrm{O}(9)$

$\mathrm{O}(8)-\mathrm{C}(20)-\mathrm{C}(21)-\mathrm{C}(1)$

$\mathrm{C}(19)-\mathrm{C}(20)-\mathrm{C}(21)-\mathrm{C}(1)$

$\mathrm{C}(21)-\mathrm{O}(9)-\mathrm{C}(29)-\mathrm{O}(10)$

$\mathrm{C}(21)-\mathrm{O}(9)-\mathrm{C}(29)-\mathrm{N}(2)$
$-56.1(5)$

$67.4(5)$

$65.6(5)$

$-170.9(4)$

$1.0(8)$

$-178.1(4)$

Symmetry transformations used to generate equivalent atoms:

Table S14. Hydrogen bonds for C [Å and ${ }^{\circ}$.

\begin{tabular}{lcccc}
\hline $\mathrm{D}-\mathrm{H} \ldots \mathrm{A}$ & $\mathrm{d}(\mathrm{D}-\mathrm{H})$ & $\mathrm{d}(\mathrm{H} \ldots \mathrm{A})$ & $\mathrm{d}(\mathrm{D} \ldots \mathrm{A})$ & $<(\mathrm{DHA})$ \\
\hline $\mathrm{O}(2)-\mathrm{H}(2) \ldots \mathrm{O}(7) \# 1$ & 0.84 & 2.08 & $2.830(5)$ & 148.9 \\
$\mathrm{~N}(2)-\mathrm{H}(2 \mathrm{~A}) \ldots \mathrm{O}(8) \# 2$ & 0.88 & 2.31 & $3.008(6)$ & 136.4 \\
$\mathrm{~N}(2)-\mathrm{H}(2 \mathrm{~A}) \ldots \mathrm{O}(9) \# 2$ & 0.88 & 2.52 & $3.343(6)$ & 155.1 \\
$\mathrm{~N}(2)-\mathrm{H}(2 \mathrm{~B}) \ldots \mathrm{O}(10) \# 3$ & 0.88 & 2.11 & $2.902(6)$ & 148.5 \\
\hline
\end{tabular}

Symmetry transformations used to generate equivalent atoms:

$\# 1-x+1, y-1 / 2,-z+1 \quad \# 2-x+2, y-1 / 2,-z+2 \quad \# 3-x+2, y+1 / 2,-z+2$ 
Refinement details for $\mathbf{D}$ :

The model of atoms $\mathrm{H} 1, \mathrm{H} 2 \mathrm{~A}$, and $\mathrm{H} 2 \mathrm{~B}$ were placed based on the Fourier difference map and freely refined.

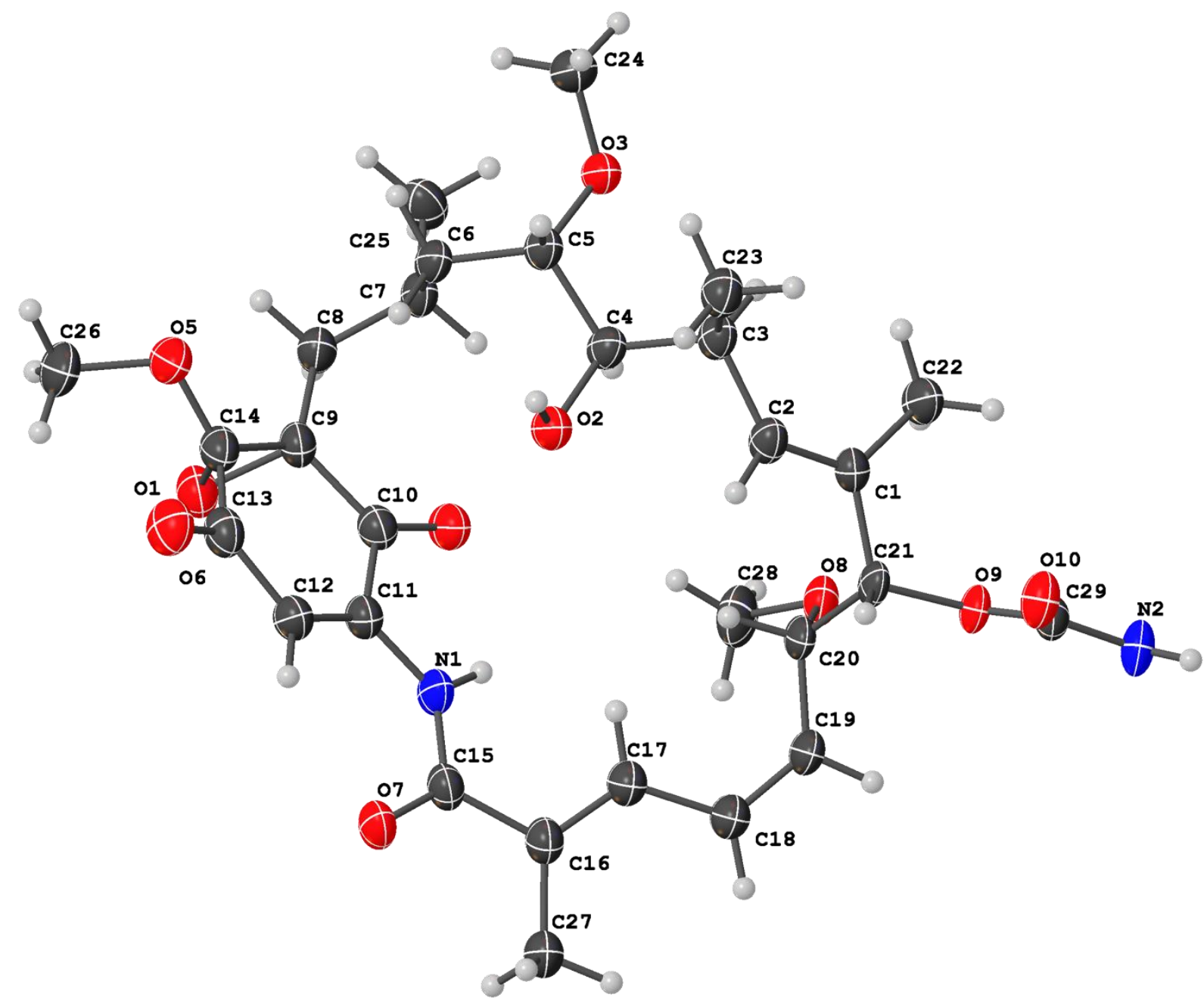

Figure S3. The complete numbering scheme of D with $50 \%$ thermal ellipsoid probability levels. The hydrogen atoms are shown as circles for clarity.

Table S15. Atomic coordinates $\left(\times 10^{4}\right)$ and equivalent isotropic displacement parameters $\left(\AA^{2} \times 10^{3}\right)$ for $\mathbf{D}$. $U(\mathrm{eq})$ is defined as one third of the trace of the orthogonalized $U^{i j}$ tensor.

\begin{tabular}{lrccc}
\hline & $x$ & $y$ & $z$ & $U(e q)$ \\
\hline$O(1)$ & $625(2)$ & $8408(1)$ & $3935(1)$ & $44(1)$ \\
$O(2)$ & $5189(3)$ & $6500(1)$ & $3867(1)$ & $38(1)$
\end{tabular}




\begin{tabular}{|c|c|c|c|c|}
\hline $\mathrm{O}(3)$ & $5140(2)$ & $6125(1)$ & $2120(1)$ & $36(1)$ \\
\hline $\mathrm{O}(4)$ & $1202(2)$ & $6632(1)$ & $4066(1)$ & $39(1)$ \\
\hline $\mathrm{O}(5)$ & $2858(3)$ & $8955(1)$ & $3376(1)$ & $47(1)$ \\
\hline $\mathrm{O}(6)$ & $3984(3)$ & $9133(1)$ & $4643(1)$ & $49(1)$ \\
\hline $\mathrm{O}(7)$ & $2452(3)$ & $7361(1)$ & $6281(1)$ & $40(1)$ \\
\hline $\mathrm{O}(8)$ & $2421(2)$ & $4073(1)$ & $4456(1)$ & $37(1)$ \\
\hline $\mathrm{O}(9)$ & $5162(2)$ & $3362(1)$ & $4768(1)$ & $32(1)$ \\
\hline $\mathrm{O}(10)$ & $7855(2)$ & $3300(1)$ & $5019(1)$ & $42(1)$ \\
\hline $\mathrm{N}(1)$ & 1972(3) & $6855(1)$ & $5286(1)$ & $36(1)$ \\
\hline $\mathrm{N}(2)$ & $6197(3)$ & $2320(1)$ & $5051(1)$ & $40(1)$ \\
\hline$C(1)$ & $5719(3)$ & $4358(1)$ & $3999(1)$ & $31(1)$ \\
\hline $\mathrm{C}(2)$ & $6117(3)$ & $5041(1)$ & $3918(1)$ & $35(1)$ \\
\hline$C(3)$ & $6365(3)$ & $5456(1)$ & $3295(1)$ & $34(1)$ \\
\hline$C(4)$ & $5125(3)$ & $6082(1)$ & $3282(1)$ & $33(1)$ \\
\hline$C(5)$ & $5299(3)$ & $6576(1)$ & $2684(1)$ & $33(1)$ \\
\hline$C(6)$ & $4099(3)$ & $7204(1)$ & $2692(1)$ & $35(1)$ \\
\hline$C(7)$ & $2279(4)$ & $7023(1)$ & $2679(1)$ & $35(1)$ \\
\hline $\mathrm{C}(8)$ & $1234(4)$ & $7617(1)$ & $2985(1)$ & $38(1)$ \\
\hline $\mathrm{C}(9)$ & $1616(3)$ & $7819(1)$ & $3681(1)$ & $35(1)$ \\
\hline$C(10)$ & $1667(3)$ & $7238(1)$ & $4184(1)$ & $35(1)$ \\
\hline$C(11)$ & 2213(3) & $7422(1)$ & $4866(1)$ & $34(1)$ \\
\hline$C(12)$ & $2898(4)$ & $8053(1)$ & $5016(1)$ & $37(1)$ \\
\hline$C(13)$ & $3149(3)$ & $8604(1)$ & $4526(2)$ & $39(1)$ \\
\hline$C(14)$ & $2342(4)$ & $8512(1)$ & $3863(1)$ & $38(1)$ \\
\hline$C(15)$ & $2247(4)$ & $6823(1)$ & $5949(1)$ & $36(1)$ \\
\hline$C(16)$ & $2249(4)$ & $6092(1)$ & $6238(1)$ & $37(1)$ \\
\hline$C(17)$ & $2636(4)$ & $5543(1)$ & $5846(1)$ & $39(1)$ \\
\hline$C(18)$ & $2735(4)$ & $4795(1)$ & $6009(1)$ & $42(1)$ \\
\hline$C(19)$ & $3159(4)$ & $4292(1)$ & $5580(1)$ & $42(1)$ \\
\hline$C(20)$ & $3586(4)$ & $4411(1)$ & $4874(1)$ & $35(1)$ \\
\hline$C(21)$ & $5279(3)$ & $4129(1)$ & $4689(1)$ & $32(1)$ \\
\hline$C(22)$ & $5570(4)$ & $3813(1)$ & $3470(1)$ & $43(1)$ \\
\hline$C(23)$ & $8153(4)$ & $5702(2)$ & $3235(2)$ & $41(1)$ \\
\hline$C(24)$ & $5809(4)$ & $6434(2)$ & $1542(1)$ & $45(1)$ \\
\hline$C(25)$ & $1653(4)$ & $6892(2)$ & $1987(1)$ & $46(1)$ \\
\hline$C(26)$ & $2226(5)$ & $9674(2)$ & $3430(2)$ & $56(1)$ \\
\hline
\end{tabular}




$\begin{array}{lrlll}\mathrm{C}(27) & 1959(4) & 6054(2) & 6960(1) & 46(1) \\ \mathrm{C}(28) & 917(4) & 4457(2) & 4420(2) & 53(1) \\ \mathrm{C}(29) & 6534(3) & 3008(1) & 4957(1) & 32(1)\end{array}$

Table S16. Bond lengths $[\AA ̊ \AA]$ and angles $\left[^{\circ}\right]$ for $\mathbf{D}$.

\begin{tabular}{ll}
\hline $\mathrm{O}(1)-\mathrm{C}(14)$ & $1.427(4)$ \\
$\mathrm{O}(1)-\mathrm{C}(9)$ & $1.466(3)$ \\
$\mathrm{O}(2)-\mathrm{C}(4)$ & $1.431(3)$ \\
$\mathrm{O}(2)-\mathrm{H}(2)$ & $0.96(4)$ \\
$\mathrm{O}(3)-\mathrm{C}(24)$ & $1.425(3)$ \\
$\mathrm{O}(3)-\mathrm{C}(5)$ & $1.436(3)$ \\
$\mathrm{O}(4)-\mathrm{C}(10)$ & $1.222(3)$ \\
$\mathrm{O}(5)-\mathrm{C}(14)$ & $1.363(3)$ \\
$\mathrm{O}(5)-\mathrm{C}(26)$ & $1.450(3)$ \\
$\mathrm{O}(6)-\mathrm{C}(13)$ & $1.228(3)$ \\
$\mathrm{O}(7)-\mathrm{C}(15)$ & $1.228(3)$ \\
$\mathrm{O}(8)-\mathrm{C}(20)$ & $1.428(3)$ \\
$\mathrm{O}(8)-\mathrm{C}(28)$ & $1.429(3)$ \\
$\mathrm{O}(9)-\mathrm{C}(29)$ & $1.362(3)$ \\
$\mathrm{O}(9)-\mathrm{C}(21)$ & $1.451(3)$ \\
$\mathrm{O}(10)-\mathrm{C}(29)$ & $1.220(3)$ \\
$\mathrm{N}(1)-\mathrm{C}(15)$ & $1.377(4)$ \\
$\mathrm{N}(1)-\mathrm{C}(11)$ & $1.379(3)$ \\
$\mathrm{N}(1)-\mathrm{H}(1)$ & $0.90(4)$ \\
$\mathrm{N}(2)-\mathrm{C}(29)$ & $1.333(4)$ \\
$\mathrm{N}(2)-\mathrm{H}(2 \mathrm{~A})$ & $0.93(4)$ \\
$\mathrm{N}(2)-\mathrm{H}(2 \mathrm{~B})$ & $0.89(4)$ \\
$\mathrm{C}(1)-\mathrm{C}(2)$ & $1.333(4)$ \\
$\mathrm{C}(1)-\mathrm{C}(22)$ & $1.493(4)$ \\
$\mathrm{C}(1)-\mathrm{C}(21)$ & $1.519(4)$ \\
$\mathrm{C}(2)-\mathrm{C}(3)$ & $1.506(4)$ \\
$\mathrm{C}(2)-\mathrm{H}(2 \mathrm{C})$ & 0.9500 \\
$\mathrm{C}(3)-\mathrm{C}(23)$ & $1.540(4)$ \\
&
\end{tabular}




$\begin{array}{ll}\mathrm{C}(3)-\mathrm{C}(4) & 1.553(4) \\ \mathrm{C}(3)-\mathrm{H}(3) & 1.0000 \\ \mathrm{C}(4)-\mathrm{C}(5) & 1.540(4) \\ \mathrm{C}(4)-\mathrm{H}(4) & 1.0000 \\ \mathrm{C}(5)-\mathrm{C}(6) & 1.533(4) \\ \mathrm{C}(5)-\mathrm{H}(5) & 1.0000 \\ \mathrm{C}(6)-\mathrm{C}(7) & 1.529(4) \\ \mathrm{C}(6)-\mathrm{H}(6 \mathrm{~A}) & 0.9900 \\ \mathrm{C}(6)-\mathrm{H}(6 \mathrm{~B}) & 0.9900 \\ \mathrm{C}(7)-\mathrm{C}(25) & 1.524(4) \\ \mathrm{C}(7)-\mathrm{C}(8) & 1.538(4) \\ \mathrm{C}(7)-\mathrm{H}(7) & 1.0000 \\ \mathrm{C}(8)-\mathrm{C}(9) & 1.507(4) \\ \mathrm{C}(8)-\mathrm{H}(8 \mathrm{~A}) & 0.9900 \\ \mathrm{C}(8)-\mathrm{H}(8 \mathrm{~B}) & 1.0000 \\ \mathrm{C}(9)-\mathrm{C}(14) & 0.9800 \\ \mathrm{C}(9)-\mathrm{C}(10) & 0.9800 \\ \mathrm{C}(10)-\mathrm{C}(11) & 1.9900 \\ \mathrm{C}(11)-\mathrm{C}(12) & 1.478(4) \\ \mathrm{C}(12)-\mathrm{C}(13) & 1.499(4) \\ \mathrm{C}(12)-\mathrm{H}(12) & 1.505(4) \\ \mathrm{C}(13)-\mathrm{C}(14) & 1.346(4) \\ \mathrm{C}(15)-\mathrm{C}(16) & 1.454(4) \\ \mathrm{C}(16)-\mathrm{C}(17) & 0.9500 \\ \mathrm{C}(16)-\mathrm{C}(27) & 1.518(4) \\ \mathrm{C}(17)-\mathrm{C}(18) & 1.492(4) \\ \mathrm{C}(17)-\mathrm{H}(17) & 1.345(4) \\ \mathrm{C}(18)-\mathrm{C}(19) & 1.497(4) \\ \mathrm{C}(18)-\mathrm{H}(18) & 1.444(4) \\ \mathrm{C}(19)-\mathrm{C}(20) & 0.9500 \\ \mathrm{C}(19)-\mathrm{H}(19) & 1.334(4) \\ \mathrm{C}(20)-\mathrm{C}(21) & 0.9500 \\ \mathrm{C}(20)-\mathrm{H}(20) & 1.502(4) \\ \mathrm{C}(22)-\mathrm{H}(22 \mathrm{~B}) & \mathrm{H}(21) \\ \end{array}$




\begin{tabular}{|c|c|}
\hline $\mathrm{C}(22)-\mathrm{H}(22 \mathrm{C})$ & 0.9800 \\
\hline $\mathrm{C}(23)-\mathrm{H}(23 \mathrm{~A})$ & 0.9800 \\
\hline $\mathrm{C}(23)-\mathrm{H}(23 \mathrm{~B})$ & 0.9800 \\
\hline $\mathrm{C}(23)-\mathrm{H}(23 \mathrm{C})$ & 0.9800 \\
\hline $\mathrm{C}(24)-\mathrm{H}(24 \mathrm{~A})$ & 0.9800 \\
\hline $\mathrm{C}(24)-\mathrm{H}(24 \mathrm{~B})$ & 0.9800 \\
\hline $\mathrm{C}(24)-\mathrm{H}(24 \mathrm{C})$ & 0.9800 \\
\hline $\mathrm{C}(25)-\mathrm{H}(25 \mathrm{~A})$ & 0.9800 \\
\hline $\mathrm{C}(25)-\mathrm{H}(25 \mathrm{~B})$ & 0.9800 \\
\hline $\mathrm{C}(25)-\mathrm{H}(25 \mathrm{C})$ & 0.9800 \\
\hline $\mathrm{C}(26)-\mathrm{H}(26 \mathrm{~A})$ & 0.9800 \\
\hline $\mathrm{C}(26)-\mathrm{H}(26 \mathrm{~B})$ & 0.9800 \\
\hline $\mathrm{C}(26)-\mathrm{H}(26 \mathrm{C})$ & 0.9800 \\
\hline $\mathrm{C}(27)-\mathrm{H}(27 \mathrm{~A})$ & 0.9800 \\
\hline $\mathrm{C}(27)-\mathrm{H}(27 \mathrm{~B})$ & 0.9800 \\
\hline $\mathrm{C}(27)-\mathrm{H}(27 \mathrm{C})$ & 0.9800 \\
\hline $\mathrm{C}(28)-\mathrm{H}(28 \mathrm{~A})$ & 0.9800 \\
\hline $\mathrm{C}(28)-\mathrm{H}(28 \mathrm{~B})$ & 0.9800 \\
\hline $\mathrm{C}(28)-\mathrm{H}(28 \mathrm{C})$ & 0.9800 \\
\hline $\mathrm{C}(14)-\mathrm{O}(1)-\mathrm{C}(9)$ & $61.41(17)$ \\
\hline $\mathrm{C}(4)-\mathrm{O}(2)-\mathrm{H}(2)$ & $105(2)$ \\
\hline $\mathrm{C}(24)-\mathrm{O}(3)-\mathrm{C}(5)$ & $113.0(2)$ \\
\hline$C(14)-O(5)-C(26)$ & $113.6(2)$ \\
\hline$C(20)-O(8)-C(28)$ & $112.5(2)$ \\
\hline$C(29)-O(9)-C(21)$ & $117.5(2)$ \\
\hline$C(15)-N(1)-C(11)$ & $128.6(2)$ \\
\hline $\mathrm{C}(15)-\mathrm{N}(1)-\mathrm{H}(1)$ & $119(2)$ \\
\hline $\mathrm{C}(11)-\mathrm{N}(1)-\mathrm{H}(1)$ & $112(2)$ \\
\hline $\mathrm{C}(29)-\mathrm{N}(2)-\mathrm{H}(2 \mathrm{~A})$ & $121(2)$ \\
\hline $\mathrm{C}(29)-\mathrm{N}(2)-\mathrm{H}(2 \mathrm{~B})$ & $116(3)$ \\
\hline $\mathrm{H}(2 \mathrm{~A})-\mathrm{N}(2)-\mathrm{H}(2 \mathrm{~B})$ & $123(3)$ \\
\hline$C(2)-C(1)-C(22)$ & $126.0(2)$ \\
\hline$C(2)-C(1)-C(21)$ & $116.5(2)$ \\
\hline$C(22)-C(1)-C(21)$ & $117.4(2)$ \\
\hline$C(1)-C(2)-C(3)$ & $129.4(2)$ \\
\hline
\end{tabular}




$\begin{array}{ll}\mathrm{C}(1)-\mathrm{C}(2)-\mathrm{H}(2 \mathrm{C}) & 115.3 \\ \mathrm{C}(3)-\mathrm{C}(2)-\mathrm{H}(2 \mathrm{C}) & 115.3 \\ \mathrm{C}(2)-\mathrm{C}(3)-\mathrm{C}(23) & 110.5(2) \\ \mathrm{C}(2)-\mathrm{C}(3)-\mathrm{C}(4) & 108.5(2) \\ \mathrm{C}(23)-\mathrm{C}(3)-\mathrm{C}(4) & 113.2(2) \\ \mathrm{C}(2)-\mathrm{C}(3)-\mathrm{H}(3) & 108.2 \\ \mathrm{C}(23)-\mathrm{C}(3)-\mathrm{H}(3) & 108.2 \\ \mathrm{C}(4)-\mathrm{C}(3)-\mathrm{H}(3) & 108.2 \\ \mathrm{O}(2)-\mathrm{C}(4)-\mathrm{C}(5) & 109.29(19) \\ \mathrm{O}(2)-\mathrm{C}(4)-\mathrm{C}(3) & 112.1(2) \\ \mathrm{C}(5)-\mathrm{C}(4)-\mathrm{C}(3) & 114.1(2) \\ \mathrm{O}(2)-\mathrm{C}(4)-\mathrm{H}(4) & 107.0 \\ \mathrm{C}(5)-\mathrm{C}(4)-\mathrm{H}(4) & 107.0 \\ \mathrm{C}(3)-\mathrm{C}(4)-\mathrm{H}(4) & 107.0 \\ \mathrm{O}(3)-\mathrm{C}(5)-\mathrm{C}(6) & 113.8(2) \\ \mathrm{O}(3)-\mathrm{C}(5)-\mathrm{C}(4) & 105.91(19) \\ \mathrm{C}(6)-\mathrm{C}(5)-\mathrm{C}(4) & 113.2(2) \\ \mathrm{O}(3)-\mathrm{C}(5)-\mathrm{H}(5) & 107.9 \\ \mathrm{C}(6)-\mathrm{C}(5)-\mathrm{H}(5) & 107.9 \\ \mathrm{C}(4)-\mathrm{C}(5)-\mathrm{H}(5) & 107.9 \\ \mathrm{C}(7)-\mathrm{C}(6)-\mathrm{C}(5) & 117.0(2) \\ \mathrm{C}(7)-\mathrm{C}(6)-\mathrm{H}(6 \mathrm{~A}) & 108.0 \\ \mathrm{C}(5)-\mathrm{C}(6)-\mathrm{H}(6 \mathrm{~A}) & 108.0 \\ \mathrm{C}(7)-\mathrm{C}(6)-\mathrm{H}(6 \mathrm{~B}) & 108.0 \\ \mathrm{C}(5)-\mathrm{C}(6)-\mathrm{H}(6 \mathrm{~B}) & 108.0 \\ \mathrm{H}(6 \mathrm{~A})-\mathrm{C}(6)-\mathrm{H}(6 \mathrm{~B}) & 107.3 \\ \mathrm{C}(25)-\mathrm{C}(7)-\mathrm{C}(6) & 112.4(2) \\ \mathrm{C}(25)-\mathrm{C}(7)-\mathrm{C}(8) & 107.9(2) \\ \mathrm{C}(6)-\mathrm{C}(7)-\mathrm{C}(8) & 112.0(2) \\ \mathrm{C}(25)-\mathrm{C}(7)-\mathrm{H}(7) & 108.1 \\ \mathrm{C}(6)-\mathrm{C}(7)-\mathrm{H}(7) & 108.1 \\ \mathrm{C}(8)-\mathrm{C}(7)-\mathrm{H}(7) & 108.1 \\ \mathrm{C}(9)-\mathrm{C}(8)-\mathrm{C}(7) & 116.8(2) \\ \mathrm{C}(9)-\mathrm{C}(8)-\mathrm{H}(8 \mathrm{~A}) & 108.1 \\ \mathrm{C}(7)-\mathrm{C}(8)-\mathrm{H}(8 \mathrm{~A}) & 108.1 \\ \mathrm{C}(9)-\mathrm{C}(8)-\mathrm{H}(8 \mathrm{~B}) & 108.1 \\ & \end{array}$




\begin{tabular}{|c|c|}
\hline $\mathrm{C}(7)-\mathrm{C}(8)-\mathrm{H}(8 \mathrm{~B})$ & 108.1 \\
\hline $\mathrm{H}(8 \mathrm{~A})-\mathrm{C}(8)-\mathrm{H}(8 \mathrm{~B})$ & 107.3 \\
\hline $\mathrm{O}(1)-\mathrm{C}(9)-\mathrm{C}(14)$ & $58.00(17)$ \\
\hline$O(1)-C(9)-C(10)$ & $108.7(2)$ \\
\hline$C(14)-C(9)-C(10)$ & $117.2(2)$ \\
\hline $\mathrm{O}(1)-\mathrm{C}(9)-\mathrm{C}(8)$ & $114.2(2)$ \\
\hline$C(14)-C(9)-C(8)$ & $122.9(2)$ \\
\hline$C(10)-C(9)-C(8)$ & $118.1(2)$ \\
\hline $\mathrm{O}(4)-\mathrm{C}(10)-\mathrm{C}(9)$ & $122.2(2)$ \\
\hline $\mathrm{O}(4)-\mathrm{C}(10)-\mathrm{C}(11)$ & $119.2(2)$ \\
\hline$C(9)-C(10)-C(11)$ & $118.5(2)$ \\
\hline$C(12)-C(11)-N(1)$ & $126.6(2)$ \\
\hline$C(12)-C(11)-C(10)$ & $122.4(2)$ \\
\hline$N(1)-C(11)-C(10)$ & $110.9(2)$ \\
\hline$C(11)-C(12)-C(13)$ & $121.9(2)$ \\
\hline $\mathrm{C}(11)-\mathrm{C}(12)-\mathrm{H}(12)$ & 119.0 \\
\hline $\mathrm{C}(13)-\mathrm{C}(12)-\mathrm{H}(12)$ & 119.0 \\
\hline $\mathrm{O}(6)-\mathrm{C}(13)-\mathrm{C}(12)$ & 121.3(3) \\
\hline $\mathrm{O}(6)-\mathrm{C}(13)-\mathrm{C}(14)$ & $120.5(3)$ \\
\hline$C(12)-C(13)-C(14)$ & 118.2(2) \\
\hline $\mathrm{O}(5)-\mathrm{C}(14)-\mathrm{O}(1)$ & $117.6(2)$ \\
\hline $\mathrm{O}(5)-\mathrm{C}(14)-\mathrm{C}(9)$ & $118.5(2)$ \\
\hline $\mathrm{O}(1)-\mathrm{C}(14)-\mathrm{C}(9)$ & $60.59(17)$ \\
\hline $\mathrm{O}(5)-\mathrm{C}(14)-\mathrm{C}(13)$ & $116.6(2)$ \\
\hline $\mathrm{O}(1)-\mathrm{C}(14)-\mathrm{C}(13)$ & $110.6(2)$ \\
\hline$C(9)-C(14)-C(13)$ & $120.0(2)$ \\
\hline $\mathrm{O}(7)-\mathrm{C}(15)-\mathrm{N}(1)$ & $122.0(2)$ \\
\hline $\mathrm{O}(7)-\mathrm{C}(15)-\mathrm{C}(16)$ & $122.5(2)$ \\
\hline$N(1)-C(15)-C(16)$ & $115.5(2)$ \\
\hline$C(17)-C(16)-C(15)$ & $117.9(2)$ \\
\hline$C(17)-C(16)-C(27)$ & $126.1(2)$ \\
\hline$C(15)-C(16)-C(27)$ & $115.8(2)$ \\
\hline$C(16)-C(17)-C(18)$ & 128.3(3) \\
\hline $\mathrm{C}(16)-\mathrm{C}(17)-\mathrm{H}(17)$ & 115.8 \\
\hline $\mathrm{C}(18)-\mathrm{C}(17)-\mathrm{H}(17)$ & 115.8 \\
\hline$C(19)-C(18)-C(17)$ & 123.3(3) \\
\hline
\end{tabular}




\begin{tabular}{|c|c|}
\hline $\mathrm{C}(19)-\mathrm{C}(18)-\mathrm{H}(18)$ & 118.4 \\
\hline $\mathrm{C}(17)-\mathrm{C}(18)-\mathrm{H}(18)$ & 118.4 \\
\hline$C(18)-C(19)-C(20)$ & $126.0(2)$ \\
\hline$C(18)-C(19)-H(19)$ & 117.0 \\
\hline $\mathrm{C}(20)-\mathrm{C}(19)-\mathrm{H}(19)$ & 117.0 \\
\hline $\mathrm{O}(8)-\mathrm{C}(20)-\mathrm{C}(19)$ & $110.7(2)$ \\
\hline $\mathrm{O}(8)-\mathrm{C}(20)-\mathrm{C}(21)$ & $107.7(2)$ \\
\hline$C(19)-C(20)-C(21)$ & $113.3(2)$ \\
\hline $\mathrm{O}(8)-\mathrm{C}(20)-\mathrm{H}(20)$ & 108.3 \\
\hline $\mathrm{C}(19)-\mathrm{C}(20)-\mathrm{H}(20)$ & 108.3 \\
\hline $\mathrm{C}(21)-\mathrm{C}(20)-\mathrm{H}(20)$ & 108.3 \\
\hline $\mathrm{O}(9)-\mathrm{C}(21)-\mathrm{C}(1)$ & $113.5(2)$ \\
\hline $\mathrm{O}(9)-\mathrm{C}(21)-\mathrm{C}(20)$ & $104.8(2)$ \\
\hline$C(1)-C(21)-C(20)$ & $110.2(2)$ \\
\hline $\mathrm{O}(9)-\mathrm{C}(21)-\mathrm{H}(21)$ & 109.4 \\
\hline $\mathrm{C}(1)-\mathrm{C}(21)-\mathrm{H}(21)$ & 109.4 \\
\hline $\mathrm{C}(20)-\mathrm{C}(21)-\mathrm{H}(21)$ & 109.4 \\
\hline$C(1)-C(22)-H(22 A)$ & 109.5 \\
\hline $\mathrm{C}(1)-\mathrm{C}(22)-\mathrm{H}(22 \mathrm{~B})$ & 109.5 \\
\hline$H(22 A)-C(22)-H(22 B)$ & 109.5 \\
\hline$C(1)-C(22)-H(22 C)$ & 109.5 \\
\hline $\mathrm{H}(22 \mathrm{~A})-\mathrm{C}(22)-\mathrm{H}(22 \mathrm{C})$ & 109.5 \\
\hline $\mathrm{H}(22 \mathrm{~B})-\mathrm{C}(22)-\mathrm{H}(22 \mathrm{C})$ & 109.5 \\
\hline$C(3)-C(23)-H(23 A)$ & 109.5 \\
\hline $\mathrm{C}(3)-\mathrm{C}(23)-\mathrm{H}(23 \mathrm{~B})$ & 109.5 \\
\hline $\mathrm{H}(23 \mathrm{~A})-\mathrm{C}(23)-\mathrm{H}(23 \mathrm{~B})$ & 109.5 \\
\hline $\mathrm{C}(3)-\mathrm{C}(23)-\mathrm{H}(23 \mathrm{C})$ & 109.5 \\
\hline$H(23 A)-C(23)-H(23 C)$ & 109.5 \\
\hline $\mathrm{H}(23 \mathrm{~B})-\mathrm{C}(23)-\mathrm{H}(23 \mathrm{C})$ & 109.5 \\
\hline $\mathrm{O}(3)-\mathrm{C}(24)-\mathrm{H}(24 \mathrm{~A})$ & 109.5 \\
\hline $\mathrm{O}(3)-\mathrm{C}(24)-\mathrm{H}(24 \mathrm{~B})$ & 109.5 \\
\hline $\mathrm{H}(24 \mathrm{~A})-\mathrm{C}(24)-\mathrm{H}(24 \mathrm{~B})$ & 109.5 \\
\hline $\mathrm{O}(3)-\mathrm{C}(24)-\mathrm{H}(24 \mathrm{C})$ & 109.5 \\
\hline $\mathrm{H}(24 \mathrm{~A})-\mathrm{C}(24)-\mathrm{H}(24 \mathrm{C})$ & 109.5 \\
\hline $\mathrm{H}(24 \mathrm{~B})-\mathrm{C}(24)-\mathrm{H}(24 \mathrm{C})$ & 109.5 \\
\hline$C(7)-C(25)-H(25 A)$ & 109.5 \\
\hline
\end{tabular}




$\begin{array}{ll}\mathrm{C}(7)-\mathrm{C}(25)-\mathrm{H}(25 \mathrm{~B}) & 109.5 \\ \mathrm{H}(25 \mathrm{~A})-\mathrm{C}(25)-\mathrm{H}(25 \mathrm{~B}) & 109.5 \\ \mathrm{C}(7)-\mathrm{C}(25)-\mathrm{H}(25 \mathrm{C}) & 109.5 \\ \mathrm{H}(25 \mathrm{~A})-\mathrm{C}(25)-\mathrm{H}(25 \mathrm{C}) & 109.5 \\ \mathrm{H}(25 \mathrm{~B})-\mathrm{C}(25)-\mathrm{H}(25 \mathrm{C}) & 109.5 \\ \mathrm{O}(5)-\mathrm{C}(26)-\mathrm{H}(26 \mathrm{~A}) & 109.5 \\ \mathrm{O}(5)-\mathrm{C}(26)-\mathrm{H}(26 \mathrm{~B}) & 109.5 \\ \mathrm{H}(26 \mathrm{~A})-\mathrm{C}(26)-\mathrm{H}(26 \mathrm{~B}) & 109.5 \\ \mathrm{O}(5)-\mathrm{C}(26)-\mathrm{H}(26 \mathrm{C}) & 109.5 \\ \mathrm{H}(26 \mathrm{~A})-\mathrm{C}(26)-\mathrm{H}(26 \mathrm{C}) & 109.5 \\ \mathrm{H}(26 \mathrm{~B})-\mathrm{C}(26)-\mathrm{H}(26 \mathrm{C}) & 109.5 \\ \mathrm{C}(16)-\mathrm{C}(27)-\mathrm{H}(27 \mathrm{~A}) & 109.5 \\ \mathrm{C}(16)-\mathrm{C}(27)-\mathrm{H}(27 \mathrm{~B}) & 109.5 \\ \mathrm{H}(27 \mathrm{~A})-\mathrm{C}(27)-\mathrm{H}(27 \mathrm{~B}) & 109.5 \\ \mathrm{C}(16)-\mathrm{C}(27)-\mathrm{H}(27 \mathrm{C}) & 109.5 \\ \mathrm{H}(27 \mathrm{~A})-\mathrm{C}(27)-\mathrm{H}(27 \mathrm{C}) & 109.5 \\ \mathrm{H}(27 \mathrm{~B})-\mathrm{C}(27)-\mathrm{H}(27 \mathrm{C}) & 109.5 \\ \mathrm{O}(8)-\mathrm{C}(28)-\mathrm{H}(28 \mathrm{~A}) & 109.5 \\ \mathrm{O}(8)-\mathrm{C}(28)-\mathrm{H}(28 \mathrm{~B}) & 109.5 \\ \mathrm{H}(28 \mathrm{~A})-\mathrm{C}(28)-\mathrm{H}(28 \mathrm{~B}) & 109.5 \\ \mathrm{O}(8)-\mathrm{C}(28)-\mathrm{H}(28 \mathrm{C}) & 109.5 \\ \mathrm{H}(28 \mathrm{~A})-\mathrm{C}(28)-\mathrm{H}(28 \mathrm{C}) & 109.5 \\ \mathrm{H}(28 \mathrm{~B})-\mathrm{C}(28)-\mathrm{H}(28 \mathrm{C}) & 109.5 \\ \mathrm{O}(10)-\mathrm{C}(29)-\mathrm{N}(2) & 127.2(3) \\ \mathrm{O}(10)-\mathrm{C}(29)-\mathrm{O}(9) & 122.8(2) \\ \mathrm{N}(2)-\mathrm{C}(29)-\mathrm{O}(9) & 110.1(2) \\ \end{array}$

Symmetry transformations used to generate equivalent atoms:

Table S17. Anisotropic displacement parameters $\left(\AA^{2} \times 10^{3}\right)$ for $\mathbf{D}$. The anisotropic displacement factor exponent takes the form: $-2 \square^{2}\left[h^{2} a^{* 2} U^{11}+\ldots+2 h k a^{*} b^{*} U^{12}\right]$

\begin{tabular}{lllllll}
\hline & $U^{11}$ & $U^{22}$ & $U^{33}$ & $U^{23}$ & $U^{13}$ & $U^{12}$ \\
\hline $\mathrm{O}(1)$ & $45(1)$ & $32(1)$ & $54(1)$ & $\begin{array}{l}-3(1) \\
81\end{array}$ & $6(1)$ & $3(1)$
\end{tabular}




$\begin{array}{lllllll}\mathrm{O}(2) & 50(1) & 28(1) & 36(1) & -1(1) & 4(1) & -1(1) \\ \mathrm{O}(3) & 49(1) & 27(1) & 32(1) & -1(1) & 3(1) & 1(1) \\ \mathrm{O}(4) & 50(1) & 27(1) & 40(1) & -3(1) & 3(1) & -5(1) \\ \mathrm{O}(5) & 64(1) & 29(1) & 48(1) & 3(1) & 14(1) & -2(1) \\ \mathrm{O}(6) & 54(1) & 33(1) & 60(1) & -2(1) & 0(1) & -9(1) \\ \mathrm{O}(7) & 52(1) & 26(1) & 42(1) & -5(1) & -1(1) & 4(1) \\ \mathrm{O}(8) & 38(1) & 24(1) & 49(1) & 4(1) & 2(1) & 4(1) \\ \mathrm{O}(9) & 39(1) & 15(1) & 43(1) & 3(1) & -2(1) & 0(1) \\ \mathrm{O}(10) & 40(1) & 26(1) & 59(1) & 3(1) & -7(1) & -2(1) \\ \mathrm{N}(1) & 50(1) & 21(1) & 37(1) & -3(1) & -1(1) & -1(1) \\ \mathrm{N}(2) & 40(2) & 22(1) & 58(2) & 4(1) & -5(1) & 4(1) \\ \mathrm{C}(1) & 33(1) & 23(1) & 36(1) & -2(1) & -1(1) & 3(1) \\ \mathrm{C}(2) & 43(2) & 25(1) & 37(1) & -1(1) & -4(1) & -1(1) \\ \mathrm{C}(3) & 42(2) & 24(1) & 37(1) & -1(1) & -1(1) & -4(1) \\ \mathrm{C}(4) & 41(2) & 24(1) & 34(1) & 1(1) & 0(1) & -4(1) \\ \mathrm{C}(5) & 40(2) & 24(1) & 34(1) & -1(1) & 0(1) & -2(1) \\ \mathrm{C}(6) & 47(2) & 22(1) & 35(1) & 1(1) & 1(1) & -1(1) \\ \mathrm{C}(7) & 44(2) & 24(1) & 37(1) & -1(1) & 0(1) & 1(1) \\ \mathrm{C}(8) & 45(2) & 30(1) & 38(1) & 1(1) & 0(1) & 4(1) \\ \mathrm{C}(9) & 41(2) & 25(1) & 40(1) & -2(1) & 4(1) & 4(1) \\ \mathrm{C}(10) & 38(1) & 29(1) & 39(1) & -3(1) & 5(1) & 1(1) \\ \mathrm{C}(11) & 41(2) & 26(1) & 36(1) & -2(1) & 3(1) & 1(1) \\ \mathrm{C}(12) & 45(2) & 28(1) & 37(1) & -4(1) & 1(1) & 0(1) \\ \mathrm{C}(13) & 40(2) & 27(1) & 51(2) & -4(1) & 6(1) & -3(1) \\ \mathrm{C}(14) & 44(2) & 27(1) & 44(2) & 0(1) & 9(1) & 0(1) \\ \mathrm{C}(15) & 40(1) & 26(1) & 42(1) & -4(1) & 3(1) & 3(1) \\ \mathrm{C}(16) & 43(2) & 26(1) & 42(1) & -1(1) & 3(1) & 2(1) \\ \mathrm{C}(17) & 49(2) & 28(1) & 40(1) & 1(1) & 5(1) & 2(1) \\ \mathrm{C}(18) & 57(2) & 29(1) & 40(1) & 2(1) & 9(1) & 3(1) \\ \mathrm{C}(19) & 62(2) & 22(1) & 42(1) & 5(1) & 9(1) & 4(1) \\ \mathrm{C}(20) & 48(2) & 16(1) & 40(1) & 2(1) & 7(1) & 2(1) \\ \mathrm{C}(21) & 44(2) & 14(1) & 38(1) & 1(1) & -2(1) & -2(1) \\ \mathrm{C}(22) & 65(2) & 25(1) & 39(1) & -4(1) & 2(1) & -4(1) \\ \mathrm{C}(23) & 43(2) & 31(1) & 48(2) & 3(1) & 3(1) & 0(1) \\ \mathrm{C}(24) & 59(2) & 42(2) & 36(2) & 3(1) & 5(1) & 3(1) \\ \mathrm{C}(25) & 54(2) & 44(2) & 41(2) & -9(1) & -5(1) & 4(1) \\ & & & & 82 & & \\ & & & & & & \end{array}$




$\begin{array}{lllllll}\mathrm{C}(26) & 80(2) & 26(2) & 61(2) & 6(1) & 11(2) & 2(2) \\ \mathrm{C}(27) & 66(2) & 31(2) & 42(2) & 1(1) & 5(1) & 8(1) \\ \mathrm{C}(28) & 45(2) & 40(2) & 75(2) & 9(2) & 1(2) & 14(1) \\ \mathrm{C}(29) & 38(2) & 23(1) & 36(1) & 0(1) & -1(1) & 4(1)\end{array}$

Table S18. Hydrogen coordinates ( x 104) and isotropic displacement parameters $\left(\AA^{2} \times 10^{3}\right)$ for $\mathbf{D}$.

\begin{tabular}{|c|c|c|c|c|}
\hline & $x$ & $y$ & $z$ & $\mathrm{U}(\mathrm{eq})$ \\
\hline $\mathrm{H}(2)$ & $6190(50)$ & $6770(20)$ & $3834(18)$ & $64(11)$ \\
\hline$H(1)$ & $1630(40)$ & $6464(19)$ & $5073(17)$ & $54(10)$ \\
\hline $\mathrm{H}(2 \mathrm{~A})$ & $5140(50)$ & $2145(18)$ & $4996(16)$ & $47(9)$ \\
\hline $\mathrm{H}(2 \mathrm{~B})$ & $7020(50)$ & $2060(20)$ & $5202(19)$ & $66(11)$ \\
\hline $\mathrm{H}(2 \mathrm{C})$ & 6267 & 5305 & 4310 & 42 \\
\hline $\mathrm{H}(3)$ & 6118 & 5134 & 2919 & 41 \\
\hline $\mathrm{H}(4)$ & 4009 & 5867 & 3260 & 39 \\
\hline$H(5)$ & 6432 & 6775 & 2686 & 40 \\
\hline$H(6 A)$ & 4316 & 7489 & 3090 & 42 \\
\hline $\mathrm{H}(6 \mathrm{~B})$ & 4342 & 7511 & 2311 & 42 \\
\hline $\mathrm{H}(7)$ & 2109 & 6578 & 2938 & 42 \\
\hline$H(8 A)$ & 1346 & 8049 & 2710 & 45 \\
\hline $\mathrm{H}(8 \mathrm{~B})$ & 75 & 7467 & 2964 & 45 \\
\hline $\mathrm{H}(12)$ & 3226 & 8141 & 5454 & 44 \\
\hline $\mathrm{H}(17)$ & 2875 & 5661 & 5404 & 47 \\
\hline $\mathrm{H}(18)$ & 2486 & 4654 & 6444 & 51 \\
\hline $\mathrm{H}(19)$ & 3195 & 3815 & 5735 & 50 \\
\hline $\mathrm{H}(20)$ & 3559 & 4935 & 4785 & 42 \\
\hline$H(21)$ & 6107 & 4322 & 5003 & 39 \\
\hline $\mathrm{H}(22 \mathrm{~A})$ & 6275 & 3406 & 3571 & 65 \\
\hline $\mathrm{H}(22 \mathrm{~B})$ & 5905 & 4024 & 3052 & 65 \\
\hline $\mathrm{H}(22 \mathrm{C})$ & 4434 & 3653 & 3439 & 65 \\
\hline $\mathrm{H}(23 \mathrm{~A})$ & 8420 & 6020 & 3600 & 61 \\
\hline
\end{tabular}




\begin{tabular}{lrlll}
$H(23 B)$ & 8301 & 5957 & 2821 & 61 \\
$H(23 C)$ & 8876 & 5286 & 3245 & 61 \\
$H(24 A)$ & 5735 & 6091 & 1182 & 68 \\
$H(24 B)$ & 6956 & 6557 & 1618 & 68 \\
$H(24 C)$ & 5196 & 6865 & 1430 & 68 \\
$H(25 A)$ & 1769 & 7329 & 1728 & 69 \\
$H(25 B)$ & 499 & 6755 & 2004 & 69 \\
$H(25 C)$ & 2288 & 6509 & 1783 & 69 \\
$H(26 A)$ & 2838 & 9990 & 3137 & 83 \\
$H(26 B)$ & 2344 & 9841 & 3882 & 83 \\
$H(26 C)$ & 1069 & 9679 & 3308 & 83 \\
$H(27 A)$ & 2820 & 6316 & 7190 & 69 \\
$H(27 B)$ & 1971 & 5554 & 7101 & 69 \\
$H(27 C)$ & 895 & 6265 & 7063 & 69 \\
$H(28 A)$ & 161 & 4206 & 4129 & 80 \\
$H(28 B)$ & 1123 & 4937 & 4250 & 80 \\
$H(28 C)$ & 435 & 4491 & 4858 & 80 \\
\hline
\end{tabular}

Table S19. Torsion angles $\left[^{\circ}\right]$ for $\mathbf{D}$.

\begin{tabular}{lc}
\hline$C(22)-C(1)-C(2)-C(3)$ & $6.2(5)$ \\
$C(21)-C(1)-C(2)-C(3)$ & $-169.9(3)$ \\
$C(1)-C(2)-C(3)-C(23)$ & $-115.2(3)$ \\
$C(1)-C(2)-C(3)-C(4)$ & $120.1(3)$ \\
$C(2)-C(3)-C(4)-O(2)$ & $52.2(3)$ \\
$C(23)-C(3)-C(4)-O(2)$ & $-70.8(3)$ \\
$C(2)-C(3)-C(4)-C(5)$ & $177.1(2)$ \\
$C(23)-C(3)-C(4)-C(5)$ & $54.0(3)$ \\
$C(24)-O(3)-C(5)-C(6)$ & $75.3(3)$ \\
$C(24)-O(3)-C(5)-C(4)$ & $-159.8(2)$ \\
$O(2)-C(4)-C(5)-O(3)$ & $-177.1(2)$ \\
$C(3)-C(4)-C(5)-O(3)$ & $56.5(3)$ \\
$O(2)-C(4)-C(5)-C(6)$ & $-51.8(3)$ \\
$C(3)-C(4)-C(5)-C(6)$ & $-178.2(2)$
\end{tabular}


$\mathrm{O}(3)-\mathrm{C}(5)-\mathrm{C}(6)-\mathrm{C}(7)$

$\mathrm{C}(4)-\mathrm{C}(5)-\mathrm{C}(6)-\mathrm{C}(7)$

$\mathrm{C}(5)-\mathrm{C}(6)-\mathrm{C}(7)-\mathrm{C}(25)$

$\mathrm{C}(5)-\mathrm{C}(6)-\mathrm{C}(7)-\mathrm{C}(8)$

$C(25)-C(7)-C(8)-C(9)$

$\mathrm{C}(6)-\mathrm{C}(7)-\mathrm{C}(8)-\mathrm{C}(9)$

$C(14)-O(1)-C(9)-C(10)$

$\mathrm{C}(14)-\mathrm{O}(1)-\mathrm{C}(9)-\mathrm{C}(8)$

$\mathrm{C}(7)-\mathrm{C}(8)-\mathrm{C}(9)-\mathrm{O}(1)$

$\mathrm{C}(7)-\mathrm{C}(8)-\mathrm{C}(9)-\mathrm{C}(14)$

$C(7)-C(8)-C(9)-C(10)$

$\mathrm{O}(1)-\mathrm{C}(9)-\mathrm{C}(10)-\mathrm{O}(4)$

$\mathrm{C}(14)-\mathrm{C}(9)-\mathrm{C}(10)-\mathrm{O}(4)$

$\mathrm{C}(8)-\mathrm{C}(9)-\mathrm{C}(10)-\mathrm{O}(4)$

$\mathrm{O}(1)-\mathrm{C}(9)-\mathrm{C}(10)-\mathrm{C}(11)$

$\mathrm{C}(14)-\mathrm{C}(9)-\mathrm{C}(10)-\mathrm{C}(11)$

$\mathrm{C}(8)-\mathrm{C}(9)-\mathrm{C}(10)-\mathrm{C}(11)$

$\mathrm{C}(15)-\mathrm{N}(1)-\mathrm{C}(11)-\mathrm{C}(12)$

$\mathrm{C}(15)-\mathrm{N}(1)-\mathrm{C}(11)-\mathrm{C}(10)$

$\mathrm{O}(4)-\mathrm{C}(10)-\mathrm{C}(11)-\mathrm{C}(12)$

$C(9)-C(10)-C(11)-C(12)$

$\mathrm{O}(4)-\mathrm{C}(10)-\mathrm{C}(11)-\mathrm{N}(1)$

$\mathrm{C}(9)-\mathrm{C}(10)-\mathrm{C}(11)-\mathrm{N}(1)$

$\mathrm{N}(1)-\mathrm{C}(11)-\mathrm{C}(12)-\mathrm{C}(13)$

$C(10)-C(11)-C(12)-C(13)$

$\mathrm{C}(11)-\mathrm{C}(12)-\mathrm{C}(13)-\mathrm{O}(6)$

$C(11)-C(12)-C(13)-C(14)$

$\mathrm{C}(26)-\mathrm{O}(5)-\mathrm{C}(14)-\mathrm{O}(1)$

$\mathrm{C}(26)-\mathrm{O}(5)-\mathrm{C}(14)-\mathrm{C}(9)$

$\mathrm{C}(26)-\mathrm{O}(5)-\mathrm{C}(14)-\mathrm{C}(13)$

$\mathrm{C}(9)-\mathrm{O}(1)-\mathrm{C}(14)-\mathrm{O}(5)$

$\mathrm{C}(9)-\mathrm{O}(1)-\mathrm{C}(14)-\mathrm{C}(13)$

$\mathrm{O}(1)-\mathrm{C}(9)-\mathrm{C}(14)-\mathrm{O}(5)$

$\mathrm{C}(10)-\mathrm{C}(9)-\mathrm{C}(14)-\mathrm{O}(5)$

$\mathrm{C}(8)-\mathrm{C}(9)-\mathrm{C}(14)-\mathrm{O}(5)$

$\mathrm{C}(10)-\mathrm{C}(9)-\mathrm{C}(14)-\mathrm{O}(1)$
59.2(3)

$-61.7(3)$

$-83.7(3)$

154.7(2)

178.3(2)

$-57.5(3)$

110.9(3)

$-114.9(3)$

177.5(2)

111.2(3)

$-52.8(3)$

122.2(3)

$-174.9(3)$

$-10.0(4)$

$-53.9(3)$

9.0(4)

$173.9(2)$

$5.6(5)$

$-177.0(3)$

172.9(3)

$-10.9(4)$

$-4.7(4)$

$171.5(2)$

$177.6(3)$

$0.3(4)$

$-169.2(3)$

11.1(4)

$59.0(3)$

$128.7(3)$

$-76.0(3)$

108.8(3)

$-113.7(2)$

$-107.5(3)$

156.5(2)

$-7.6(4)$

$-96.0(3)$ 


\begin{tabular}{|c|c|}
\hline$C(8)-C(9)-C(14)-O(1)$ & $99.9(3)$ \\
\hline$O(1)-C(9)-C(14)-C(13)$ & $98.1(3)$ \\
\hline$C(10)-C(9)-C(14)-C(13)$ & $2.1(4)$ \\
\hline$C(8)-C(9)-C(14)-C(13)$ & $-162.0(3)$ \\
\hline $\mathrm{O}(6)-\mathrm{C}(13)-\mathrm{C}(14)-\mathrm{O}(5)$ & $13.2(4)$ \\
\hline$C(12)-C(13)-C(14)-O(5)$ & $-167.1(2)$ \\
\hline $\mathrm{O}(6)-\mathrm{C}(13)-\mathrm{C}(14)-\mathrm{O}(1)$ & $-124.8(3)$ \\
\hline$C(12)-C(13)-C(14)-O(1)$ & $54.9(3)$ \\
\hline $\mathrm{O}(6)-\mathrm{C}(13)-\mathrm{C}(14)-\mathrm{C}(9)$ & $168.1(3)$ \\
\hline$C(12)-C(13)-C(14)-C(9)$ & $-12.2(4)$ \\
\hline $\mathrm{C}(11)-\mathrm{N}(1)-\mathrm{C}(15)-\mathrm{O}(7)$ & $15.1(5)$ \\
\hline$C(11)-N(1)-C(15)-C(16)$ & $-165.9(3)$ \\
\hline$O(7)-C(15)-C(16)-C(17)$ & $-155.2(3)$ \\
\hline$N(1)-C(15)-C(16)-C(17)$ & $25.9(4)$ \\
\hline$O(7)-C(15)-C(16)-C(27)$ & $19.5(4)$ \\
\hline$N(1)-C(15)-C(16)-C(27)$ & $-159.4(3)$ \\
\hline$C(15)-C(16)-C(17)-C(18)$ & $179.5(3)$ \\
\hline$C(27)-C(16)-C(17)-C(18)$ & $5.3(6)$ \\
\hline$C(16)-C(17)-C(18)-C(19)$ & $-178.9(3)$ \\
\hline$C(17)-C(18)-C(19)-C(20)$ & $-0.3(5)$ \\
\hline $\mathrm{C}(28)-\mathrm{O}(8)-\mathrm{C}(20)-\mathrm{C}(19)$ & $75.8(3)$ \\
\hline$C(28)-O(8)-C(20)-C(21)$ & $-159.8(2)$ \\
\hline$C(18)-C(19)-C(20)-O(8)$ & $-115.5(3)$ \\
\hline$C(18)-C(19)-C(20)-C(21)$ & $123.4(3)$ \\
\hline$C(29)-O(9)-C(21)-C(1)$ & $91.6(3)$ \\
\hline$C(29)-O(9)-C(21)-C(20)$ & $-148.1(2)$ \\
\hline$C(2)-C(1)-C(21)-O(9)$ & $-168.9(2)$ \\
\hline$C(22)-C(1)-C(21)-O(9)$ & $14.6(3)$ \\
\hline$C(2)-C(1)-C(21)-C(20)$ & 73.9(3) \\
\hline$C(22)-C(1)-C(21)-C(20)$ & $-102.6(3)$ \\
\hline$O(8)-C(20)-C(21)-O(9)$ & $-57.4(2)$ \\
\hline$C(19)-C(20)-C(21)-O(9)$ & $65.4(3)$ \\
\hline$O(8)-C(20)-C(21)-C(1)$ & $65.1(2)$ \\
\hline$C(19)-C(20)-C(21)-C(1)$ & $-172.1(2)$ \\
\hline$C(21)-O(9)-C(29)-O(10)$ & $-4.9(4)$ \\
\hline $\mathrm{C}(21)-\mathrm{O}(9)-\mathrm{C}(29)-\mathrm{N}(2)$ & $175.6(2)$ \\
\hline
\end{tabular}


Symmetry transformations used to generate equivalent atoms:

Table S20. Hydrogen bonds for $\mathbf{D}\left[\AA\right.$ and $\left.^{\circ}\right]$.

\begin{tabular}{lllll}
\hline $\mathrm{D}-\mathrm{H} \ldots \mathrm{A}$ & $\mathrm{d}(\mathrm{D}-\mathrm{H})$ & $\mathrm{d}(\mathrm{H} \ldots \mathrm{A})$ & $\mathrm{d}(\mathrm{D} \ldots \mathrm{A})$ & $<(\mathrm{DHA})$ \\
\hline $\mathrm{O}(2)-\mathrm{H}(2) \ldots \mathrm{O}(7) \# 1$ & $0.96(4)$ & $1.95(4)$ & $2.844(3)$ & $154(3)$ \\
$\mathrm{N}(1)-\mathrm{H}(1) \ldots \mathrm{O}(4)$ & $0.90(4)$ & $2.11(3)$ & $2.606(3)$ & $114(3)$ \\
$\mathrm{N}(1)-\mathrm{H}(1) \ldots \mathrm{O}(6) \# 2$ & $0.90(4)$ & $2.51(4)$ & $3.073(3)$ & $122(3)$ \\
$\mathrm{N}(2)-\mathrm{H}(2 \mathrm{~A}) \ldots \mathrm{O}(10) \# 3$ & $0.93(4)$ & $2.05(4)$ & $2.977(3)$ & $173(3)$ \\
$\mathrm{N}(2)-\mathrm{H}(2 \mathrm{~B}) \ldots \mathrm{O}(8) \# 4$ & $0.89(4)$ & $2.25(4)$ & $2.974(3)$ & $138(3)$ \\
\hline
\end{tabular}

Symmetry transformations used to generate equivalent atoms:

$\# 1 \mathrm{x}+1 / 2,-y+3 / 2,-z+1 \quad \# 2 x-1 / 2,-y+3 / 2,-z+1 \quad \# 3 x-1 / 2,-y+1 / 2,-z+1$

$\# 4 x+1 / 2,-y+1 / 2,-z+1$ 
Refinement details for 17-A:

The methylene alkene group is disordered over three positions. The site occupancies were constrained to be identical, and all chemically identical C-C bonds were restrained to be similar. The thermal parameters were restrained to behave as a rigid, isotropic group with similar values to those in the same disordered group. The program SQUEEZE ${ }^{8}$ was used to compensate for the contribution of disordered solvents contained in voids within the crystal lattice from the diffraction intensities. This procedure was applied to the data file and the submitted model is based on the solvent removed data. Based on the total electron density found in the voids $\left(85 \mathrm{e} / \AA^{3}\right)$, it is likely that 2 dichloromethane solvent molecules are present in the unit cell. See "_platon_squeeze_details" in the .cif for more information.

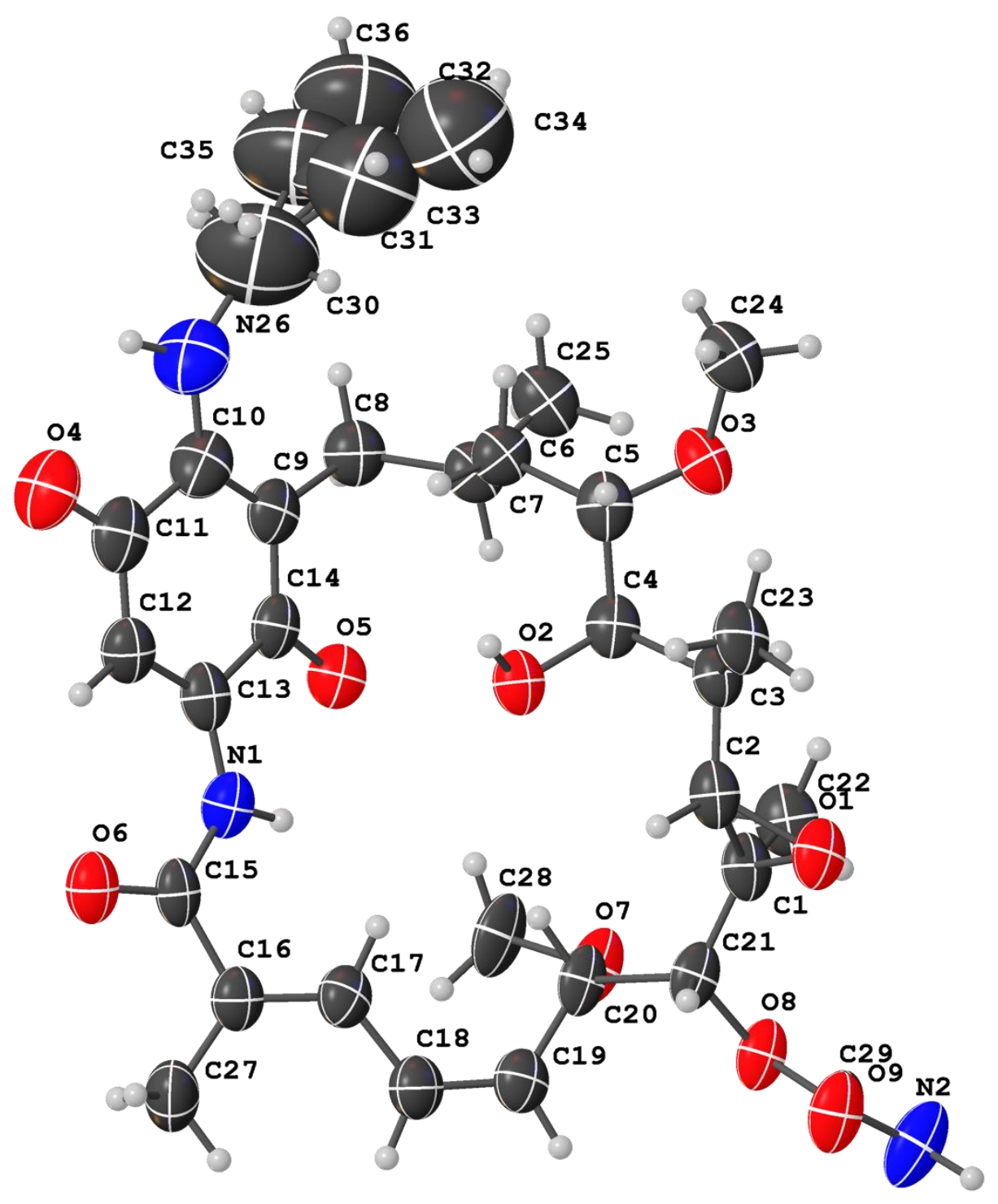

Figure S4. The complete numbering scheme of 17-A with $50 \%$ thermal ellipsoid probability levels. The hydrogen atoms are shown as circles for clarity. 
Table S21. Atomic coordinates $\left(x 10^{4}\right)$ and equivalent isotropic displacement parameters $\left(\AA^{2} x\right.$ $10^{3}$ ) for 17-A. $\mathrm{U}(\mathrm{eq})$ is defined as one third of the trace of the orthogonalized $\mathrm{Uij}$ tensor.

\begin{tabular}{|c|c|c|c|c|}
\hline & $x$ & $y$ & $z$ & $\mathrm{U}(\mathrm{eq})$ \\
\hline $\mathrm{O}(1)$ & $9252(4)$ & $7722(7)$ & $7745(4)$ & $55(2)$ \\
\hline $\mathrm{O}(2)$ & 7270(3) & $5638(7)$ & $5541(4)$ & $50(1)$ \\
\hline $\mathrm{O}(3)$ & $9177(4)$ & $5951(7)$ & $4544(4)$ & $55(2)$ \\
\hline $\mathrm{O}(4)$ & $3850(5)$ & 3193(9) & $2576(5)$ & $77(2)$ \\
\hline $\mathrm{O}(5)$ & $6648(4)$ & $1432(7)$ & $5222(4)$ & $54(1)$ \\
\hline $\mathrm{O}(6)$ & $3787(4)$ & $2870(7)$ & $5752(4)$ & $58(2)$ \\
\hline $\mathrm{O}(7)$ & $8331(4)$ & $2539(6)$ & $8250(4)$ & $55(2)$ \\
\hline $\mathrm{O}(8)$ & $9075(4)$ & $5169(6)$ & $9277(4)$ & $51(2)$ \\
\hline O(9) & $9007(4)$ & 7894(7) & $9724(4)$ & $64(2)$ \\
\hline$N(1)$ & $5238(4)$ & $2434(8)$ & $5681(5)$ & $53(2)$ \\
\hline $\mathrm{N}(2)$ & $10126(6)$ & $6113(9)$ & $10434(5)$ & $73(3)$ \\
\hline$N(26)$ & $5437(7)$ & $2816(13)$ & 2156(6) & $87(3)$ \\
\hline$C(1)$ & $9020(5)$ & $5907(9)$ & $7746(6)$ & $48(2)$ \\
\hline$C(2)$ & $8557(5)$ & $7025(9)$ & $6977(6)$ & $49(2)$ \\
\hline$C(3)$ & $8733(5)$ & 7084(9) & $6097(6)$ & $45(2)$ \\
\hline$C(4)$ & $8186(5)$ & $5708(9)$ & $5466(6)$ & $47(2)$ \\
\hline$C(5)$ & 8224(5) & $5926(10)$ & $4516(6)$ & $53(2)$ \\
\hline$C(6)$ & $7669(6)$ & $4629(10)$ & $3875(6)$ & $51(2)$ \\
\hline$C(7)$ & $7864(5)$ & $2734(9)$ & $4082(6)$ & $49(2)$ \\
\hline$C(8)$ & $7079(6)$ & $1591(11)$ & $3602(7)$ & $58(2)$ \\
\hline $\mathrm{C}(9)$ & $6150(6)$ & $2059(9)$ & $3692(6)$ & $51(2)$ \\
\hline$C(10)$ & $5429(7)$ & $2601(12)$ & $3016(6)$ & $63(2)$ \\
\hline$C(11)$ & $4512(6)$ & $2941(11)$ & $3210(7)$ & $63(2)$ \\
\hline$C(12)$ & $4430(5)$ & $2916(10)$ & $4107(6)$ & $53(2)$ \\
\hline$C(13)$ & $5172(5)$ & $2487(9)$ & $4782(6)$ & $49(2)$ \\
\hline$C(14)$ & $6043(5)$ & 1929(9) & $4571(6)$ & $47(2)$ \\
\hline$C(15)$ & $4582(5)$ & $2771(9)$ & $6118(6)$ & $46(2)$ \\
\hline$C(16)$ & $4921(5)$ & $2991(10)$ & $7113(6)$ & $50(2)$ \\
\hline$C(17)$ & $5785(6)$ & $3457(10)$ & $7453(6)$ & $52(2)$ \\
\hline$C(18)$ & $6248(6)$ & $3709(11)$ & $8376(6)$ & $56(2)$ \\
\hline$C(19)$ & $7141(6)$ & $3998(10)$ & $8690(6)$ & $53(2)$ \\
\hline
\end{tabular}




$\begin{array}{lllll}\mathrm{C}(20) & 7813(5) & 4076(8) & 8158(6) & 47(2) \\ \mathrm{C}(21) & 8479(5) & 5563(8) & 8403(5) & 47(2) \\ \mathrm{C}(22) & 9742(6) & 4697(11) & 7663(7) & 62(2) \\ \mathrm{C}(23) & 8551(6) & 8917(10) & 5722(7) & 61(2) \\ \mathrm{C}(24) & 9379(7) & 6728(12) & 3791(7) & 65(2) \\ \mathrm{C}(25) & 8743(6) & 2182(11) & 3868(7) & 66(2) \\ \mathrm{C}(27) & 4260(6) & 2719(15) & 7624(7) & 70(3) \\ \mathrm{C}(28) & 7842(7) & 1112(10) & 7794(7) & 68(3) \\ \mathrm{C}(29) & 9381(6) & 6509(10) & 9819(6) & 55(2) \\ \mathrm{C}(30) & 6142(17) & 2950(40) & 1621(17) & 194(9) \\ \mathrm{C}(31) & 7000(50) & 3810(150) & 1380(80) & 206(19) \\ \mathrm{C}(32) & 7840(30) & 3420(80) & 1350(40) & 115(15) \\ \mathrm{C}(33) & 7060(30) & 3070(90) & 1330(40) & 132(15) \\ \mathrm{C}(34) & 7750(40) & 4470(150) & 1310(70) & 210(30) \\ \mathrm{C}(35) & 6850(60) & 2230(140) & 1130(70) & 200(20) \\ \mathrm{C}(36) & 7560(40) & 1640(100) & 980(50) & 190(30)\end{array}$

Table 22. Bond lengths $[\AA]$ and angles $\left[^{\circ}\right]$ for 17-A.

\begin{tabular}{ll}
\hline $\mathrm{O}(1)-\mathrm{C}(1)$ & $1.470(9)$ \\
$\mathrm{O}(1)-\mathrm{C}(2)$ & $1.479(9)$ \\
$\mathrm{O}(2)-\mathrm{C}(4)$ & $1.432(10)$ \\
$\mathrm{O}(2)-\mathrm{H}(2)$ & 0.8400 \\
$\mathrm{O}(3)-\mathrm{C}(24)$ & $1.421(11)$ \\
$\mathrm{O}(3)-\mathrm{C}(5)$ & $1.443(10)$ \\
$\mathrm{O}(4)-\mathrm{C}(11)$ & $1.228(11)$ \\
$\mathrm{O}(5)-\mathrm{C}(14)$ & $1.241(9)$ \\
$\mathrm{O}(6)-\mathrm{C}(15)$ & $1.200(8)$ \\
$\mathrm{O}(7)-\mathrm{C}(28)$ & $1.429(9)$ \\
$\mathrm{O}(7)-\mathrm{C}(20)$ & $1.430(8)$ \\
$\mathrm{O}(8)-\mathrm{C}(29)$ & $1.353(9)$ \\
$\mathrm{O}(8)-\mathrm{C}(21)$ & $1.456(9)$ \\
$\mathrm{O}(9)-\mathrm{C}(29)$ & $1.220(10)$ \\
$\mathrm{N}(1)-\mathrm{C}(15)$ & $1.371(11)$
\end{tabular}




$\begin{array}{ll}\mathrm{N}(1)-\mathrm{C}(13) & 1.373(11) \\ \mathrm{N}(1)-\mathrm{H}(1) & 0.8800 \\ \mathrm{~N}(2)-\mathrm{C}(29) & 1.317(11) \\ \mathrm{N}(2)-\mathrm{H}(2 \mathrm{~A}) & 0.8800 \\ \mathrm{~N}(2)-\mathrm{H}(2 \mathrm{~B}) & 0.8800 \\ \mathrm{~N}(26)-\mathrm{C}(10) & 1.349(13) \\ \mathrm{N}(26)-\mathrm{C}(30) & 1.52(2) \\ \mathrm{N}(26)-\mathrm{H}(26) & 0.8800 \\ \mathrm{C}(1)-\mathrm{C}(22) & 1.485(12) \\ \mathrm{C}(1)-\mathrm{C}(21) & 1.492(13) \\ \mathrm{C}(1)-\mathrm{C}(2) & 1.501(11) \\ \mathrm{C}(2)-\mathrm{C}(3) & 1.459(12) \\ \mathrm{C}(2)-\mathrm{H}(2 \mathrm{C}) & 1.0000 \\ \mathrm{C}(3)-\mathrm{C}(4) & 1.550(11) \\ \mathrm{C}(3)-\mathrm{C}(23) & 1.552(11) \\ \mathrm{C}(3)-\mathrm{H}(3) & 1.0000 \\ \mathrm{C}(4)-\mathrm{C}(5) & 1.498(13) \\ \mathrm{C}(4)-\mathrm{H}(4) & 1.0000 \\ \mathrm{C}(5)-\mathrm{C}(6) & 1.519(12) \\ \mathrm{C}(5)-\mathrm{H}(5) & 1.0000 \\ \mathrm{C}(6)-\mathrm{C}(7) & 1.536(10) \\ \mathrm{C}(6)-\mathrm{H}(6 \mathrm{~A}) & 0.9900 \\ \mathrm{C}(6)-\mathrm{H}(6 \mathrm{~B}) & 0.9900 \\ \mathrm{C}(7)-\mathrm{C}(25) & 1.525(12) \\ \mathrm{C}(7)-\mathrm{C}(8) & 1.527(12) \\ \mathrm{C}(7)-\mathrm{H}(7) & 1.0000 \\ \mathrm{C}(8)-\mathrm{C}(9) & 1.504(13) \\ \mathrm{C}(8)-\mathrm{H}(8 \mathrm{~A}) & 0.9900 \\ \mathrm{C}(8)-\mathrm{H}(8 \mathrm{~B}) & 0.9900 \\ \mathrm{C}(9)-\mathrm{C}(10) & 1.376(12) \\ \mathrm{C}(9)-\mathrm{C}(14) & 1.418(12) \\ \mathrm{C}(10)-\mathrm{C}(11) & 1.527(14) \\ \mathrm{C}(11)-\mathrm{C}(12) & 1.430(13) \\ \mathrm{C}(12)-\mathrm{C}(13) & 1.370(11) \\ \mathrm{C}(12)-\mathrm{H}(12) & 0.9500 \\ \mathrm{C}(13)-\mathrm{C}(14) & 1.512(12) \\ & \end{array}$




\begin{tabular}{|c|c|}
\hline$C(15)-C(16)$ & $1.506(12)$ \\
\hline$C(16)-C(17)$ & $1.336(11)$ \\
\hline$C(16)-C(27)$ & $1.451(13)$ \\
\hline$C(17)-C(18)$ & $1.436(13)$ \\
\hline $\mathrm{C}(17)-\mathrm{H}(17)$ & 0.9500 \\
\hline$C(18)-C(19)$ & $1.340(12)$ \\
\hline $\mathrm{C}(18)-\mathrm{H}(18)$ & 0.9500 \\
\hline$C(19)-C(20)$ & $1.475(13)$ \\
\hline $\mathrm{C}(19)-\mathrm{H}(19)$ & 0.9500 \\
\hline$C(20)-C(21)$ & $1.530(10)$ \\
\hline $\mathrm{C}(20)-\mathrm{H}(20)$ & 1.0000 \\
\hline $\mathrm{C}(21)-\mathrm{H}(21)$ & 1.0000 \\
\hline $\mathrm{C}(22)-\mathrm{H}(22 \mathrm{~A})$ & 0.9800 \\
\hline $\mathrm{C}(22)-\mathrm{H}(22 \mathrm{~B})$ & 0.9800 \\
\hline $\mathrm{C}(22)-\mathrm{H}(22 \mathrm{C})$ & 0.9800 \\
\hline $\mathrm{C}(23)-\mathrm{H}(23 \mathrm{~A})$ & 0.9800 \\
\hline $\mathrm{C}(23)-\mathrm{H}(23 \mathrm{~B})$ & 0.9800 \\
\hline $\mathrm{C}(23)-\mathrm{H}(23 \mathrm{C})$ & 0.9800 \\
\hline$C(24)-H(24 A)$ & 0.9800 \\
\hline $\mathrm{C}(24)-\mathrm{H}(24 \mathrm{~B})$ & 0.9800 \\
\hline$C(24)-H(24 C)$ & 0.9800 \\
\hline $\mathrm{C}(25)-\mathrm{H}(25 \mathrm{~A})$ & 0.9800 \\
\hline $\mathrm{C}(25)-\mathrm{H}(25 \mathrm{~B})$ & 0.9800 \\
\hline $\mathrm{C}(25)-\mathrm{H}(25 \mathrm{C})$ & 0.9800 \\
\hline $\mathrm{C}(27)-\mathrm{H}(27 \mathrm{~A})$ & 0.9800 \\
\hline $\mathrm{C}(27)-\mathrm{H}(27 \mathrm{~B})$ & 0.9800 \\
\hline $\mathrm{C}(27)-\mathrm{H}(27 \mathrm{C})$ & 0.9800 \\
\hline $\mathrm{C}(28)-\mathrm{H}(28 \mathrm{~A})$ & 0.9800 \\
\hline $\mathrm{C}(28)-\mathrm{H}(28 \mathrm{~B})$ & 0.9800 \\
\hline $\mathrm{C}(28)-\mathrm{H}(28 \mathrm{C})$ & 0.9800 \\
\hline$C(30)-C(33)$ & $1.58(2)$ \\
\hline$C(30)-C(35)$ & $1.58(2)$ \\
\hline$C(30)-C(31)$ & $1.59(2)$ \\
\hline$C(30)-H(30 A)$ & 0.9900 \\
\hline $\mathrm{C}(30)-\mathrm{H}(30 \mathrm{~B})$ & 0.9900 \\
\hline $\mathrm{C}(30)-\mathrm{H}(30 \mathrm{E})$ & 0.9900 \\
\hline
\end{tabular}




\begin{tabular}{|c|c|}
\hline $\mathrm{C}(30)-\mathrm{H}(30 \mathrm{~F})$ & 0.9900 \\
\hline $\mathrm{C}(30)-\mathrm{H}(30 \mathrm{C})$ & 0.9900 \\
\hline $\mathrm{C}(30)-\mathrm{H}(30 \mathrm{D})$ & 0.9900 \\
\hline$C(31)-C(32)$ & $1.33(9)$ \\
\hline $\mathrm{C}(31)-\mathrm{H}(31)$ & 0.9500 \\
\hline $\mathrm{C}(32)-\mathrm{H}(32 \mathrm{~A})$ & 0.9500 \\
\hline $\mathrm{C}(32)-\mathrm{H}(32 \mathrm{~B})$ & 0.9500 \\
\hline$C(33)-C(34)$ & $1.52(8)$ \\
\hline $\mathrm{C}(33)-\mathrm{H}(33)$ & 0.9500 \\
\hline $\mathrm{C}(34)-\mathrm{H}(34 \mathrm{~A})$ & 0.9500 \\
\hline $\mathrm{C}(34)-\mathrm{H}(34 \mathrm{~B})$ & 0.9500 \\
\hline$C(35)-C(36)$ & $1.26(3)$ \\
\hline $\mathrm{C}(35)-\mathrm{H}(35)$ & 0.9500 \\
\hline$C(36)-H(36 A)$ & 0.9500 \\
\hline $\mathrm{C}(36)-\mathrm{H}(36 \mathrm{~B})$ & 0.9500 \\
\hline$C(1)-O(1)-C(2)$ & $61.2(5)$ \\
\hline $\mathrm{C}(4)-\mathrm{O}(2)-\mathrm{H}(2)$ & 109.5 \\
\hline $\mathrm{C}(24)-\mathrm{O}(3)-\mathrm{C}(5)$ & $114.5(6)$ \\
\hline $\mathrm{C}(28)-\mathrm{O}(7)-\mathrm{C}(20)$ & $114.2(6)$ \\
\hline $\mathrm{C}(29)-\mathrm{O}(8)-\mathrm{C}(21)$ & $116.4(6)$ \\
\hline$C(15)-N(1)-C(13)$ & $128.8(7)$ \\
\hline $\mathrm{C}(15)-\mathrm{N}(1)-\mathrm{H}(1)$ & 115.6 \\
\hline $\mathrm{C}(13)-\mathrm{N}(1)-\mathrm{H}(1)$ & 115.6 \\
\hline $\mathrm{C}(29)-\mathrm{N}(2)-\mathrm{H}(2 \mathrm{~A})$ & 120.0 \\
\hline $\mathrm{C}(29)-\mathrm{N}(2)-\mathrm{H}(2 \mathrm{~B})$ & 120.0 \\
\hline $\mathrm{H}(2 \mathrm{~A})-\mathrm{N}(2)-\mathrm{H}(2 \mathrm{~B})$ & 120.0 \\
\hline$C(10)-N(26)-C(30)$ & $137.5(13)$ \\
\hline $\mathrm{C}(10)-\mathrm{N}(26)-\mathrm{H}(26)$ & 111.2 \\
\hline $\mathrm{C}(30)-\mathrm{N}(26)-\mathrm{H}(26)$ & 111.2 \\
\hline $\mathrm{O}(1)-\mathrm{C}(1)-\mathrm{C}(22)$ & $115.9(7)$ \\
\hline $\mathrm{O}(1)-\mathrm{C}(1)-\mathrm{C}(21)$ & $110.6(6)$ \\
\hline$C(22)-C(1)-C(21)$ & $119.7(7)$ \\
\hline $\mathrm{O}(1)-\mathrm{C}(1)-\mathrm{C}(2)$ & $59.7(5)$ \\
\hline$C(22)-C(1)-C(2)$ & $120.8(8)$ \\
\hline$C(21)-C(1)-C(2)$ & $114.8(7)$ \\
\hline
\end{tabular}




$\begin{array}{ll}\mathrm{C}(3)-\mathrm{C}(2)-\mathrm{O}(1) & 118.3(6) \\ \mathrm{C}(3)-\mathrm{C}(2)-\mathrm{C}(1) & 126.8(7) \\ \mathrm{O}(1)-\mathrm{C}(2)-\mathrm{C}(1) & 59.1(5) \\ \mathrm{C}(3)-\mathrm{C}(2)-\mathrm{H}(2 \mathrm{C}) & 113.7 \\ \mathrm{O}(1)-\mathrm{C}(2)-\mathrm{H}(2 \mathrm{C}) & 113.7 \\ \mathrm{C}(1)-\mathrm{C}(2)-\mathrm{H}(2 \mathrm{C}) & 113.7 \\ \mathrm{C}(2)-\mathrm{C}(3)-\mathrm{C}(4) & 111.7(6) \\ \mathrm{C}(2)-\mathrm{C}(3)-\mathrm{C}(23) & 108.8(7) \\ \mathrm{C}(4)-\mathrm{C}(3)-\mathrm{C}(23) & 113.4(7) \\ \mathrm{C}(2)-\mathrm{C}(3)-\mathrm{H}(3) & 107.6 \\ \mathrm{C}(4)-\mathrm{C}(3)-\mathrm{H}(3) & 107.6 \\ \mathrm{C}(23)-\mathrm{C}(3)-\mathrm{H}(3) & 107.6 \\ \mathrm{O}(2)-\mathrm{C}(4)-\mathrm{C}(5) & 111.8(6) \\ \mathrm{O}(2)-\mathrm{C}(4)-\mathrm{C}(3) & 110.6(6) \\ \mathrm{C}(5)-\mathrm{C}(4)-\mathrm{C}(3) & 113.0(7) \\ \mathrm{O}(2)-\mathrm{C}(4)-\mathrm{H}(4) & 107.0 \\ \mathrm{C}(5)-\mathrm{C}(4)-\mathrm{H}(4) & 107.0 \\ \mathrm{C}(3)-\mathrm{C}(4)-\mathrm{H}(4) & 107.0 \\ \mathrm{O}(3)-\mathrm{C}(5)-\mathrm{C}(4) & 105.5(6) \\ \mathrm{O}(3)-\mathrm{C}(5)-\mathrm{C}(6) & 114.4(7) \\ \mathrm{C}(4)-\mathrm{C}(5)-\mathrm{C}(6) & 114.2(7) \\ \mathrm{O}(3)-\mathrm{C}(5)-\mathrm{H}(5) & 107.4 \\ \mathrm{C}(4)-\mathrm{C}(5)-\mathrm{H}(5) & 107.4 \\ \mathrm{C}(6)-\mathrm{C}(5)-\mathrm{H}(5) & 107.4 \\ \mathrm{C}(5)-\mathrm{C}(6)-\mathrm{C}(7) & 118.0(7) \\ \mathrm{C}(5)-\mathrm{C}(6)-\mathrm{H}(6 \mathrm{~A}) & 107.8 \\ \mathrm{C}(7)-\mathrm{C}(6)-\mathrm{H}(6 \mathrm{~A}) & 107.8 \\ \mathrm{C}(5)-\mathrm{C}(6)-\mathrm{H}(6 \mathrm{~B}) & 107.8 \\ \mathrm{C}(7)-\mathrm{C}(6)-\mathrm{H}(6 \mathrm{~B}) & 107.8 \\ \mathrm{H}(6 \mathrm{~A})-\mathrm{C}(6)-\mathrm{H}(6 \mathrm{~B}) & 107.1 \\ \mathrm{C}(25)-\mathrm{C}(7)-\mathrm{C}(8) & 110.4(7) \\ \mathrm{C}(25)-\mathrm{C}(7)-\mathrm{C}(6) & 111.3(7) \\ \mathrm{C}(8)-\mathrm{C}(7)-\mathrm{C}(6) & 112.6(7) \\ \mathrm{C}(25)-\mathrm{C}(7)-\mathrm{H}(7) & 107.5 \\ \mathrm{C}(8)-\mathrm{C}(7)-\mathrm{H}(7) & 107.5 \\ \mathrm{C}(6)-\mathrm{C}(7)-\mathrm{H}(7) & 107.5 \\ & \end{array}$




$\begin{array}{ll}\mathrm{C}(9)-\mathrm{C}(8)-\mathrm{C}(7) & 116.6(7) \\ \mathrm{C}(9)-\mathrm{C}(8)-\mathrm{H}(8 \mathrm{~A}) & 108.1 \\ \mathrm{C}(7)-\mathrm{C}(8)-\mathrm{H}(8 \mathrm{~A}) & 108.1 \\ \mathrm{C}(9)-\mathrm{C}(8)-\mathrm{H}(8 \mathrm{~B}) & 108.1 \\ \mathrm{C}(7)-\mathrm{C}(8)-\mathrm{H}(8 \mathrm{~B}) & 108.1 \\ \mathrm{H}(8 \mathrm{~A})-\mathrm{C}(8)-\mathrm{H}(8 \mathrm{~B}) & 107.3 \\ \mathrm{C}(10)-\mathrm{C}(9)-\mathrm{C}(14) & 119.1(8) \\ \mathrm{C}(10)-\mathrm{C}(9)-\mathrm{C}(8) & 126.2(9) \\ \mathrm{C}(14)-\mathrm{C}(9)-\mathrm{C}(8) & 114.8(7) \\ \mathrm{N}(26)-\mathrm{C}(10)-\mathrm{C}(9) & 125.6(10) \\ \mathrm{N}(26)-\mathrm{C}(10)-\mathrm{C}(11) & 114.6(8) \\ \mathrm{C}(9)-\mathrm{C}(10)-\mathrm{C}(11) & 119.7(9) \\ \mathrm{O}(4)-\mathrm{C}(11)-\mathrm{C}(12) & 121.3(9) \\ \mathrm{O}(4)-\mathrm{C}(11)-\mathrm{C}(10) & 118.3(9) \\ \mathrm{C}(12)-\mathrm{C}(11)-\mathrm{C}(10) & 120.3(8) \\ \mathrm{C}(13)-\mathrm{C}(12)-\mathrm{C}(11) & 119.0(8) \\ \mathrm{C}(13)-\mathrm{C}(12)-\mathrm{H}(12) & 120.5 \\ \mathrm{C}(11)-\mathrm{C}(12)-\mathrm{H}(12) & 120.5 \\ \mathrm{C}(12)-\mathrm{C}(13)-\mathrm{N}(1) & 127.8(8) \\ \mathrm{C}(12)-\mathrm{C}(13)-\mathrm{C}(14) & 120.2(8) \\ \mathrm{N}(1)-\mathrm{C}(13)-\mathrm{C}(14) & 112.0(7) \\ \mathrm{O}(5)-\mathrm{C}(14)-\mathrm{C}(9) & 123.9(8) \\ \mathrm{O}(5)-\mathrm{C}(14)-\mathrm{C}(13) & 115.1(8) \\ \mathrm{C}(9)-\mathrm{C}(14)-\mathrm{C}(13) & 121.0(7) \\ \mathrm{O}(6)-\mathrm{C}(15)-\mathrm{N}(1) & 123.7(8) \\ \mathrm{O}(6)-\mathrm{C}(15)-\mathrm{C}(16) & 120.9(7) \\ \mathrm{N}(1)-\mathrm{C}(15)-\mathrm{C}(16) & 115.4(6) \\ \mathrm{C}(17)-\mathrm{C}(16)-\mathrm{C}(27) & 125.3(8) \\ \mathrm{C}(17)-\mathrm{C}(16)-\mathrm{C}(15) & 118.5(8) \\ \mathrm{C}(27)-\mathrm{C}(16)-\mathrm{C}(15) & 116.2(7) \\ \mathrm{C}(16)-\mathrm{C}(17)-\mathrm{C}(18) & 127.6(9) \\ \mathrm{C}(16)-\mathrm{C}(17)-\mathrm{H}(17) & 116.2 \\ \mathrm{C}(18)-\mathrm{C}(17)-\mathrm{H}(17) & 116.2 \\ \mathrm{C}(19)-\mathrm{C}(18)-\mathrm{C}(17) & 125.1(9) \\ \mathrm{C}(19)-\mathrm{C}(18)-\mathrm{H}(18) & 117.4 \\ \mathrm{C}(17)-\mathrm{C}(18)-\mathrm{H}(18) & 117.4 \\ & \end{array}$




$\begin{array}{ll}\mathrm{C}(18)-\mathrm{C}(19)-\mathrm{C}(20) & 126.2(9) \\ \mathrm{C}(18)-\mathrm{C}(19)-\mathrm{H}(19) & 116.9 \\ \mathrm{C}(20)-\mathrm{C}(19)-\mathrm{H}(19) & 116.9 \\ \mathrm{O}(7)-\mathrm{C}(20)-\mathrm{C}(19) & 110.8(6) \\ \mathrm{O}(7)-\mathrm{C}(20)-\mathrm{C}(21) & 107.9(6) \\ \mathrm{C}(19)-\mathrm{C}(20)-\mathrm{C}(21) & 113.9(7) \\ \mathrm{O}(7)-\mathrm{C}(20)-\mathrm{H}(20) & 108.0 \\ \mathrm{C}(19)-\mathrm{C}(20)-\mathrm{H}(20) & 108.0 \\ \mathrm{C}(21)-\mathrm{C}(20)-\mathrm{H}(20) & 108.0 \\ \mathrm{O}(8)-\mathrm{C}(21)-\mathrm{C}(1) & 110.7(6) \\ \mathrm{O}(8)-\mathrm{C}(21)-\mathrm{C}(20) & 106.4(6) \\ \mathrm{C}(1)-\mathrm{C}(21)-\mathrm{C}(20) & 114.6(7) \\ \mathrm{O}(8)-\mathrm{C}(21)-\mathrm{H}(21) & 108.3 \\ \mathrm{C}(1)-\mathrm{C}(21)-\mathrm{H}(21) & 108.3 \\ \mathrm{C}(20)-\mathrm{C}(21)-\mathrm{H}(21) & 108.3 \\ \mathrm{C}(1)-\mathrm{C}(22)-\mathrm{H}(22 \mathrm{~A}) & 109.5 \\ \mathrm{C}(1)-\mathrm{C}(22)-\mathrm{H}(22 \mathrm{~B}) & 109.5 \\ \mathrm{H}(22 \mathrm{~A})-\mathrm{C}(22)-\mathrm{H}(22 \mathrm{~B}) & 109.5 \\ \mathrm{C}(1)-\mathrm{C}(22)-\mathrm{H}(22 \mathrm{C}) & 109.5 \\ \mathrm{H}(22 \mathrm{~A})-\mathrm{C}(22)-\mathrm{H}(22 \mathrm{C}) & 109.5 \\ \mathrm{H}(22 \mathrm{~B})-\mathrm{C}(22)-\mathrm{H}(22 \mathrm{C}) & 109.5 \\ \mathrm{C}(3)-\mathrm{C}(23)-\mathrm{H}(23 \mathrm{~A}) & 109.5 \\ \mathrm{C}(3)-\mathrm{C}(23)-\mathrm{H}(23 \mathrm{~B}) & 109.5 \\ \mathrm{H}(23 \mathrm{~A})-\mathrm{C}(23)-\mathrm{H}(23 \mathrm{~B}) & 109.5 \\ \mathrm{C}(3)-\mathrm{C}(23)-\mathrm{H}(23 \mathrm{C}) & 109.5 \\ \mathrm{H}(23 \mathrm{~A})-\mathrm{C}(23)-\mathrm{H}(23 \mathrm{C}) & 109.5 \\ \mathrm{H}(23 \mathrm{~B})-\mathrm{C}(23)-\mathrm{H}(23 \mathrm{C}) & 109.5 \\ \mathrm{O}(3)-\mathrm{C}(24)-\mathrm{H}(24 \mathrm{~A}) & 109.5 \\ \mathrm{O}(3)-\mathrm{C}(24)-\mathrm{H}(24 \mathrm{~B}) & 109.5 \\ \mathrm{H}(24 \mathrm{~A})-\mathrm{C}(24)-\mathrm{H}(24 \mathrm{~B}) & 109.5 \\ \mathrm{O}(3)-\mathrm{C}(24)-\mathrm{H}(24 \mathrm{C}) & 109.5 \\ \mathrm{H}(24 \mathrm{~A})-\mathrm{C}(24)-\mathrm{H}(24 \mathrm{C}) & 109.5 \\ \mathrm{H}(24 \mathrm{~B})-\mathrm{C}(24)-\mathrm{H}(24 \mathrm{C}) & 109.5 \\ \mathrm{C}(7)-\mathrm{C}(25)-\mathrm{H}(25 \mathrm{~A}) & 109.5 \\ \mathrm{C}(7)-\mathrm{C}(25)-\mathrm{H}(25 \mathrm{~B}) & 109.5 \\ \mathrm{H}(25 \mathrm{~A})-\mathrm{C}(25)-\mathrm{H}(25 \mathrm{~B}) & 109.5 \\ & \\ & \end{array}$




\begin{tabular}{|c|c|}
\hline $\mathrm{C}(7)-\mathrm{C}(25)-\mathrm{H}(25 \mathrm{C})$ & 109.5 \\
\hline$H(25 A)-C(25)-H(25 C)$ & 109.5 \\
\hline$H(25 B)-C(25)-H(25 C)$ & 109.5 \\
\hline$C(16)-C(27)-H(27 A)$ & 109.5 \\
\hline $\mathrm{C}(16)-\mathrm{C}(27)-\mathrm{H}(27 \mathrm{~B})$ & 109.5 \\
\hline$H(27 A)-C(27)-H(27 B)$ & 109.5 \\
\hline $\mathrm{C}(16)-\mathrm{C}(27)-\mathrm{H}(27 \mathrm{C})$ & 109.5 \\
\hline $\mathrm{H}(27 \mathrm{~A})-\mathrm{C}(27)-\mathrm{H}(27 \mathrm{C})$ & 109.5 \\
\hline $\mathrm{H}(27 \mathrm{~B})-\mathrm{C}(27)-\mathrm{H}(27 \mathrm{C})$ & 109.5 \\
\hline $\mathrm{O}(7)-\mathrm{C}(28)-\mathrm{H}(28 \mathrm{~A})$ & 109.5 \\
\hline $\mathrm{O}(7)-\mathrm{C}(28)-\mathrm{H}(28 \mathrm{~B})$ & 109.5 \\
\hline $\mathrm{H}(28 \mathrm{~A})-\mathrm{C}(28)-\mathrm{H}(28 \mathrm{~B})$ & 109.5 \\
\hline $\mathrm{O}(7)-\mathrm{C}(28)-\mathrm{H}(28 \mathrm{C})$ & 109.5 \\
\hline $\mathrm{H}(28 \mathrm{~A})-\mathrm{C}(28)-\mathrm{H}(28 \mathrm{C})$ & 109.5 \\
\hline $\mathrm{H}(28 \mathrm{~B})-\mathrm{C}(28)-\mathrm{H}(28 \mathrm{C})$ & 109.5 \\
\hline $\mathrm{O}(9)-\mathrm{C}(29)-\mathrm{N}(2)$ & $125.9(7)$ \\
\hline $\mathrm{O}(9)-\mathrm{C}(29)-\mathrm{O}(8)$ & $123.0(7)$ \\
\hline $\mathrm{N}(2)-\mathrm{C}(29)-\mathrm{O}(8)$ & 111.1(7) \\
\hline$N(26)-C(30)-C(33)$ & $164(3)$ \\
\hline$N(26)-C(30)-C(35)$ & $155(5)$ \\
\hline$N(26)-C(30)-C(31)$ & $153(5)$ \\
\hline$N(26)-C(30)-H(30 A)$ & 94.9 \\
\hline$C(33)-C(30)-H(30 A)$ & 94.9 \\
\hline $\mathrm{N}(26)-\mathrm{C}(30)-\mathrm{H}(30 \mathrm{~B})$ & 94.9 \\
\hline $\mathrm{C}(33)-\mathrm{C}(30)-\mathrm{H}(30 \mathrm{~B})$ & 94.9 \\
\hline $\mathrm{H}(30 \mathrm{~A})-\mathrm{C}(30)-\mathrm{H}(30 \mathrm{~B})$ & 103.2 \\
\hline $\mathrm{N}(26)-\mathrm{C}(30)-\mathrm{H}(30 \mathrm{E})$ & 97.7 \\
\hline$C(35)-C(30)-H(30 E)$ & 97.7 \\
\hline $\mathrm{N}(26)-\mathrm{C}(30)-\mathrm{H}(30 \mathrm{~F})$ & 97.7 \\
\hline $\mathrm{C}(35)-\mathrm{C}(30)-\mathrm{H}(30 \mathrm{~F})$ & 97.7 \\
\hline $\mathrm{H}(30 \mathrm{E})-\mathrm{C}(30)-\mathrm{H}(30 \mathrm{~F})$ & 103.7 \\
\hline $\mathrm{N}(26)-\mathrm{C}(30)-\mathrm{H}(30 \mathrm{C})$ & 98.2 \\
\hline $\mathrm{C}(31)-\mathrm{C}(30)-\mathrm{H}(30 \mathrm{C})$ & 98.2 \\
\hline$N(26)-C(30)-H(30 D)$ & 98.2 \\
\hline$C(31)-C(30)-H(30 D)$ & 98.2 \\
\hline$H(30 C)-C(30)-H(30 D)$ & 103.7 \\
\hline
\end{tabular}




$\begin{array}{lc}\mathrm{C}(32)-\mathrm{C}(31)-\mathrm{C}(30) & 140(9) \\ \mathrm{C}(32)-\mathrm{C}(31)-\mathrm{H}(31) & 110.0 \\ \mathrm{C}(30)-\mathrm{C}(31)-\mathrm{H}(31) & 110.0 \\ \mathrm{C}(31)-\mathrm{C}(32)-\mathrm{H}(32 \mathrm{~A}) & 120.0 \\ \mathrm{C}(31)-\mathrm{C}(32)-\mathrm{H}(32 \mathrm{~B}) & 120.0 \\ \mathrm{H}(32 \mathrm{~A})-\mathrm{C}(32)-\mathrm{H}(32 \mathrm{~B}) & 120.0 \\ \mathrm{C}(34)-\mathrm{C}(33)-\mathrm{C}(30) & 136(6) \\ \mathrm{C}(34)-\mathrm{C}(33)-\mathrm{H}(33) & 112.1 \\ \mathrm{C}(30)-\mathrm{C}(33)-\mathrm{H}(33) & 112.1 \\ \mathrm{C}(33)-\mathrm{C}(34)-\mathrm{H}(34 \mathrm{~A}) & 120.0 \\ \mathrm{C}(33)-\mathrm{C}(34)-\mathrm{H}(34 \mathrm{~B}) & 120.0 \\ \mathrm{H}(34 \mathrm{~A})-\mathrm{C}(34)-\mathrm{H}(34 \mathrm{~B}) & 120.0 \\ \mathrm{C}(36)-\mathrm{C}(35)-\mathrm{C}(30) & 162(10) \\ \mathrm{C}(36)-\mathrm{C}(35)-\mathrm{H}(35) & 99.2 \\ \mathrm{C}(30)-\mathrm{C}(35)-\mathrm{H}(35) & 99.2 \\ \mathrm{C}(35)-\mathrm{C}(36)-\mathrm{H}(36 \mathrm{~A}) & 120.0 \\ \mathrm{C}(35)-\mathrm{C}(36)-\mathrm{H}(36 \mathrm{~B}) & 120.0 \\ \mathrm{H}(36 \mathrm{~A})-\mathrm{C}(36)-\mathrm{H}(36 \mathrm{~B}) & 120.0\end{array}$

Symmetry transformations used to generate equivalent atoms:

Table S23. Anisotropic displacement parameters $\left(\AA^{2} \times 10^{3}\right)$ for 17-A. The anisotropic displacement factor exponent takes the form: $-2 p^{2}\left[h^{2} a^{* 2} U^{11}+\ldots+2 h k a^{*} b^{*} U^{12}\right]$

\begin{tabular}{lcccccc}
\hline & $U^{11}$ & $U^{22}$ & $U^{33}$ & $U^{23}$ & $U^{13}$ & $U^{12}$ \\
\hline $\mathrm{O}(1)$ & $59(3)$ & $28(3)$ & $72(4)$ & $3(3)$ & $3(3)$ & $-7(2)$ \\
$\mathrm{O}(2)$ & $48(3)$ & $32(3)$ & $68(4)$ & $2(2)$ & $11(3)$ & $-1(2)$ \\
$\mathrm{O}(3)$ & $49(3)$ & $40(3)$ & $79(4)$ & $13(3)$ & $21(3)$ & $2(2)$ \\
$\mathrm{O}(4)$ & $68(4)$ & $65(4)$ & $83(5)$ & $9(4)$ & $-7(4)$ & $12(3)$ \\
$\mathrm{O}(5)$ & $59(3)$ & $36(3)$ & $64(4)$ & $3(3)$ & $11(3)$ & $4(2)$ \\
$\mathrm{O}(6)$ & $51(3)$ & $39(3)$ & $81(4)$ & $-8(3)$ & $9(3)$ & $-3(3)$ \\
$\mathrm{O}(7)$ & $62(3)$ & $16(2)$ & $73(4)$ & $-3(2)$ & $-9(3)$ & $5(2)$ \\
$\mathrm{O}(8)$ & $52(3)$ & $23(2)$ & $68(4)$ & $-4(2)$ & $-3(3)$ & $-1(2)$ \\
$\mathrm{O}(9)$ & $61(3)$ & $28(3)$ & $92(4)$ & $-14(3)$ & $0(3)$ & $1(3)$ \\
& & & & 98 & &
\end{tabular}




$\begin{array}{lcccccc}\mathrm{N}(1) & 48(4) & 40(4) & 64(5) & -5(3) & 4(3) & 3(3) \\ \mathrm{N}(2) & 87(6) & 26(3) & 83(6) & -11(3) & -21(5) & 5(3) \\ \mathrm{N}(26) & 92(6) & 80(6) & 78(6) & 24(5) & 4(5) & 9(5) \\ \mathrm{C}(1) & 48(4) & 21(3) & 72(5) & 4(3) & 8(4) & -3(3) \\ \mathrm{C}(2) & 41(4) & 29(4) & 73(6) & 3(4) & 8(4) & 4(3) \\ \mathrm{C}(3) & 42(4) & 29(4) & 61(5) & 8(3) & 9(3) & 0(3) \\ \mathrm{C}(4) & 48(4) & 27(4) & 61(5) & 8(3) & 5(4) & 6(3) \\ \mathrm{C}(5) & 51(5) & 29(4) & 74(6) & 8(4) & 8(4) & 7(3) \\ \mathrm{C}(6) & 62(5) & 30(4) & 59(5) & 4(4) & 14(4) & 3(4) \\ \mathrm{C}(7) & 59(5) & 25(3) & 63(5) & 9(4) & 17(4) & 6(3) \\ \mathrm{C}(8) & 64(5) & 39(4) & 70(6) & 1(4) & 16(4) & 3(4) \\ \mathrm{C}(9) & 58(5) & 25(4) & 65(6) & -1(3) & 8(4) & -7(3) \\ \mathrm{C}(10) & 74(6) & 45(5) & 63(6) & 16(4) & 4(4) & -2(4) \\ \mathrm{C}(11) & 67(5) & 30(4) & 84(7) & 1(4) & 6(5) & -6(4) \\ \mathrm{C}(12) & 52(4) & 35(4) & 69(6) & -7(4) & 12(4) & 0(4) \\ \mathrm{C}(13) & 46(4) & 24(4) & 74(6) & 2(3) & 10(4) & -1(3) \\ \mathrm{C}(14) & 46(4) & 28(4) & 61(5) & -3(3) & 3(4) & -3(3) \\ \mathrm{C}(15) & 37(4) & 23(3) & 73(5) & -4(4) & 8(4) & -2(3) \\ \mathrm{C}(16) & 51(4) & 32(4) & 66(5) & -4(4) & 11(4) & -3(3) \\ \mathrm{C}(17) & 58(5) & 35(4) & 60(5) & 3(4) & 9(4) & -7(3) \\ \mathrm{C}(18) & 57(5) & 45(5) & 65(6) & -4(4) & 14(4) & -9(4) \\ \mathrm{C}(19) & 58(5) & 34(4) & 64(5) & 1(4) & 11(4) & -6(4) \\ \mathrm{C}(20) & 50(4) & 15(3) & 67(5) & -2(3) & -4(4) & -2(3) \\ \mathrm{C}(21) & 52(4) & 17(3) & 62(5) & -1(3) & -3(4) & 0(3) \\ \mathrm{C}(22) & 60(5) & 37(4) & 85(7) & 10(4) & 13(5) & 12(4) \\ \mathrm{C}(23) & 64(5) & 33(4) & 83(7) & 10(4) & 12(5) & -11(4) \\ \mathrm{C}(24) & 68(6) & 44(5) & 88(7) & 12(5) & 25(5) & 2(4) \\ \mathrm{C}(25) & 69(6) & 40(5) & 92(7) & 7(4) & 28(5) & 9(4) \\ \mathrm{C}(27) & 54(5) & 71(6) & 82(6) & -15(6) & 13(5) & -11(5) \\ \mathrm{C}(28) & 72(6) & 27(4) & 88(7) & -15(4) & -10(5) & -9(4) \\ \mathrm{C}(29) & 52(5) & 34(4) & 72(6) & -16(4) & 3(4) & -8(4) \\ \mathrm{C}(30) & 219(17) & 184(19) & 197(18) & 6(17) & 87(13) & 36(15) \\ \mathrm{C}(31) & 206(19) & 206(19) & 205(19) & 0(3) & 54(6) & 0(3) \\ \mathrm{C}(32) & 116(15) & 115(16) & 114(15) & -1(6) & 28(7) & 1(6) \\ \mathrm{C}(33) & 190(20) & 150(40) & 60(20) & 20(30) & 40(18) & 40(20) \\ \mathrm{C}(34) & 210(30) & 210(30) & 210(30) & 0(6) & 55(10) & 1(6) \\ & & & & & & \end{array}$




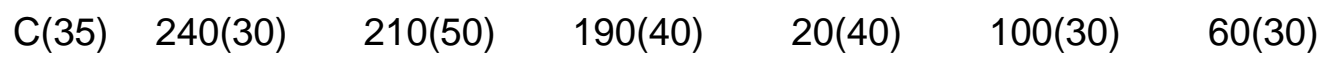

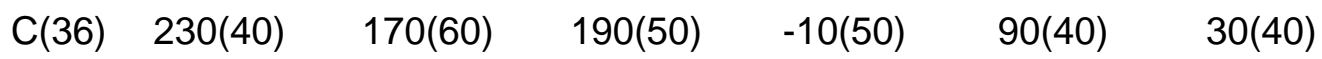

Table S24. Hydrogen coordinates ( x 104) and isotropic displacement parameters $\left(\AA^{2} \times 10^{3}\right)$ for 17-A.

\begin{tabular}{|c|c|c|c|c|}
\hline & $x$ & $y$ & $z$ & $\mathrm{U}(\mathrm{eq})$ \\
\hline $\mathrm{H}(2)$ & 6928 & 6159 & 5110 & 75 \\
\hline $\mathrm{H}(1)$ & 5773 & 2145 & 6023 & 63 \\
\hline $\mathrm{H}(2 \mathrm{~A})$ & 10398 & 6883 & 10824 & 88 \\
\hline $\mathrm{H}(2 \mathrm{~B})$ & 10352 & 5078 & 10455 & 88 \\
\hline $\mathrm{H}(26)$ & 4882 & 2899 & 1808 & 104 \\
\hline $\mathrm{H}(2 \mathrm{C})$ & 7921 & 7339 & 6975 & 59 \\
\hline $\mathrm{H}(3)$ & 9393 & 6838 & 6177 & 54 \\
\hline $\mathrm{H}(4)$ & 8473 & 4586 & 5674 & 57 \\
\hline $\mathrm{H}(5)$ & 7971 & 7077 & 4316 & 63 \\
\hline $\mathrm{H}(6 \mathrm{~A})$ & 7758 & 4857 & 3276 & 61 \\
\hline $\mathrm{H}(6 \mathrm{~B})$ & 7020 & 4834 & 3839 & 61 \\
\hline $\mathrm{H}(7)$ & 7947 & 2575 & 4738 & 59 \\
\hline $\mathrm{H}(8 \mathrm{~A})$ & 7058 & 1584 & 2959 & 70 \\
\hline $\mathrm{H}(8 \mathrm{~B})$ & 7215 & 416 & 3826 & 70 \\
\hline $\mathrm{H}(12)$ & 3869 & 3193 & 4232 & 63 \\
\hline $\mathrm{H}(17)$ & 6134 & 3643 & 7036 & 63 \\
\hline $\mathrm{H}(18)$ & 5894 & 3667 & 8797 & 68 \\
\hline $\mathrm{H}(19)$ & 7361 & 4170 & 9315 & 64 \\
\hline $\mathrm{H}(20)$ & 7476 & 4200 & 7516 & 57 \\
\hline $\mathrm{H}(21)$ & 8127 & 6612 & 8458 & 56 \\
\hline$H(22 A)$ & 10033 & 5110 & 7209 & 92 \\
\hline $\mathrm{H}(22 \mathrm{~B})$ & 9473 & 3576 & 7488 & 92 \\
\hline$H(22 C)$ & 10197 & 4607 & 8238 & 92 \\
\hline$H(23 A)$ & 7895 & 9130 & 5546 & 92 \\
\hline $\mathrm{H}(23 \mathrm{~B})$ & 8797 & 9043 & 5202 & 92 \\
\hline
\end{tabular}




\begin{tabular}{|c|c|c|c|c|}
\hline$H(23 C)$ & 8846 & 9737 & 6183 & 92 \\
\hline$H(24 A)$ & 9331 & 5880 & 3318 & 98 \\
\hline $\mathrm{H}(24 \mathrm{~B})$ & 9999 & 7185 & 3963 & 98 \\
\hline $\mathrm{H}(24 \mathrm{C})$ & 8949 & 7654 & 3573 & 98 \\
\hline$H(25 A)$ & 8714 & 2460 & 3246 & 98 \\
\hline$H(25 B)$ & 8823 & 952 & 3959 & 98 \\
\hline$H(25 C)$ & 9257 & 2779 & 4263 & 98 \\
\hline $\mathrm{H}(27 \mathrm{~A})$ & 3741 & 3481 & 7405 & 105 \\
\hline $\mathrm{H}(27 \mathrm{~B})$ & 4540 & 2956 & 8255 & 105 \\
\hline $\mathrm{H}(27 \mathrm{C})$ & 4051 & 1535 & 7556 & 105 \\
\hline $\mathrm{H}(28 \mathrm{~A})$ & 7668 & 1334 & 7151 & 102 \\
\hline $\mathrm{H}(28 \mathrm{~B})$ & 7294 & 926 & 8002 & 102 \\
\hline$H(28 C)$ & 8227 & 96 & 7917 & 102 \\
\hline$H(30 A)$ & 5865 & 3968 & 1279 & 232 \\
\hline $\mathrm{H}(30 \mathrm{~B})$ & 5894 & 1999 & 1215 & 232 \\
\hline$H(30 E)$ & 6551 & 3780 & 2011 & 232 \\
\hline $\mathrm{H}(30 \mathrm{~F})$ & 5783 & 3663 & 1128 & 232 \\
\hline$H(30 C)$ & 5722 & 2956 & 1016 & 232 \\
\hline$H(30 D)$ & 6370 & 1769 & 1699 & 232 \\
\hline$H(31)$ & 6860 & 4951 & 1195 & 247 \\
\hline$H(32 A)$ & 8075 & 2317 & 1513 & 138 \\
\hline $\mathrm{H}(32 \mathrm{~B})$ & 8204 & 4255 & 1164 & 138 \\
\hline $\mathrm{H}(33)$ & 7221 & 2017 & 1120 & 158 \\
\hline$H(34 A)$ & 7658 & 5584 & 1503 & 256 \\
\hline$H(34 B)$ & 8265 & 4218 & 1097 & 256 \\
\hline $\mathrm{H}(35)$ & 6476 & 2261 & 530 & 245 \\
\hline$H(36 A)$ & 8082 & 1458 & 1465 & 226 \\
\hline $\mathrm{H}(36 \mathrm{~B})$ & 7584 & 1385 & 390 & 226 \\
\hline
\end{tabular}

Table S25. Torsion angles [०] for 17-A

\begin{tabular}{lr}
\hline$C(2)-O(1)-C(1)-C(22)$ & $112.1(9)$ \\
$C(2)-O(1)-C(1)-C(21)$ & $-107.4(7)$ \\
$C(1)-O(1)-C(2)-C(3)$ & $-118.1(8)$
\end{tabular}




$\begin{array}{lc}\text { O(1)-C(1)-C(2)-C(3) } & 104.1(8) \\ \mathrm{C}(22)-\mathrm{C}(1)-\mathrm{C}(2)-\mathrm{C}(3) & 0.1(12) \\ \mathrm{C}(21)-\mathrm{C}(1)-\mathrm{C}(2)-\mathrm{C}(3) & -155.7(7) \\ \mathrm{C}(22)-\mathrm{C}(1)-\mathrm{C}(2)-\mathrm{O}(1) & -103.9(8) \\ \mathrm{C}(21)-\mathrm{C}(1)-\mathrm{C}(2)-\mathrm{O}(1) & 100.3(7) \\ \mathrm{O}(1)-\mathrm{C}(2)-\mathrm{C}(3)-\mathrm{C}(4) & 156.2(6) \\ \mathrm{C}(1)-\mathrm{C}(2)-\mathrm{C}(3)-\mathrm{C}(4) & 85.1(9) \\ \mathrm{O}(1)-\mathrm{C}(2)-\mathrm{C}(3)-\mathrm{C}(23) & -77.9(8) \\ \mathrm{C}(1)-\mathrm{C}(2)-\mathrm{C}(3)-\mathrm{C}(23) & -149.0(7) \\ \mathrm{C}(2)-\mathrm{C}(3)-\mathrm{C}(4)-\mathrm{O}(2) & 43.8(8) \\ \mathrm{C}(23)-\mathrm{C}(3)-\mathrm{C}(4)-\mathrm{O}(2) & -79.5(8) \\ \mathrm{C}(2)-\mathrm{C}(3)-\mathrm{C}(4)-\mathrm{C}(5) & 169.9(6) \\ \mathrm{C}(23)-\mathrm{C}(3)-\mathrm{C}(4)-\mathrm{C}(5) & 46.6(9) \\ \mathrm{C}(24)-\mathrm{O}(3)-\mathrm{C}(5)-\mathrm{C}(4) & -158.8(7) \\ \mathrm{C}(24)-\mathrm{O}(3)-\mathrm{C}(5)-\mathrm{C}(6) & 74.7(9) \\ \mathrm{O}(2)-\mathrm{C}(4)-\mathrm{C}(5)-\mathrm{O}(3) & -179.2(6) \\ \mathrm{C}(3)-\mathrm{C}(4)-\mathrm{C}(5)-\mathrm{O}(3) & 55.3(8) \\ \mathrm{O}(2)-\mathrm{C}(4)-\mathrm{C}(5)-\mathrm{C}(6) & -52.7(8) \\ \mathrm{C}(3)-\mathrm{C}(4)-\mathrm{C}(5)-\mathrm{C}(6) & -178.2(6) \\ \mathrm{O}(3)-\mathrm{C}(5)-\mathrm{C}(6)-\mathrm{C}(7) & 66.2(10) \\ \mathrm{C}(4)-\mathrm{C}(5)-\mathrm{C}(6)-\mathrm{C}(7) & -55.6(10) \\ \mathrm{C}(5)-\mathrm{C}(6)-\mathrm{C}(7)-\mathrm{C}(25) & -75.4(10) \\ \mathrm{C}(5)-\mathrm{C}(6)-\mathrm{C}(7)-\mathrm{C}(8) & 160.1(8) \\ \mathrm{C}(25)-\mathrm{C}(7)-\mathrm{C}(8)-\mathrm{C}(9) & -175.5(7) \\ \mathrm{C}(6)-\mathrm{C}(7)-\mathrm{C}(8)-\mathrm{C}(9) & -170.1(8) \\ \mathrm{C}(7)-\mathrm{C}(8)-\mathrm{C}(9)-\mathrm{C}(10) & -50.5(10) \\ \mathrm{C}(7)-\mathrm{C}(8)-\mathrm{C}(9)-\mathrm{C}(14) & 114.2(10) \\ \mathrm{C}(30)-\mathrm{N}(26)-\mathrm{C}(10)-\mathrm{C}(9) & -65.0(10) \\ \mathrm{C}(30)-\mathrm{N}(26)-\mathrm{C}(10)-\mathrm{C}(11) & -15(3) \\ \mathrm{C}(14)-\mathrm{C}(9)-\mathrm{C}(10)-\mathrm{N}(26) & 167(2) \\ \mathrm{C}(8)-\mathrm{C}(9)-\mathrm{C}(10)-\mathrm{N}(26) & 178.7(9) \\ \mathrm{C}(14)-\mathrm{C}(9)-\mathrm{C}(10)-\mathrm{C}(11) & -0.5(14) \\ \mathrm{C}(8)-\mathrm{C}(9)-\mathrm{C}(10)-\mathrm{C}(11) & -3.9(12) \\ \mathrm{N}(26)-\mathrm{C}(10)-\mathrm{C}(11)-\mathrm{O}(4) & 176.9(8) \\ \mathrm{C}(9)-\mathrm{C}(10)-\mathrm{C}(11)-\mathrm{O}(4) & 7.6(12) \\ \mathrm{N}(26)-\mathrm{C}(10)-\mathrm{C}(11)-\mathrm{C}(12) & \\ & \end{array}$


$\mathrm{C}(9)-\mathrm{C}(10)-\mathrm{C}(11)-\mathrm{C}(12)$

$\mathrm{O}(4)-\mathrm{C}(11)-\mathrm{C}(12)-\mathrm{C}(13)$

$\mathrm{C}(10)-\mathrm{C}(11)-\mathrm{C}(12)-\mathrm{C}(13)$

$\mathrm{C}(11)-\mathrm{C}(12)-\mathrm{C}(13)-\mathrm{N}(1)$

$\mathrm{C}(11)-\mathrm{C}(12)-\mathrm{C}(13)-\mathrm{C}(14)$

$\mathrm{C}(15)-\mathrm{N}(1)-\mathrm{C}(13)-\mathrm{C}(12)$

$\mathrm{C}(15)-\mathrm{N}(1)-\mathrm{C}(13)-\mathrm{C}(14)$

$\mathrm{C}(10)-\mathrm{C}(9)-\mathrm{C}(14)-\mathrm{O}(5)$

$\mathrm{C}(8)-\mathrm{C}(9)-\mathrm{C}(14)-\mathrm{O}(5)$

$C(10)-C(9)-C(14)-C(13)$

$\mathrm{C}(8)-\mathrm{C}(9)-\mathrm{C}(14)-\mathrm{C}(13)$

$\mathrm{C}(12)-\mathrm{C}(13)-\mathrm{C}(14)-\mathrm{O}(5)$

$\mathrm{N}(1)-\mathrm{C}(13)-\mathrm{C}(14)-\mathrm{O}(5)$

$\mathrm{C}(12)-\mathrm{C}(13)-\mathrm{C}(14)-\mathrm{C}(9)$

$\mathrm{N}(1)-\mathrm{C}(13)-\mathrm{C}(14)-\mathrm{C}(9)$

$\mathrm{C}(13)-\mathrm{N}(1)-\mathrm{C}(15)-\mathrm{O}(6)$

$\mathrm{C}(13)-\mathrm{N}(1)-\mathrm{C}(15)-\mathrm{C}(16)$

$\mathrm{O}(6)-\mathrm{C}(15)-\mathrm{C}(16)-\mathrm{C}(17)$

$\mathrm{N}(1)-\mathrm{C}(15)-\mathrm{C}(16)-\mathrm{C}(17)$

$\mathrm{O}(6)-\mathrm{C}(15)-\mathrm{C}(16)-\mathrm{C}(27)$

$\mathrm{N}(1)-\mathrm{C}(15)-\mathrm{C}(16)-\mathrm{C}(27)$

$\mathrm{C}(27)-\mathrm{C}(16)-\mathrm{C}(17)-\mathrm{C}(18)$

$\mathrm{C}(15)-\mathrm{C}(16)-\mathrm{C}(17)-\mathrm{C}(18)$

$\mathrm{C}(16)-\mathrm{C}(17)-\mathrm{C}(18)-\mathrm{C}(19)$

$\mathrm{C}(17)-\mathrm{C}(18)-\mathrm{C}(19)-\mathrm{C}(20)$

$\mathrm{C}(28)-\mathrm{O}(7)-\mathrm{C}(20)-\mathrm{C}(19)$

$\mathrm{C}(28)-\mathrm{O}(7)-\mathrm{C}(20)-\mathrm{C}(21)$

$\mathrm{C}(18)-\mathrm{C}(19)-\mathrm{C}(20)-\mathrm{O}(7)$

$\mathrm{C}(18)-\mathrm{C}(19)-\mathrm{C}(20)-\mathrm{C}(21)$

$\mathrm{C}(29)-\mathrm{O}(8)-\mathrm{C}(21)-\mathrm{C}(1)$

$\mathrm{C}(29)-\mathrm{O}(8)-\mathrm{C}(21)-\mathrm{C}(20)$

$\mathrm{O}(1)-\mathrm{C}(1)-\mathrm{C}(21)-\mathrm{O}(8)$

$\mathrm{C}(22)-\mathrm{C}(1)-\mathrm{C}(21)-\mathrm{O}(8)$

$\mathrm{C}(2)-\mathrm{C}(1)-\mathrm{C}(21)-\mathrm{O}(8)$

$\mathrm{O}(1)-\mathrm{C}(1)-\mathrm{C}(21)-\mathrm{C}(20)$

$C(22)-C(1)-C(21)-C(20)$
8.2(12)

174.5(8)

$-3.7(12)$

178.1(7)

$-4.3(11)$

0.9(13)

$-176.8(7)$

179.0(8)

$-1.7(10)$

$-4.1(11)$

175.2(6)

$-174.2(7)$

3.8(9)

8.7(10)

$-173.4(7)$

14.4(12)

$-166.5(7)$

$-157.1(8)$

23.7(10)

21.2(11)

$-157.9(8)$

$3.0(14)$

$-178.9(8)$

172.4(9)

$-1.4(14)$

73.0(10)

$-161.7(8)$

$-102.7(9)$

135.5(8)

87.5(8)

$-147.3(7)$

$-89.4(7)$

49.4(9)

$-154.5(6)$

150.3(6)

$-70.9(9)$ 
$\mathrm{C}(2)-\mathrm{C}(1)-\mathrm{C}(21)-\mathrm{C}(20)$

$\mathrm{O}(7)-\mathrm{C}(20)-\mathrm{C}(21)-\mathrm{O}(8)$

$\mathrm{C}(19)-\mathrm{C}(20)-\mathrm{C}(21)-\mathrm{O}(8)$

$\mathrm{O}(7)-\mathrm{C}(20)-\mathrm{C}(21)-\mathrm{C}(1)$

$\mathrm{C}(19)-\mathrm{C}(20)-\mathrm{C}(21)-\mathrm{C}(1)$

$\mathrm{C}(21)-\mathrm{O}(8)-\mathrm{C}(29)-\mathrm{O}(9)$

$\mathrm{C}(21)-\mathrm{O}(8)-\mathrm{C}(29)-\mathrm{N}(2)$

$\mathrm{C}(10)-\mathrm{N}(26)-\mathrm{C}(30)-\mathrm{C}(33)$

$\mathrm{C}(10)-\mathrm{N}(26)-\mathrm{C}(30)-\mathrm{C}(35)$

$\mathrm{C}(10)-\mathrm{N}(26)-\mathrm{C}(30)-\mathrm{C}(31)$

$\mathrm{N}(26)-\mathrm{C}(30)-\mathrm{C}(31)-\mathrm{C}(32)$

$\mathrm{N}(26)-\mathrm{C}(30)-\mathrm{C}(33)-\mathrm{C}(34)$

$\mathrm{N}(26)-\mathrm{C}(30)-\mathrm{C}(35)-\mathrm{C}(36)$
85.1(8)

$-52.6(9)$

70.8(8)

70.1(8)

$-166.5(7)$

20.7(13)

$-158.4(8)$

$-5(14)$

$80(11)$

$-59(11)$

$119(13)$

$-79(15)$

$-81(37)$

Symmetry transformations used to generate equivalent atoms:

Table S26. Hydrogen bonds for 17-A [ $\AA$ and $\left.{ }^{\circ}\right]$.

\begin{tabular}{lcccc}
\hline $\mathrm{D}-\mathrm{H} \ldots \mathrm{A}$ & $\mathrm{d}(\mathrm{D}-\mathrm{H})$ & $\mathrm{d}(\mathrm{H} \ldots \mathrm{A})$ & $\mathrm{d}(\mathrm{D} \ldots \mathrm{A})$ & $<(\mathrm{DHA})$ \\
\hline $\mathrm{O}(2)-\mathrm{H}(2) \ldots \mathrm{O}(6) \# 1$ & 0.84 & 2.01 & $2.832(8)$ & 167.0 \\
$\mathrm{~N}(2)-\mathrm{H}(2 \mathrm{~A}) \ldots \mathrm{O}(7) \# 2$ & 0.88 & 2.15 & $2.909(9)$ & 143.7 \\
$\mathrm{~N}(2)-\mathrm{H}(2 \mathrm{~B}) \ldots \mathrm{O}(9) \# 3$ & 0.88 & 2.03 & $2.894(10)$ & 166.8 \\
\hline
\end{tabular}

Symmetry transformations used to generate equivalent atoms:

$\# 1-x+1, y+1 / 2,-z+1 \quad \# 2-x+2, y+1 / 2,-z+2 \quad \# 3-x+2, y-1 / 2,-z+2$ 


\section{References}

1. Romney, D. K.; Colvin, S. M.; Miller, S. J., Catalyst Control over Regio- and Enantioselectivity in Baeyer-Villiger Oxidations of Functionalized Ketones. J. Am. Chem. Soc. 2014, 136, 14019-14022.

2. Lichtor, P. A.; Miller, S. J., Combinatorial evolution of site- and enantioselective catalysts for polyene epoxidation. Nat Chem 2012, 4, 990-995.

3. Hsieh, S.-Y.; Tang, Y. U.; Crotti, S.; Stone, E.; Miller, S. J., Catalytic Enantioselective Pyridine N-Oxidation. J. Am. Chem. Soc. 2019, 141, 18624-18629.

4. Storch, G.; Kim, B.; Mercado, B. Q.; Miller, S. J., A Stereodynamic Redox-Interconversion Network of Vicinal Tertiary and Quaternary Carbon Stereocenters in Hydroquinone-Quinone Hybrid Dihydrobenzofurans. Angew. Chem. Int. Ed. 2018, 57, 15107-15111.

5. Nawrat, C. C.; Lewis, W.; Moody, C. J., Synthesis of Amino-1,4-benzoquinones and Their Use in Diels-Alder Approaches to the Aminonaphthoquinone Antibiotics. J. Org. Chem. 2011, 76, 7872-7881.

6. CrysAlisPro, R. O., The Woodlands, TX, 2015.

7. Sheldrick, G. M., A Short History of SHELX. Acta Crystallogr A. 2008, A64, 112-122.

8. Spek, A. L., PLATON SQUEEZE: a tool for the calculation of the disordered solvent contribution to the calculated structure factors. Acta Cryst 2015, (C71), 9-18. 
